\title{
Marketing, Morality, and the Natural Environment
}

\author{
Andrew Crane
}

Thesis submitted to the University of Nottingham for the degree of Doctor of Philosophy, October, 1998.

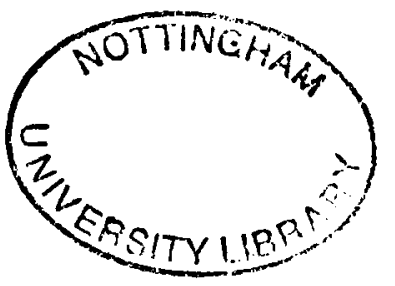




\section{CONTENTS}

ACKNOWLEDGEMENTS

i

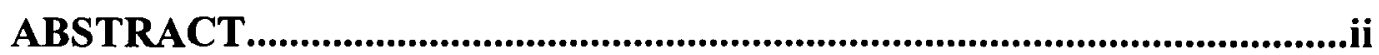

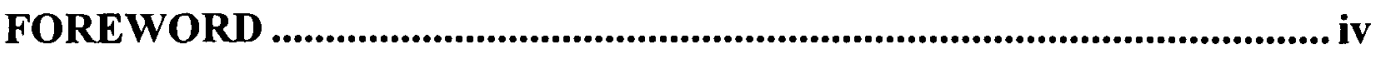

\section{MARKETING, MORALITY AND THE NATURAL}

ENVIRONMENT: AN INTRODUCTION .................................................... 1

1.1 INTRODUCTION .......................................................................... 1

1.2 AIMS AND CONTRIBUTION OF THE THESIS ............................................... 3

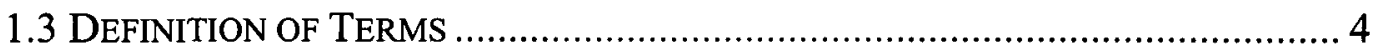

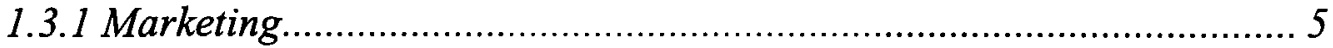

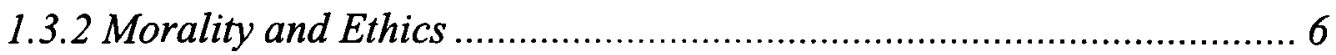

1.3.3 Natural Environment ................................................................ 9

1.3.4 Organizational Culture................................................................. 10

1.3.5 Corporate Social Responsibility ............................................... 12

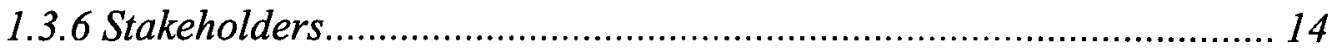

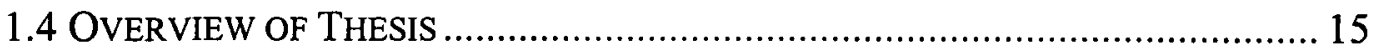

2. MARKETING AND MORALITY: PERSPECTIVES, ISSUES,

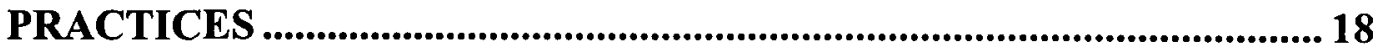

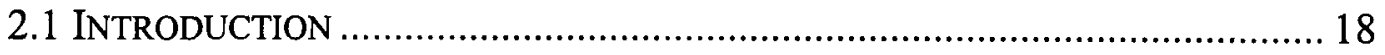

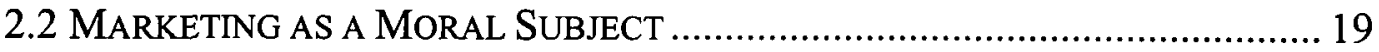

2.2.1 The Moral Basis of Marketing..................................................... 20

2.2.2 The Functioning of the Marketing System .................................... 21

2.2.3 Impact of Marketing on Society .................................................... 25

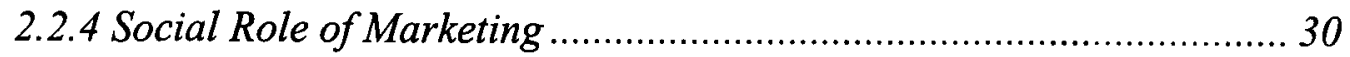

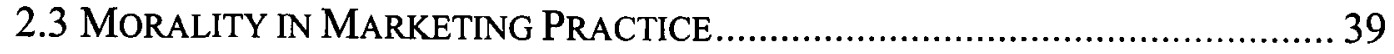

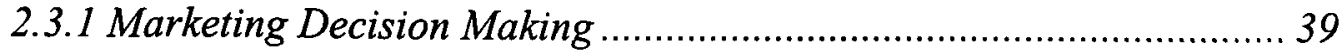

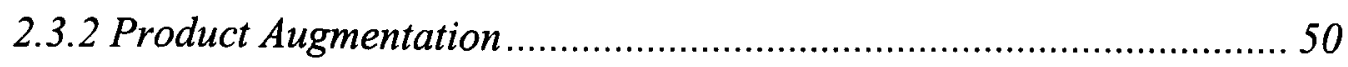


2.4 Towards a Research AgEnda: EXPloring Moral MEANING IN

MARKETING

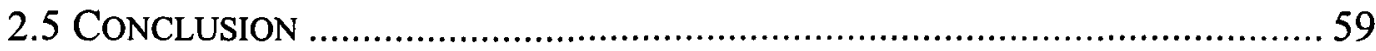

3. GREEN MARKETING: A MORAL VIEW ....................................... 62

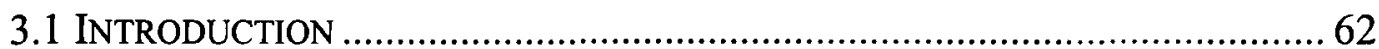

3.2 THE GREEN MARKETING LitERATURE ................................................ 64

3.3 GreEn MARKETING AS A Moral DomaIN ..........................................6 68

3.4 Moral PERSPECTIVES ON GreEn MARKeting ....................................... 71

3.4.1 'Shallow Green' Perspectives.................................................. 71

3.4.2 'Deep Green' Perspectives ...................................................... 79

3.5 Morality in Green Marketing: An Organization Culture

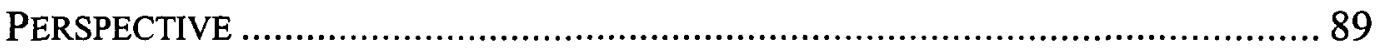

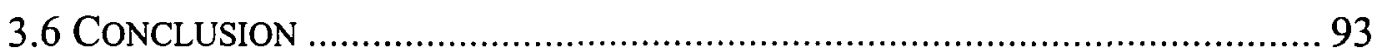

\section{TRUTH, KNOWLEDGE AND METHOD IN RESEARCHING}

MORALITY IN MARKETING............................................................... 95

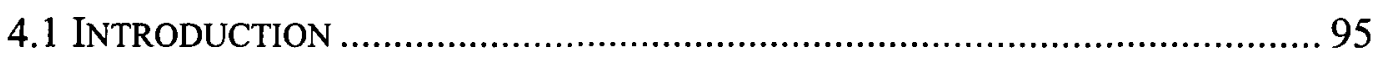

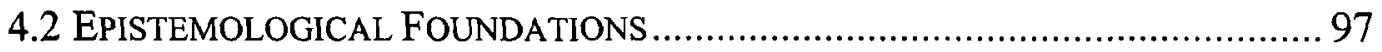

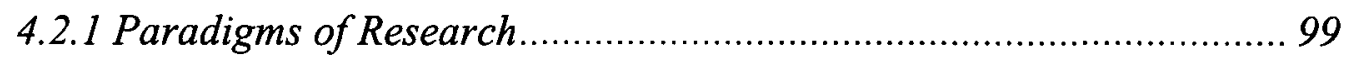

4.3 Researching Morality in MaRKeting - Methodological

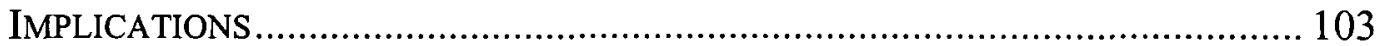

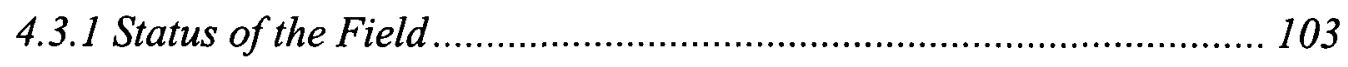

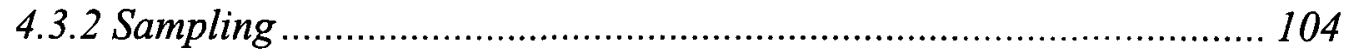

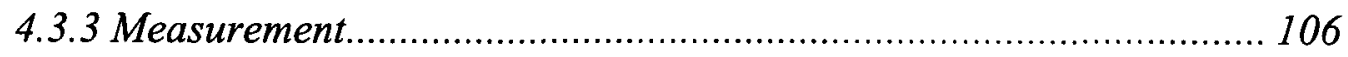

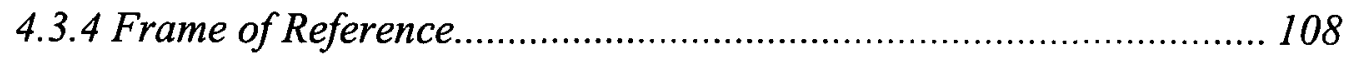

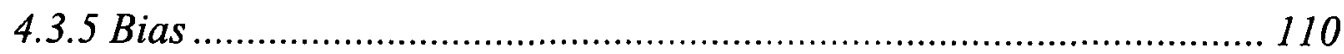

4.3.6 Context ............................................................................ 111

4.4 RESEARCHING ORGANIZATIONS AS CULTURES .................................... 113

4.4.1 Symbolic-Cultural Analysis ................................................... 114

4.4.2 Political-Cultural Analysis ...................................................... 116

4.5 Research Strategy \& Method: A Grounded TheOry APPROACH... 117 
4.5.1 The Case Study Research Strategy ........................................ 118

4.5.2 Data Collection........................................................................ 123

4.5.3 Data Analysis and Theory Development .................................... 129

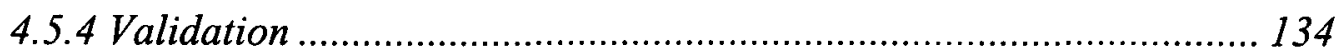

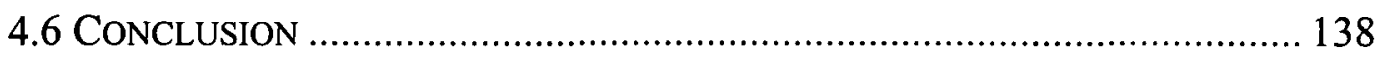

5. CONVENTIONAL BUSINESS ORGANIZATIONS ......................... 140

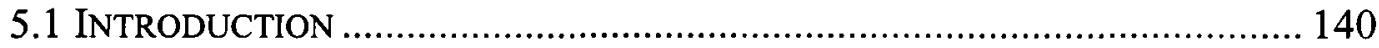

5.2 BACKGROUND TO THE CASE STUDIES .................................................. 141

5.2.1 Boots the Chemist (BTC) ....................................................... 142

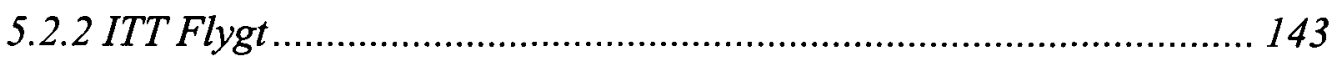

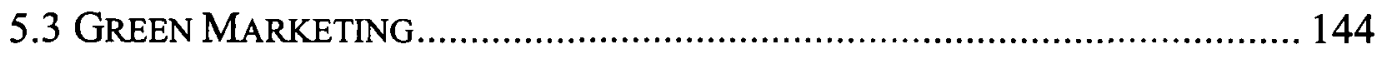

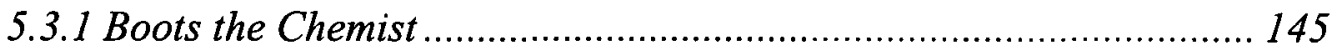

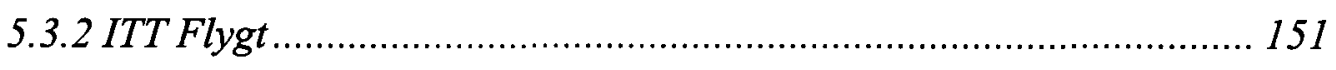

5.3.3 Green Marketing and Corporate Social Responsibility ................. 154

5.4 CUltural DYNAMiCS OF GrEen MARKETING .................................... 155

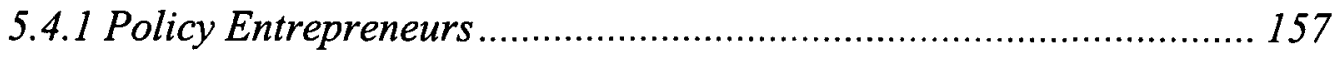

5.4.2 Processes of Institutionalisation................................................ 160

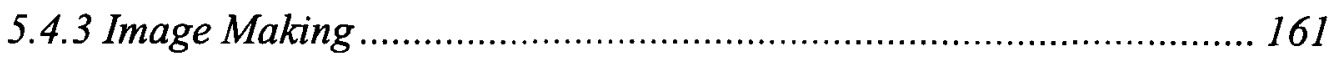

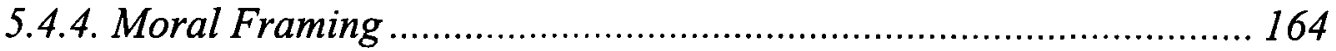

5.4.5 Narrative Surfing............................................................... 170

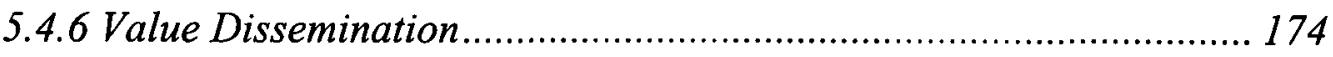

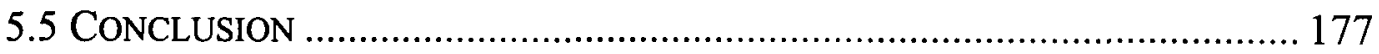

6. SOCIAL MISSION COMPANIES ............................................... 180

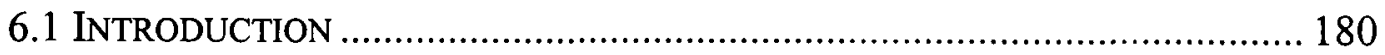

6.2 BACKGROUND TO THE CASE STUdIES................................................ 182

6.2.1 Co-operative Wholesale Society (CWS) ........................................ 182

6.2.2 Ecology Building Society (the Ecology) ................................... 185

6.2.3 Beauty Without Cruelty (BWC) ............................................. 187

6.2.4 Bioregional Charcoal Company (Bioregional) ............................. 190

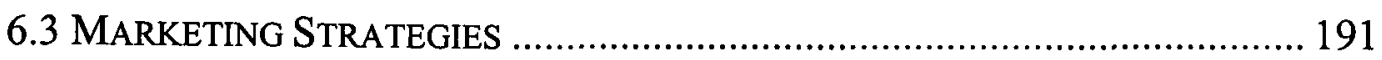




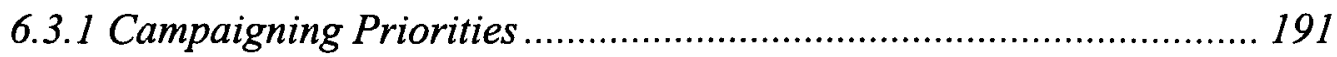

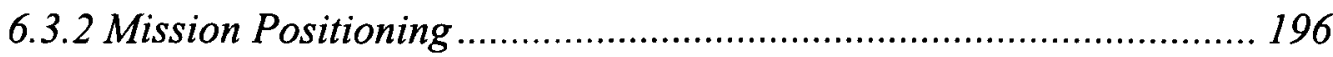

6.3.3 Strategies for Social Mission Marketing ........................................ 200

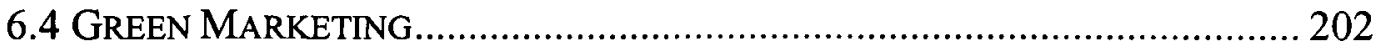

6.4.1 Green Marketing and Social Responsibility ................................... 207

6.5 CUlTURAL DYNAMICS OF GREEN MARKETING ….................................. 210

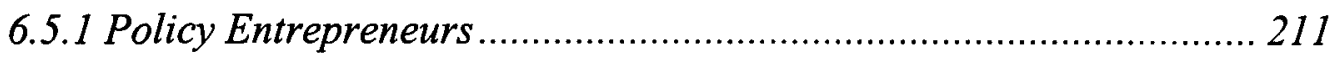

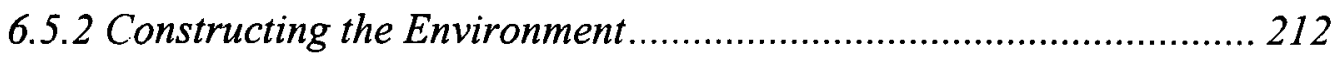

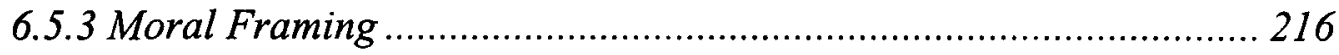

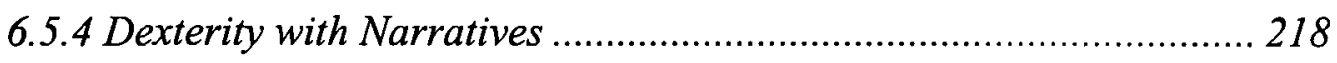

6.5.5 Disseminating Environmental Values............................................... 221

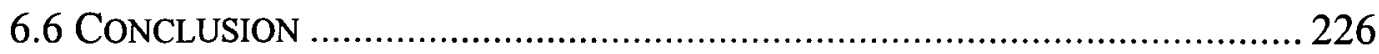

7. BUSINESS-NGO COLLABORATION .................................................. 230

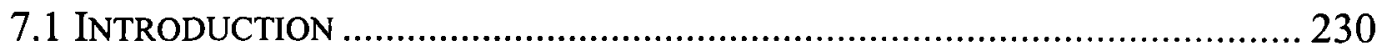

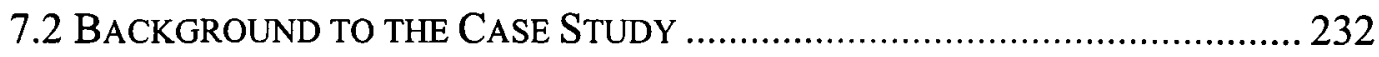

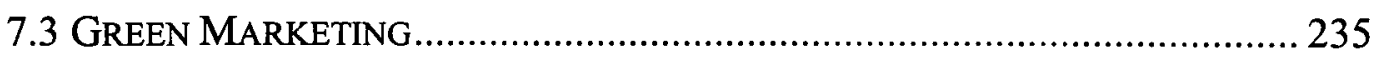

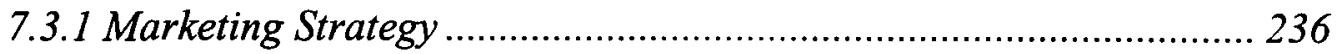

7.3.2 Industrial Networks and Supply Chain Management ...................... 237

7.3.3 Marketing Relationships ................................................................ 240

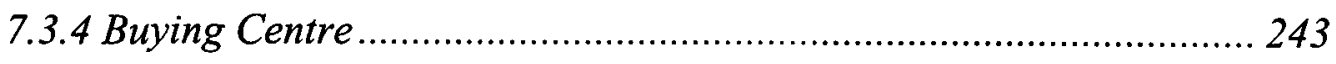

7.3.5 Green Marketing and Social Responsibility .................................... 244

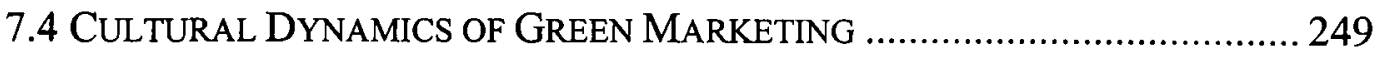

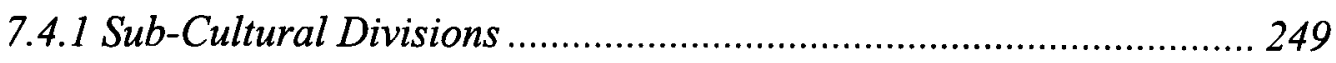

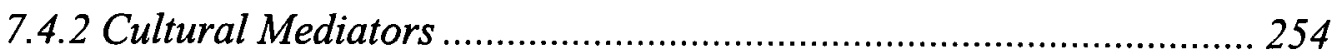

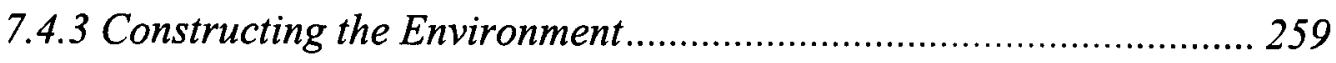

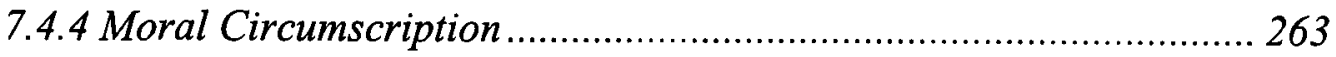

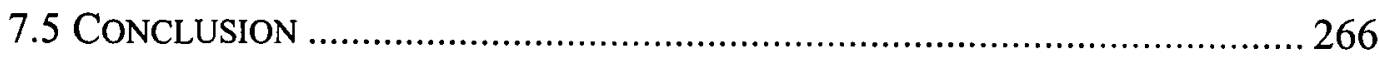

8. GREEN MARKETING AND MORALITY - EVIDENCE FROM

THREE APPROACHES.......................................................................... 270

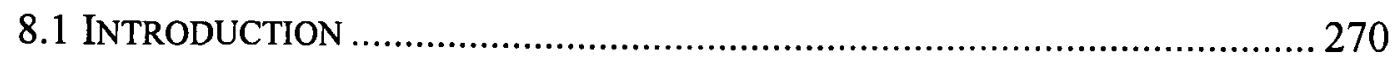


8.2 GREEN MARKETING IN PRACTICE .................................................... 271

8.2.1 Strategic Responses ............................................................... 275

8.2.2 Marketing Mix Policies........................................................... 278

8.2.3 Green Marketing Theory ..................................................... 288

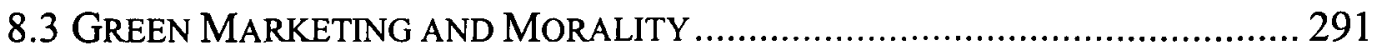

8.3.1 Green Marketing as a Moral Subject .......................................... 291

8.3.2 Morality in Green Marketing Practice ....................................... 299

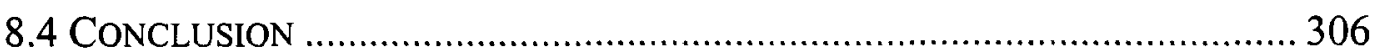

9. AMORALIZATION, MORALIZATION, AND THE MANAGEMENT OF MORALITY IN MARKETING ........................................................ 310

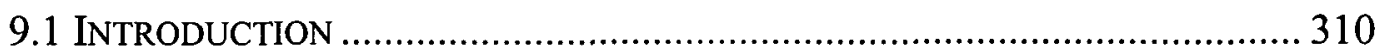

9.2 AMORALIZATION IN GREEN MARKETING.............................................. 311

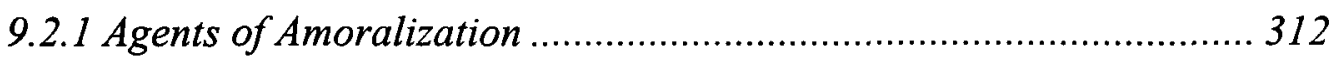

9.2.2 Processes of Amoralization ...................................................... 315

9.2.3 Causes of Amoralization.............................................................. 324

9.2.4 Consequences of Amoralization ................................................ 332

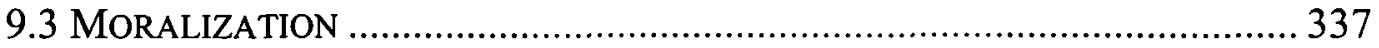

9.3.1 Moralization Through Culture Change ...................................... 337

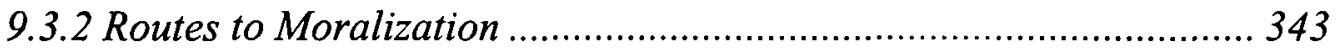

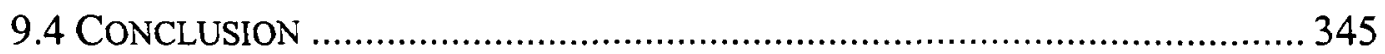

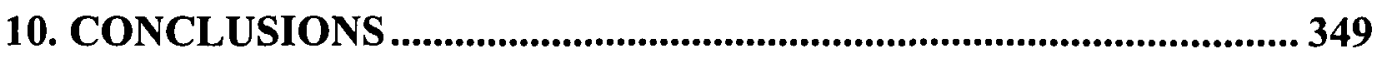

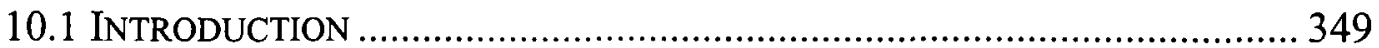

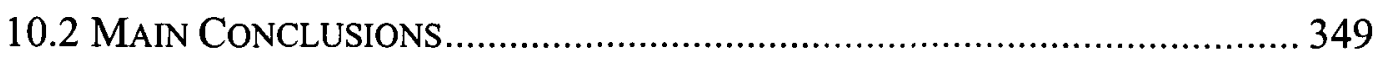

10.2.1 Marketing as a Moral Domain ................................................... 350

10.2.2 Green Marketing Theory and Practice....................................... 351

10.2.3 Cultural Dynamics of Green Marketing .................................. 352

10.2.4 Moral Meaning in Marketing .................................................. 353

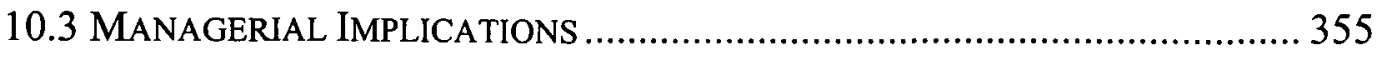

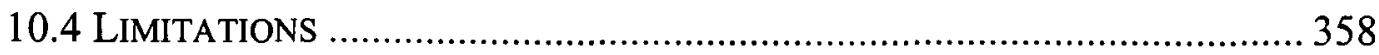

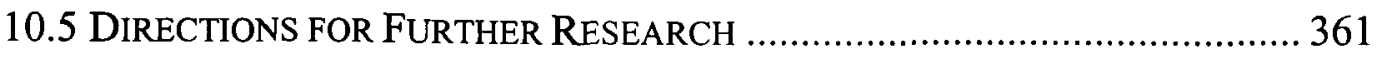


REFERENCES 365

APPENDIX A: SAMPLE CONTACT LETTERS AND RESEARCH PROPOSALS 392

APPENDIX B: 1995 PLUS GROUP MEMBERS .404 


\section{Acknowledgements}

I would like to thank my supervisors, Professors Christine Ennew and Ken Starkey for the help, ideas, insight and encouragement that they have given me during the preparation of this thesis. My thanks also to Mick Rowlinson for his invaluable support and advice.

This work would also not have been possible without the time and information provided by the various respondents whose reflections and opinions illuminate the text, and bring to life the practical realities of marketing, morality and the natural environment. My sincere thanks then to the following organizations: B\&Q, BBC Magazines, Beauty Without Cruelty, Bioregional Charcoal, Boots the Chemist, CWS, Do It All, Ecology Building Society, EC Waltons, ITT Flygt, Laing Homes, Mainstream Environmental Consultants, Railtrack, Spicers, Strategic Environmental Consulting, WH Smith, Wickes, WWF UK.

Finally, I should like to express my gratitude to the University of Nottingham Business School, and more latterly Cardiff Business School, for the time, facilities and financial support which have enabled me to undertake and complete the doctoral studies which have resulted in the production of this thesis. 


\section{Abstract}

This thesis is a study of morality in marketing, focusing specifically on marketing in relation to the natural environment, i.e. green marketing. An interdisciplinary approach is adopted, incorporating a broad range of literature pertaining to issues of morality in marketing. The contribution of this literature is analysed, and the green marketing literature is assessed according to the moral perspectives applied. The need for greater insight into the development and communication of moral meanings in the marketing process is thus identified, and an organization culture perspective is articulated by which to effect this in the context of green marketing. This cultural perspective is then used as the basis for the exploratory, empirical research which forms the core of the thesis.

Utilising an interpretive, comparative case study approach, and following a grounded theory methodology, data is presented to illustrate the content and process of green marketing strategies and tactics in three types of organizations - conventional, social mission, and business-NGO collaboration. The moral tone of these projects is examined and the cultural dynamics which attend the greening process are explored in order to reveal the moral meanings subsequently communicated and legitimised within and around the case organizations.

Consequently, a picture of contemporary green marketing practice grounded in empirical data is developed, and the theoretical implications discussed. Moreover, the proposition is advanced that there is a tendency in corporations for green marketing to be accompanied by a process of 'amoralization', i.e. a removal of moral meaning and significance for the natural environment. Specific actor roles and processes of amoralization are set out, and potential explanations advanced. The implications and wider ramifications of these 
findings are discussed, and the possibilities for introducing greater moral consciousness and feeling into marketing are discussed. Finally, some management implications and directions for further research are considered. 


\section{Foreword}

Some of the work in this thesis, in a significantly revised form, has appeared in publications elsewhere.

Material from chapter 4 (mainly 4.2 and 4.3 ) is to be published as: Crane, A. (1998), "Are You Ethical? Please Tick Yes or No : On Researching Ethics in Business Organizations", Journal of Business Ethics, in press.

Chapter 5 has been published in revised form as: Crane, A. (1997), "The Dynamics of Marketing Ethical Products: A Cultural Perspective", Journal of Marketing Management, vol. 13 (6), pp. 561-577.

Chapter 7 has been published in a revised form as: Crane, A. (1998), “Exploring Green Alliances”, Journal of Marketing Management, vol. 14 (6), pp. 559-580.

Some of the material in chapter 9 (section 9.3.1) was adapted from: Crane, A. (1995), "Rhetoric and Reality in the Greening of Organizational Culture", Greener Management International, vol. 12, pp. 49-62. 


\section{Marketing, Morality and the Natural Environment: An Introduction}

\subsection{Introduction}

This thesis is a study of morality in marketing. It is a fascinating, complex and important area of investigation, and the particular subject upon which the main focus of enquiry is directed - the natural environment - is perhaps one of the most significant and controversial aspects of the contemporary debate surrounding this area. However, despite being an area much commented on in various academic and non-academic texts, it is at present one that has been subjected to relatively little in-depth scholarly enquiry. This represents both advantages and disadvantages for the current study. It is an advantage in the sense that it means there is still much of interest to the aspiring researcher which requires thorough investigation. It is a disadvantage in that conceptual development in this area is relatively poor, and therefore a greater breadth of literature must be consulted in order to build a solid base for theory development.

The approach adopted here then is very much an interdisciplinary one. Obviously by identifying an area of study such as "marketing, morality and the natural environment" the academic range is almost by definition beyond any single literature. It is necessary certainly to span the three literatures of marketing, business ethics and green business which are home to the main subjects under investigation. Critically however, this is also very much a study of organizations, of their management, and of the individuals that people them. Therefore, by the very nature of the engagement with the research area, this 
thesis is also strongly related to the organization studies, organizational behaviour, and management studies literatures. In a broader sense, it is even influenced and informed to some extent by a whole range of texts depicting issues of morality and/or the environment in business and marketing, from fields as diverse as philosophy (e.g. Baudrillard, 1981), economics (e.g. Galbraith, 1977), politics (e.g. Gorz, 1980), cultural studies (e.g. Williamson, 1978), even literature (e.g. Unsworth, 1992) and film (e.g. Wall Street).

Given such a broad scope, there is clearly some sense in maintaining a narrow focus of investigation. It is mainly for this reason that the decision was taken to concentrate attention on one particular area of morality in marketing, i.e. that pertaining to the natural environment. Certainly, the environment is an important and interesting case to follow for it brings up quite specific and pronounced moral issues. Also it is currently a high profile business issue, and is regarded by many as a critical social problem. Moreover, given various criticisms that have been forwarded concerning the superficiality of the existing green business literature and associated empirical work (Gladwin, 1993; Newton and Harte, 1997), there is clearly a case for the application of more in-depth and sophisticated research such as is presented in this thesis. Further guidance on how the substantive area of the environment fits into the formal field of marketing and morality is provided, where relevant, during the course of the thesis.

The main aim of this introductory chapter is to give a general overview of the thesis. Hence, the succeeding sections are as follows: in section 1.2, the aims and principal contributions of the thesis are set out in order to explain what exactly has been achieved (and why this is important); the next section, 1.3, deals with the definition of terms used, such as to provide the reader with the 
means to understand the terminology employed; and finally, section 1.4 provides an overview of the thesis structure and content in order to aid in successful navigation through the document.

\subsection{Aims and Contribution of the Thesis}

The first core aim of the thesis is to investigate the role and significance of morality in the practice of marketing. This is approached as a largely empirical question, and the response forwarded is based primarily on analysis of the opinions, reflections and sensibilities of organization members involved in marketing, rather than on the researcher's own views. It is then in large measure a descriptive investigation into morality rather then a normative or prescriptive one. Therefore, one of the principal contributions of the thesis is its provision of new and unique insights into the moral dimension of marketing practice and practitioners in the 1990s.

A second core aim, instrumental to the first, is to determine how exactly it is that notions of morality are given or denied expression and meaning within the marketing process. By focusing specifically on marketing involved in some way with the natural environment, the concern is thus with how organization members experience and communicate the moral dimension of these 'green' marketing activities. This is an important research question for it has implications for how marketing theory should be conceptualised, and for determining the appropriate role for morality within this conceptualisation. Hence the thesis makes a contribution to marketing theory as well as developing an important new approach to the study of business ethics, i.e. the study of moral meaning. 
In order to achieve this, it is necessary of course to examine in considerable detail the relevant 'green' marketing behaviours which are to be assessed according to their moral dimensions. Given, as shall be shown in chapter 3, a relatively unimpressive extant literature in this respect, a third core aim of the thesis is therefore to provide a detailed study of these behaviours and in particular of the role of morality within them. A further contribution is provided then in terms of strengthening the theoretically and empirically 'thin' green marketing literature.

The fourth core aim of the thesis is to provide a more compelling organizational culture perspective on business and marketing ethics and on organizational greening than is currently in evidence. The intention in this respect is to bring the knowledge accrued in the organization studies literature to bear more substantially on these other literatures. Combined with this, it is the aim of this thesis to answer some of the strong methodological criticisms that have been levelled at the business ethics and green business literatures (see chapter 4), and in this way also contribute to the development of more rigorous theory in these areas.

These can be regarded as the main aims of the study. Having then set these out, attention is turned now to defining some of the key terms and concepts used during the thesis.

\subsection{Definition of Terms}

During the course of this account, a wide range of terms and concepts have been employed, many of which might be regarded as open to different interpretations. The purpose of this section then is to clarify the particular 
meanings applied in this thesis, and to justify why it has been deemed appropriate to use them in these particular ways. To begin, the terms used in the thesis title are defined - 'marketing', 'morality', and 'natural environment'; following this, three other terms which are used throughout the thesis and which might be regarded as conceptually ambiguous are defined 'organizational culture', 'social responsibility', and 'stakeholders'.

\subsubsection{Marketing}

The term "marketing" is used in its broadest sense here to describe any of a number of related activities associated with the common end of facilitating and securing exchanges between an organization and consumers. To use a distinction more often used in the strategy literature, marketing is viewed here as comprising of both content (what marketing decisions are made and which marketing activities are undertaken) as well as process (how those decisions are reached, and how those activities come to take place). Indeed, in the light of the work of leading strategy writers such as Pettigrew (e.g. 1973, 1979, 1985) and Mintzberg (e.g. 1989, 1990, 1991), and the exhortations of critical marketing theorists, such as Brownlie et al. (1994), much of the focus in this study is on the process of marketing, such as to discover how marketing is conducted rather than how it should be conducted. Hence, the principal unit of analysis is not the marketing department as such, nor indeed specialist marketing personnel, but rather the marketing process, i.e. the sequence of events that gives rise to specific marketing phenomena, and ultimately, to exchange behaviour. Given a focus on the natural environment, many of the relevant events and decisions that were analysed during fieldwork occurred outside of the marketing department; however, as long they were observed to have had some bearing on the marketing process, they were still regarded as 
marketing or marketing-related phenomena.

\subsubsection{Morality and Ethics}

For the purpose of this study, morality and ethics are assumed to be different but related constructs. At the most basic level, morality is used to refer to any distinction between right and wrong, good and evil, either in thought, feeling or behaviour; ethics is used to refer to a moral code, or system of rules, through which judgements of right and wrong etc. can be made. This separation arose initially from the need to conceptualise a form of morality which was appropriate to a descriptive form of ethics rather than a prescriptive one. This meant that it was necessary to employ a linguistic distinction between the moral experience of organizational members (how they think and feel about morality; how notions of morality impinge upon behaviour; how a sense of morality is recognised and expressed, etc.) and the means by which those thoughts, feelings and behaviours can be judged. Parker (1997:3), in a sharp criticism of the business ethics project, makes a strong case for the possibility of such a distinction:

Another way to justify this suspension of judgement about judgement is (what philosophers sometimes call) 'descriptive' as opposed to 'prescriptive' 'Ethics'. This is also sometimes framed as morals - mores, norms, values - as opposed to ethics - codes, rules, tablets from the mountain .... I suppose this is to 'sociologise' the 'Ethical', to draw it down from its supposed lofty place into the flow of the ordinary... It means refusing to accept a division of labour that distinguishes the 'Ethical' from the other things that people do in particular times and places .... So if we accept this social construction of the 'Ethical' .... then this effectively presses upon us a suspension of our 
judgement, an attempt to go beyond good and evil, a gesture at relativism in the interests of a thicker description.

So 'morality' and 'moral' can be regarded as largely descriptive labels, 'ethics' and 'ethical' as prescriptive or judgmental ones. The main focus in this study, as it shall become clear, is the former; nonetheless 'ethics', in their formal sense, have not been dispensed with, and the principal ethical theories, the grand narratives of deontology, teleology, and virtue ethics have been used to provide important insight into marketing moralities. As such, a short introduction to these, as well as the increasingly prominent ethical relativist position, is justified before continuing with the definition of terms ${ }^{1}$.

\section{Deontology}

Deontology is essentially an ethic of duty. Actions are deemed to be right or wrong according to the motivations of the person performing them, rather than according to the likely or actual consequences of the action. Kant is generally regarded as having provided the definitive exposition of deontological ethics (Chryssides and Kaler, 1993). His conclusions are based on two concepts: that human reason is the only basis for establishing morality; and that moral reasoning should be based on the 'categorical imperative', i.e. one ought never to act unless one is willing to have the maxim on which one acts become a universal law (Robin and Reidenbach, 1987).

\footnotetext{
${ }^{1}$ More in depth discussion of ethical theories as they relate to business can be found in most mainstream business ethics textbooks. See for example, Beauchamp and Bowie (1988); De George (1990); Hoffman and Moore (1990); Chryssides and Kaler (1993). For a discussion in relation to marketing specifically, see Robin and Reidenbach (1987, 1993); Laczniak and Murphy (1993).
} 


\section{Teleology}

Otherwise known as consequentialist ethics, teleological ethics is in many ways the opposite of deontology. Activities are deemed to be right or wrong depending on the outcomes of the action, not the type or form of the actions themselves. Utilitarianism is probably the best known consequentialist theory of ethics, and its formulation owes mainly to the work of Bentham and Mill. At a basic level, utilitarianism can be summed up by the phrase "the greatest good for the greatest number". This might be assessed according to some form of cost/benefit analysis which identifies aggregate social happiness as a result of particular activities. Utilitarianism is generally regarded to provide the ethical rationale for market capitalism since economic theory is predicated on the principle of providing the greatest material good for the greatest number (Robin and Reidenbach, 1987; Cannon, 1992).

\section{Virtue Ethics}

Whilst less developed in the context of business ethics than deontological and teleological theories, virtue ethics is, at the time of writing, receiving renewed interest and support (Laczniak and Murphy, 1993; Collier, 1995). Though its original formulation is generally attributed to Aristotle, much of the contemporary interest in, and prominence given, to virtue ethics is on account of the work of MacIntyre (1984). He defines virtue as acquired human qualities, or dispositions, that enable persons to achieve 'the good' in their chosen vocations. Hence the focus in virtue ethics is on the 'goodness' of the actor rather than the action. 


\section{Ethical Relativism}

Whilst there is certainly a tradition of ethical relativism, it has become popularised by, and increasingly associated with, postmodernist perspectives on ethics. These centre on a denial of the possibility of ethical grand narratives which can be applied to any given activity, and focus instead on moral customs that are time, culture and context dependent. Hence, what is right at a particular time, in a given culture, in a particular set of circumstances, may be wrong at another time, or in a different culture, or in another context. More radical positions deny even the possibility of any commonality of ethical principles, suggesting an extreme moral relativism (Robin and Reidenbach, 1987).

\subsubsection{Natural Environment}

Definitions of what constitutes the 'natural', the 'environmental', 'ecology', 'greening', and related concepts are highly complex and continue to occupy scholars in environmental ethics, politics, sociology, organization studies, and various other areas of enquiry (e.g. Gorz, 1980; Naess, 1989; Gladwin, 1993; Shrivastava, 1994; Macnaghten and Urry, 1995). In this study, the definition employed for the natural environment has remained deliberately non-specific, such as to more effectively elicit the meanings applied by organization members. This is important from a moral perspective since different meanings reflect different ethical positions and assumptions (Naess, 1989; Shrivastava, 1993). Accordingly, the range of terms relating to the natural environment are used fairly indiscriminately, unless a more precise definition is necessary to develop a particular point, or to reflect the meaning intended by an interviewee or author. 
In the context of marketing, the environment is usually considered within the rubric of either green/environmental marketing or green consumerism, reflecting a focus on the organization or consumption respectively. This study, as an organizational analysis, predominantly uses the former with green marketing preferred, purely on stylistic grounds, over environmental marketing. However, the meaning applied to green marketing here is not as in many other treatments: it refers simply to marketing phenomena where some account has been taken of environmental concerns; it should not be understood to imply that these phenomena are deemed to be necessarily good (or bad) for the environment. Hence, the use of a product label claiming "environmentally friendly", a reduction in product packaging, and the introduction of clean technology in the product production process would all be regarded as examples of green marketing. The explanation for this usage, and its implications for the study overall, are discussed in more detail in chapter 3.

\subsubsection{Organizational Culture}

The concept of organizational culture is again one that is not uniformly understood and applied in existing literature. Indeed, one of the leading organization culture theorists, Joanne Martin (1992) refers to the "state of conceptual chaos" that afflicts the academic study of organization culture. Basically, culture is regarded here as being concerned with a broad range of phenomena: various artefacts such as behaviours, stories, myths, symbols, language, etc.; cognitive beliefs, values, attitudes, and codes; and basic, takenfor-granted assumptions (Schein, 1992). These are the basic contents of the culture concept. Whilst the detail of the actual approach to determining what should or should not be regarded as being, or being part of, the organization culture is set out in chapters 2 and 3, three central conceptual distinctions are 
worthy of brief attention here. Smircich (1983a) sets out two basic research agendas underlying the cultural analysis of organizations: culture as a critical variable (i.e. culture is viewed as a managerial tool with which to manipulate performance), and culture as a root metaphor (i.e. it is a way of understanding the organization). Whilst the first might be regarded as the approach taken by researchers whom Martin (1985) refers to as the "cultural pragmatists", i.e. those who see culture in terms of how it can be managed, the second corresponds to the approach of those she refers to as the "cultural purists", i.e. those who are concerned more with the sociological and anthropological study of organizations. The present account should be regarded as being situated largely in the latter category. This agenda adopts the idea of culture as an epistemological device with which to frame the study of the social terrain of the organization (Smircich, 1983a) - the implications of which are considered in chapter 4 .

The advantage of this perspective is that it allows for a full appreciation of the range of other possible conceptualisations of culture without having to identify any category as the single correct one. Martin's (1992) distinction between three perspectives on culture - integration, differentiation, and fragmentation for example is an important one, and is utilised during this study. The integration perspective assumes that there is a single unitary culture which is widely and relatively unambiguously shared throughout the organization. The differentiation perspective identifies unity mainly at the subcultural level and focuses on the conflict and inconsistency experienced between these. The fragmentation perspective assumes little cultural consensus and concentrates attention on the ambiguity and complexity of the cultural terrain. In this study, all of these are considered valid to the extent that they help to understand the multiplicity of ways that the term 'culture' is used by researchers and 
organization members. As far as possible, it is indicated where a particular meaning is intended. Likewise, Brown (1995a) distinguishes between espoused culture, i.e. the desired state of the organization and the culture that is presented to external publics, and culture-in-practice, i.e. the culture experienced by organization members. Whilst the main concern here is the latter, the former is regarded as an important cultural artefact in itself.

\subsubsection{Corporate Social Responsibility}

The definition of 'corporate social responsibility' (abbreviated here to 'social responsibility') is again much disputed, and can be used to describe very different perspectives and phenomena (Sethi, 1974; Stroup and Neubert, 1987; Clarkson, 1995; Smith, 1990). Moreover, 'social responsibility' and 'business ethics' have often been used interchangeably in existing literature but, although related, are conceptually different in certain critical respects (Epstein, 1987; Robin and Reidenbach, 1987). Epstein's (1987) analysis is useful in setting out the distinction. He argues that social responsibility focuses on the products of business activity and its various impacts on society. As such, it is based on the expectations and claims that are put upon businesses in terms of their impact on, or involvement in, society, beyond that which is prescribed by law ${ }^{2}$. Basic questions of social responsibility thus consider the role of businesses in causing and solving social problems, and in promoting general social welfare. In contrast, business ethics, he argues, concentrates on the moral refection and choices that individuals within organizations are faced

\footnotetext{
${ }^{2}$ Whilst it is most common for social responsibility to be regarded as existing beyond legal requirement, not all conceptualisations share this perspective. Carroll (1979) for instance categorises social responsibility in terms of economic, legal, ethical and discretionary components. Hence we are concerned here only with the latter two of his components.
} 
with; essentially it is concerned with questions of right and wrong. Clearly then, all questions of social responsibility could probably be addressed from a business ethics perspective ${ }^{3}$, although other perspectives (e.g. economics, sociology, etc.) would also be valid. Only some questions of business ethics however are likely to be relevant to social responsibility concerns. For example, it could be argued that the taking of bribes by employees is unlikely to have any significant bearing on the role of the corporation in society.

Notwithstanding these distinctions, there is nonetheless a marked tendency in much of the existing social responsibility literature for the term social responsibility to be used in a purely positive or approving sense, i.e. in normative terms of being (or not being) socially responsible. However, this is only one dimension of a concept which is as much a perspective on the relationship between business and society as it a judgement of the social performance of particular organizations. Thus, the concept of social responsibility here is used in its broader sense, i.e. as a way of looking at the role of business organizations in society, or a particular lens through which to reflect and structure social reality ${ }^{4}$.

\footnotetext{
${ }^{3}$ Indeed, business ethics texts usually contain substantial chapters on social responsibility, and the rights and wrongs of different arguments in this respect (again see Beauchamp and Bowie, 1988; De George, 1990; Hoffman and Moore, 1990; Chryssides and Kaler, 1993).

4 The concept of social responsibility has spawned a couple of off-shoots, social responsiveness and social performance, which have their roots, respectively, in the mode or strategy of response of the corporation to social issues, and in the social outcomes of corporate activity. These concepts are not central to this study, but for an in-depth discussion of how they relate to social responsibility, see Wood (1991) and Swanson (1995).
} 


\subsubsection{Stakeholders}

Stakeholder theory proposes that the firm exists at the centre of a series of contractual resource-dependant relationships with multiple interested groups within society (Freeman, 1984). Stakeholders are therefore those parties both inside and outside the organization which in some way affect, and are affected by, its behaviour. Whilst the set of constituencies that constitute the firm's stakeholder group is open to some debate (Donaldson and Preston, 1995), this study uses Frederick et al.'s (1992) model. This posits that the firm has two sets of stakeholders: primary (here referred to as "economic") stakeholders shareholders, employees, resellers, customers, competitors, suppliers and creditors - with which it interacts through the market in the context of some form of economic exchange; and secondary (here "non-economic") stakeholders - local communities, regulators, pressure groups, the media, the general public and business support groups - which it interacts with outside of the market ${ }^{5}$.

According to Donaldson and Preston (1995), this essentially descriptive theory that the firm can be conceptualised as a network of stakeholder relationships, has been used as a basis for two further articulations of stakeholder theory: a normative argument which proposes that firms should be managed according to their duty to provide for the benefit of these stakeholders (Evan and Freeman, 1988); and an instrumental argument that stakeholder strategies provide for improved performance, either ethically (Freeman and Gilbert, 1988), socially (Frederick et al., 1992), or in terms of more traditional strategic

\footnotetext{
5 The labels "economic" and "non-economic" stakeholders are used in preference to "primary" and "secondary" in order to avoid any suggestion of precedence of the former over the latter. Frederick et al. (1992:11) confirm that such a suggestion is not their intention.
} 
and economic goals (Johnson and Scholes, 1997). In the current study, it is the descriptive form of stakeholder theory that is principally used; where uses other than this are made, it is indicated in the text.

\subsection{Overview of Thesis}

Having set out the main aims of the study, and provided a detailed clarification of the terminology that is used throughout, the final task of this introductory chapter is to explain the structure of the thesis, and provide guidance on the content of the subsequent chapters.

In the next chapter, chapter 2 , the basic foundations of the area of marketing and morality are examined. Taking a structured and interdisciplinary approach, the essential elements of the broad literature considered to impinge upon this area are reviewed. Following this review, it is argued that the area of moral meaning in marketing at present remains largely unexamined, and the basic framework for the current study is considered.

In chapter 3 , the focus of the study is narrowed to a single set of marketing behaviours, i.e. 'green marketing'. The green marketing literature is examined in the context of marketing and morality, and categorised according to the 'deep' versus 'shallow' green distinction. Since this literature is found to be largely wanting of theoretical development, a potentially more fruitful approach is set out. It is here that the concept of organizational culture is introduced and three general research questions are articulated.

The philosophical perspective of the study, and research method employed during the empirical section, are described and justified in chapter 4 . It is 
shown how a interpretive mode of engagement might offer substantial epistemological potential over the functionalist paradigm in the area of study under examination. It is explained why the comparative case study approach was chosen to conduct the enquiry, and the three organizational approaches to green marketing which have been investigated are introduced. These are: conventional organizations (those with economic goals clearly prominent), social mission organizations (those with social goals clearly prominent), and business-NGO collaboration (those involving both profit-making and not-forprofit organizations). The grounded theory mode of qualitative research which has been adopted in this study is explained and the mechanics of its application described. Details are thus given of the sampling procedures, data collected, and methods of analysis and theory development.

Chapters 5-7 deal with the main results of the empirical work. Each chapter relates to a different organizational approach, with conventional organizations, social mission companies, and business-NGO alliances being the subjects of chapters 5,6 , and 7 respectively. Whilst the format of these chapters differs somewhat in order to explore the particular issues arising during data analysis, a basic framework is used to maintain some degree of consistency. Therefore, during all of these chapters some basic background details of the case organizations are given, their general approaches to green marketing explained, and the relationship of green marketing with notions of social responsibility examined. The largest part of each chapter however is given over to describing the cultural dynamics of green marketing observed in the case organizations, and analysing the implications for moral meaning and for morality generally.

In the final section of the thesis, chapters $8-10$, these results are discussed, 
their implications examined, and conclusions reached. Chapter 8 deals specifically with green marketing as observed in the case organizations. In the first half of the chapter, the results from chapters 5-7 are compared, contrasted and integrated, such that an extensive, empirically-based examination of contemporary green marketing practice is presented. This is then used as a basis for exploring possible theoretical development. In the second half of the chapter, the moral dimension of green marketing as observed in the case organizations is set out, utilising the concepts and categories introduced in chapters 2 and 3.

In chapter 9, the discussion is extended to examine in detail some of the principal findings of the thesis, namely the phenomena of amoralization and moralization. These are described in some depth and their possible antecedents and consequences explored. This discussion is then used as a basis to explore the likely implications for the management of morality in marketing.

Finally, in chapter 10 the thesis is concluded. This includes a review of the main findings and implications of the study, a discussion of managerial implications, examination of possible limitations, and suggestions for future research. The thesis ends with a brief summary of its main contributions. 


\section{Marketing and Morality: Perspectives, Issues, Practices}

\subsection{Introduction}

Marketing is an extraordinarily rich field in which to study morality. Because the marketing function provides an interface between the organization and its environment, marketing activities are highly visible to the general public and many of the most renowned, even infamous, examples of corporate ethical abuse over the years have concerned the activities of the marketing department (Murphy and Laczniak, 1981; Laczniak and Murphy, 1993). The use and content of advertising in particular has been the subject of considerable moral debate from both within and outside of the marketing subject area (Greyser, 1972; Pollay, 1986; Phillips, 1997). This is not to say though that the only moral interest in marketing is of a negative type. Product buyers have been shown to take into consideration the ethics, integrity, or trustworthiness of sellers in making their purchase decisions (Smith, 1990); and equally sellers have sought to capitalise on their moral stances by using them to assist in achieving marketing objectives (Owen and Scherer, 1993).

There is then by now a large and fairly impressive literature concerned with morality in marketing, often coming under the banner of marketing ethics, a subfield of business ethics (Laczniak and Murphy, 1993). However, in presenting a review of the literature surrounding marketing and morality, it is necessary to reach beyond the perimeters of the marketing ethics literature alone and consider also work from a number of other fields and sub-fields such as business ethics, business and society, marketing, economics, industrial sociology, organizational 
then, marketing has traditionally been ethically justified from a consequentialist position - i.e. by facilitating the satisfaction of consumer needs, it maximises consumer welfare. Hence, using Adam Smith's argument, by producer and consumer both following their own rational self-interest, the invisible hand of the market ensures material welfare and just distribution. This dominant model of marketing therefore assumes that the role of the marketing function is technical rather than moral in nature - its purpose is to translate demand into production, not to legislate on what demand or production might be 'good'.

There have however been a constant stream of criticisms of this classic marketing model, some of which have called into question the legitimacy of its moral basis; it is these that are discussed next. To begin with, the functioning of marketing system in practice is examined, and it is discussed how and whether the consumer sovereignty model is a legitimate description of this behaviour. In this, the approach of Smith (1990) is followed: in constructing one of the more comprehensive contemporary analyses of morality and marketing, he begins by detailing a thorough evaluation of the consumer sovereignty model.

\subsubsection{The Functioning of the Marketing System}

Marketing is replete with the discourse of consumer sovereignty (Dixon, 1992; Knights et al., 1994; Christensen, 1995). Contemporary definitions of marketing and the marketing concept tend to be predicated on an implicit belief in the centrality of consumer sovereignty, emphasising the need for 'customer orientation', 'customer focus', 'outside-in perspective', 'customer-driven strategies', etc. The following, taken from one popular textbook (Kotler et al, 1996:15), is indicative of most definitions of the marketing concept, and is explicit in its 'outside-in' perspective: 
The marketing concept holds that achieving organizational goals depends on determining the needs and wants of target markets and delivering the desired satisfactions more effectively and efficiently than competitors do.

Moreover, this discourse of consumer sovereignty has by now permeated a broad range of theoretical and practical arenas of management in contemporary organizations (Du Gay and Salaman, 1992). It is however based on two critical assumptions: that needs originate from the consumer, and that firms identify and act upon these needs when translated into consumer demand (Galbraith, 1972; Dixon, 1992; Knights et al., 1994). Both of these have been criticised as empirically inaccurate.

With the publication of The Affluent Society in 1958, Galbraith (1972, 1977) provided an early, important, and influential moral critique of marketing. He famously argued that firms generate rather than satisfy consumer needs, claiming that the industrial system had assumed sufficient size and power to render the consumer helpless in deciding what and how much is produced. To Galbraith (1972), this represented the "revised sequence" - a categorical denial of the "accepted sequence" of consumer sovereignty which accords power in the economic system to the individual. Galbraith's contention was that, in effect, modern industrialised countries were witness to planned economies, ruled over by large self-serving corporations with little regard for the public interest. The massive expenditures on marketing were in fact cited by Galbraith as evidence for want creation on a huge scale. Also in the late 1950s, Packard's (1957) populist work, The Hidden Persuaders, brought moral concerns about the manipulation of consumer need through marketing to a still wider audience. With his stylistic mixture of conspiracy theory and 'pop' psychology, Packard sought to reveal how the "depth men" callously turned the hidden urges and 
analysis, organizational behaviour, accounting, consumer studies, and cultural studies, etc. This is particularly important given the concern here with distinguishing questions of morality from questions of ethics, and moving from a prescriptive to a descriptive mode of analysis (see 1.3.2).

Notwithstanding the limitations of the marketing ethics project in capturing the full substance of morality in marketing, it certainly provides some important conceptual underpinnings for a study such as this. Indeed, the basic structure of this literature review is derived from Robin and Reidenbach's (1993) breakdown of ethical questions in marketing. Their argument is that marketing ethics is basically concerned with two sets of questions: broad macroethical marketing questions which relate to the fundamental functions of marketing; and more narrow microethical marketing questions, which relate to the specific actions of individual marketers, marketing organizations, and marketing industries. More simply, and in a form more appropriate to the current study, these might be regarded as questions regarding, respectively, "what is morally relevant about marketing itself?" and "what is morally relevant about particular marketing practices?" The review begins with literature regarding the former, and goes on to discuss the latter. In so doing, not only are the moral criticisms of marketing considered, but so too are the practical and theoretical responses and defences forthcoming from marketing practitioners and academics.

\subsection{Marketing as a Moral Subject}

In recent years it has been far more common for marketing scholars to comment on morality in relation to specific marketing practices than in relation to marketing itself. Indeed, a cursory glance at the two most established marketing ethics textbooks currently available, Smith and Quelch (1993) and Laczniak and 
Murphy (1993) would prompt one to believe that marketing was hardly in itself a moral subject, but rather a collection of activities and issues which might or might not be open to moral debate. However, there is a considerable history of academic debate concerning the moral standing of marketing, much of it taking place outside of the marketing field itself. This section will begin with a brief outline of the moral basis of marketing before going on to review the criticisms and defences of this position as they relate to three critical questions: the functioning of the marketing system, the role of marketing in society, and the impact of marketing on society.

\subsubsection{The Moral Basis of Marketing}

The accepted moral basis of marketing is enshrined in the utilitarian ideology of the market-based, economic model of capitalism (Galbraith, 1977; Gaski, 1985; Robin and Reidenbach, 1987). Its role in society is essentially a distributive one, such that consumers can find and purchase the goods that satisfy their needs and thereby increase their material satisfaction. Effective functioning of the marketing system should therefore ensure that consumers' needs are identified and used as the basis for production decisions. This assumed primacy of consumer demand is reflected in the notion of consumer sovereignty - an analogy based on the premise that the economy is ruled and directed by the customer. It is this concept which forms the underlying rationale for capitalism ${ }^{6}$, and hence provides a ideological basis for marketing (Smith, 1990). Essentially

\footnotetext{
${ }^{6}$ It is beyond the scope of this study to look directly in any detail at the morality of capitalism. Though related, it is a different question to that which refers to the morality of marketing. For the purpose of the study, 'marketing' is intended to mean marketing in a capitalist economy. Hence, capitalist objectives can be seen to be a constraint on the moral possibilities of marketing (Robin and Reidenbach, 1993).
} 
frustrations of innocent consumers into blind desires for unnecessary and unwanted products.

In his early work Baudrillard ${ }^{7}(1981,1997)$ develops these ideas ${ }^{8}$, claiming too that "the freedom and sovereignty of the consumer are mystification pure and simple," (1997:72). Where he departs from Galbraith however is in his denial that consumer needs can be categorised as 'false' or 'artificial' if they are created by marketing, compared with underlying 'real' or 'natural' needs. He posits that, in terms of the activities of consumption, there can be no distinction, because consumers do not consume for use value or exchange value, but for sign value. Hence, all consumption is driven by a single basic force which is "the logic of social differentiation", i.e. the need to distinguish oneself through the purchase and use of consumer goods. Indeed, Baudrillard (1997:73) condemns Galbraith's "moralizing idealism" and his depiction of the individual as a passive victim of the system. For Baudrillard $(1981,1997)$ the important unit of analysis is not the individual need, but the system of needs; and it is the system of needs that is the product of the system of production.

Marketing then might not so much be regarded as creating need for individual products, but rather, in aggregate, as contributing to the capitalist culture which emphasises and rewards material accumulation (Baudrillard, 1981, 1997; Featherstone, 1991). This is an issue which is covered in more depth in section

${ }^{7}$ Referenced works are English translations; original publication dates are 1972 and 1970 respectively. It is worth mentioning that at this time Baudrillard employed Marxian and structuralist techniques of analysis, indicating a largely modernist perspective; this stands in marked contrast to his later, postmodernist/post-structuralist work (Kellner, 1989; Ritzer, 1997).

8 Baudrillard (1997) references Galbraith extensively, and indicates acquaintance with Packard's book. 
2.2.2 below as part of a discussion on the broader impacts of marketing in society. In terms of consumer sovereignty though, the question of the origin of consumer need remains problematic (Knights et al., 1994) - a point occasionally, though rarely, acknowledged in marketing textbooks (Dixon, 1992).

Even if needs were objective and existing prior to consumption however, for consumer sovereignty to be upheld, they have to be duly acted upon by companies (Smith, 1990; Dixon, 1992; Knights et al., 1994). Smith (1990) sets out the position that only under perfect competition will firms be passive to the demands of consumers. In practice, he suggests, this assumption clearly does not hold and therefore firms are likely to have discretionary power within the market. It is this then which perverts the connection between consumer sovereignty and the marketing concept: consumer sovereignty presupposes the firm as passive whereas the marketing concept presupposes an active, strategic role for the firm (Smith, 1990; Dixon, 1992). Kotler (e.g. Kotler et al., 1996:12) explicitly acknowledges that marketing involves the firm not so much in responding to demand but in managing it:

Marketing management seeks to affect the level, timing and nature of demand in a way that helps the organization achieve its objectives. Simply put, marketing management is demand management.

Knights et al. (1994) have analysed the financial services market to illustrate how profit and cost concerns can channel as well as limit the attention accorded to consumer need. Moreover, they argue that consumer indifference to the product category can prompt firms to create rather than follow demand, such as through "informing" and "educating" customers of new technologies, beliefs and ways of behaving. Indeed, the creation of new markets that do not yet exist, and 
of going beyond what the customer thinks s/he wants, is being increasingly touted as the pre-eminent marketing strategy of successful, forward-thinking companies (see Hamel and Prahalad, 1991, 1994; Martin, 1995).

Overall then, as Smith (1990) contends, we might regard absolute consumer sovereignty as more grounded in ideology than in empirical fact. However, he does suggest that it can, in some circumstances, exist to reasonably significant degrees. The ethical question in this respect then becomes one of determining what level of sovereignty is acceptable, a project which Smith (1995) himself has attempted to resolve. However, in doing so he applies a distinctly micro analysis and considers the individual marketing exchange - therefore this is considered in section 2.3 below rather than here. The discussion is continued then by returning to the macro-moral issues of marketing's impact on society.

\subsubsection{Impact of Marketing on Society}

It was suggested above that regardless of the issue of specific need for specific products, it can be argued that marketing socializes and conditions consumers into a general state of perceived need and 'enforced' materialism. Kangun's (1972) book Society and Marketing makes one of the earliest contributions to the debate from the marketing discipline, and his thoughts, set out in the book's preface (p.xiv), serve as a useful introduction:

Marketing activities have a formidable influence on the ethical and social values of society. Although it may be reasonably argued that such institutions as education, religion and government have a significant influence on shaping this country's values, only marketing commends the tremendous resources that are used to convey a single message, "consume." 
In this respect, the role of advertising has been particularly open to critique, with arguments emerging from a range of sub-fields such as business ethics (Chryssides and Kaler, 1993; Phillips, 1997), marketing (Greyser, 1972; Pollay, 1986), and cultural studies (Williamson, 1978; Davidson, 1992; Baudrillard, 1997). Pollay (1986) provides a comprehensive summary and evaluation of many of the pertinent arguments. Central to the criticisms of advertising, he argues, have been the identification of its role in the generation and perpetuation of ideologies of consumption, and more broadly in instituting in society an identification of consumption with happiness. Advertising can create deep social unease by instituting a perpetual desire for what one does not have, and by depicting the (unattainable) possibility of a life enriched by material goods. Moreover, advertising is said to romanticise and fetischise goods, encouraging the individual to transfer their emotions and sense of self and identity away from people and spiritual matters onto objects. Greyser (1972) suggests that many of these moral attacks and defences are inherently ideological in nature, rendering few conclusions value-free. This though is hardly surprising given, as Pollay (1986) wryly observes, that advertising is in itself so value-laden. Nonetheless, he regards these as important moral concerns, and as such remain in need of further consideration and research.

Phillips (1997) however argues that the question is itself mis-specified: it is not advertising or marketing that should be open to such moral debate, but capitalism itself. Baudrillard (1997) too depicts the culture of consumption as a logical result of the system of production. Indeed, to some extent the debate over the ethics of marketing's propagation of materialist values has been largely undermined by the recognition that consumer society or consumer culture are essentially inevitable and unavoidable in capitalist economies. This has shifted attention towards the (largely postmodernist) project of identifying and 
describing the morality (or moralities, or amorality) of consumer culture (e.g. Featherstone, 1991). Indeed, the recognition that consumption might have its own morality has opened up a whole new conceptual space for scholarly enquiry in this respect - a point which the 'marketing ethics' literature has yet to recognise?

Finally in terms of the social impact of marketing at a macro level, two more issues can be said to have preoccupied the moral debate. First, concerns over the ecological impact of marketing have come under considerable scrutiny. To some extent this has reflected cycles of social concern regarding the (natural) environment within industrialised countries. Hence, an initial spate of (predominantly US) 'ecological marketing' literature emerged in the early 1970s following the identification of links between business and environmental problems precipitated initially by the publication of Carson's (1962) Silent Spring (e.g. Kassarjian, 1971; Fisk, 1973; Kinnear et al., 1974). Following a relatively quiet period in the late 1970 s and the early-mid 1980 s, environmental issues again entered into public debate, bringing forth a yet more extensive and sustained stream of literature (e.g. Prothero, 1990; Peattie, 1992, 1995; Menon and Menon, 1997). Nonetheless, as Kilbourne et al. (1997) have observed, the majority of this literature has concerned itself with micromarketing issues relating to the consequences of individual marketing activities, rather than to macromarketing concerns with the role of marketing itself. Accordingly, as they argue, the impact of marketing on the environment through the perpetuation of ever-expanding consumption tends to be conveniently side-stepped or simply

\footnotetext{
${ }^{9}$ This is symptomatic of a more widespread failure of mainstream marketing literature to develop a critical perspective on consumption and consumer culture. This debate has taken place largely in the cultural studies and critical theory literature rather than in marketing journals.
} 
ignored in the green marketing literature. Since this study is to focus in particular on the environment, this issue shall be revisited in chapter 3 in order to develop a more informed and coherent position in this respect.

The second outstanding issue is that of ideological stereotyping, once more an issue that has particular resonance for advertising. The main thrust of the moral criticisms here is that advertising can perpetuate socially undesirable stereotypes of certain categories of person and lifestyle, such that, for example: women become either housewives or sex objects; certain body shapes become associated with health, beauty and happiness; families become purely nuclear; and racial minorities, handicapped people, and homosexuality become excluded from the picture of 'normal' life (e.g. Pollay, 1986; Chryssides and Kaler, 1993). In defence of such depictions, it is commonly argued that marketers no more than reflect the social norms of target audiences, and that the brevity and directness of marketing messages leaves no scope for social reform (Greyser, 1972; Advertising Association, 1993). This "cultural mirror" argument is criticised by Pollay (1986) who documents important arguments regarding the distorting effects that advertising can have on the culture that spawns it. He summarises the main criticisms regarding stereotypes thus (p.27):

Simplistic, symbolic stereotypes, chosen for their clarity and conciseness, serve as poor models and inhibit sympathetic understanding of individual differences. This position has been articulated in detail regarding the portrayal of women, but the problem is universal, as ads can reinforce stereotypes for not just the sexes, but also for races, ages, occupations, families relations. To the extent that these images are disrespectful or unworthy of emulation, they are socially divisive. 
These moral arguments then have been well rehearsed in existing literature. To some extent the debate has now moved on. Commentators have pointed to the increasing "de-massification" and fragmentation of society into a profusion of diverse cultural groupings and identities (Featherstone, 1991). Also, marketing practitioners have been entreated to increasingly offer specific marketing mixes to ever narrower market segments, even to the point of "mass customization" (Kotha, 1995). This means that previously excluded and mis-represented groups of consumers are now being directly targeted by firms, suggesting that their particular tastes, styles, and interests might be better researched and represented. This of course brings with it additional moral questions. For example, there is the possibility of simply replacing one undesirable set of stereotypes - e.g. that women are either housewives or sex objects - with another - e.g. that women should 'have it all' and be a housewife, and sex object and successful businesswoman (Williamson, 1986).

Indeed, the expansion of professional marketing into hitherto ignored lifestyles is in itself a cause for concern for some critics. Featherstone (1991) for example points to how the need for stylistic individuality leads the modern consumer to construct a particular lifestyle as part of a calculated life project of conspicuous consumption. Baudrillard (1983) warns of the loss of reality as 'real' lives, 'real' people, and 'real' sex become submerged under the saturating torrent of superficiality, signs and signification that constitute the world of advertising. Essentially, these arguments lead us on to question the proper extent of the marketing domain, and what role marketing should be consigned to in society. If marketing is the cause of social ills, should and can it also be charged with remedying or preventing them? How far should it attempt to do so? It is the literature relating to such questions that the discussion now turns. 


\subsubsection{Social Role of Marketing}

The question of marketing's social role is essentially a question of corporate social responsibility, namely what role should business play in providing private solutions to public problems (Mintzberg, 1983; Harvey et al., 1984). The arguments for and against the adoption and incorporation of social responsibility into business activity are by now well rehearsed (e.g. Davis, 1973; Mintzberg, 1983; Friedman, 1988), as are those in respect of marketing (Gaski, 1985; Robin and Reidenbach, 1987; Smith, 1990). Whilst the arguments against are fairly straightforward, those in favour tend to vary in scope and ideology. Therefore, a brief review of these arguments is justified before looking specifically at the social role of marketing.

It has been widely argued that there is an inherently contractual nature to the concept of social responsibility, and it is this, more than anything, which classifies it as a moral issue. In essence, this form of social contract defines the implicit and explicit terms by which society and its institutions interact; this contract must be acceptable for both parties if the arrangement is to be legitimate and ethical (Steiner, 1972; Donaldson, 1982; Cannon, 1992). Therefore, by affirming that the function of business is to serve society and not merely itself, the social contract provides legitimacy for both the business enterprise, and for the capitalist economy. Donaldson (1982:57) thus asserts that, "the most important application of the social contract ... is evaluation of the performance of productive organizations from a moral perspective," (emphasis added).

Although the notion of a social contract provides a moral perspective to the functioning of business institutions in society, the fact that such a contract is unwritten and usually implicit creates problems for identifying the form and scope of these responsibilities. These can be thought of as occupying different 
positions on a continuum. Friedman's (1988) famous paper arguing that business enterprises should only pursue social ends if they are consistent with profit maximisation goals sits at one of the continuum. Since its original publication in 1970, Friedman's paper has been the subject of considerable scrutiny and dialogue in countless subsequent publications, and it is argued that his ideas have been immensely influential in the education of contemporary business mangers (Smith, 1993a). Essentially, Friedman's argument centres around the notion that pursuit of social objectives for any reason other than for profit is in itself unethical since the manager (in economics parlance, the 'agent') should always act in the best interests of the firm's owners (the 'principal'). For Friedman, the only acceptable pursuit of social ends is where they contribute to the long-run interests of the firm. This has become known as the 'enlightened self interest' position (though Friedman (1988:90) regards it as a "cloak of social responsibility"), popularly sloganised now as 'good ethics is good for business'. Thus social ends might be pursued if they could be shown to, for example: improve the firm's public image and thereby provide a differential advantage in marketing; increase worker commitment and productivity by improving employee welfare; or improve local labour markets and consumer markets by attending to the needs of the local community. Such possibilities are discussed by Davis (1973), Mintzberg (1983) and Owen and Scherer (1993) among others, although statistical correlation between social responsibility and economic performance has yet to be affirmed unequivocally ${ }^{10}$ (Aldag and Bartol, 1978; Owen and Scherer, 1993)

A slightly different form of argument views social responsibility as based upon

\footnotetext{
${ }^{10}$ To some extent this can be traced to methodological problems. See chapter 4 for further discussion on this point.
} 
the stakeholder model of the firm. In this sense, the manager can be seen as an agent of a range of principals with various stakes in the firm. Therefore, it could be argued that the long-run interests of the firm are best served by satisfying these stakeholders' social expectations (Freeman, 1984; Greenley and Foxall, 1997). This is closely related to the enlightened self-interest position. Indeed, this argument can even be articulated to present social activity for the satisfaction of stakeholder groups as an attempt to demonstrate to government and quasi-government authorities (and therefore potentially powerful stakeholders) that business can 'keep its own house in order' thus legitimising the firm, and pre-empting attempts to increase the extent and form of regulation which impact upon it (Davis, 1973; Mintzberg, 1983).

A different argument developed from the stakeholder perspective is that it is a firm's moral responsibility to satisfy their stakeholders (Evan and Freeman, 1988; Clarkson, 1995) ${ }^{11}$. This obviously occupies a position closer to the other end of the continuum, where the social responsibilities of business are considered to be morally prescribed. This does not mean that profitable social activities cannot be included in a conception of social responsibility, but rather that it is necessary to examine the motives of the act. Chryssides and Kaler (1993:232) for example argue that financial gain need not be totally absent from social responsibility behaviours, but where profit is the "sole or predominant motive" (as in Friedman's position), they should be discounted. Thus, "only in its purest form - as an ethical position - can corporate social responsibility stand by itself" (Mintzberg, 1983:4). Therefore, in this scenario, a firm's social responsibilities are to minimise its negative impact, and to maximise its positive

\footnotetext{
11 Though these two arguments are based on different theoretical foundations, Donaldson and Preston (1995) show that both are legitimate versions of stakeholder theory, and that it is not uncommon for authors to combine them.
} 
impact, in social arenas in which it has direct influence (Wood, 1991), or even in society as a whole (Swanson, 1995).

These different perspectives on social responsibility have been reflected in the different approaches taken to set out and re-define the social role of marketing. The first significant wave of such studies, which appeared in the early 1970s, were mainly prompted by the various social and moral criticisms of marketing, or by marketers response to these criticisms (Lazer and Kelley, 1973; Levy and Zaltman, 1975). Consequently, there is a strong corporate self-interest perspective underlying much of this revised view of marketing. Kotler was probably the main protagonist in this stream of literature, setting out two main conceptual strands: social marketing (Kotler and Zaltman, 1971) and societal marketing (Kotler, 1972). These are considered below. The third main strand, green marketing is briefly introduced below, but will be considered in more detail in the following chapter.

\section{Social Marketing}

Social marketing is simply the extension of marketing knowledge, techniques and concepts into influencing the acceptability of social ideas and causes rather than just economic goods and services. Whilst not formally defined until the publication of their paper, Kotler and Zaltman (1971) recognised that social marketing had long been in use by marketers, and it formed an important part of Kotler's project to expand the domain of marketing to ever more applications and contexts (see Kotler and Levy, 1969). However, the social marketing project came to be represented by many of its protagonists as a promising response to criticisms of marketing's negative impacts on society. It was held up to illustrate that marketing could be, and indeed was, a useful and constructive force in 
society (see Lazer and Kelley, 1973).

Social marketing is by now a firmly established sub-field of marketing, and significant research has been applied in the case of political marketing (e.g. O'Shaughnessy, 1990), charity marketing (e.g. Schlegelmilch, 1988) and church marketing (e.g. Devlin et al., 1996) among others. Indeed, whilst Kotler has consolidated his central role in the social marketing field with a number of further publications (e.g. Fox and Kotler, 1980; Kotler and Roberto, 1989), many mainstream textbooks now include sections on marketing in not-for-profit organizations (e.g. Dibb et al., 1994), and a number of specialist journals dedicated to social marketing have emerged (e.g. Journal of Public Policy and Marketing). Whilst fairly widely accepted then as a legitimate practice in itself, at least in principle, social marketing has however provoked further lines of inquiry into its moral dimensions (Laczniak and Murphy, 1993). Laczniak et al. (1979) for example provide an important discussion of the ethical ramifications of social marketing, reporting evidence from a survey of various academics and marketing professionals. They found that social marketing was a double-edged sword, with significant unresolved accountability issues, and the potential for moral controversy. The possibility of marketing techniques being morally neutral was shown to be problematic in the case of social marketing since the social cause and the techniques could not easily be separated. Indeed, the persuasive impact of marketing identified in 2.2.2 and 2.2.3 might be particularly questionable given that the exchanges sought by social marketing might be regarded as more socially profound than the consumption of conventional goods and services sought by traditional marketing. Crucially, these problems were perceived as less significant by marketing professionals than by academics. More recently O'Shaughnessy (1990) and Devlin et al. (1996) have explored the practice of social marketing in particular contexts, 
highlighting the moral ambiguities that might accompany manipulation of the social cause itself (rather than just using promotional activities to "sell" it) in order to appeal better to consumers faced with a market of such causes.

\section{Societal Marketing}

The other strand of Kotler's attempt to conceptualise and legitimate marketing's social role, societal marketing, was initially set out specifically in response to the burgeoning 'consumerism' movement in the US of the mid-late 1960s (Kotler, 1972). Consumerism, defined by Kotler (1972:49) as “a social movement seeking to augment the rights and power of buyers in relation to sellers" was regarded by him as a positive force for establishing enhanced consumer sovereignty in the face of excessive seller power. Moreover, he claimed that if marketing recognised and embraced the "opportunities" offered by the movement, then marketing could maintain its legitimacy and (by implication) avoid the increased regulation that had followed previous such movements. Kotler's response was in the form of a revised marketing concept which he labelled the societal marketing concept (SMC). This called for marketers to provide in addition to the basic elements of the marketing concept - customer satisfaction and profitability - a third element, namely "long-run consumer welfare". By doing so Kotler was acknowledging that what was good for individual consumers might not be good for society (Levy and Zaltman, 1975; Dixon, 1992). In justifying and proselytising the SMC then, Kotler (p.55) articulated a mixture of ethical and self-interest exhortations, with the emphasis falling slightly on the former:

The addition of long-run consumer welfare asks the businessman to include social and ecological considerations in his product and market planning. He is 
asked to do this not only to meet his social responsibilities but also because failure to do this may hurt his long-run interests as a producer.

Whilst Kotler proposed that one way to adopt the SMC was through a "consumerist orientation", as in a general cultural attitude of customer concern, his main emphasis was on setting out the type of products that might or might not be appropriate. He claimed that in respect to short and long-term consumer benefits, there were essentially four types of products: deficient products which offer neither short nor long-term benefits; salutary products which have low appeal but high long-term consumer benefit; pleasing products which give high immediate satisfaction but may cause harm in the long-term; and desirable products which combine immediate satisfaction with long-run benefit. These are shown in table 2.1 .

Table 2.1: Kotler's (1972) Classification of Products According to the SMC

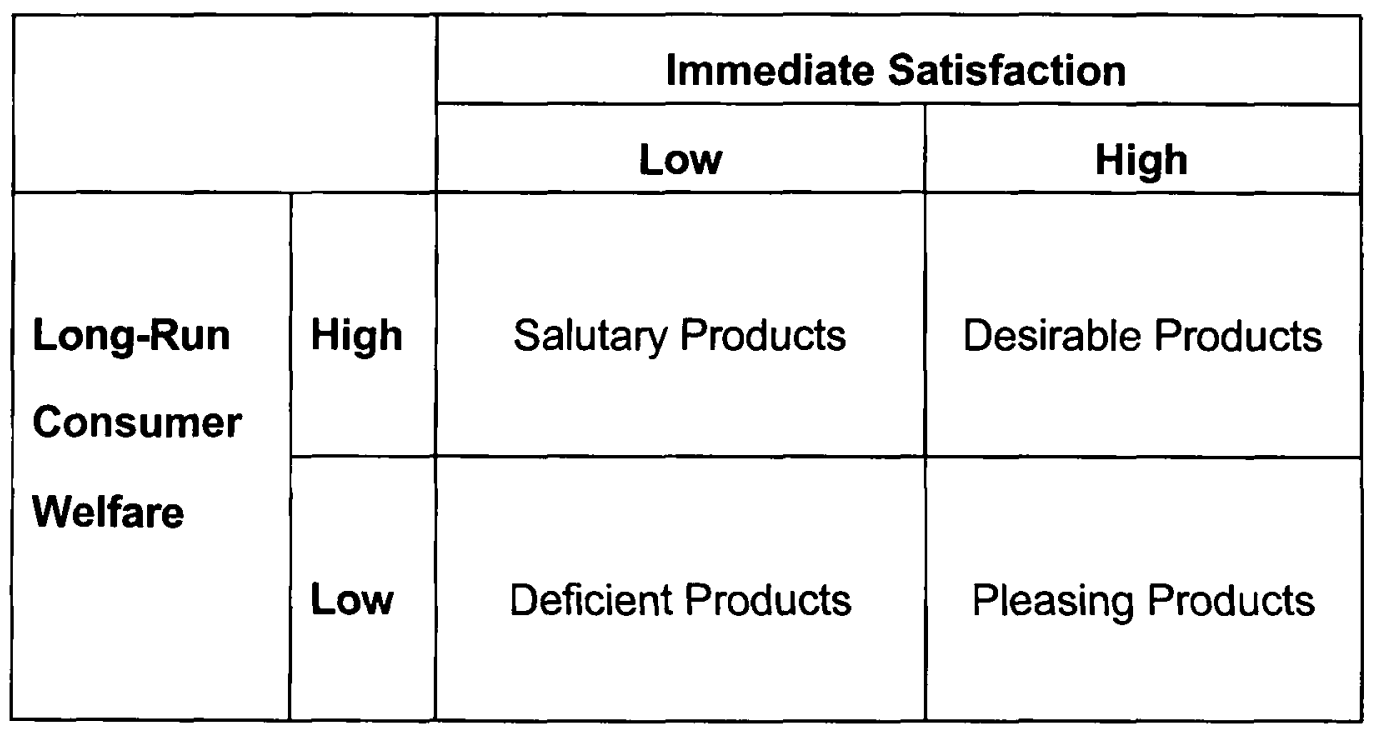

Kotler suggests that for the implementation of the SMC, desirable products should be produced, and that salutary and pleasing products should undergo 
product modification in order to move them towards the top right-hand quadrant; deficient products, he suggests, should be deleted from the product range.

Since this original formulation, the SMC has to some extent become an accepted part of the marketing lexicon, and appears in many contemporary textbooks, usually in terms of being an evolutionary step forward from the marketing concept $^{12}$. However, as Dixon (1992) observes, it is usually noted but essentially ignored during the main part of these texts. This, he claims (p.116), often distorts macromarketing issues by repeating and reinforcing "the myth that the pursuit of profit by marketing managers maximises social welfare". Some further studies have however been conducted. Gaski (1985) provides a criticism of the SMC with a basic re-run of Friedman's argument that marketers should not be expected to determine what is in the public's interest. Abratt and Sacks (1989) report on an important, and indeed rare, empirical study of the SMC. They trace the response made by companies in the South African tobacco and liquor industries to social campaigns against them, revealing a reluctance to accept social concerns in the former and a relatively conciliatory, constructive approach in the latter. They conclude that the liquor industry could be said to have adopted a societal marketing type response and that, as a result, appeared to be legitimating itself better in society than the tobacco industry. Finally, Prothero (1990) sets out the SMC in relation to social responsibility, suggesting that in the context of increased consumer concern over environmental issues, firms were responding with the introduction of the SMC, rather than the impetus coming internally from the firm's leaders.

\footnotetext{
${ }^{12}$ This is the case with all of Kotler's texts. Its appearance elsewhere is to some extent due to the sizeable impact that Kotler and his texts have had on mainstream marketing thought.
} 
There are still then a number of important issues which remain problematic in terms of the SMC. First, existing conceptions tend to assume that what is good or bad for consumers in the long-run is an objective, value-free fact. This disregards the possibility that competing positions may exist in a pluralist society and that these will have important implications for the identification of deficient, pleasing, salutary and desirable products. Indeed, Abratt and Sacks (1989) argue that many, if not most, products can be regarded as harmful or dangerous in some way, e.g. motorcycles are dangerous to drive, and high cholesterol foods are deleterious to health. By the same token, the manufacture of weapons can be argued to contribute to the peace process, and wind-powered turbines might be regarded as inefficient and a pollutant of the aesthetics of the local environment. Second, the SMC can be regarded as a somewhat blunt instrument since it takes no account of other moral issues involved in the marketing exchange, such as the rights of firms to market what they wish, and the rights of individuals to consume what they wish.

Clearly then, whilst the SMC might be a important moral arena of marketing, particularly in the sense that it provides a perspective on marketing as a legitimising practice, like social marketing it throws up its own complex questions of ethics. Therefore one of the main purposes of the current study is to develop and deepen the research agenda taken by Abratt and Sacks (1989), and thereby contribute some much needed empirical work relating to the practical implementation of the SMC. Of particular interest to this investigation then is whether - and if so, how - managers do indeed attempt to wrestle with these questions of the social good and the role of marketing in providing it. In the third section of the chapter that follows, this research agenda will be developed further. For having described the moral perspectives on marketing itself (i.e. the macro issues), it is time to turn towards considering morality in marketing 
practice (i.e. the micro issues).

\subsection{Morality in Marketing Practice}

In this section, attention is focused on morality in relation to particular marketing behaviours. The main concern is to identify which marketing practices, and which aspects of those practices, can and have been open to considerations of morality. Two broad domains can be identified in the current marketing literature: first, marketing decision making where considerations of morality are introduced during, and as a result of, the marketing decision process; and second, product augmentation where considerations of morality are applied in respect to their contribution to the desirability of the product. Each shall be considered in turn. Following this, attention will turn towards a possible third domain for analyzing morality in marketing practice, and indeed the focus for the current study, namely the construction and translation of moral meaning in marketing.

\subsubsection{Marketing Decision Making}

Considerations of morality in relation to the decisions made by marketers are certainly nothing new. Even before the concept of marketing became known and used $^{13}$ questions of morality have been associated with the way that buyers and sellers conduct exchanges, particularly in terms of faimess - fair prices, fair information, fair competition, etc. (Levy and Zaltman, 1975). Indeed, most marketing decisions are likely to have moral content of some form or another (Laczniak and Murphy, 1993). The relevant academic literature however only emerged in any quantity in the latter part of the twentieth century, again

\footnotetext{
${ }^{13}$ Bartels (1962) for instance places the initial use of the noun 'marketing' at around 1900.
} 
following public criticisms, influential publications such as Packard (1957) and Carson (1962), and the rise of the consumerism movement. This stream of literature is the one most closely associated with the 'marketing ethics' project. Three types of approach have been used - normative, descriptive, and prescriptive.

\section{Normative studies}

Normative studies are basically concerned with assessing which marketing decisions are ethical, and which are not. This can be in respect of the exchange itself, or any of the decisions and practices associated with the marketing process. Many, if not all, of these areas have been examined in one way or another in the existing literature, with coverage of issues at present expanding, particularly as new concerns and practices arise (Laczniak, 1993) .

Beauchamp and Bowie (1988) argue that ethics of marketing exchanges are principally centred around two key issues: freedom of choice in entering transactions, and the consequences of making those transactions. In terms of the first issue, De George (1990) claims that any market transaction might be considered fair or ethical if both parties have adequate and appropriate information and both enter the transaction willingly and without coercion. Conversely, freedom to choose can be said to have been restricted when there is evidence of undue influence, such as coercive behaviour or excessive manipulation, or informational irregularities, such as misleading or untrue claims, on the part of one of the agents of exchange. Clearly there are degrees of persuasion, and whilst some degree of persuasion may be acceptable, and clear cases of fraud may be illegitimate, De George (1990) emphasises that difficulties arise in making ethical judgements in more questionable areas such as deception 
and puffery. Issues concerning the legitimacy of promotional tactics such as over-claiming, misrepresentation, and misleading claims have thus received considerable coverage in both the marketing literature (Chonko and Hunt, 1985; Murphy and Laczniak, 1981) and the business ethics literature (Beauchamp and Bowie, 1988; De George, 1990). Ethical concerns related to freedom of choice have also been considered in relation to pricing and distribution decisions (see Smith and Quelch, 1993; Laczniak and Murphy, 1993).

In terms of the second issue, consideration of the outcomes of individual marketing transactions principally concerns issues such as product safety and fitness for purpose (Beauchamp and Bowie, 1988). Research investigating ethical issues in this sense has tended to focus on the harmful consequences of transactions, and in particular on analyzing specific high profile cases such as the Ford Pinto, Proctor and Gamble's Rely tampon, and Nestle's Infant Formula (e.g. Bowie and Beauchamp, 1988; Beauchamp, 1989). Whilst ethical concerns of this type have focused on the consequences to the individual of the marketing transaction, increasing social and environmental awareness has prompted investigation regarding the broader consequences of the exchange. For example, the environmental impact of products does not directly affect the individual consumer involved in the exchange, but rather affects society as a whole (Prothero, 1990).

Smith (1995) argues that these ethical questions relating to the individual exchange are essentially concerned with the extent of sovereignty granted to the consumer. He presents a marketing ethics continuum which delineates various types of exchange, depending on the extent to which either producer or consumer interests are favoured. Clearly, as Smith himself notes, this suggests, as was discussed in section 2.2.1 above, an inherent conflict between the two 
exchange parties, at least in the context of real markets and real exchanges as opposed to the theoretical models of economics. For Smith then, in the, as he puts it "ethics era" of the 1990s, customer expectations are for firms to adopt the consumer sovereignty position on his continuum. This demands that firms consider three dimensions of the exchange: the capability of the target market to make reasoned judgements; the availability and quality of information required for customers to make informed judgements; and the level of choice necessary for consumers to switch without prohibitive inconvenience and cost.

In addition to this literature related directly to the marketing exchange, other normative studies have focused on decisions and practices in the broader marketing process. This includes analyses of ethical issues relating to the basic 4P's - product, promotion, pricing, and distribution decisions (for reviews see Smith and Quelch, 1993; Laczniak and Murphy, 1993). Also, market research techniques have been examined (Akaah and Riordan, 1989), as have targeting practices, and in particular the ethics of targeting specific 'vulnerable' groups such as children (Beauchamp, 1989; Sharp Paine, 1993), ethnic minorities (Smith, 1995), and patients (Beauchamp, 1989).

Robin and Reidenbach (1993) have identified the predominant use of the 'grand narratives' of ethical theory - most notably, deontology, utilitarianism, and to a lesser extent, virtue ethics - in the normative marketing ethics literature. Correspondingly, they indicate a lack of consideration of the current debates of ethical postmodernism and moral relativism which, they argue, can make an important contribution to the discussion. In particular they throw doubt upon attempts to provide a unitary ethical theory for marketing, and propose in its place a moral relativist philosophy bounded by the constraints of society, capitalist objectives, and human limitations/capacities. In this way, they argue, it 
is possible to recognise the critical importance of history, time and context in the enactment of morally relevant marketing activities. From a different perspective, Smith (1995) also criticises the use of the philosophical grand narratives, but not because they are wrong or inappropriate, but because they are too complex and inaccessible for marketing practitioners.

These discussions by Robin and Reidenbach (1987) and Smith (1995) are important then in the sense that they throw light on the problems of applying ethical theories meaningfully to marketing problems, particularly when doing so from outside the arena in which they occur and are experienced. This has the effect not only of distancing the 'ethical' from the commonplace realities of marketing practice, but privileges the perspective and discourse of the judge over that of the judged (Parker, 1997). As it was argued in the introduction (section 1.3.2), it is the intention of this thesis to move away from such ethical judgements that are concluded from a lofty philosophical plane, and towards an understanding of morality located more directly in marketing practice. In the next section then, attention is turned to the descriptive literature.

\section{Descriptive Studies}

Descriptive studies are basically concerned with analysing how marketing decisions with moral content are conducted. According to Laczniak (1993:91), in his review of the state and development of marketing ethics research, attempts to model ethical decision making in marketing have represented "major advancement ... in terms of knowledge development". This principally descriptive project has been concerned with setting out the processes marketers use in reaching moral decisions, and in delineating the form and impact of various explanatory variables in determining the outcomes of those decisions. 
The first significant model was devised by Bartels (1967), who posited that decisions were a function of the types of ethical standards experienced by the decision maker, and the manner in which s/he complied with those standards. The former, he suggested, was composed of: basic cultural values inherited from social institutions such as one's family, school and church; standards learnt from these institutions which impacted upon one's economic role; and the expectations associated directly with one's economic role. The latter, he claimed was a matter of "ethical sensitivity", determined in part by the bases on which ethical obligations were determined by the individual.

Hunt and Vitell $(1986 ; 1993)$ presented a more comprehensive, and ultimately more influential model, the most recent version of which is shown in figure 2.1. This model makes more explicit the process by which moral decisions, and resultant behaviours might be made, with the avowed intention of constructing a base for empirical testing. The model begins with a set of contextual variables (down the left hand side of figure 2.1), namely cultural, professional, industry, organizational and personal characteristics. The main part of the model then attempts to integrate the major ethics grand narratives of deontology and teleology as different but complementary modes of evaluation undertaken by the individual. It is this evaluation which is pre-supposed as determining the consequent judgements, intentions and behaviour of the individual. Whilst some aspects of this model have been tested, these have not met with unqualified success, and various efforts are under way by Hunt and colleagues to provide more persuasive empirical support (Hunt and Vitell, 1993).

A number of other models of ethical decision making have also been conceptualised within the marketing field (Ferrell and Fraedrich, 1989) and more generally within the business and organizational ethics fields (Trevino, 1986; 


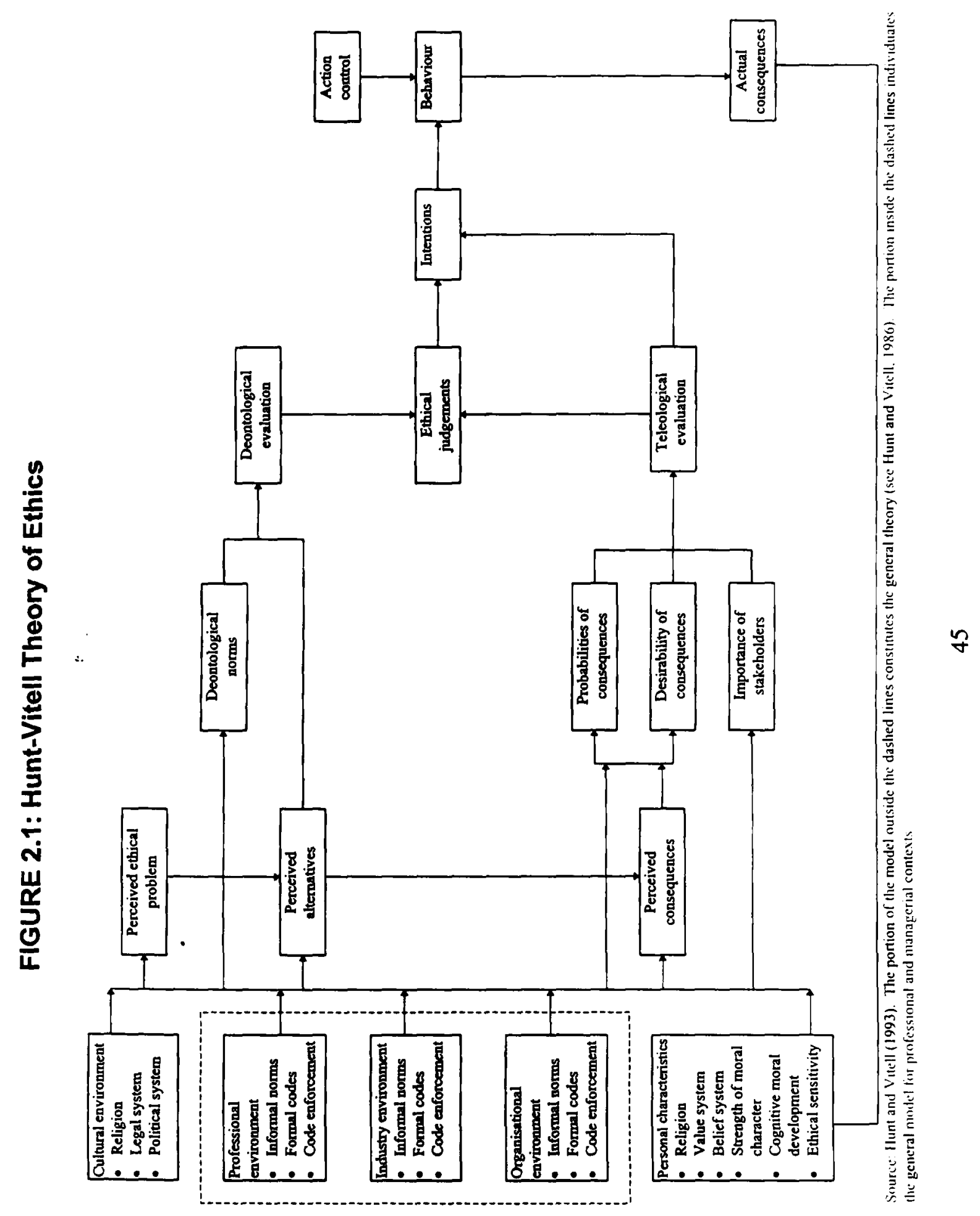


Jones, 1991). Central to much of this discussion is the relative impact of individual, contextual and situational factors on decision making with moral content. Trevino (1986) for example highlights the importance of individuals' moral development with situational factors such as the immediate job context, organizational culture, and work characteristics acting as moderating influences. In foregrounding moral development, Trevino draws heavily on the work of Laurence Kohlberg. According to Kohlberg (1969), moral development begins at the 'preconventional' level where the individual develops from a valuing of obedience for its own sake to valuing it instrumentally for their own immediate interest. At a higher 'conventional' level of moral development the individual moves from a valuing of interpersonal accord and conforming to stereotypical good behaviour to a stage where social accord and system maintenance take prominence. Finally, the 'principled' level is associated with stages where the individual values first, individual rights and social contract, and then universal ethical principals.

Situational factors are clearly also of considerable importance. Corey (1993) for example, has argued that certain conditions inherent to the marketing function tend to create an environment for marketing managers which is highly influential in prompting unethical behaviour. First, marketing can be regarded as a boundary spanning relationship aimed at completing transactions; hence the successful conclusion of deals may take precedence over other (moral) considerations. Second, marketers act as agents for their organizations and are therefore in a position to receive benefits from conducting successful transactions both as individuals (e.g., commission or bribes) and through the firm they represent (e.g., increased sales). Third, Corey argues that marketers have the capacity to wield significant economic power to influence, reward or punish particular customer choices. 
Of particular relevance however to the current study is the relatively recent rise in interest in the role of organizational culture in affecting ethical judgements. Aspects of organizational culture and climate have increasingly been the subject of academic scrutiny concerning morally relevant decision making in marketing and in more general business applications (e.g. Robin and Reidenbach, 1987; Waters and Bird, 1987; Sinclair, 1993; Vitell et al., 1993; Dahler-Larsen, 1994; Trevino and Nelson, 1995). Often this is centred around the notion of core values, namely the central and fundamental guiding principles about which corporate realities are organized and corporate endeavours are guided (Deal and Kennedy, 1982; Peters and Waterman, 1982). Core organizational values are proposed then to play a critical role in shaping decision making of all kinds, but because they contain a strong normative element, are thought to play a particularly prominent role in moral contexts (Starkey, 1998).

Other cultural elements, such as norms and behavioural standards, have also been argued to have a powerful influence on individual actions, and they can be seen to support behaviour which may be considered ethical or unethical to nongroup members (Turner, 1971; Jackall, 1988; Trevino and Nelson, 1995; Watson, 1994a). Individuals thus become socialized into certain accepted organizational behaviours which may prevail over (or replace) their personal (or previous) convictions or moralities (Van Maanen, 1991). For example, Posner and Schmidt's $(1984,1992)$ widely cited surveys reveal the rising incidence of managers claiming to have been forced into compromising their personal principles in order to conform to their organizations expectations ${ }^{14}$. Jackall's

\footnotetext{
${ }^{14}$ Indeed, there is a swathe of survey evidence of this kind which reveals a cynicism amongst managers concerning the ethics of superiors and peer groups (Trevino, 1986), with the work by Ferrell and colleagues (Ferrell and Weaver, 1978; Krugman and Ferrell, 1981) illustrating this most explicitly in the marketing field.
} 
(1988:6) study of US managers reveals that individuals "bracket, while at work, the moralities that they might hold outside of the work place or that they might adhere to privately and to follow instead the prevailing morality of their particular organization situation."

Culture is then in a sense an inherently moral terrain for it holds the key to the reciprocal relationships of obligation and responsibility - the moral rules and relationships - which define any particular community (Anthony, 1994; Watson, 1994a). For any social group, examination of its culture can reveal its values, ideologies, and moral rules (Schein, 1992). A recurring theme though in much of the descriptive literature which seeks to analyse the role of culture is the proposition that the right culture, the right values - i.e. an ethical culture, and ethical core values - can prompt improved ethical decision making from marketing and other staff (Robin and Reidenbach, 1987; Freeman and Gilbert, 1988; Trevino and Nelson, 1995). It is to these prescriptive studies that attention is now turned.

\section{Prescriptive Studies}

Prescriptive studies are basically concerned with suggesting how ethical decision making in marketing can be enhanced. Whilst there are a considerable number of prescriptive studies in the marketing and business ethics literatures, many normative and descriptive accounts also tend to include prescriptive elements. Given the scope and severity of social and ethical criticisms of the marketing function (and indeed of business generally), it is no surprise that considerations of how its ethics can be improved has attracted considerable attention. If we return to Hunt and Vitell's (1993) model of ethical decision making (figure 2.1), it is clear that the group of factors down the left-hand side (or some similar 
collection of factors) are those that might be regarded as the potential root variables for improving ethical decision making in this respect. If we disregard the broad cultural environment as relatively fixed, this leaves personal characteristics, and the professional, industrial and organizational environments in which ethical decisions might arise. Prescriptions have therefore focused on such issues as ethics training, (e.g. Sharp Paine, 1994; Trevino and Nelson, 1995), industry programmes, (Simmons and Wynne, 1993), corporate codes of ethics (Schlegelmilch and Houston, 1989), and professional codes of conduct (Diacon and Ennew, 1996a).

Most commonly advanced though within the prescriptive literature, and indeed often cited as necessary for those possibilities above to succeed, has been the culture management approach. In particular, authors concerned with improving organizational ethics have argued for the necessity of a managed transformation of the organization's values or culture (e.g. Stroup and Neubert, 1987; Robin and Reidenbach, 1987; Sharp Paine, 1994; Trevino and Nelson, 1995; Chen et al., 1997). Thus, Trevino and Nelson (1995:195) suggest that "organizations can proactively develop an ethical organizational culture, and that organizations with 'ethics problems' should take a culture change approach to solving them." Similar arguments are also present in relation to moral transformation in a wide range of sub-areas such as the environment (Elkington and Burke, 1989; Bernstein, 1992; Welford, 1995), strategy (Freeman and Gilbert, 1992) and governance (Diacon and Ennew, 1996a). Similarly in marketing, Robin and Reidenbach (1987:48) argue that "a major factor in developing successful socially responsible and ethical marketing programmes is management's ability to integrate ethical core values throughout the organization's culture," and hence, "the approach we suggest might be defined as the development or reformulation of a corporate culture." 
Essentially, the argument here is that since the organization's culture has such a significant impact on how individual's perceive moral questions whilst at work, and how they subsequently reach decisions and behave, it is this which has to be manipulated in order to improve organizational ethics. Though a different view of culture will be taken in this thesis (see 2.3.3), given our concern with aspects of organizational culture, it will be appropriate to deliberate on such prescriptions as the thesis is developed. Therefore, they will be considered in more depth in the following chapter - when the discussion has narrowed specifically to green marketing issues - since this will allow for a more focused assessment of this area of the literature. For the moment, the discussion turns to the second main area of micro-moral concerns, namely product augmentation.

\subsubsection{Product Augmentation}

The product augmentation area of the literature is that where morality is seen as having some impact on the purchase behaviour of consumers. Though this phenomenon has received relatively little attention from the academic literature, its import has long been recognised by practitioners. For example, it has been widely claimed that the UK Co-operative movement was established in the mid 19th century partly in response to customer concerns over the questionable ethics of existing retailers (Bonner, 1961; Birchall, 1994). Also, 'enlightened' labour policies on the part of large commercial enterprises during the early part of the 20th century were at the time seen to have afforded products an ethical dimension. As Ashley (1912:xiii) commented some 75 years ago regarding the benefits of Cadbury's labour policies:

It [the labour policy] has been a splendid advertisement. Instead of poohpoohing it for that reason, I think this is a particularly encouraging fact, and 
highly creditable to human nature. It shows there is such a thing as a consumer's conscience. The whole essence of the Consumers' League work in America and of the white lists of the Christian Social Union in this country [Great Britain] is to make it "good business" to be known to manufacture under satisfactory working conditions; and with increasing publicity and an increasing fellow-feeling among all classes, I expect that this is going to be the case more and more.

The assumption here is that consumers may incorporate moral considerations directly into their purchase decisions, and that firms can enhance the marketing effort by 'improving' and/or publicising their stance on certain morally relevant issues (Owen and Scherer, 1993). Smith (1990) provides one of the first, and more scholarly accounts of the subject. Using Levitt's (1980) notion of the augmented product concept ${ }^{15}$, he shows that various aspects of morality can contribute to the overall meaning of the product in the consumer's eye, and can in turn affect the consumer's desire to purchase the product. Whilst Smith considers only the negative impact of undesirable moral choices, there is clearly the potential for both positive and negative augmentation in this respect. Moreover, such augmentation can pertain to moral aspects of any number of issues relating to both the product and to the supplying firm. Hence, the product might be regarded as composed of - in addition to other 'conventional' attributes - a bundle of positive and negative moral augmentations. For example, Body Shop shampoo could (hypothetically) be positively augmented by the company's stands on fair trade and recycling, and the product's lack of animal testing and

\footnotetext{
${ }^{15}$ This basically says that different levels of the product can be identified: the core product which is the fundamental benefit sought by consumers; the expected (or actual) product which is the basic physical product which delivers those benefits; and the augmented product which is the addition of unsolicited extras which provide additional benefit to the consumer.
} 
inclusion of natural ingredients; by the same token it could be negatively augmented by the company's record of employee relations, and the product's use of non-renewable petrochemicals. The possibilities in fact are endless. Clearly then, augmentation of this kind is dependant on the perspective of the individual consumer, and on the actual attributes of the company and its products. The literature relating to these two aspects is reviewed next.

\section{Consumer Issues}

Various labels have been applied to consumer involvement with moral product augmentation, such as socially conscious/responsible purchasing (Anderson and Cunningham, 1972; Drumwright, 1994), ethical purchase behaviour (Smith, 1990), and ecological/green consumption and purchasing (Kassarjian, 1971; Prothero, 1990; Bohlen et al., 1993; Schlegelmilch et al., 1996). Broadly, these studies have sought to develop measures for such concerns, and thence to reveal who these consumers might be, what their concerns might be, and how these concerns might impact upon purchase behaviour. However, whilst it has been shown that some consumers certainly do at times incorporate moral concerns into product evaluations, further investigation into the phenomenon has provided little additional insight. Indeed, the avowed intention of many of these studies to segment consumers according to their moral evaluations, and ultimately to predict purchase behaviour - have yet to be properly realized. Whilst a considerable base of considered research is at least now beginning to emerge, particularly in relation to environmental issues (e.g. Drumwright, 1994; Shrum et al., 1995; Schlegelmilch et al., 1996), the main problem that remains is that many studies are mis-conceived in the sense that moral augmentation is rarely considered in relation to other 'conventional' non-moral product attributes. Also, the relationship between product and company associations is critical to an 
understanding of moral augmentation. The recent empirical study by Brown and Dacin (1997) is therefore significant for it provides evidence that what consumers know about a company does influence their evaluations of that company's products. However, though found to be a significant influence, the company's reputation for social responsibility was found to have less impact than a reputation for corporate ability or expertise.

Whilst studies of this kind have a strong managerial perspective, there are clearly important theoretical issues at stake with the recognition of consumer evaluations and behaviours of this type. Indeed, some writers have attempted to link these consumer issues with the broader macro concerns covered in section 2.2. Smith (1990) for example shows how organized ethical purchase behaviour (in the form of consumer boycotts) can provide a social control of business. Importantly, this implies a greater role for the market in prompting social behaviour than for any sense of moral obligation or moral impulse felt from within. However, Smith is explicit that the degree of consumer sovereignty is critical in providing for this control; in the case of boycotts, he argues that this is largely dependent on the ability and willingness of pressure groups and the media to provide sufficient and accurate information to consumers. Taking a slightly different route, Prothero (1990) links green consumption with the SMC, suggesting that the revised marketing perspective should be adopted in the face of changed consumer demands. As with Smith, Prothero sees the market as an effective social control of business, though in her eyes one clearly limited by the short-term opportunism and reticence of producers. Nonetheless, her focus on the market suggests very clearly that she, like Kotler in the original formulation, constructs the SMC in terms of selfinterest as well as morality. Crucial to Prothero's argument then (and indeed many other writers in this stream of literature) is that consumer concerns with 
social and environmental issues are increasing, and will continue to do so, perhaps indefinitely - a relatively unsupported, untested, and perhaps historically naive claim. We will return briefly to look at the role of the consumer in influencing specifically the environmental performance of the firm in the following chapter.

\section{Producer Issues}

With the link between the firm's social and moral stance and various legitimacy/economic performance/market share benefits widely alluded to (though less convincingly tested) in the literature - hence 'enlightened selfinterest' - it is somewhat surprising that the marketing strategies and tactics adopted by firms in this respect has received relatively little scholarly attention. Most attention has focused on identifying and analyzing the use of social and moral themes in advertising and promotions (Lill et al., 1986; Phillips and Brown, 1993; Iyer et al., 1994), and to a lesser extent, the production of socially acceptable products (Prothero and McDonagh, 1992).

In relation to the former, the use of social and moral themes in organizational communications has been shown to have steadily increased (Lill et al., 1986), with the incidence of environmental themes in particular experiencing a sharp incline during the late 1980s (Iyer et al., 1994). Academic research, whilst more advanced in this aspect of the marketing of morality than in others, is still relatively slight, concentrating mainly on print and television advertising (Lill, et al., 1986; Phillips and Brown, 1993; Davis (J.J.), 1994; Iyer et al., 1994) and social and environmental reporting (Harte et al., 1991; Gray et al., 1995a). As Brown and Dacin (1997) have shown, consumers' evaluations of companies' social reputations affects their product evaluations. Perhaps unsurprisingly then, 
one of the principal aspects of socially and morally oriented communications appears to be a strong corporate image theme. Iyer et al.'s (1994) investigation is, to date, perhaps one of the most comprehensive attempts to decode such communications. Their sample of 95 'green' television advertisements, drawn from 1991-2, were found to pertain mainly to corporate image or consumer durables. Crucially, it was found that, "the most dominant strategy was to position the corporation as being green," (1994:296) and, "in this event, the vast majority $(57 \%)$ of the ads used management control and social responsibility as the basis of appeal in the message" (1994:297).

The question as to why organizations have sought to utilise marketing communications in order to present an image of social responsibility, and what the social and organizational implications of such activity might be, have however been treated relatively superficially by marketing academics. The rhetoric of the marketing concept and the SMC pervades most accounts, eliciting from them an assumption that socially-themed communications simply occur in response to the demands of socially and ethically concerned consumers. A more critical perspective on social and environmental communications is however in evidence in the accounting field where there is a considerable literature relating to social and environmental reporting (Gray et al., 1995a). Most notably there is an interesting and important debate questioning whether social reporting occurs: as a pragmatic response to demand (Gray et al., 1993); in order to maintain public legitimacy (Patten, 1992); or as part of an attempt to re-enforce dominant corporate ideologies and avoid institutional change (Puxty, 1986). One of the intentions of this study then is to provide a similarly critical analysis of the more general area of moral product augmentation, and thus to provide a more explicit marketing perspective in this area. 
This completes the main review of the literature relating to marketing and morality. Every attempt has been made to cover the key issues and to report on the important contributions to the literature from various disciplines and subdisciplines, and from various perspectives and world-views. However, in assessing this literature it is evident that substantial gaps still exist in relation to our knowledge of marketing and morality. In the final section which follows, the research agenda of the current study is set out, with the intention of showing how it can provide an important, and much needed, contribution to this field of inquiry.

\subsection{Towards a Research Agenda: Exploring Moral Meaning in}

\section{Marketing}

Although there are a number of areas in the marketing and morality literature which might be fruitful avenues for further research, this thesis seeks to develop a perspective that has yet to be applied to any extent in this area, namely exploring moral meaning in marketing. Whilst the ethics of marketing and its various practices has been analysed, assessed, and diagnosed; and whilst explanations and remedies have been devised and tested; there has been little, if any, examination of what morality might mean, and how it is given meaning, in the context of the marketing process for the people actually involved in carrying out these activities.

Why is this important? Well first, most of the existing studies of morality in marketing begin with the assumption that the issue or decision is open for moral reasoning and debate. But in terms of the realities of marketing practice, this ignores the very important dynamic that leads to the identification of this issue or question as moral in nature (or not) in the first place. Hunt and Vitell (1986) 
give passing reference to this in their model of ethical decision making, suggesting that some level of "ethical sensitivity" on the part of the decision maker will determine whether an issue is subjected to moral reasoning. However they do not explore this issue further. But how can ethical decision making be modelled when it is not known with any certainty whether and how particular issues and decisions are granted moral status? Indeed, what does it mean to say that these are moral issues at all if it cannot be said with any certainty that they are experienced as such in practice?

The second problem with the existing literature is that macro issues have been subjected to relatively little empirical research from the marketing subdiscipline, and that macro and micro issues are rarely integrated very well. Clearly, questions of morality have been shown here to be of relevance for practitioners across a range of micro and macro issues, particularly in the context of growing social concern and scrutiny regarding their activities. So which moral issues are considered the most pressing and relevant? How do organization members deal with questions of marketing's social role? What makes them do so - is it personal responsibility, or the influence of managers, customers, and other stakeholders, or organizational norms appropriate to their work-role? And how are moral considerations reconciled with conventional marketing objectives? Until such empirical questions can be answered it will not be possible to set out anything more than claims of how marketing should take incorporate a moral dimension, rather than how it actually does.

Moreover, by looking at where morality has meaning in terms of the marketing process, it should be possible to provide some much needed linkages between the various elements of the literature discussed in this chapter. For example, is moral augmentation linked to moral decision making, or simply 'conventional' 
marketing decision making? Does it reflect a particular moral perspective on the social role of marketing? By identifying if and how areas such as augmentation, decision making, and social impact are experienced as moral questions during the marketing process, a solid basis for meaningfully relating them can be developed.

Whilst such questions at those posed in the previous paragraphs appear to demand a descriptive research agenda, the third criticism of the existing literature is that studies in this area to date which have been descriptive (and indeed most of this literature overall) have taken a very objectivist view of morality. Given though, as it has been shown, that morality is substantially influenced by situation, by context, and by culture, there is clearly a case for saying that organizational morality is in a very important sense sociallyconstructed (Jackall, 1988; Phillips, 1991; Brigley, 1995; Fineman, 1995; Parker, 1997). Jackall's (1988) study in the business ethics literature for example represents an important attempt to understand the working moralities of organization members (in this case, of US corporate managers). As he argues, organizational reality tends to induce a morality that is "contextual, situational, highly specific, and, most often, unarticulated" (1988:6). This view of morality as ambiguous and flexible is one which is in stark contrast to existing marketing literature, and one that is certainly in need of further exploration. Indeed, it can be argued that there are different moralities at work in the organization (Jackall, 1988; Fineman, 1995, 1997; Brigley, 1995). Fineman (1995; 1997) provides an important categorisation of these moralities, labelling them conventional, private, and enacted. Conventional morality, he argues, is the ideal morality espoused by the organization to various publics through formal communication; private morality relates to the personal moral positions of individuals; and enacted morality is the accepted moral rules-in-use of the workplace. Not only 
are these moralities all co-existent but each can present a different lens with which to view the moral terrain of the organization. It is clear however that the marketing literature has focused almost entirely on the former, i.e. the espoused, public, conventional morality of the organization, but has taken little account of the latter. This gives only a very partial picture of marketing morality. As yet then, we have little evidence of what morality actually means for those in the marketing process. If, as it has been argued, marketing is implicated in the transmission of certain value systems and ideologies through capitalist society (e.g. materialism), then surely some analysis of how morality is actually understood and communicated in and through the marketing process is a critical social project.

So what we have then is a broad range of research questions that simply have not been addressed to any extent in the marketing literature (or indeed elsewhere). Most importantly, this study seeks to describe the moral dimension of marketing, not in any conceptual or abstract way, but in terms of how the marketing process is actually enacted. What does morality mean for those involved in the marketing process? How are notions of morality given (or denied) expression and meaning in the marketing process? Answers to these questions are considered here to be essential in terms of developing a more informed view of morality in marketing.

\subsection{Conclusion}

The intention of this chapter has been to provide a broad overview of extant literature concerning issues of morality in relation to marketing. In doing so it has been necessary to introduce research from a fairly diverse range of areas, disciplines and sub-disciplines, in addition to that from marketing. It is believed 
however that the effect of this has been to strengthen rather than weaken the discussion, since many of the issues covered are of wider concern than to the marketing function alone, and the research in some aspects has been applied more broadly, more deeply, and at times more rigorously, elsewhere.

The first main part of the chapter sought to address the literature concerned with describing and evaluating marketing as a moral subject in itself. This covered studies regarding the moral basis of marketing, various moral critiques of the marketing system and of marketing's impact on society, and finally studies attempting to define and re-define the proper moral role for marketing in society. During this section of the chapter, important terms such as consumer sovereignty, social marketing and the societal marketing concept were introduced and discussed.

The second part of the chapter addressed the literature concerned with morality in relation to specific marketing practices. This involved discussing studies concerned with evaluating, modelling and enhancing the ethics of decision making in marketing; and then secondly, looking at studies where various aspects of morality were seen as a positive or negative product augmentation.

In the final part of the chapter, areas where the literature was considered to be under-developed or mis-conceived were identified. The focus of the current study on moral meaning in marketing was discussed in the light of these gaps in the literature and some initial research questions were set out. In order to develop answers to these questions, this investigation has adopted a narrower focus than 'marketing' in its general sense, and has concentrated principally on marketing in relation to the natural environment, i.e. green marketing. Whilst the selection of a specific case was precipitated mainly by practical concerns of 
being unable to easily say anything meaningful within the limitations of this study about such a wide area as marketing in relation to so many moral issues, the choice of green marketing to be this case was deliberate and calculated. The rationale for this will be set out in the next chapter where the concept of green marketing shall be introduced and the literature surrounding it discussed in terms of the moral perspectives commonly applied. 


\section{Green Marketing: A Moral View}

\subsection{Introduction}

Having set out the main morality domains of marketing in chapter 2, the purpose of this chapter is to focus more directly on the issue of the natural environment. This means that our concern with morality has narrowed now to a single, albeit fairly broad context; and hence, the domain of the study is similarly narrowed to consider only marketing activities that might take into account environmental dimensions. Such activities are refered to here as green marketing.

Though the consideration of environmental concerns in marketing was first initiated in the early 1970 s, it has arguably only really permeated mainstream marketing consciousness to any significant degree in the past decade (Menon and Menon, 1997). At present then the green marketing literature is still fairly limited, both in terms of scope and depth of analysis. Clearly though, it is growing quite rapidly and becoming a more established part of the marketing sub-discipline. Indeed, in addition to the published specialist green marketing texts (e.g. Charter, 1992; Peattie, 1992, 1995; Wasik, 1996), many marketing textbooks now include some mention of environmental considerations (e.g. Dibb et al., 1994 ), some texts devote a section to green marketing (e.g. Baker, 1994), and as well as a steady stream of articles in the major marketing journals and conferences, a number of journals have devoted special issues to the subject (e.g. Journal of Public Policy and Marketing, Fall, 1991; Journal of Advertising, vol. 24 (2), 1995; Journal of Marketing Management, forthcoming). In parallel with this academic interest, the practical implementation of green marketing in the marketplace could be said to have first occurred in a narrow sense from the early 
1970s onwards, become established as a more strategic and professionalised phenomenon from around the mid-1980s, and is now fairly well acknowledged as a legitimate mainstream business and marketing issue (Schot and Fischer, 1993; Menon and Menon, 1997).

To date, only little attention within the green marketing literature has been applied to understanding how exactly it might fit within a broader field of marketing and morality, and as yet there are no published reviews of the green marketing literature from a moral perspective. In its broader sense though, although green marketing is considered here as a subset of the marketing literature, it is clearly also strongly related to what might be referred to as the green business literature. Hence marketing is only one of a series of business areas in which environmental considerations can be analysed (e.g. Smith, 1993b; McDonagh and Prothero, 1997). Since some important consideration of the moral dimensions of greening has been made in this wider body of work, it is certainly useful to the extent that insights can be reasonably generalised to marketing. Moreover, it is obviously the case that morality has been most comprehensively explored within the business ethics literature, and so in this chapter (and indeed for the rest of the thesis) the green business and business ethics literatures are substantially drawn upon in order to develop a more informed understanding of green marketing and morality. This chapter begins then with a brief review of the existing green marketing literature and then sets out to explore green marketing from a moral perspective, principally with reference to the two major moral traditions in ecology - 'deep' and 'shallow' environmentalism. 


\subsection{The Green Marketing Literature}

In this section, discussion will focus on explaining what green marketing is, and evaluating the state of the literature surrounding the subject. In respect to the former, green marketing has generally been defined by marketing scholars as an expansion of conventional marketing. Thus, Peattie (1992:11) expands the Chartered Institute of Marketing definition of marketing to define green marketing as "the management process responsible for identifying, anticipating and satisfying the requirements of customers and society, in a profitable and sustainable way." In a fairly similar vein, Charter (1992:141) regards it as, "a holistic and responsible strategic management process that identifies, anticipates, satisfies, and fulfils stakeholder needs, for a reasonable reward, that does not adversely affect human or natural environmental wellbeing." It should be noted that both of these widely cited definitions claim either explicitly (Peattie) or implicitly (Charter) that green marketing is synonymous with sustainability ${ }^{16}$. Clearly then, under definitions such as these, few marketing activities as currently practised would qualify as green marketing. Therefore these types of definitions could be said to have identified green marketing more in terms of how the authors think it should occur rather than how it actually does occur. Indeed, rather than being specific to green marketing, this is largely true of both the marketing literature as a whole (see

\footnotetext{
${ }^{16}$ As Dobson (1996) shows, definitions of sustainability can vary immensely. The most widely cited definition is probably that of 'sustainable development' from the Brundtland Report (World Commission on Environment and Development, 1987): "development that meets the needs of the present without compromising the ability of future generations to meet their own needs." However, Dobson (1996) claims that sustainable development is ideologically loaded and should more properly be regarded as a sub-set of "environmental sustainability". This can be read as a more general appeal to environmental futurity, social welfare and economic equity. Unless indicated otherwise in the text, it is this conception of sustainability that is meant in this study.
} 
Brownlie et al., 1994) and the green business literature as a whole (see Newton and Harte, 1997). As Brownlie et al. (1994) suggest then, and as it is argued in the introductory chapter (section 1.3.1), a more descriptive, processual, and analytical perspective is adopted here. Hence, 'green marketing' is used in this study to refer to any marketing process where some account has been taken of environmental concerns. As was also made explicit in the introductory chapter (section 1.3.3), this means that no pre-judgement of the extent or value of that account taken of environmental concerns is necessary for including a particular activity within this definition. Whilst there are clearly more-green and less-green marketing activities, if some consideration of the environment is present, then for the purpose of this study, all activities will be regarded as within the purview of green marketing.

The current literature on green marketing is fairly limited, but has to some extent considered the perspectives of both buyers and sellers. In the case of the latter, broad analysis has been made of marketing strategy (e.g. Peattie and Ratnayaka, 1992; Wong et al., 1996; Menon and Menon, 1997) and the various elements of the marketing mix (e.g. Bebbington and Gray, 1992; Prothero and McDonagh, 1992; Iyer et al., 1994; Kilbourne, 1995). In terms of purchasing and consumption, most attention has focused on final consumers (e.g. Kinnear et al., 1974; Bohlen et al., 1993; Schlegelmilch et al., 1996), although there is a small literature emerging in relation to organizational buying (e.g. Drumwright, 1994) and supply chain issues (e.g. Lamming and Hampson, 1996).

Perhaps the most striking aspect of the existing green marketing literature is its clear lack of substantial empirical work (for a review see Siminitiras et al., 1997). With the exception only of the work on the environmental concern and consciousness of consumers, the majority of papers and texts have been 
conceptual in nature, and more often that not, highly prescriptive. Writing in 1994, Siminitiras et al. (1997) identified that empirical research was "very limited" in relation to green pricing decisions, applied only very narrowly to green communications, and "limited" in relation to distribution. Equally, the content (Menon and Menon, 1997) and process (Schot, 1992; Newton and Harte, 1997) of marketing and strategy development in relation to the environment have been argued to be have been, as yet, largely unexamined. Schot (1992:35) for example has contended that, "the way a business is run with regard to the environment is .... still largely a black box. Very little research is carried out into the way in which a company responds to the mounting pressures to prove their faithfulness and how they incorporate environmental aspects in their business strategy." Although Schot (1992) himself, and more recent work by Fineman (1996, 1997) among others, has begun to address this scarcity from a broad management perspective, very little quality research has emerged with a marketing slant (for an exception, see Drumwright, 1994)

Reviewing the more general green business literature (and in particular that concerned with environmental audits, culture and strategies), Newton and Harte (1997) provide an important and considered critique of the predominant style, methods, and approach of the green business writers. Not only are they highly critical of the quality and rigour of the work in this area, but also question the obvious attempts at "selling environmentalism" contained within it. The green business literature is, they contend, "highly evangelical" (p.77), "highly selective in [the] use of cases" (p.80), "somewhat outdated" (p. 87), "questionable" (p.88), and suffering from "a lack of a critical perspective" (p.88). In particular, they highlight the suspect manner by which the proponents of organizational greening attempt to persuade their readers of the rightness of their environmental cause, "selecting cases which neatly support their arguments, downplaying or ignoring 
those that do not ... Even among writers who adopt a more academic stance, there is still a marked tendency toward the 'little vignettes illustrating success' approach," (p.79).

Though not explicitly referred to by Newton and Harte, the green marketing literature is clearly implicated in this critique. Indeed, many of the exhortations to go green have been characteristically upped in the marketing literature with recourse to evidence of emerging and growing green consumer behaviours. Prothero (1990:96) for example has claimed that, "the greening of the British consumer has been increasing at a tremendous rate and is still surging upward," whilst more recently Schlegelmilch et al. (1996:35) suggest that, "the green market [is] expanding at a remarkable rate," and Menon and Menon (1997:51) identify, "a general consensus within the business and consumer communities that the environmental or so-called green market appears to be real and growing." That these claims are part of an 'evangelical' green marketing project is given credence by the recent contradictory evidence of declining environmental concern on the part of consumers, limited green purchase behaviour in many markets, and overall poor market performance of green brands and environmentally-labelled products (see Simonian, 1995; Wong et al., 1996). Such possibilities are on the whole overlooked, or conveniently ignored, by most green marketing writers. Nonetheless the appeals to go green in the face of consumer demand are in themselves particularly significant for the current study since, as it will be shown in section 3.4, they represent an important piece of the puzzle that is the morality of green marketing. However, before we go on to this, the rationale for selecting green marketing as a focus for this study of marketing and morality will be briefly set out. 


\subsection{Green Marketing as a Moral Domain}

Whilst many of the existing studies of green marketing make some mention of ethics or social responsibility, only very few have attempted to develop the moral dimension in any substantial way. Probably the most common perspective on morality adopted within the green marketing literature, although not always explicitly, is that the environment is assumed to be one of a set of social or moral issues to be dealt with by marketing. For example Drumwright (1994:1) argues that "concern for the environment is one example of a broader trend. Organizations are wrestling with how to incorporate a new and different type of criterion into business decisions - social responsibility." By the same token, in most business ethics textbooks the environment is usually included as one of the range of ethical issues that confront the organization (e.g. Chryssides and Kaler, 1993; Harvey, 1994), and environmental business concerns are often the subject of articles in the Journal of Business Ethics and Business Ethics: A European Review. This indeed is the basic starting point of this thesis: since it stands outside the economic and legal responsibilities of the firm, the environment is one of a number of social issues faced by marketing and is then, in itself, by definition, a moral issue. It should be recognised though that this is not meant to suggest that green marketing - either in theory or practice - is therefore necessarily a moral or an ethical concept; it is simply open to moral consideration because it involves the environment.

Any number of other moral issues that impinge upon marketing activities could have been selected for the purposes of this study, e.g. product safety, consumer privacy, targeting of children, etc. If the intention was simply to narrow the scope of the project, so too could any particular marketing practice have been selected (e.g. pricing, advertising, market research etc.), and then the whole range of potential moral issues relevant to this practice explored. The advantages 
of selecting the environment however are several. In a general sense, there are three reasons why green marketing as a subject is simply in need of further research. First, the environment has been widely argued (at least by green business writers) to be one of the more critically important issues confronting business and marketing today (e.g. Prothero, 1990; Peattie, 1995). Whether such assertions are wholly true or not, the environment is clearly both a substantial social concern, and a prominent contemporary business issue. Therefore, examination of the actual green behaviours of organizations should help in developing an informed perspective of its role in contemporary marketing practice. Second, the environment is a relatively new and under-researched addition to the marketing literature, and as such has been argued to be in need of considerably more research than is currently available (Menon and Menon, 1997; Siminitiras et al., 1997). Third, in the light of the criticisms of Newton and Harte (1997) identified above, the existing green marketing literature is in positive need of more in-depth, critical, and scholarly insight. This can also be viewed in the context of Brownlie et al.'s (1994) appeal for research in marketing to adopt a more critical perspective and to seek greater understanding of how marketing actually takes place. Hence, one of the main aims of this study is to provide a more informed picture of the process of green marketing, as it occurs in practice.

More specifically, for the purposes of this study, there are a further three reasons why the environment is considered to be a valid and important area in which to examine marketing and morality. First, the environment as an issue bridges both macro and micro moral considerations. In a macro sense it has been depicted in the literature as both intrinsic to a revised conception of marketing (Prothero, 1990; Peattie, 1995; Kilbourne et al., 1997), as well as inherently conflictual with marketing theories (Irvine and Ponton, 1988); in a micro sense it has been 
depicted also as an area in which the morality of particular practices and companies can be considered (Davis (J.J.), 1992, 1994; Drumwright, 1994). As such then, examination of green marketing allows us to explore morality very broadly as it relates to marketing. Second, the precise role of morality (if any) in green marketing practice needs to be clarified. It has been presented as both a purely technical process, with the environment simply another factor in the external business environment for marketing to incorporate into its standard models (e.g. Dibb et al., 1994; Kotler et al., 1996; Wong et al., 1996), as well as a distinctly moral one where marketing personnel might combine personal, emotional attachment to the environment with concerns for the social well-being of consumers (e.g. Prothero, 1990; Drumwright, 1994). Finally, the environment might be considered a good subject in which to examine the main concern of this study, moral meaning. Not only is the environment quite widely identified as a moral issue for business, but it is also currently very prominent in the media, as well as being traditionally viewed as a ideological battleground for different ethical beliefs. Hence, at the present moment, moral meaning in relation to the environment might be relatively prominent and easy to identify compared to other moral or marketing issues.

Having briefly outlined the green marketing literature and the rationale for this study's focus on it as a substantive field within the formal field of marketing and morality, we move on now to consider this literature in more depth, but this time from a more explicit morality perspective. As it was argued above, the attention to morality in the green marketing literature has been varied, and a number of different approaches can be identified. In this next section then we attempt to draw on the major ethical traditions in the green business literature in order to provide some kind of categorisation of these different approaches. 


\subsection{Moral Perspectives on Green Marketing}

The principal categorisation of moral perspectives used within the green business literature is that of 'shallow' versus 'deep' green positions. These largely reflect the two broad traditions within the environmental ethics literature of 'shallow' (or 'reform') ecology and 'deep' ecology. According to Naess (1989), the basic distinction between these two schools is that 'shallow ecology' tends to reflect a concern with fighting against resource depletion and pollution (the symptoms of environmental decline) for human-centred reasons (i.e. it is anthropocentric), whereas the philosophy of 'deep ecology' contends that the environment has a right to exist and flourish in its diversity irrespective of humans, and human needs (i.e. it is ecocentric), and that the structural and systemic causes of environmental decline should be tackled. Translated into green business, these ethical positions centre attention on: a promotion of environmentalism in the context of continued commitment to profitability and economic growth in the 'shallow green' movement; and more fundamental ideological change, new values, sustainable goals, and a transformed corporate morality in the 'deep green' movement. Since each perspective has a very different attitude towards morality, these two schools will used as a basis for discussing the green marketing literature in this section.

\subsection{1 'Shallow Green' Perspectives}

Most green marketing literature can be regarded as emerging from the 'shallow' green perspective ${ }^{17}$. According to Shrivastava $(1994,1995 a)$, this is the

\footnotetext{
${ }^{17}$ This is rarely acknowledged by the authors themselves. Only the 'deep green' writers tend to explicitly set out their ethical perspective. This is probably mainly due to the 'shallow' position being the dominant management paradigm, and thus may be perceived as demanding less justification than the more radical 'deep' position (see Shrivastava, 1994, 1995a).
} 
dominant paradigm in management theory and practice, and assumes that business organizations are morally neutral, rational, technological systems of production that serve the interests of stakeholders. Moreover, he contends that this perspective focuses on working environmental considerations into existing neo-classical economic models of free markets, maximisation of consumption, economic growth, and personal self-interest. In this scenario then green marketing is seen as a means of responding appropriately to consumer desires for environmental augmentation of some form. It is focused in the micro rather than the macro marketing domain (Kilbourne et al., 1997).

Green marketing literature that falls within this 'shallow' perspective can take a variety of positions in relation to morality. First, we have the largely managerialist studies which make no mention at all of morality, seeing it as largely irrelevant to the business of responding effectively to stakeholder environmental pressures. Wong et al. (1996) for example provide an important empirical study of green marketing strategies in practice. Their concern is to describe and evaluate the marketing of green products and not to undertake any consideration of the morality or otherwise of the tactics or the approach used. Similarly, Siminitiras et al. (1997) develop a useful review of the existing green marketing literature, and by utilising a 4P's framework to do so, side-step the possibility of considering any moral dimensions. Such studies then do not seek to question the moral basis of green marketing, nor indeed do they consider any moral questions in relation to micro activities; their main concern, as Siminitiras et al. (1997:431) state, is to aid in the development of more effective marketing programmes for business:

The likelihood of developing a successful green marketing mix increases with additional information and knowledge. Researchers, therefore, have a key role 
to play by generating empirically-based guidelines for designing, implementing and evaluating the various components of the green marketing mix.

A related (and often parallel) position looks at morality, but principally in relation to the moral considerations of 'ethical' and 'green' consumers. Into this category we can assign much of the environmental consumer behaviour literature (e.g. Bohlen et al., 1993; Dembkowski and Hanmer-Lloyd, 1994; Davis (J.J.), 1994; Schlegelmilch et al., 1996; Minton and Rose, 1997; Roberts and Bacon, 1997), as well as many strategy texts (e.g. Vandermerwe and Oliff, 1990; Peattie and Ratnayaka, 1992). Again, the avowed intention of these studies is principally to aid in the development of green marketing programmes such as to generate and maintain competitive advantage. A key argument in this respect then is that to remain competitive, firms must respond in an appropriate manner to the "green tide" (Vandermerwe and Oliff, 1990) of consumer and other pressures. A non-managerialist perspective however is provided by Smith (1990) who, though he does not focus specifically on environmental issues, examines the possibility of ethical purchase behaviour acting as a social control of business rather than simply being a phenomenon in need of an effective marketing response.

An extension of the ethical purchasing argument broadens the concern with strategic response to more comprehensive organizational change. Here, the concerns of customers and other stakeholders are considered to be sufficiently developed for them to necessitate more than just marketing responses, but adaptation of the very mission, strategy, structure and culture of the organization. Prothero and McDonagh (1992:164) for example argue:

The response of companies towards environmental issues in the future will be 
required to follow a number of routes which do not allow organizations to simply change a company's packaging, but rather to re-organize the whole structure of the firm... [and] integrate environmentalism into the overall strategy of the organization.

Equally, Welford and Gouldson (1993:147) argue that green marketing "is more than about changing the characteristics and advertising of a product. It must be part of an overall philosophy for business. And similarly, Elkington et al.'s (1991:121) advice for marketing professionals is that "environmental thinking must be integrated into every aspect of a company's operations. It must become second nature."

Such comprehensive change within the organization is presented as vital for two reasons. First, since the environmental performance of products is a function of various factors throughout its lifecycle (e.g. its raw materials, recyclability of components, production processes, by-products, packaging, transportation, product use, product disposal, etc.), the development of green products sophisticated enough to satisfy demanding consumers is seen as necessitating fundamental strategic re-orientation and re-organization (Peattie, 1992, 1995). Hence, the need for vision, mission and leadership are seen as critical elements in the drive for green competitive advantage (Elkington and Burke, 1989; Hutchinson, 1992; Peattie, 1995). Though this change in vision and values clearly contains some aspect of a concern for morality, the second reason cited for fundamental change does so more explicitly. Here the argument is based on the understanding that moral product augmentation relates both to evaluations of the product itself and of the organization supplying it (see 2.3.2). Accordingly, the satisfaction of green consumers is seen as requiring attention to the overall environmental reputation of the company (Davis (J.J.), 1991; Bernstein, 1992; 
Charter, 1992). Hence, with an ethical approach to greening viewed as an effective route to competitive advantage, this might be referred to as an instrumentalist moral perspective on greening.

The most central concern with morality in the 'shallow' green marketing literature is that of the sizeable group of studies which seek to provide normative assessments of the way in which various green marketing practices have been executed. These studies are 'shallow' in the sense that do not question the practices themselves, but rather the manner in which they are used (i.e. they are micro-moral concerns). Most common among these studies have been those concerned with misleading and deceptive green claims in green promotions and on-pack labels (e.g. Kangun, et al., 1991; Davis (J.J.), 1991, 1992, 1994; McCloskey et al., 1993; Kangun and Polonsky, 1995; National Consumer Council, 1996). Discussion has centred primarily around the use of terms such as 'biodegradable', 'recyclable' and 'environmentally friendly' which have been shown to have been used to describe unproven, inconclusive, or unrealistic product benefits. Whilst these studies clearly recognise and identify the moral dimension of such misrepresentation (the term "greenwashing" is frequently applied), they also tend to discuss the likely negative impact on consumers, and hence on marketing outcomes. Davis (J.J.) (1994) for example provides empirical evidence that consumers do indeed make ethical attributions and evaluations in response to green advertising claims, whilst the National Consumer Council's (1996) focus group study reveals a large measure of scepticism, confusion, and ignorance on the part of shoppers in respect to onproduct claims. The result, it is argued, is one of dented credibility for green marketers, and this in turn is seen as hampering future marketing efforts (Schot, 1992; Prothero et al., 1994; Kangun and Polonsky, 1995; National Consumer Council, 1996). In many respects, these and the studies discussed immediately 
previously are strongly related. In both cases, morality is seen to be important in green marketing because it is valued by customers and hence good for the business. Indeed, the title of Davis (J.J.)'s (1994) paper, "Good Ethics is Good for Business" could not be any clearer regarding the presumed role of morality in this context.

Occupying the most radical end of the shallow green perspective are those arguments which suggest that green marketing should be, or even is, based on moral principles of social responsibility and concern for the long-run welfare of consumers. Peattie (1995:29) for instance argues that, "environmental marketing is based on three principles: social responsibility, the pursuit of sustainability and an holistic approach." Equally, Prothero (1990) and Menon and Menon (1997) suggest green marketing should be based on social responsibility and hence, fall within the rubric of the societal marketing concept (SMC). This is slightly different from the positions set out above since here the concern is more with the social role of marketing (and hence macro-moral debates) than with whether particular marketing activities have been executed in an ethical or unethical manner.

Not infrequently, this perspective on green marketing is depicted as an evolved stage in strategic development. Hence both Schot and Fischer (1993) and Menon and Menon (1997) explain how the response of firms to green pressures has moved from total unawareness, to a compliance-based, "resistant adaptation" stage, to a more fundamental embracing or acceptance of environmental responsibility, and now (or soon) to a stage marked by manifest environmental concern, 'enlightened' and socially responsible management, and innovation-led (as opposed to compliance-based) solutions. Likewise, in categorising it as societal marketing, Prothero (1990) presents green marketing as part of 
advanced fourth period in evolution for basic marketing philosophies ${ }^{18}$. In all of these treatments, environmental concerns are still seen as opportunities to be exploited and therefore, providing the right strategic orientation is adopted, it is claimed that environmental objectives and economic objectives can be pursued in tandem. Although green marketing strategies might then be relatively risky, they are seen as the proper business of the 'evolved' entrepreneurial and socially responsible organizations (Menon and Menon, 1997).

Two characteristics distinguish this perspective on green marketing from the less overtly moral perspectives. First, it is argued that rather than being a consideration for various product components and attributes, environmental concerns have to determine which products are made, and whether particular products are made at all (Prothero, 1990; Schot and Fischer, 1993). Second, the reason these firms should pursue environmental objectives is not argued to be purely in response to consumer demand and other business environment pressures, but rather as a result also of internal factors such as a concern for social welfare, and a commitment to innovation (Prothero, 1990; Schot and Fischer, 1993; Menon and Menon, 1997). It is this addition of internal impetus, this assertion that green marketing will be conducted for reasons of social responsibility, which gives this perspective its moral dimension. Thus, firms will not merely respond to consumer pressures, but will develop solutions to problems not even widely known to consumers in order to take a lead in the

\footnotetext{
${ }^{18}$ Standard marketing theory, such as contained in any of the mainstream textbooks, presents basic marketing philosophy as developing in an evolutionary manner. This is said to have begun with a concern with production, moved on to a concern with selling, leading then to a focus on marketing. The societal marketing concept was conceived by Kotler (1972) therefore as the fourth stage in this evolutionary process. For a critique of this model of development see Houston (1986), or, more radically, Brown (1993).
} 
environmental movement (Prothero, 1990). However, this is no unreserved moral perspective: external pressures are still usually presented in these treatments as significant and increasing. Hence, although internal impetus is presented as essential, Schot and Fischer (1993:13) still contend that, "regulatory and public pressures will become more powerful ... New markets will become more articulated;" equally, Prothero (1990) and Menon and Menon (1997), as it has already be shown, argue, along with many others in the green marketing literature, that the growth of green consumerism is substantial and inevitable.

The reasoning here then is slightly confused and contradictory. What it seems to be suggesting is that, in some organizations, a previously non-existent social responsibility attitude has emerged (or is emerging) in response to external stimuli. However, once in place as a fixed cultural element of the firm, it is this attitude which underlies the emergence of green marketing practices beyond that actually demanded by these external forces - despite suggestions that these external forces will indeed continue to grow. So, in a sense, the firm moves from being a market-led, externally controlled system characterised by a degree of passivity to one which is rather more strategic, internally controlled, and functioning through managed flexibility (Christensen, 1995). Hence, it becomes a moral agent and affects a positive social role as a strategic response to indisputable and inescapable stakeholder pressures both current and anticipated. Newton and Harte (1997) argue though that such accounts are unconvincing. The evangelical fervour found in these treatments, they show, evinces a use of missionary language heavy in rhetorical appeals, implorations to change, and words such as 'must' and 'it is vital to...', but provides little account of exactly why and how firms might move from a reactive mode to one based on innovation and social responsibility. Such treatments can also be criticised for their lack of attention to the implications of suggesting this move from a passive 
marketing orientation to a more proactive societal marketing orientation. As it was shown in the previous chapter (section 2.2 .3 ), this turns responsibility for deciding what is in the long-term interest of consumers over to the firm. Again, there is little consideration of how or why this might be undertaken, despite representing a quite fundamental shift in marketing theory. Indeed, although some 'deep green' aspects have been introduced into these studies, it is their continued focus on existing marketing concepts and approaches - albeit expanded to incorporate environmental issues - that determines that they are 'shallow' as opposed to 'deep' perspectives on green marketing. We turn then now to this other main stream of literature.

\subsection{2 'Deep Green’ Perspectives}

We define a 'deep green' perspective on green marketing here as one which is concerned with institutional change, new management paradigms, and (at the very least) a pronounced scepticism regarding the power of the market to reverse environmental degradation. Probably the principal point of departure for a 'deep' conception of green marketing would be a rejection of consumption as a means of environmental amelioration. Since it is then in some respects an antimarketing position, the 'deep green' perspective on green marketing has mainly been set out in the green politics literature, although it has now begun to exact at least some influence on the critical green business literature. In this section we will begin by examining the main 'deep green' criticisms of green marketing, focusing on those which develop a moral argument, or an argument based around the role of morality in marketing; following this we will bring together those arguments in the green business literature which might be used to articulate a deep green morality for green marketing. 


\section{Deep Green Criticisms}

Implicit to the green marketing model is an assumption that environmental protection is possible in the context of consumption and economic growth. The 'deep green' position however denies the value of a constantly expanding economy, since such expansion ignores the inevitable limits imposed by finite ecological capacity, and is equated with bringing social and ecological malaise and structural inequality to industrial societies (Schumacher, 1974; Gorz, 1980; Irvine and Ponton, 1988; Porritt, 1984). Schumacher's (1974) important early exposition of the inadequacy of the assumptions for constant growth underlying the current economic model can be seen to have been highly influential to many of the subsequent 'New Economics' texts (Gray, 1992). Probably the key theme in Schumacher's (1974) argument in this regard is that the valuing of consumption over satisfaction as the basic unit of economic prosperity is fundamentally flawed. Thus, "since consumption is merely a means to human well-being, the aim should be to obtain the maximum of well-being with the minimum of consumption," (p.47).

In this scenario then, green marketing is essentially an oxymoron: no marketing activities can be 'green' since marketing is tied to the expansion of consumption. Indeed, many of the green politics texts unsurprisingly implicate marketing (and in particular advertising) as a nefarious element in the present economic system: "it disfigures landscapes and townscapes. It encourages waste... Its bottom line can only be environmental destruction," (Irvine and Ponton, 1988:63). Clearly the oxymoronic qualities of green marketing is a thorny issue for its proponents, as some of them acknowledge (e.g. Bernstein, 1992; Peattie, 1995). As yet though, relatively little attention has been paid to establishing what role marketing might have in a zero-growth economy. In the main, arguments have hinged on the development and marketing of 'sustainable' products (e.g. Peattie, 
1995; Welford, 1995) without accommodating 'deep green' demands for actively limiting demand. In one of a number of limited concessions to the 'deep green' position, Peattie (1995) for example does briefly explore the issue of "demarketing", acknowledging that it is an "unavoidable conclusion of environmental marketing logic," (p.38). Indeed he even succeeds in identifying one or two examples of green demarketing (p. 288), yet goes on to concede that, in practice, demarketing can principally be effected only through non-voluntary means, and undertakes no further discussion of the issue. Whilst Peattie then is one of several green marketing protagonists that acknowledges elements of the 'deep green' critique, his treatment is essentially 'shallow' in the sense that he examines the response that is possible within the existing social and economic structures of society. The 'deep green' position however suggests more fundamental change is necessary.

The work of Gorz $(1980 ; 1989)$ for example provides further illumination on the moral dimension of the 'deep green' critique of the green marketing model. He argues (1989) that within capitalism, systems of calculation and quantification, of economic rationality, have emerged as the privileged source of unquestionable certainty. Such economic rationality values and confers precedence on what is quantifiable - and in particular what is quantifiably efficient - at the expense of moral or aesthetic criteria. This, he argues, has given rise to a dissipation of notions of 'the sufficient' and 'too much' since there is no sense of 'too much' efficiency, nor therefore 'too much' quantity. In this scenario, parallel green projects in economics (Pearce et al., 1989), accounting (Gray, 1992), and pricing (Bebbington and Gray, 1992) aimed at valuing environmental criteria within the market economy can be criticised since these acts of quantification merely expand the limits of economic rationality within which 'enough' does not exist. For Gorz $(1980,1989)$ then, the issue is not so 
much how the sphere of economic rationality can be reformed, but rather how it can be limited; all reform is misguided since the market system privileges quantifiable criteria and marginalises (and ultimately degrades) the moral and spiritual dimensions of human interaction with the environment.

Arguing similarly, though in the context of environmental ethics rather than green politics, Tribe (1986) also criticises the impoverished morality of the green marketing model. He shows how the solution of environmental problems through the market is based on teleological reasoning, and therefore is essentially anthropocentric. According to Tribe, economic arguments tend to be couched in terms of the consequences for humans - such as environmental degradation is bad because it diminishes aesthetic satisfaction, or it denies resources to future generations, etc. - rather than deontological or duty/obligation terms, such as destroying or endangering plant and animal life is wrong per se, and/or humans have a duty to protect the environment. He argues that the effect of this reduction of environmental questions to human purposes acts to distort ecocentric human values originating in basic, nature-regarding impulses and obligations, and re-frames them as aspects of anthropocentric self-interest.

The third central concern of the 'deep green' critique is the emphasis in the green marketing model on individual consumer actions and orientations, what Smith (1990) regards as the "purchase votes" of social control. According to Sagoff (1986) it is inappropriate to treat environmental problems as economic distributive problems in this way: not only is there a very real problem in assigning a price to environmental attributes, but also environmental solutions are consequently predicated on consumers' willingness to pay. The problem with this, according to Sagoff (1986:229), is that individuals act differently as consumers than they do as citizens: "we act as consumers to get what we want 
for ourselves. We act as citizens to achieve what we think is right or best for the community." The danger then of approaching environmental problems through the market mechanism is the reliance on atomized individual consumer preferences which may reflect short-term 'selfish' desires and wants rather than a long-term appreciation of the common good. If consumers are not willing to pay for social and environmental 'goods' through their individual purchase behaviour, then such goods will not be supplied. In Sagoff's view then the 'public' and 'shared' nature of environmental problems renders them more appropriate for public policy than market solutions; equally, effective environmental solutions require consideration of moral and aesthetic principles and not just economic ones. Although the SMC model of green marketing attempts to deal with this issue by suggesting that the firm only provide "desirable products" (Kotler, 1972) which balance the immediate desires of the consumer, the profit interests of the firm, and the long-run welfare of consumers (see Prothero, 1990), this does not consider the problem of the consumer's willingness to pay for the additional 'benefit' at the time of purchase. Moreover, the emphasis still is on increased consumption to alleviate environmental problems - a proposition logically inconsistent with the 'deep green' perspective. In addition, Harte et al., (1991) also discuss the problem that conferring social 'votes' upon consumers through the market effectively disenfranchises the poor from environmental decisions. This is particularly problematic since 'deep ecology' theory places egalitarianism as one of its central principles.

Other principles of deep ecology are similarly ignored, or only partially incorporated, within the green marketing model. Gray (1992) for example argues that the principal contribution of the deep ecology agenda is 'soft' systems theory, and in particular the notion that in ecological terms everything is connected and interdependent with everything else. Whilst the green marketing 
literature has clearly embraced the rhetoric of systems thinking - and in particular notions of 'holism' - it is clear that much of this is in relation to the narrowly bounded sub-system of the organization rather than wider systems of the economy, society, and ecology (Shrivastava, 1995a). Where wider concerns have been incorporated, they tend to focus around constructs such as product lifecycle assessment - a typically quantitative, technocentrist, and teleological technique.

Whilst this represents a fairly brief review of the main 'deep green' criticisms of the green marketing model, it does suggest that to some extent the model might be inherently incapable of successfully incorporating the full range of green ideologies. Of particular interest to the current study, the focus on consumption, personal self-interest, quantitative and utilitarian analysis, and efficient, technicist solutions appears to marginalise morality from the purview of green marketing. We continue then with a discussion of the green business and green marketing literature where some attempt has been made to incorporate deeper green positions, and in doing so, possible elements of a model of 'deep' green marketing are set out.

\section{A 'Deep Green' Marketing?}

Most of the green business literature emerging from the 'deep green' perspective has been prescriptive in its approach, concerned mainly with setting out what a new green management paradigm would look like, and how organizations might adapt accordingly. The main importance of this literature for the current study though is its explicit concern with morality, and the delineation of moral concepts such as 'ethics', 'values', and 'spirituality' in its proposals for a new 'deep green' management paradigm. Shrivastava (1994:719) for example argues 
for an "ethical reorientation" of organizations and of organization studies; Welford (1995:125) contends that 'deep' greening requires organizations to adopt values based on "profits out, ethics in"; and the books by Stead and Stead (1992) and Davis (1991) both include whole chapters outlining the "small planet values" and "new value systems" necessary for re-alignment of organizations to ‘deep green’ sensibilities.

For the 'deep greens' then, environmental concerns require a response which does not simply reform the organization, but which demands a fundamental change in the organization's goals, values and beliefs. The primacy of growth must be replaced by a valuing of smallness and sustainability; profit goal systems must be replaced by ethical ones; anthropocentricism by ecocentricism; hierarchy and patriarchy by egalitarianism and democracy; and so on (Davis, 1991; Stead and Stead, 1992; Shrivastava, 1994, 1995a; Welford, 1995). As Newton and Harte (1997) show, this argument is frequently constructed around the concept of organizational culture, and is usually presented in terms of "selling" green cultures and promoting cultural transformation as the vital key to changing the organization. Though also found in the 'shallow' literature, there are numerous examples of this type of argument in the 'deep' green business literature: Welford (1995:114) for example argues that "the challenge facing the modern business is therefore how it can redefine and change its corporate culture in such a way as to be consistent with the concept of sustainable development;" whilst Stead and Stead (1992:186) argue similarly that, "it is necessary that the values for a small planet become the foundation of strategic decisions. This will require ... changing organizational cultures."

Such arguments for new business values and culture change have important parallels with those advanced with respect to enhancing ethical decision making 
which were considered in the previous chapter (section 2.3.1). Culture then is seen not only as the key variable in developing more ethical organizations, but also in generating a 'deep green' business ecology. Indeed, this latter contention is clearly based on the former, i.e. it is because culture is seen as a moral terrain that it is viewed as a means by which to introduce new values and normative beliefs into the organization. In this latter case however, the proposed change is yet more radical. As Newton and Harte (1997:87) contend, "green business writers have 'upped' the stakes since their aim is not just to change values within a commitment to profit maximisation, but to question the value of profit maximisation itself - a somewhat more challenging goal."

These arguments are based then on the assumption that the business organization can still be central to sustainable development and a 'deep green' economy, but only following radical cultural and moral transformation, and a spiritual reawakening within the organization. Whilst this 'deep' green business literature is still in itself under-developed, and largely non-empirical, it has clearly had only minor impact on the emerging green marketing literature. This is surprising in the sense that much of the 'deep green' critique focuses on aspects of consumption and consumer needs and wants, which of course are very much within the purview of marketing. Among the few exceptions to this has been work in the area of green communications by Iyer et al. (1994), Kilbourne $(1995)^{19}$ and McDonagh (1995), and by the latter pair in collaboration with Prothero in the macromarketing literature (Kilbourne et al., 1997). The main thrust of the 'deep' green communications literature has been to describe what might constitute 'deep' and 'shallow' forms of communication (to date,

\footnotetext{
${ }^{19}$ Kilbourne (1995) does not actually use the deep/shallow distinction but rather Green/green and ecological/environmental. It should be noted that his distinctions have been adapted such as to be consistent with those of the current study.
} 
principally advertising), and then to compare current practice with these models. These studies claim that a 'deep green' form of communication is indeed possible, and is: based on principles of trust, access, disclosure, dialogue (McDonagh, 1995); characterised by themes such as preservation, and the promotion of non-consumption or reduced-consumption 'alternative' lifestyles (Iyer et al., 1994; Kilbourne, 1995); and constituted by arguments formed from deontological and ecocentric positions (Kilbourne, 1995). In practice, this model of communication is argued to be only rarely in evidence, and limited mainly at present to non-commercial organizations.

A broader analysis of marketing in the context of the 'deep green' position is provided by Kilbourne et al. (1997). They argue that the failure of marketing theory and practice to respond adequately to 'deep green' criticisms lies in the sole focus of attention between directed at the micro- rather than the macromarketing domain. For Kilbourne et al. (1997) the ideology of consumption, and the privileging of consumption as the principal means for achieving happiness, success, and self-expression are the root causes of environmental degradation. They argue that since this ideology is rooted within the "dominant social paradigm" (DSP), i.e. the economic, technological and political structures of society, the approach to studying environmental problems in marketing should broaden to focus on marketing's role in delivering a transformation to "a new environmental paradigm" (p.16). Hence, they suggest (p.19):

Marketing research and reporting need to focus more on the role of macromarketing in conveying the deficiencies of the institutions within the DSP and on how it might influence the form of radical ecological change needed to transform those institutions. Academic marketers must question not only the 
$D S P$, but also their own existence.

Therefore, rather than merely acknowledging the potential conflict between 'deep green' positions and green marketing concepts as many other treatments do (e.g. Bernstein, 1992; Peattie, 1995), Kilbourne et al. (1997) seek to explain how this means that green marketing can not, and should not, be approached within the domain of micromarketing at all. Indeed, attempts to do so, they claim, will in the long run simply perpetuate and exacerbate the environmental problems they have intended to ameliorate. Kilbourne et al. (1997)'s project then is one of promoting "political and ethical deliberation" (p.19) amongst academics, practitioners, consumers and citizens, and of finding a place for marketing in informing society of its environmentally-destructive institutional make-up. Though the details of how or why this new economic paradigm is likely to arise are not fully explored, nor how or why macromarketing can prompt it to do so, this study is important for it explicitly redresses the lack of a rationale for marketing within the context of the 'deep green' perspective.

Shrivastava $(1993 ; 1994 ; 1995 \mathrm{a} / \mathrm{b})$ has perhaps more than anyone attempted to articulate an alternative 'deep green', or as he puts it, 'ecocentric' management paradigm, and he offers some tantalising possibilities for a 'deep' green marketing. Most notably, Shrivastava (1995a) re-envisions the exchange system to posit a model of industrial eco-systems. Based around the networks model of marketing (see Ford, 1997), he describes a model of inter-organizational relations "based on both the ecological interdependence and ecological performance of organizational communities" (p.127) whereby "a network of organizations ... jointly seek to minimize environmental degradation by using each other's waste and by-products and by sharing and minimizing the use of natural resources," (p.128). This is important for it provides substance to the 
'systems thinking' aspect of the 'deep green' perspectives, as well as offering strategic and relational dimensions to the 'deep' green marketing project. Critically, Shrivastava (1995a) combines his model of extra-organizational relationships with a concern for internal management goals, values, and practices. It is this linking between the internal and the external that the green marketing literature (both 'shallow' and 'deep') has yet to satisfactorily come to terms with, and which is one of the main aims of this thesis.

This concludes the part of this chapter concerned with reviewing the green marketing literature in relation to existing moral perspectives. We continue with a discussion of how these perspectives can be usefully brought together so as to consider them within a study of moral meaning such as this. To do so, the concept of organization culture is re-introduced and developed further.

\subsection{Morality in Green Marketing: An Organization Culture}

\section{Perspective}

As we have seen, a broad range of positions have been articulated in relation to the moral dimensions of green marketing, exhibiting varying levels of concern for morality, and focusing on both micro and macro moral domains. The shallow tradition says that morality is either a) incidental to green marketing, b) instrumental to its success, or c) strategically important in the context of stakeholder pressures; whilst the deep tradition says that morality either d) cannot be part of green marketing, or e) must be part of green marketing. Clearly then green marketing is a fertile area for consideration of morality in marketing, particularly since its role remains so ill-defined and disputed. The view in this study is that all of these different positions might have validity as a way of conceptualising the moral dimension of green marketing. Crucially though, 
whilst each of these positions presupposes a role for morality, none are based on extensive empirical evidence concerning the realities of the green marketing process. As it was stated in the previous chapter, there has as yet been little attempt to investigate how those actually involved in the marketing process understand and experience moral meaning, and it is true to say that this gap is equally, if not more, pronounced in the green marketing literature, where the moral issues are yet more complex. Indeed, the green marketing process itself remains largely unexamined (even outside the context of morality) and clearly demands more research attention. Without understanding how green marketing actually takes place, it is somewhat premature to suggest what role morality might or should have in that process. The major, basic goals of this study then are:

\section{1. to describe the green marketing process in detail; and thence}

\section{2. to articulate the role of morality in that process.}

In order to articulate the role of morality, it has been argued in the previous chapter that account must be made of Fineman's (1995) conventional, private, and enacted moralities within the organization (section 2.4). It is in these three domains that moral meaning can be established and communicated. Also, since the moral perspectives of organization members in relation to green marketing remains as yet unknown, all of the possible perspectives discussed above must be acknowledged as potentially providing a frame of reference through which that meaning can be interpreted. What we have then is a very complex yet fascinating area of enquiry. The view is taken in this study that the most effective means by which to explore this area is through the concept of organizational culture. As it was shown in the previous chapter (section 2.3.1), culture is an inherently moral terrain for it contains within it various shared 
values, norms, beliefs, ideologies, attitudes, and ways of behaving. As we have seen, organization culture can be identified as a major theme in the business ethics literature, the marketing ethics literature, and the 'deep' green business literature. Although in these treatments, it is principally presented as a means of changing morality, the organization culture concept is employed here as a means of looking at, interpreting, and understanding morality within the organization. Such a cultural perspective can take many forms, all of which drive us into the realms of "social significance - how things, events, and interactions come to be meaningful" (Smircich, 1985:63). The study of culture thus means exploring how the social world is constructed and how meaning is derived from it (Geertz, 1973). So organization culture is not only appropriate for studying morality (Brigley, 1995), but also for studying meaning (Geertz, 1973; Smircich, 1985). It is then a highly appropriate perspective from which to approach the current study.

Viewed thus, the key research issues are therefore the ways in which organizational members think about, interpret and communicate environmental principles rather than which 'type' of culture enhances ethical decision making, or prompts the most radical environmental solutions. The concern then is not with describing a culture, but in making a cultural analysis. Since the principal interest here is with morality, such a cultural analysis involves looking at cultural processes, cultural dynamics, and cultural phenomena which have moral relevance. In this sense then, we are following Pettigrew's (1979:574) view of culture: "a potentially more fruitful approach is to regard culture as the source of a family of concepts. The offsprings of the concept of culture I have in mind are symbol, language, ideology, belief, ritual and myth." It is then the process of developing and communicating cultural knowledge and meaning through various symbolic interactions that we are concerned with. This is not to say 
however that attempts to engineer a 'green' or 'ethical' culture should be dismissed as irrelevant, but rather that they are cultural processes in themselves and not the cultural process. This is equally true of claims that organizations have a certain type of culture, as well as attempts to espouse a particular green morality in formal, public communications: these are not the culture but cultural artifacts in themselves. Thus the espoused culture, or green image, can be regarded as a collection of symbolic phenomena, as texts, that require interpretation by employees, consumers and other stakeholders (Phillips and Brown, 1993). Hence, it is appropriate now to articulate the third basic goal of this study:

3. to set out the cultural dynamics through which morality is given (or denied) expression and meaning in green marketing.

Clearly, the cultural perspective tentatively outlined here permits a far broader understanding of the moral domain of green marketing activities than is usually envisaged in the literature. First, it allows us to consider the range of possible moral perspectives on green marketing rather than seeing any one as more compelling than the others; this gives voice to a wide range of interpretations, including those which may conflict with those of the dominant coalition(s) in the organization (Martin, 1992). Second, it makes it possible to bridge espoused, personal and enacted moralities in the workplace, thus lodging our understanding of morality securely within the deep structure of the organization (Brigley, 1995). Third, it allows us to consider internal organizational dynamics and external marketing communications as a single (cultural) process (Phillips and Brown, 1993).

This re-framing of organizational culture is clearly significant in terms of its 
methodological and epistemological implications, and it is these which are considered in the following chapter. We close the current chapter then with a few brief concluding comments on the main insights developed in this chapter.

\subsection{Conclusion}

In this chapter, the purview of this study's concern with marketing and morality has been narrowed down to the area of the natural environment. This has focused our attention on the literature relating to the theory and practice of green marketing. To begin with, a more inclusive, and less prescriptive, definition of green marketing was proposed. The green marketing literature was evaluated, and was argued to be relatively undeveloped, particularly in terms of a clear failure to provide serious academic treatment, critical perspectives, and scholarly empirical work. In the light of this, it was argued that one of the principal aims of this study was to contribute an in-depth, empirical investigation of how the green marketing process might actually be realized within the organization. This is to be used to provide a more informed theoretical basis for the green marketing literature.

The relationship between green marketing and morality was explored at length. The position adopted in this study was justified and this can be described as the selection of the natural environment as a particular area in which to focus the study of morality in marketing. However, the green marketing literature itself was found to exhibit a range of perspectives on morality. These were discussed in relation to 'deep' and 'shallow' green marketing, where the former principally considered morality in terms of micromarketing concerns and the latter in terms of macromarketing concerns - hence, to some extent, this section extended and focused the literature review of the previous chapter. The 'shallow' green 
literature was shown to be considerably more extensive than the 'deep' literature, but less consistent in terms of the proposed role of morality. Overall the green marketing literature was shown to have inadequately accounted for and incorporated the 'deep green' critiques and proposals. Consequently, this section of the chapter attempted to begin to redress this shortcoming, in the same way that, for example, Harte et al. (1991) and Gray (1992) have achieved in the critical accounting literature.

Finally, an organization culture perspective on green marketing and morality was set out. It was shown that the approach adopted here considered culture more as a way of looking at how meaning (in this case moral meaning) was established and communicated through language and symbolic action, etc. than as a unitary concept to describe the entire social context of the organization, or still less as a variable determining the level of ethical decision making in the organization. This view of culture was seen as providing a good way of providing a conceptual bridge between the disparate concerns of this study, i.e. the green marketing process and its outcomes, and the role of morality and of moral meaning in this. This organization culture perspective is developed in more detail in the following chapter where the methodological and epistemological issues relating to the study are discussed. 


\section{Truth, Knowledge and Method in Researching Morality in Marketing}

\subsection{Introduction}

The consideration of issues of morality, and more specifically the environment, within the marketing discipline has provided an interesting and potentially important stream of research. However, it is evident that the bulk of published work in this area consists of normative prescriptions and non-empirical models rather than critical, scholarly accounts of what marketers actually do in practice in this context. Moreover, the internal dynamics and cultural patterns of the marketing function in relation to the environment remain critically underresearched, particularly in respect to any moral dimensions. To date then, the majority of the evidence which has been used to support the many prescriptive, managerialist, and frequently evangelistic tracts prevalent in the business ethics and green business literatures has tended to be either brief and anecdotal sketches (see Bernstein, 1992; Clutterbuck et al., 1992; Smart, 1992; Peattie, 1995) or self-reported accounts (see Roddick, 1992; Chappell, 1993; Lager 1994). As Newton and Harte (1997:79) comment in relation to the green business literature, these treatments commonly draw on the familiar formula of Peters and Waterman (1982) where cases are carefully selected to support the author's arguments, and possible disadvantages and problems are strategically ignored. The need then for more rigorous research across these areas is pronounced and unequivocal.

But what form should this research take? In which way should the researcher 
engage with the research field in order to provide answers to the research questions set out in the previous chapter? Clearly, the success of this knowledgecreating process depends on the depth, quality, and theoretical relevance of the empirical research undertaken. Within the business ethics and green business fields there has been considerable criticism directed towards the quality of existing empirical work (Randall and Gibson, 1990; Gladwin, 1993; Bain, 1995; Newton and Harte, 1997). Many of these problems, it seems, can be traced to issues of methodology. Much of the empirical research conducted in these areas is characterised by survey work and other quantitative approaches which are firmly rooted in the positivist tradition of logical empiricism (Randall and Gibson, 1990; Brigley, 1995; Fineman, 1995). This approach, it has been argued, is in many important respects largely inappropriate for the research of moral dimensions in business (Brigley, 1995), and also, it is proposed here, for the empirical investigation of the questions posed in the previous chapter.

The purpose of this chapter then is to explain why this positivist approach may be inappropriate, and to set out why, and how, a more interpretive approach, based on a social constructivist view of organization culture, can be utilised to provide important empirical insight into marketing and morality. We begin then by outlining the epistemological and ontological foundations prevalent in existing empirical work in the field. In particular, the Burrell and Morgan (1979) framework delineating distinct and identifiable research paradigms in the social sciences will be explored and analysed. Second, the central issues which have particular import for methodological choices are identified and their implications assessed. Third, the methodological and epistemological implications of engaging in a cultural analysis of organizations is explored. And finally, in the 
context of this broader discussion, the research method applied in the current study is described and justified, with particular attention being focused on the overall research strategy and method, the data used, the procedures for analysis and theory building, and the steps taken to ensure validity.

\subsection{Epistemological Foundations}

The key question to which this chapter is oriented is: how should the marketing researcher describe reality, reveal truth, and contribute to knowledge in studying morality within the field of green marketing utilising an organizational culture perspective? It is evident that in order to achieve these goals, it is possible to bring to bear a whole array of qualitative and quantitative techniques; how they are used however depends upon the researcher's own conception of this reality which is to be described and analysed, i.e. their ontological assumptions. Not only this, but the knowledge thus derived also reflects the way in which these methods are selected and applied. Researchers then can be said to not so much conduct research, but rather to, "engage a subject of study by interacting with it through means of a particular frame of reference, and what is observed and discovered in the object (i.e., its objectivity) is as much a product of this interaction and the protocol and technique through which it is operationalised as it is of the object itself," (Morgan, 1983a:13). The 'truth' of any given research study is therefore inextricably bound up in its methodology.

The problem of developing an appropriate mode and method of inquiry is not however an abstract one. Not only is the researcher making implicit assumptions about what is important about the chosen field of inquiry, but s/he is also making a decision as to the way in which the reality of that phenomenon is to be 
interpreted. Thus, by considering research as engagement, the inherent relationship which binds the researcher's conception of the phenomenon being studied and the logic or strategy of the research itself is, in effect, made explicit (Morgan, 1983b). Starkey (1990:97) states the case succinctly: "the selection of a method of data collection and analysis determines the potential boundaries and depth of knowledge that can be generated. Choice of methodology, therefore, has major constraining or liberating potential."

Certainly then, no single method in management research should be regarded as superior per se. As Fineman and Mangham (1983:298) argue, "what concerns us in this search for the one, true method....is the notion that somewhere, somehow that 'truth' is accessible to those who mutter the correct imprecations and prescribe the proper magic circles on the ground." The view adopted here then is that management research might be best regarded as a craft (Miles and Huberman, 1994; Watson, 1994b). According to Watson (1994b), the crafting management researcher does not simply describe an objective reality that is observed as a scientist might, but then nor does he or she create a reality by seeing and knowing it as an artist might; in effect, the crafting researcher mediates reality through informed selection, interpretation, colouring, emphasis, and shaping of their findings. Thus, "we cannot see anything without a cultural and human mediation - but that is not to argue that there is nothing there other than that mediation," (S79). Seen in this way, it is necessary to consider both the most appropriate methodology with which to study morality in marketing, and to make explicit the philosophical basis upon which this decision has been made. It is this which we turn to next. 


\subsubsection{Paradigms of Research}

The nature of reality is not a commonly shared and understood notion. Morgan and Smircich (1980) have identified six distinct views that can form the basis of an understanding of reality, forming a continuum ranging from an extreme objectivist perspective to an extreme subjectivist perspective. These are shown below in figure 4.1. Burrell and Morgan (1979) conceive of methodology wrought from these differing philosophical perspectives as constituting different paradigms of organizational research. Of these, it is evident that by far the most

Figure 4.1: The Nature of Reality

\section{Objectivist}

Concrete Structure

Concrete Process

Contextual Field of Information

Symbolic Discourse

Social Construction

Projection of Human Imagination

\section{Subjectivist}

common in management research are the functionalist paradigm and the interpretive paradigm. 
According to Burrell and Morgan (1979) the functionalist paradigm rests upon the assumption that society is real, concrete, and systematic. Therefore, the functionalist paradigm relates to the upper half of figure 4.1. Research in the functionalist tradition will generally exhibit a methodology which is problemoriented, objective and value-free. The mode of inquiry here then is predominantly, but not exclusively, positivist - i.e. where the focus is on facts, and where measurable data is gathered from large samples in order to formulate and test hypotheses which can explain causality and fundamental laws (Easterby-Smith et al., 1991). Positivist research of this kind generally deals in quantitative data.

To date, this has been by far the most prevalent mode of engagement in conducting empirical research concerned with issues of morality and the environment in business and marketing. Indeed, in their review of the business ethics literature, Randall and Gibson (1990) revealed that $81 \%$ of available empirical studies relied exclusively on survey data - a classic positivist research instrument ${ }^{20}$. In general, survey work such as this has focused on establishing measures or proxies for corporate social responsibility or ethics based on the attitudes of individual managers (Ford and Mclaughlin, 1984; Diacon and Ennew, 1996b), and exploring the relationship between these attitudes and key organizational variables such as financial performance (Aupperle et al., 1985; Ullmann, 1985; McGuire et al., 1988), market share

\footnotetext{
${ }^{20}$ A recent Marketing and Social Responsibility special edition of the European Journal of Marketing (vol. 30, no.5, 1996) also provides a good example of the balance of research in the literature. Of six papers published, only two were based on original empirical work carried out by the authors - and both were surveys (Diacon and Ennew, 1996b; Schlegelmilch et al., 1996).
} 
(Owen and Scherer, 1993), executive success (Hunt et al., 1990), employees' hierarchical position (Collins and Ganotis, 1973) and employee commitment (Wood et al., 1986). Empirical research centred on the production and form of green marketing artifacts such as environmental products and adverts has also been predominantly survey-based (Lill et al., 1986; Prothero and McDonagh, 1992).

In contrast, interpretive approaches are based on very different ontological assumptions. The basic starting point here is that reality is socially constructed. Viewed thus, the social world is regarded as a product of intersubjective experience and exists only through the meaning that social actors apply to it (Hassard, 1993). Accordingly, there is not an absolute concrete corporate morality, but there is one that exists through the subjective experience of various actors and groups of actors inside and outside of the organization. It is the meanings that organizational actors apply to ethical principles and actions that become the research focus for studying morality in marketing within the interpretive paradigm. Research of this kind is thus subjective (therefore relating to the lower half of figure 4.1), yet reflexive. Furthermore, within the interpretive tradition, researchers most commonly engage their subject phenomenologically, i.e. the focus is on meanings, and theory is induced from holistic data gathered in depth and over time in a small sample of organizations (Easterby-Smith et al., 1991). This data is frequently qualitative in nature.

There is a small but growing body of work associated with interpretive approaches to social responsibility, business ethics, and green business with a number of researchers such as Harvey et al. (1984), and Fineman $(1996,1997)$ 
in the UK and Jackall (1988) and Bird and Waters (Waters and Bird, 1987; Bird and Waters, 1989) in the USA providing important works in the field. More recent work has begun to focus more explicitly on the marketing function (Abratt and Sacks, 1989; Drumwright, 1994) although it is clear that the discipline has still not yet embraced interpretive approaches to even nearly the same extent as it has the dominant positivist tradition. It is the intention of this study then to build upon these works and to contribute to further development of the interpretive turn in the marketing and business ethics fields.

Certain caveats exist however in categorising modes of inquiry in this way. Morgan and Smircich (1980) argue that certain research strategies are more appropriate for some social phenomena than others. This conforms with the view espoused by Blackler and Brown (1989) who contend that in the social sciences, the idea of incommensurable scientific paradigms (Kuhn, 1970) is not so much applicable as is the notion of multiple paradigms of practice. These are not necessarily mutually exclusive, and indeed they may even at times overlap. Hassard (1993:110) takes the argument even further, stating a case for multiple paradigm research: "each of the Burrell and Morgan paradigms is limited in its methodological scope. Thus the author feels that it is wrong to assert that any paradigm can or should be used to assess any issue." This would suggest that there may be significant dangers in attempting to cling dogmatically to any single paradigm when making methodological decisions. In conducting his craft, the researcher must engage his subject in a mode which both reflects the nature of the phenomenon itself and his own values and interests. The philosophical positions indicated in figure 4.1 thus inform, but do not determine, the selection of an appropriate research methodology. It is important then for a researcher in 
marketing to understand the relative strengths and weaknesses associated with a particular mode of engagement with the research subject (Brownlie et al., 1994). In this way it is possible to set out the parameters within which the theory generated can be regarded as 'true' and pertinent to some notion of 'reality'. This is the goal to which the following section is orientated.

\subsection{Researching Morality in Marketing - Methodological Implications}

Although a great deal of time and effort has gone into evaluating the validity and reliability of different research methods, the value of any method essentially derives from its potential to generate rigorous theory and useful knowledge. Therefore, this section shall concentrate solely on those aspects of engagement which are particular to the moral aspects of marketing activity, and which are likely to have important methodological and epistemological implications. Broadly, these can be considered under the following headings: status of the field, sampling, measurement, frame of reference, bias, and context.

\subsubsection{Status of the Field}

Research concerning the social and moral dimensions of marketing has been criticised for attempting to develop normative prescriptions before adequately understanding the research field itself (Hunt and Vitell, 1986). Essentially, with rapidly changing social and ethical demands and the appearance of new business opportunities along moral dimensions, the subject area is still attempting to progress out of the exploratory stage of an immature field of research. As a 
consequence, one of the criticisms most commonly levelled at such research is that the concepts being applied by researchers are not uniformly defined and understood, whether by other researchers or by research subjects (Sethi, 1974; Aldag and Bartol, 1978; Bain, 1995). With a lack of clarity of concepts still evident in the field, there would appear to be certain dangers implicit in the construction of survey instruments, and even in the collation of existing published company data, since these assume a certain unanimity of meaning (Gray et al., 1995b).

In this scenario, where the research field is regarded to be relatively underdeveloped, it can be argued that the principal purpose of empirical research should be theory building or theory generating (Glaser and Strauss, 1967). Positivist methodologies however favour the testing and verification of theory, and therefore may be inappropriate - at least when applied in isolation from other methodological approaches - for the questions to which this study is oriented. Accordingly, the case study may be a more appropriate mode of engagement in exploratory research of this kind (Bonoma, 1985; Smith, 1991). Essentially, case study enquiry allows the researcher to investigate a phenomenon within its real-life context and to utilise multiple sources of evidence in order to build up a more informed and holistic understanding of the research field (Yin, 1989; Smith, 1991).

\subsubsection{Sampling}

Unlike traditional organization functions such as production, finance, marketing, personnel etc. which are generally located primarily within a single department, there is only very rarely a department or even an individual responsible for 
corporate morality. Whilst environment managers are slowly beginning to become slightly more prevalent in the corporate landscape, even they are likely to be attached to any one of the many conventional organizational departments (Fineman, 1997). There is as a consequence frequently no obvious target for the researcher to identify for the purpose of data collection. The marketing department may actually have little knowledge, expertise or experience of many of those ethical issues which affect its operations; hence, product safety may be controlled through the quality department, environmental impacts through production, and so on. Drumwright (1994) for instance reveals how one of the key findings of her study - the identification of environmental champions or "policy entrepreneurs" as major protagonists in internal green procurement may have been completely missed if survey instruments rather than interviewbased case studies had been used as her main form of data collection since these individuals were frequently located outside of the conventional buying function.

Differences in individual attitudes, professional roles and hierarchical position are all likely to influence the way in which corporate moralities are perceived and understood by organization members. A cultural analysis of the moral realm of the organization therefore has to attend to the possibility of disunity of moral meaning rather than accepting any single viewpoint. It is possible then to take account of sampling problems such as these, but only if the researcher is willing to engage the research subject directly and build up some kind of relationship so that both deeper and broader access is secured. Indeed, with the focus in the current study on organization culture as the bridge between abstract moral principles and everyday organizational life, then research must account for the multiplicity of layers and the highly interactive dynamics of the cultural milieu 
(Pettigrew, 1979; Schein, 1992). The necessity for multiple respondents across organizational divisions and hierarchies in this sense is evident.

\subsubsection{Measurement}

Positivist methodologies place a strong emphasis on measurable data. However, there exist substantial problems for researchers in constructing appropriate instruments to assess aspects of morality in marketing. In particular, attempts to apply a measure of morality, ethics, social responsibility, or 'greenness' have met with only limited success, and this has rendered the use of quantitative approaches extremely problematic. Indeed, some measures of social responsibility (Wood et al., 1986; Hunt et al., 1990) and ethical values (Hunt et al., 1989) appear especially unconvincing. For example, Wood et al. (1986) estimate social responsibility based on a two item index which merely inquires of individual marketing executives' agreement or disagreement with the following two statements: (i) I often place my duty to society above my duty to my company; and (ii) I often place my duty to my company above my duty to society. Even with later extensions to this 'model' (Hunt et al., 1990) to incorporate two more behavioural statements (replacing 'often' with 'sometimes') and four attitudinal statements, the research still lacks conviction as an exercise in generating or testing theory.

From a managerial point of view, however, one of the most obviously appealing aspects of the debate surrounding morality in marketing from an empirical point of view is the possible relationship between 'socially responsible' or 'ethical' behaviour and the firm's economic performance or market share. The assertion that 'good ethics is good business' is at the heart of a variety of tracts written 
within this area (e.g. Abratt and Sacks, 1989; Davis (J.J.), 1994); the inherent difficulties in defining and measuring morality variables however have severely limited the application of quantitative techniques in the testing of this relationship. Consequently, empirical research has yet to deliver any clear evidence to support this argument, and the proposed relationship remains equivocal (Aldag and Bartol, 1978; Owen and Scherer, 1993).

As a result of some of these problems in measuring morality, other researchers have attempted to establish 'more objective' proxy measures for social responsibility based on components of social performance, e.g. pollution (Dooley and Lerner, 1994), or of social responsiveness, e.g. management structures (Greening and Gray, 1994), using combinations of questionnaire responses and published data. These have then formed the basis of quantitative analyses which have generated potentially more fruitful and compelling results. One major drawback however of using social performance/responsiveness measures is that the insights subsequently gleaned into marketing ethics are of the utilitarian type (based on outcomes) at the expense of deontological considerations (based on motivations), although it is the latter which are considered to be vital for progress in marketing ethics research and practice (Hunt and Vitell, 1986), and are clearly central to any understanding of moral meaning.

The question that remains then is whether the relative weakness of the existing attempts at quantification of morality variables is simply a reflection of the relative 'youth' of the field. In this case, it is arguable that measurement scales will follow an evolutionary path leading to increasing sophistication and 
applicability. Or, is morality one of the areas which Bonoma (1985) identifies as of interest to researchers but which simply defy counting approaches? Certainly, at this early stage in theory development, it is difficult to believe that the complexity of morally relevant marketing phenomena is likely to allow for adequate quantification of many of the variables of interest to the current study; moreover features such as those considered below might limit the scope of what can or should even be measured.

\subsubsection{Frame of Reference}

The fourth issue, although related to those above, is perhaps then even harder to counter: there is the danger that in conducting empirical research on a moral dimension, the researcher will automatically impose their own normative frame of reference onto their subject. Positivist assumptions in particular tend to imply that ethics are objective, knowable facts uncontaminated by subjective and relativist interpretations. Moreover, it is assumed that such facts are value-free and therefore independent from the researcher. From an interpretive perspective, the positivist researcher will therefore import their own ideas about what is meant by 'responsible, 'ethical' or 'altruistic' etc. into the research field and is therefore unlikely to understand and take into account: a) how these notions are actually used, interpreted and understood in the corporate setting; and b) the role of their own values in framing and influencing the research. This might effectively impose a normative straightjacket on respondents, denying their opinions proper voice and truth claims.

Morality, ethics, social responsibility - these are highly complex notions which can be interpreted in wildly different ways. Shared moral meanings and 
relationships must in some way hold together any community that can be characterised in terms of its culture (Anthony, 1994). However, these shared meanings may be diverse, shifting or even fragmented into multiple interpretations (Martin, 1992). Fineman $(1996,1997)$ for example reveals that the moral meaning of the environment within the organization is open to interpretation and is, to a larger extent, negotiable. Internal and external communications therefore constitute excellent data regarding how notions of morality are expressed and instituted, but in order to be deciphered, must be examined in the context of the organizational processes that proceed the production of the text (Phillips and Brown, 1993). Language too is in itself an important conduit of morality, and therefore moral discourse provides a fertile source of data for empirical work in studying morality in marketing (Bird and Waters, 1989). Survey instruments however have only limited potential to grasp the complexities and nuances of moral discourse because they tend to be devoid of the type of cultural meaning which is required to 'read' it (Brigley, 1995).

The methodological implications of these organizational characteristics are significant. The a priori construction of morality variables prevents the quantitative researcher from accounting for, or even revealing, differing organizational conceptions and understandings of 'ethical' or 'socially responsible' activity. Indeed, 'the spectrum of organizational life is filtered through the researcher's pre-set categories" (Evered and Louis, 1981). Whilst there is no reason to suggest that case study methods will necessarily avoid these problems, there is at least scope for the interpretive researcher to be reflexive and question their own normative assumptions and terminologies. Thus it is possible for the researcher to 'bracket' their own beliefs and to induce culturally specific 
moral meanings from the data (Miles and Huberman, 1994).

\subsubsection{Bias}

One of the main problems for investigators that utilise methods which require direct responses from research subjects concerning morality is the possibility of encountering social desirability bias. When asked the question "is your company green?" or "is your company ethical?" there are few executives that would tick the "no" box. Whilst interview methods are also affected, it is survey research in particular which has been criticised on this account due to its lack of flexibility in the face of such bias (Aldag and Bartol, 1978). Moreover, evidence suggests that self-reports by executives in surveys are subject to considerable inaccuracy and bias (Mathews and Diamantopoulos, 1995).

Obviously, the existence of such bias will impose significant limits on the scope and method of empirical work investigating morality in marketing. Whilst methods exist whereby the researcher can, to a certain extent, account for bias in quantitative work, the use of multiple data sources or 'triangulation' may be a more successful approach. This is evident in the survey work of Dooley and Lerner (1994) and Greening and Gray (1994) among others who have attempted to incorporate archival data in order to 'check' executives' survey responses. In case study work from a more radical structuralist perspective, Nichols (1969) has provided a valuable insight into how managers attach meanings to notions of social responsibility. He not only uses interviews to check managers' responses to a fixed choice 'moral statement' questionnaire, but also utilises them to explore the reasons underlying the managers' responses. Crucially, he found that 
a majority of respondents could not perceive ideological difference between 'practical' enlightened self interest positions and 'ethical' social responsibility positions, and indeed, interviewees tended to explain their questionnaire responses of the latter type in terms of arguments of the former. Thus, "we were often fascinated by the way in which businessmen were able to hold two, for us, contradictory views without themselves perceiving any conflict between them," (1969:250) - an important discovery in itself. Essentially then, if it is accepted that executives know with little precision factual information concerning their jobs (Mathews and Diamantopoulos, 1995), nor even what they themselves actually do on a day to day level (Mintzberg, 1989), those interpretive approaches which are based on meanings rather than functionalist approaches based on facts may be regarded as epistemologically more appropriate to questions where desirability bias is most likely. Crucially in this respect, the focus in the current study on green marketing means that morality need not be empirically investigated directly - as in "is green marketing in your company ethical?" - but in an indirect, even elliptical manner - as in "describe green marketing in you company". Using this approach, moral meaning can be inferred, thus reducing the possibility of desirability bias.

\subsubsection{Context}

For the marketing researcher then, it is important that morality should be understood within the exigencies of the prevailing organization culture and climate. Essentially, morality is neither objective nor factual, but rather is given meaning in a highly contextual and subjective sense (Jackall, 1988). Harvey et al. (1984) thus argue for the need for corporate social responsibility research to be conducted such that it can take account of both contingency and internal and 
external context. Jackall (1988:5) too asserts that, "only an understanding of how men and women in business actually experience their work enables one to grasp its moral salience to them." Whilst some quantitative approaches have been attempted for the study of ethics and cultures (see for example, Vitell et al., 1993; Diacon and Ennew, 1996a), this approach has been widely criticised with respect to its suitability for studying deep cultural forms (Alvesson and Berg, 1992). Indeed, recognising that the cultural milieu can effect moral deliberation and moral choices does not imply that corporate culture exists as a concrete and objectively definable phenomenon. Rather, it can be considered as a product of organization members' intersubjective experience; culture only exists to the extent that it is meaningful to organizational actors in terms of how they attempt to construct relevant and coherent frames of reference (Geertz, 1973).

Contextual sensitivity is essential then for much of what the marketing scholar might research along moral dimensions; as such there is considerably more potential for knowledge generation evident in case study "inquiry from the inside" (Evered and Louis, 1981) research than there is in more positivistic approaches (Bonoma, 1991). In interpretive accounts based on qualitative data there is the opportunity to utilise a strategy characterised by, "deep attentiveness, of emphatic understanding (verstehen), and of suspending or 'bracketing' preconceptions about the topic under discussion," (Miles and Huberman, 1994:6). Essentially, the researcher that does not interact directly with his subject is in danger of de-contextualising relevant moral issues to the extent that essential layers of meaning are inevitably stripped away and lost. Thus, "many issues of interest to marketers can not be studied outside the context in which they naturally occur," (Bonoma, 1985:202); morality, it can be argued, is one 
such issue.

This discussion goes some way towards revealing that certain features which are intrinsic to the business ethics field render the application of functionalist approaches problematic in terms of their scope of application and facility for theory building. In particular, research methodologies which define moral terms, concepts, choices and scenarios prior to data collection may, in some key aspects, be inferior to those which develop them inductively from the research process. In the study of morality in marketing then, understanding meaning must precede measurement - and this requires a degree of cultural sensitivity not as yet evident in much of the current use of positivist approaches. So how is this cultural sensitivity and understanding to be acquired?

\subsection{Researching Organizations as Cultures}

It has been suggested during the discussion above that the interpretive paradigm would seem to offer substantial epistemological potential in the study of morality in marketing. This study then takes a social constructionist perspective, i.e. it is based on the proposition that organizational reality can be regarded as a dynamic social construction which is formed through the interaction of individual members who in turn are affected by their membership of the social organization thus created (Berger and Luckmann, 1966). In this scenario, organization members create, absorb and interpret the systems of shared moral meanings which comprise their cultural reality. The key to seeing, understanding and knowing cultural reality within the social constructionist tradition lies in unravelling the meaning that organizational actors attach to the everyday reality around them. Such research then focuses on "the realms of meaning and 
interpretation," (Smircich, 1985).

The concept of culture as a mode of engagement is not however without its critics. In particular, there are the obvious problems associated with conceiving culture as everything - "the kitchen sink syndrome" as Martin (1992) refers to it. Essentially though, the cultural understanding of organizations focuses attention on the symbolic realm, and on how various cultural texts such as language, stories, artifacts, behaviours etc. create and transfer meaning through symbolic means (Pettigrew, 1979). The position adopted here is that in addition to the symbolic realm, a cultural analysis also focuses attention on the political milieu of the organization. As Phillips and Brown (1993) argue, the symbolic elements of culture cannot be interpreted properly without an understanding of the power structures underlying them. Some comment on these two aspects of culture is therefore warranted before proceeding further.

\subsubsection{Symbolic-Cultural Analysis}

Marketing trades in symbols and signification (Levitt, 1972). As such the marketing culture can be regarded as one saturated with images and symbols (Christensen, 1995). Similarly, Turner (1992:58) regards all organizational research as stepping into a "vortex of symbols and symbol transformation." Therefore if it is to be possible to understand the organization from a cultural perspective, it is essential to unravel the symbolic interaction at work (Turner, 1992); in order to study social significance, one must first study the signs in which it is embodied and transmitted (Barley, 1983). In making a cultural analysis of the organization it is imperative then that the researcher acquires qualitative data that are, "symbolic, contextually embedded, cryptic, and 
reflexive," (Van Maanen, 1979a:521). Barley (1983) suggests that the most appropriate mode of inquiry in this instance is semiotics, which he defines as, "the study of signs or systems of signs....semiotics concerns the principles by which signification occurs," (p.394). However, Turner (1992) among others criticises this position for asserting that the symbolic discourse itself represents the social reality, that in effect, "the medium is the message", such that we must, "transform inquiry about the 'reality' of 'things' into an exploration of codes," (p.51). Turner argues that, in fact, symbols do not simply emerge from human interaction loaded with inherent meaning, but that they are invented through a creative act of image making by individuals and their organizations. The logical deconstruction of the symbolic discourse can only provide a partial picture of organizational reality because the process of signification does not follow any standardised rules, and presents us with multiple realities and a multiplicity of meanings. Turner thus emphasises the need to "reject the reduction of the institution to a mere symbolic structure," (p.54), and "turn our attention to the processes by which reality emerges in the continuously created culture of an organization," (p.58).

The approach adopted here then rests on the assumption that organizational morality does not exist solely in the realm of symbolic discourse, but that it is socially constructed through systems of shared meaning. Therefore, the mode of engagement adopted has been one which allows for the understanding of the ways in which notions of morality are expressed, interpreted and understood during green marketing campaigns. This moves beyond an analysis of the moral meaning embedded in symbolic texts and into an exploration of the ways in which organizational members seek to interpret these texts and their context in 
order to construct moral frames of reference for their everyday experience in (and of) the organization. The intention then is to provide "thick description" (Geertz, 1973), and elucidate second-order concepts, or "interpretations of interpretations" (Van Maanen, 1979b). In order to achieve this the investigator has adopted an interpretive mode of engagement, adopted the role of learner (Smircich, 1983b) and has attempted through observation, interview and documentary examination to unravel the patterns of meaning that underlie social cognition and interaction (Morgan, 1983b). This has involved not only symbolic understanding, but also insight into the political realm of the organization.

\subsubsection{Political-Cultural Analysis}

Whilst Phillips and Brown (1993) also argue that in making a cultural analysis of the organization it is important to understand the symbolic processes through which socially constructed systems of meaning emerge, they argue that this should be supplemented with a political analysis of the organization. The symbolic conception of culture therefore does not generally take our understanding of the process of moral signification far enough. Thus (p.1551):

In understanding culture as symbolic, but failing to emphasise the differing social resources of different actors, symbolic cultural theorists have failed to include a central aspect of the process by which culture is created and sustained. Although everyone participates in the creation and maintenance of culture, not everyone participates equally.

In this sense, marketing practice can be understood better as a highly political process undertaken by individuals and groups guarding sectional interests and 
agendas within the marketing landscape rather than as a highly rational, scientific process leading to market-dictated corporate goals (Brown and Ennew, 1995). The central research issue then becomes the process by which certain individuals or groups of individuals succeed in developing a collective frame of reference that is perceived as more legitimate or acceptable than that of others. Following Phillips and Brown (1993), culture then shall be viewed not simply as symbolic, but as structural-symbolic. As a consequence methodological choices must reflect two goals: the reading of the symbolic discourse, the organizational texts that deal with issues of morality in relation to the environment; and a critical understanding of the systems of power which shape how those texts are created and interpreted both inside and outside of the organization. These are the goals about which the research strategy has been constructed.

\subsection{Research Strategy and Method: A Grounded Theory Approach}

The basic research objectives formulated following the initial review of extant literature in the formal research field of marketing and morality (chapter 2) and the substantive area of green marketing (chapter 3) were set out at the end of the previous chapter (section 3.5). These were as follows:

1. to describe the green marketing process in detail;

2. to articulate the role of morality in that process;

3. to set out the cultural dynamics through which morality is given (or denied) expression and meaning in green marketing.

It has been shown that these questions have received little attention to date and that the general field of study remains at present empirically under-developed 
and theoretically shallow. This should therefore be regarded as an exploratory study, and one which attempts to move beyond description to develop original and systematic theory 'grounded', or 'discovered' in data (Glaser and Strauss, 1967; Turner, 1983; Eisenhardt, 1989; Strauss and Corbin, 1990). This means that "research is begun as close as possible to the ideal of no theory under consideration and no hypotheses to test," (Eisenhardt, 1989:536). Hypotheses, concepts, and theory constructs were thus not considered a priori, but were allowed to emerge from the field data as part of the research process (Glaser and Strauss, 1967). The precise mechanics of this process, and justification for the methodological decisions taken is set out in the sub-sections following. These cover the selection of the case study methodology, case sampling, data collection, data analysis, theory development, and validation.

\subsubsection{The Case Study Research Strategy}

Case studies are widely regarded as an important grounded theory approach (Glaser and Strauss, 1967; Turner, 1983; Eisenhardt, 1989). Indeed, where research is principally exploratory in nature, where the field is complex and sensitive, and where the principle purpose of the research is theory building or theory generating, it is case study research which is regarded to be the most appropriate mode of engagement (Bonoma, 1985; Yin, 1989; Smith, 1991). Yin (1989:23) defines the case study as, "an empirical enquiry that investigates a contemporary phenomenon within its real-life context; when the boundaries between phenomenon and context are not clearly evident; and in which multiple sources of evidence are used." Therefore it allows for multiple levels of analysis and can draw out the interdependent qualities of the cultural milieu of the organization (Pettigrew, 1979). Others have argued persuasively for research in 
marketing (Bonoma, 1985), business ethics (Harvey et al., 1984; Jackall, 1988; Drumwright, 1994; Brigley, 1995) and green business (Gladwin, 1993) to focus on case study 'inquiry from the inside' (Evered and Louis, 1981) in order to reveal the impact of cultural, structural and contingency factors on the moral reflection and decisions of organizational members.

\section{Case Selection}

The main basic decision in case study analysis is whether to develop a singlecase or multiple-case framework. This is a matter of considerable dispute within the interpretive tradition of organizational research (Dyer and Wilkins, 1991; Eisenhardt, 1991), but it is apparent that there is no absolute universal superiority of either strategy. Indeed, it is evident that within a grounded theory approach the choice should depend on the relative contribution of case study data to the strength of the resulting theory. In the current study, the need to get a deep, rich, and holistic view of the phenomenon under study and its context meant that intense and in-depth contact with fewer cases was regarded as superior to relatively superficial contact with a large number of cases ${ }^{21}$. Hence, substantial cross-case comparisons were not deemed as important as within-case comparisons for the generation of good theory (Glaser and Strauss, 1967). Nonetheless, the comparative case study methodology has recently produced some excellent 'grounded' theory based on qualitative research in the study of morality in marketing (e.g. Drumwright, 1994; 1996), business (e.g. Jackall, 1988) and green business (e.g. Fineman, 1996) and can be seen to provide

\footnotetext{
${ }^{21}$ Pragmatic considerations of time, resource and access limitations certainly came into play here. The scope of this study precluded the use of a larger number of individual case studies given the depth of analysis required.
} 
unique and important insights into the moral realm of the organization. It was therefore decided to adopt a comparative case methodology, selecting relatively few but as far as possible 'different' organizations in terms of their likely orientation towards the environment. This was considered to allow for considerable depth of insight, but over a range of quite significantly different contexts.

So how were particular cases selected? In contrast to the situation where the intention of the case study is to test theory, in the generation of new theory there is no requirement, nor even desire, to achieve random selection in case sampling (Eisenhardt, 1989). This study has therefore relied on theoretical sampling (Glaser and Strauss, 1967), such that cases were chosen because they were likely to replicate or extend emergent theory (Eisenhardt, 1989). The sampling framework adopted was very simple in structure. The starting point was the 'deep' vs 'shallow' perspectives on green marketing set out in the previous chapter. Since these articulated very different roles for morality in green marketing, it was decided that a full picture of green marketing moralities would have to attempt to describe the practical realities of both. There were though two major problems in this respect: first, it was problematic to determine a priori whether particular organizations would exhibit a 'shallow' or a 'deep' green marketing paradigm; and second, the existence of the latter, certainly in its purest form, was clearly questionable in the current economic environment. A more straightforward approach then was to focus on certain uncontroversial and relatively 'objective' elements of the 'shallow' vs 'deep' distinction and to select organizations exhibiting these. Given the emphasis in the 'deep green' paradigm on elements such as alternative 'ethical' management paradigms, the basic 
distinction chosen was that between organizations which clearly gave primacy to conventional economic goals, and those that were very clearly founded expressly in order to pursue particular social or environmental goals. We refer to these as conventional companies and social mission companies respectively. Such a categorisation is not meant to imply that conventional companies could not have social goals or that social mission companies could not have economic goals, but rather that the overall emphasis in corporate identity, branding, promotional texts, and other company documentation as well as published articles and corporate histories clearly indicated one or the other as the more prominent.

As the protracted process of negotiating access continued, and as initial fieldwork was conducted, one of the strengths of qualitative research, serendipity (see Miles and Huberman, 1994), began to have a profound effect on the sampling strategy. One of the case companies was found to be part of the WWF 1995 Plus Group, a coalition between the WWF and a number of UK businesses aimed at improving world-wide forestry practices. Clearly this was a social mission organization of sorts since its aims were principally environmental; but as a collaborative organization between a whole range of independent organizations - conventional, social mission, and not-for-profit - it was also very different from the original classification. It seemed plausible that this collaboration between organizations with diverse goals, orientations, and management paradigms might describe a very different organizational morality. Moreover, such "green alliances" were found to have been identified as a potentially important new strategic approach to green marketing, but had not been subjected to extensive empirical research (Mendleson and Polonsky, 1995; Wasik, 1996; Hartman and Stafford, 1997). It was thus decided to incorporate 
the phenomenon of business-NGO collaboration as a separate category of organization in addition to conventional and social mission companies. This provided the study with a three-section sampling frame.

With within-case depth more important than across-case width, the intention of the study has been to sample a small number of representative organizations from each of the three categories. Given though the degree of access required in order to conduct such an in-depth qualitative investigation, the sampling procedure can not be disassociated from the pragmatic considerations of acquiring and negotiating access with potential case organizations. In all some 85-90 companies were approached concerning the possibility of including them in the research. Most of these initial contacts were 'cold' in the sense that the researcher had had no previous contact with the company, nor the benefit of any personal recommendation with which to begin to negotiate access. In a number of cases no response at all was received as a result of this initial contact, and therefore follow-up letters were sent. For those that did respond positively, a number of letters and telephone calls were frequently required in order to gain successful access. Even then, the extent of access allowed to the researcher often had to be continually re-negotiated as the fieldwork proceeded and further data were required. The resulting sample of cases is shown in figure 4.2 below.

Clearly there are some limitations to the case sampling strategy outlined here, particularly in the sense that all cases were to some extent self-selected (although the majority of actual respondents were not). This however has been incorporated into the data analysis. Indeed, Jackall (1988) asserts that a great deal can be learnt concerning organizational morality from problems 
encountered with organizing field access and organizational response. Accordingly, the process of arranging access, in the sense that it can be regarded as an interaction between the organization and an external stakeholder ${ }^{22}$, has been accorded status as primary "stimulated" data (Salancik, 1979:638).

\section{Figure 4.2: Case Study Sample}

\begin{tabular}{|c|c|c|}
\hline $\begin{array}{l}\text { Conventional } \\
\text { Companies }\end{array}$ & Social Mission Companies & $\begin{array}{l}\text { Business-NGO } \\
\text { Collaboration }\end{array}$ \\
\hline $\begin{array}{l}\text { - Boots the } \\
\text { Chemist } \\
\text { - ITT Flygt }\end{array}$ & $\begin{array}{l}\text { - Beauty Without Cruelty } \\
\text { Bioregional Charcoal } \\
\text { Company } \\
\text { - Co-operative Wholesale } \\
\text { Society } \\
\text { - Ecology Building Society }\end{array}$ & $\begin{array}{l}\text { WWF } 1995 \text { Plus Group } \\
\text { (data collected from } \\
\text { sample of member } \\
\text { organizations) }\end{array}$ \\
\hline
\end{tabular}

\subsubsection{Data Collection}

Data collected were primarily, but not exclusively, qualitative in nature. Miles and Huberman (1994:10) identify a number of key strengths of qualitative data that were instrumental in the development of the research strategy outlined here. Most notable of these are that data exhibit: first, a local groundedness (data are

\footnotetext{
${ }^{22}$ When asked about relationships and communications with external publics, a number of interviewees indicated the current interview with the author as an example.
} 
contextually embedded), and so there is a high possibility of uncovering "latent, underlying or non-obvious issues"; second, a richness and holism which is essential in providing "thick description"; third, an element of process rather than simply structure; and fourth, an emphasis on lived experience which enables the researcher to locate meanings and connect them to the social world.

Data were collected and recorded in a number of ways. The main focus of the data collection was a series of 60 face-to-face, semi-structured interviews. Usually these were conducted with single respondents, although on two occasions two interviewees were present. In addition, two small focus group interviews were conducted with shop-floor respondents (with 4 and 3 members) in order to make best use of the access provided, to avoid intimidating interviewees, and to explore deeper interpretations brought on by interviewee interaction. Interviews were conducted by the investigator at the respondent's place of work and the majority were recorded on audio tape. These were then transcribed as soon as possible after the interview had taken place in order to permit immediate grounded theory analysis. Where recording was not possible or practical, extensive notes were made and subsequently typed up. Where faceto-face interviews were not possible in three cases, telephone interviews were conducted. The duration of the face-to-face interviews varied considerably, from as little as 15-20 minutes in a few cases to well over two hours in others. The shorter ones tended to be with shop-floor employees who were not allowed to leave their posts whilst the interview took place. Much of this data was necessarily thin. The majority of interviews though were of approximately one hour duration, and this was also the overall average interview duration. 
In keeping with the exploratory nature of the research, the precise nature of the interview schedules used was continually adjusted in order to explore emergent themes. However, the important point to note is that interviewees were deliberately approached with an understanding that the focus of the study was "green marketing and management". No mention of morality was made on the investigator's part at the outset, and even as the interview progressed this would rarely be introduced unless at the interviewee's instigation ${ }^{23}$. The purpose of this was to investigate moral meaning in a more indirect way, such as to reduce the level of desirability bias and to try and gain more 'naturalistic' insights. It was hoped that respondents' everyday frames of references would emerge from the discussion rather than those imposed by the researcher.

Substantial amounts of other data were also collected. Evidence emerging from events, such as formal and informal interaction and observation, and from internal communications, such as documents and reports were recorded in comprehensive field notes. In a few instances, the case companies supplied video tapes of company conferences and training videos. Relevant external communications were recorded either as physical artifacts (for products, packaging, and print advertisements), or on videotape (for television commercials). Again, these were analysed as soon as possible in order to expand the field notes and develop the emergent grounded theory. A field diary was maintained throughout the study in order to record ideas and inspirations, and in which to set out any immediate and/or on-site analysis. In all over 80 hours of data were recorded over a 16 month period.

\footnotetext{
${ }^{23}$ Copies of the research proposals sent to prospective case organizations and model interview schedules are provided in appendix $\mathrm{A}$.
} 
Most of the data collected were therefore in the form of words, although these were not necessarily regarded as 'natural' or 'true' data, but rather as interpretations based on the investigator's own perceptions, and implicit concepts (Miles and Huberman, 1994), and as texts shaped by the nature and language of the investigator's questions (Salancik, 1979). There are of course dangers inherent in assimilating a large volume of such data and so, following Van Maanen (1979b), care was taken as far as possible on two counts: first, to distinguish between informants' first-order conception of what was going on (the 'facts'), and the investigator's second-order conceptions of what was going on (the 'theories'); and second, to distinguish between first-order concepts comprising of "operational data" (spontaneous activity), and those comprising of "presentational data", defined as "appearances that informants strive to maintain (or enhance) in the eyes of the fieldworker, outsiders and strangers in general, work colleagues, close and intimate associates, and to varying degrees, themselves," (1979: 542). In the first instance, the intention was to elicit the meaning behind the data, and in the second, it was to reveal "simulated" or "seduced" reality (Turner, 1992). Data which could be regarded as "seduced" reality was not however discarded as worthless. In recognising such presentational data as symbolic, it was possible to further enhance the richness of the cultural analysis. Indeed, a recognition and understanding of the act of image-making is as central to the reading of internal organizational reality as it is to the reading of deliberate external texts (Brown, 1994). Thus as the multiplicity of cultural and symbolic layers emerged, so the process of analysis became more complex, and the degree of mediation increased. 


\section{Data Sampling}

In generating grounded theory, within-case data is sampled theoretically, such that, "the analyst jointly collects, codes, and analyses his (sic) data and decides what data to collect next and where to find them, in order to develop his (sic) theory as it emerges," (Glaser and Strauss, 1967:45). The process then was a lengthy and highly iterative one. Having begun preliminary fieldwork in September 1995, data collection was finally completed in December 1996. The interview profile is shown below in figure 4.3.

As far as possible, sampling was intended to elicit an in-depth understanding of the green marketing process associated with the case organizations, and this necessitated sampling across organizational hierarchies and functions. In many instances the most important insights into this process came from outside of the marketing function itself, and therefore a large proportion of interview respondents were actually non-marketing professionals. This was not in itself seen to be a weakness of the study since our unit of analysis was very much the marketing process rather than marketing managers themselves.

The sampling methodology became more directed as the study progressed. The flexible but purposeful strategy of open sampling at the outset was superseded by a more focused and deliberate process as the theoretical categories, concepts, and relationships became more distinct (Strauss and Corbin, 1990). Data sampling was thus led by the emergent theory. However, limitations were imposed by the level of access granted by the case organizations. Moreover, in order to prevent "death by data asphyxiation" (Pettigrew, cited by Eisenhardt, 1989:540), as far as possible data was generally only sampled to the extent that it 

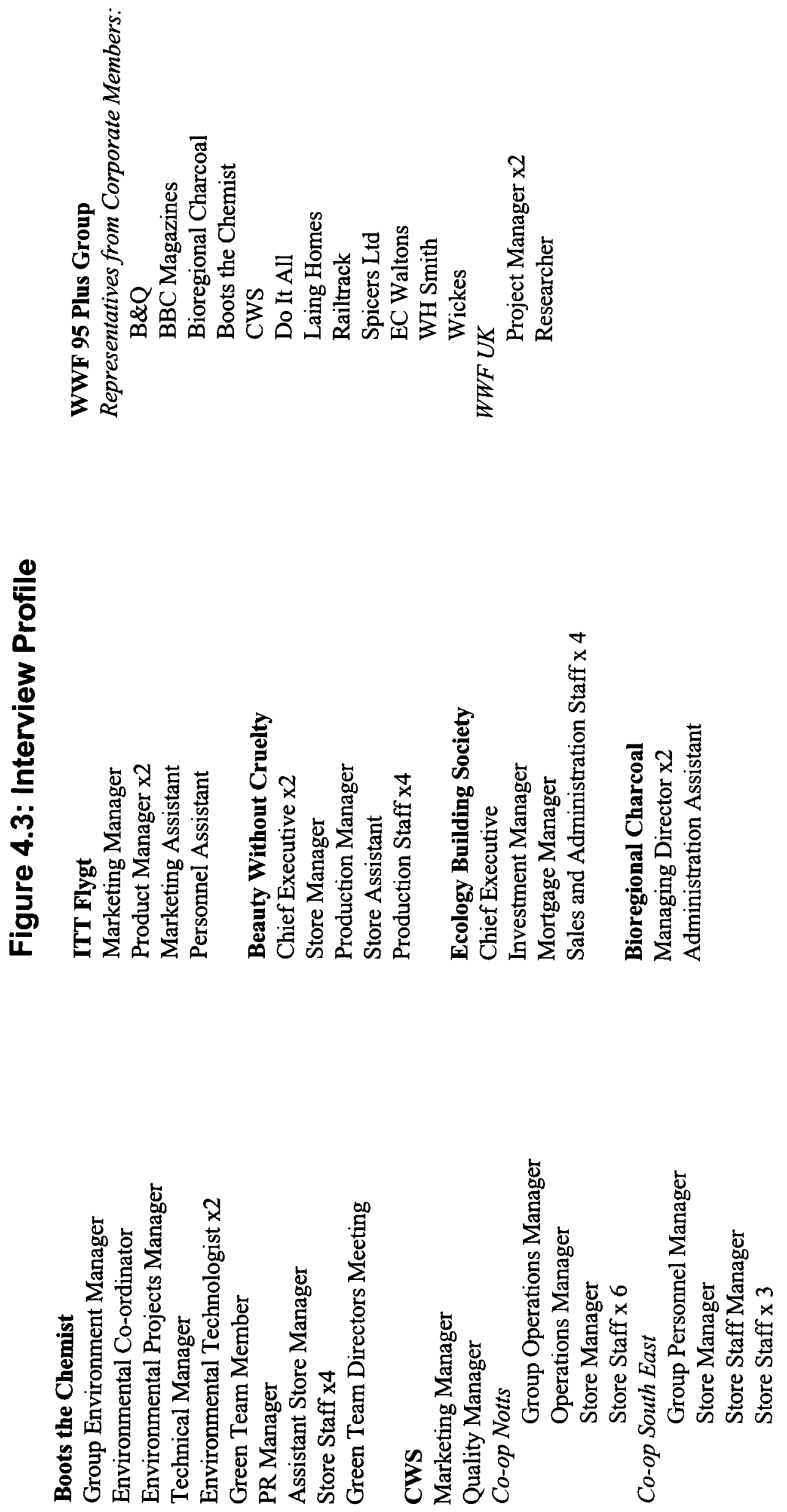
was likely to have had any bearing on emergent categories, dimensions, and relationships, i.e. up to the point of theoretical saturation (Glaser and Strauss, 1967). However, the adoption of this strategy was not considered to demand the didactic adherence to an overly systematic sampling framework, such as that advocated by Eisenhardt (1989) and Strauss and Corbin (1990), for it was recognised that there should always be sufficient flexibility in sampling to reveal the unexpected, the unusual, the interesting, and other serendipitous findings.

\subsubsection{Data Analysis and Theory Development}

The generation of theory in this study has followed a grounded theory approach, i.e. the discovery of theory from data. This is primarily due to two factors: first, the nature of the phenomenon under investigation, in that there is at present little rigorous theory in the research area; and second, Glaser and Strauss' (1967) argument that 'better' social theory can be generated inductively from data rather than from abstract deduction and hypothesis-testing. Thus, the principal methodological aims were in "discovering theory as a process," (Glaser and Strauss, 1967:9), and in removing the "strictures that favour validation rather than usefulness," (Weick, 1989:516).

Theory construction within the interpretive tradition has been variously described as "semi-tacit craft skill," (Turner, 1983:334), "disciplined imagination," (Weick, 1989:516), "the telling of good stories," (Dyer and Wilkins, 1991:613), and "a systematic set of procedures," (Strauss and Corbin, 1990:24). One of the key principles then of qualitative research is that theory building combines rational elements with intuitive, or imaginative elements. These can be regarded as different points on a continuum that runs between the 
rational and intuitive approaches to theory building. To a greater extent, the position of a particular theory on this continuum owes more to the skills and specialities of the researcher than to any other factors. The intention here then has been to craft a powerful and evocative story depicting the moral experience of individuals who have been involved in the development of green marketing programmes. The relatively formal analytic procedures advocated by Glaser and Strauss (1967), Eisenhardt (1989), Strauss and Corbin (1990), and Miles and Huberman (1994) have been used as a basis to develop a slightly more informal "emergent" analytical strategy that suited better the research field, the data collected and the skills of the data analyst (Mintzberg, 1979). The main elements of this were relatively 'rational' coding and memoing, and rather more 'intuitive' elements of thick description and thought trials. These two elements are considered next.

\section{Coding and Memoing}

Strauss and Corbin (1990:57) define coding as, "the operations by which data are broken down, conceptualized, and put back together in new ways. It is the central process by which theories are built from data." Deriving from the process described by Miles and Huberman (1994), the process of coding used here initially involved breaking down the data into chunks labelled with descriptive codes. This left a large number of various categories and dimensions, each of varying salience and interest. These were then analysed more fully and assigned meaning. Such second-order interpretations were labelled with a series of interpretive codes. As these concepts emerged, and filled the various dimensions revealed earlier, so it was possible to infer relationships between them and to describe patterns. These were then used to develop theory constructs and 
storylines.

However, contrary to the above description, it should be noted that this process of coding was carried out iteratively rather than linearly, and frequently informally rather than formally. Codes, concepts, relationships and constructs were constantly reworked and reconceptualised in order to explore emergent themes and stories. Coding thus switched continually between the inductive and the deductive - often utilising various ordered displays, spider diagrams and matrices - whilst ideas and interpretations were conceived and then compared again with existing data, and used to decide which data to collect next (Strauss and Corbin, 1990). The coding procedure was formalised through the generation of a substantial series of memos which were stored and analysed in a manual database.

\section{Thick Description and Thought Trials}

At the same time that this procedure of data codification and abstraction was carried out, it was also considered important not to sacrifice thick description and vivid stories to the generation of theory constructs. So although the principal aim of this work has been to develop new and analytically rigorous theory, a prerequisite for this has been the elaboration of contextually rich and interesting stories (Dyer and Wilkins, 1991). Therefore, the way that data was 'put back again' into the research monograph was considered to be equally as important as the way that it was broken down and conceptualised. Recognising this at the outset, and integrating it into the initial framework for generating theory, meant avoiding being left after the process of theory construction with a somewhat bald description which would then have had to be re-contextualised and 'thickened'. 
Therefore, following Turner (1983), two sets of files were generated during data analysis - one with which to develop levels of theoretical abstraction, and the second to follow interesting storylines suggested by the emerging theory. These two fields were constantly compared and integrated in order to form a nested set of emergent themes.

An important element in the development of grounded theory is that emerging concepts and theories are not objective as such but are mediated through the lens of the analyst's perceptions, prior experiences, and interests. Weick (1989) suggests that in order to construct good theory the analyst should use and explore his or her imaginative capabilities; the greatest danger, he argues, lies in pretending that these elements are not present at all. Dyer and Wilkins (1991) pursue a similar theme in criticising Eisenhardt's (1989) work, arguing that an over-emphasis on "sharply defined, measurable constructs," (1989:542) pushes the analyst towards a more functionalist conception of theory building based on testable propositions. This then incurs severe limitations on the analyst's ability to explore less obvious social dynamics and moral issues since deeper, nontestable or tentative insights will be eliminated from the process of theory generation.

In order to counter some of these problems, analysis in the current study utilised what Weick (1989) has referred to as multiple, independent thought trials. Since any event is open to multiple interpretations, it is important that theoretical insights are based not simply on one interpretation, or on the one first 'saturated' by data, but by many and diverse interpretations. In particular it was attempted to interpret the data through the lenses of the 'shallow' and the 'deep' green 
positions outlined in the previous chapter. This did not however extend as far as consciously attempting to conduct multiple paradigm research as advocated by Hassard (1993), but independence between trials was maximised as far as possible within the social constructionist perspective.

Having conducted multiple thought trials, either one of two situations was found to emerge: either the disparate interpretations could be related meaningfully at a higher level of abstraction; or alternatively, conflicting theories were developed. After Poole and Van de Ven (1989) the emergence of such "paradoxical" findings was not considered to be a problem per se, but rather as an opportunity to develop richer and more detailed theory. Three strategies were used to deal with paradox. Either second-order interpretation allowed for the explanation of the apparent paradox. Failing this, the paradox was used to discount one or other of the thought trials. Alternatively, another strategy involved accepting the paradox and using it constructively in the development of a more interesting story (see for example, Nichols, 1969). Either way, it was not considered appropriate to reject the occasional occurrence of inconsistencies and nonconfirmatory findings as insignificant (as is often recommended in quantitative analysis) whether this occurred between theories (Poole and Van de Ven, 1989) or even within them (Eisenhardt, 1989). Indeed, as Poole and Van de Ven (1989:563) argue, "theories are not statements of some ultimate 'truth' but rather are alternate cuts of a multifaceted reality." This obviously has important implications for how the grounded theory should be judged and validated. This is the subject of the final sub-section following. 


\subsubsection{Validation}

Two of the key principles of qualitative research are that it promotes theorising of an impressionistic rather than definitive nature, and that there are few statistical tests that can be invoked to suggest the validity of theory grounded in data. This makes the issue of validation a somewhat non-standard, often contentious, one. Nonetheless there are a number of tests of the quality of such theory and these are considered here under the headings of reliability, internal validity, and external validity.

\section{Reliability}

Testing for reliability is, in effect, a quality control measure (Miles and Huberman, 1994). Yin (1989:45) describes the objective of reliability tests as ensuring that, "if a later investigator followed exactly the same procedures as described by an earlier investigator and conducted the same case study all over again, the later investigator should arrive at the same findings and conclusions." Clearly, in a highly interpretive study such as this where the researcher has crafted and shaped his grounded theory, it is unlikely that another individual would reach exactly the same conclusions. Therefore, reliability is more practically informed when the research process can be traced stage by stage, and where its theoretical development can be followed and understood by a fellow researcher. In essence, it is important that an outsider can effectively view the material through the lens of the investigator. Accordingly, data collection and analysis in the current study has been relatively informal but carefully recorded, and has followed coding procedures well-documented as reliable in the qualitative analysis literature. 
A yet more conclusive assurance of reliability can be inferred by the extent to which 'within-method' triangulation has been exercised (Jick, 1979). Triangulation in this regard constitutes the process of exploring and utilising multiple sources of data, and this has been exercised where appropriate throughout the course of this study. Analysis has focused on evidence derived from interviews, observations, informal conversations, documents, products, marketing communications, and a limited amount of quantitative data. As suggested earlier, the purpose of this has not only been to ensure reliability and confirmatory findings; within-methods triangulation has also been used as a strategy to, "capture a more complete, holistic, and contextual portrayal," (Jick, 1979: 603).

\section{Internal Validity}

In contrast with Yin (1989) internal validity is not regarded here as inconsequential in an exploratory case study. However, the meaning of internal validity differs from that which is characteristic of problem-solving, hypothesistesting research. In the generation of grounded theory, issues such as credibility, authenticity, and plausibility are paramount in the testing of internal validity. Glaser and Strauss (1967) state that the requirements of a good grounded theory are that it must 'fit' the situation it is supposed to represent, and it must 'work' when put into use. To some extent then, the internal validity of this account should be assessed by the reader - does it make intuitive sense? is it logical? does it 'feel' right? Every attempt has been made here to ensure that the theoretical propositions put forward are internally consistent - that is that they fit together without contradiction and are mutually reinforcing. 
It is recognised however that the analyst's conception of this phenomena is not the only plausible one that could have been based on the data analysed. However, the highly iterative process in which data and emergent theory have been constantly compared, the independent thought trials conducted by the analyst, and the extensive assessment and evaluation of these rival explanations are all considered to contribute greatly to the high credibility of the results and conclusions presented in the following chapters. In order to fulfil the criteria of 'working', several sections of the resulting narrative have been presented back to the study's informants to confirm its correspondence with presumed realities and the plausibility of its explanations and interpretations.

\section{External Validity}

Problems of external validity are frequently cited as one of the main drawbacks of qualitative research, and of case study research in particular. Jick (1979) proposes that the problem of external validity in case study research can be best addressed through the use of multiple methodologies, or across-methods triangulation. This reflects a concern within the social sciences that research conclusions can reflect the methodology used rather than the phenomena itself (Jick, 1979; Morris and Wood, 1991). The use of multiple methods can therefore be used to increase confidence in the validity of conclusions. It is evident however that the link between epistemology and methodology is highly complex and relatively unproven (Morris and Wood, 1991). The proposition upon which the methodological decisions that are described here have been made is that the choice of methods should reflect the characteristics of the phenomena under study and also the conception of reality that is held by the researcher. Therefore, just as no single method is universally 'best', neither is any particular mix, nor 
the mixing of methods per se, universally 'better' than any single method alone. Though predominantly qualitative, the research reported here has though utilised some mixture of methods. In particular, textual analysis of interview transcripts and corporate documentation has in many instances been reinforced with quantitative content analyses.

The most pressing issue of external validity for the generation of theory from case studies is the problem of generalizability. Eisenhardt (1989) recommends the use of the case study as a powerful research tool, but feels that the problem of generalizability indicates a need to analyse between four and ten cases in order to produce convincing findings. Conversely Dyer and Wilkins (1991) argue convincingly for a single case design, thus enabling thick description and good storytelling. The ethnographic work of Van Maanen $(1973 ; 1991)$ provides an excellent example of how a single case setting can be used to explore phenomena at multiple levels - separate individuals and groups, different situations, different times, etc. - and these can be thought of as sets of microcases embedded within the main case. External validity can be inferred then to the extent that the theory generated describes and explains a large proportion of characters and situations within the study area, i.e. its 'thickness'. Indeed, it is proposed that the best test of external validity for research of this kind is what Dyer and Wilkins (1991) have termed the "aha experience". This is where rich and vivid description have revealed the dynamics of a situation in such a way that the reader can identify similar dynamics in his or her own experience. Again, determination of the quality of the theory must rest, at least partially, in the eyes of the reader. 


\subsection{Conclusion}

During this chapter, the interpretive, qualitative, grounded theory methodology utilised for the empirical section of this thesis has been described and justified, and the epistemological implications discussed. The ontological assumptions underlying this mode of engagement - namely that reality is socially constructed - were made explicit, and in the light of this, the application of an organization culture perspective on morality in green marketing was set out. It was thus described how the empirical study would be approached as a cultural analysis, thereby focusing attention on cultural artifacts and dynamics, and on how cultural meanings were influenced by political and symbolic elements.

This form of research method is unusual in the study of marketing and morality, and one of the principal contributions of this thesis is that it takes a new and original approach to empirical research in this area. However, the benefits of the approach utilised here are not simply that it is under-developed in this area, but that it can be seen to be extremely appropriate for many of the questions that are of relevance to this area of study, and more specifically, for the investigation of the broad research questions presented in this thesis. Moreover, the depth of analysis and cognizance provided by this mode of engagement makes possible the contribution of critical, scholarly insights to the sub-fields of business ethics and green business which in the past have been notable for the amount of criticism levelled at their lack of critical depth and methodological rigour.

The results and theoretical propositions which have emerged from the analysis of the case study data are set out in the chapters which follow. The intention in 
chapters 5-7 has been to present theory grounded in data for each of the three organizational forms for which case studies have been constructed; hence they relate to conventional business organizations, social mission companies, and business-NGO collaborations, respectively. The results presented in these chapters, although not the only 'story' that could be applied to the data collected, are regarded by the investigator as the most plausible and persuasive interpretations of the data, and the best maps through the observed fields. The constructs and storylines have emerged following an exhaustive and highly iterative process of constant comparison between theory and data. In chapters 8-9 more general theoretical propositions are set out and discussed - these relate to the case study data taken as an aggregate rather than individually. Again, a method of constant comparison has been used to ensure that these results are as valid as possible. 


\section{Conventional Business Organizations}

\subsection{Introduction}

In this, the first of the chapters reporting results of the empirical research carried out as part of this study, findings relating to two case studies are presented: Boots the Chemist (BTC) and ITT Flygt (Flygt). Taken together, they provide cases in both consumer marketing (BTC) and industrial marketing (Flygt), and hence represent a set of comparative cases 'nested' within the overall comparative case framework. They are regarded here as 'conventional' business organizations in that they are companies dedicated to maximising growth and shareholder returns, and are neither committed to an explicit social mission, nor directly positioned as 'green' companies, as are those in the social mission company category.

Despite being depicted as conventional companies, BTC and Flygt should not necessarily be regarded as typical companies, and there is no intention to claim that the results presented here are representative of a wider sample of conventional business organizations. As an exploratory study, the main intention here then has been to elicit important themes and issues which might be subsequently utilised as building blocks for developing theory. Nonetheless there is no reason to suggest that the picture presented here is unique or unusual, and findings have, where appropriate, been abstracted sufficiently for potentially more generalizeable results to be suggested.

Having analysed the data relating to BTC and Flygt, it was found that there were few overlaps and commonalties between the two companies in terms of their 
approach to green marketing, and in the establishment of an attendant moral agenda. In particular, it was found that BTC had a much more explicit, and considerably more developed, environmental programme than did Flygt, at least at the time when fieldwork was conducted. It is also important to note that it was possible to secure far greater access for data collection at BTC than it was at Flygt, generating a great deal more interesting and useful data; consequently much of the discussion here, particularly in the second half of the chapter, focuses on the former rather than the latter. This is not to say that the insights gleaned from the research at Flygt were any less valid, but rather that the research at BTC provided a more substantial contribution to theory development.

The structure of the chapter reflects this imbalance. First, the two case study organizations are introduced, and then in section 5.3 their approaches to green marketing are outlined. Particular reference is made to the role of social responsibility in these approaches. In section 5.4, the cultural dynamics of green marketing in the case study companies are set out, and here attention is focused in the main on BTC. The principal intention of this section is to draw out some emergent themes which help to explain how green marketing practices are enacted within the organization, and what implications this might have for conceptions of morality in relation to marketing. Finally, the main findings of the chapter are summarised and issues of concern for the following chapter are set out.

\subsection{Background to the Case Studies}

Before setting out the analysis of the two cases, some brief biographical details 
of the companies is required in order to describe the organizational context in which green marketing activities have been introduced. We begin with BTC.

\subsubsection{Boots the Chemist (BTC)}

Boots the Chemist is a large retail company founded in 1877 and based in the city of Nottingham. It is a wholly owned subsidiary of The Boots Company PLC which operates a group of nine companies in total ${ }^{24}$. In 1996 BTC had a turnover of $£ 3 \mathrm{bn}$, around 50,000 employees, and operated over 1100 stores. As well as being the largest UK chemist, BTC was a market leader in many areas of its business including healthcare, cosmetics, toiletries, baby consumables and film processing.

Despite continued success and sustained growth during the twentieth century, the decade previous to when fieldwork was conducted had seen the company go through a process of modernisation, re-structuring and re-organization. This could be seen to reflect a drive towards becoming more competitive after a period of unsatisfactory performance in the early 1980s (Churchill, 1984), and also a re-orientation towards the new Boots Company core objective of increasing shareholder value (Vander Weyer, 1995). Crucially, BTC had begun to consider its key competitors to be the large retail multiples, such as Sainsbury's, Tesco, and Marks \& Spencer rather than other smaller retail chemists, such as Superdrug and Lloyds (Vander Weyer, 1995).

\footnotetext{
${ }^{24}$ At the time when fieldwork was conducted other members of the Boots Group were AG Stanley, Boots Contract Manufacturing, Boots Opticians, Boots Healthcare International, Boots Properties, Children's World, Do It All, and Halfords. Accounting for around two thirds of the total group activity and profits, BTC was, and is, the dominant member of the Group.
} 
Notwithstanding its commercial fortunes, it should be noted that BTC has been historically linked to corporate philanthropism, particularly through its founder Jesse Boot. According to Boot's biographer, Chapman (1974), there can be little doubt that, along with many other wealthy industrialists of the time such as the Cadbury family, and his friend William Lever, Boot was a highly religious man (a Methodist) with a strong social ideology. He is credited with being a great benefactor of his home city of Nottingham and, along with his wife Florence, did much to improve the welfare of his employees during a time of considerable working class privation. When put in such a context then, BTC might arguably be regarded as having been imbued at an early stage with values of social responsibility, albeit of a highly paternalistic nature. However it is clear from Chapman's (1974) analysis that although these were important concerns for the company's founder they were not substantially instrumental in the founding of the company, and it was only as the company became established that concerns for social welfare began to be addressed. Hence it should not be regarded as a social mission company in the same way as those in chapter 6 . Nonetheless, like several of its contemporaries, BTC does still retain a reputation for good corporate citizenship, although as Rowlinson and Hassard (1993) have shown, this could equally be explained in terms of a pragmatic management invention of a corporate culture as it could an 'enlightened' approach to business.

\subsubsection{ITT Flygt}

ITT Flygt Ltd was established in 1958 to provide for the supply, delivery, installation, maintenance and hire of submersible electric pumps and mixers in the UK. ITT Flygt Ltd is a wholly owned subsidiary of the ITT Flygt Group - a multinational based in Sweden and owned by ITT Industries as part of its ITT 
Fluid Technology Corporation. This case study however is based only on the UK subsidiary, ITT Flygt Ltd, which is a medium sized enterprise based in Nottingham with a turnover of approximately $£ 30 \mathrm{~m}$ (1995) and around 300 employees.

At the time of fieldwork, Flygt was the market leader in submersible pumps and mixers, and the brand had been developed as a high price, high quality product at the top end of the market. In recent years Flygt had seen its market expand beyond its traditional base in the water companies to include end-users in industries such as paper and pulp, construction, leisure, chemicals, tanneries, as well as SMEs across various sectors.

\subsection{Green Marketing}

In terms of their principal products and markets, the two companies were clearly in very different positions in relation to green marketing. BTC as a chemist and retailer of cosmetics, toiletries, gifts, food products etc. certainly could not be regarded as operating in a 'dirty' industry, but many areas within its scope of operations could be environmentally controversial. Moreover, a number of retailers of various kinds had been at the forefront of the publicity (both positive and negative) surrounding the green business movement in the UK (Simms, 1992; Murphy, 1996b). Flygt, on the other hand, as a supplier of submersible pumps widely used in the 'environmental' industry, could be regarded as a provider of “absolute green products” (Peattie, 1995:181). 


\subsubsection{Boots the Chemist}

Despite its size and importance in the UK retail industry, BTC has been subjected to little research or scrutiny regarding the greening of its operations in the 1990s, particularly in comparison to its competitors in the grocery sector (see for example, Simms, 1992; Taylor and Welford, 1994; Fineman, 1996). However, the company could be seen to have a fairly well advanced environmental agenda, albeit within a relatively 'clean' area of business. Although several respondents indicated that BTC had introduced a few environmentally friendly practices a considerable time ago (such as the re-use of transport packing materials) it was clear that this had been done so on grounds of cost effectiveness rather than environmental concern. An avowedly "coherent" and "structured" approach to the environment then had not been attempted until 1990 when a dedicated Environmental Co-ordinator position was established within Merchandise Technical Services (MTS), the section in the company responsible for product quality. The first environmental policy statement was subsequently issued in 1991, and internal environmental reporting was begun in 1993, with the intention, according to the environmental co-ordinator, of setting up an external reporting procedure across the Group, "hopefully in a couple of years". At the time of fieldwork in 1995/6 there were three dedicated environment positions in the company, as well as a number of "green teams" comprising of volunteers throughout the company. The role of these green teams has been defined by the firm as: "to investigate opportunities for further environmental improvement and to ensure the implementation of our existing policies at a 'grass roots' level.",25

\footnotetext{
${ }^{25}$ Source: "Environment Information", issued by BTC, 1995.
} 
Organizational greening in BTC can be seen to have been broadly consistent with developments in other large established retail firms (cf. Simms, 1992; Taylor and Welford, 1994; Fineman, 1996). Green marketing activities in the company have spanned the four P's framework, extending into Peattie's (1992) expanded green mix of processes, policies, people and providing information. Indeed, greening at BTC can be said to have impacted to some extent throughout the company, and certainly beyond marketing activities alone. However, it is those aspects of greening which have principally centred on marketing concerns - most notably new product development, corporate communications, promotions, labelling and pricing - which are of particular concern here ${ }^{26}$.

Managers attested to the fact that environmental product concerns in the company related only to own-label goods, whereas proprietary brands could not be similarly influenced. Among these own-label products, the principal environmental developments had been the incorporation of recycled materials into product formulations (e.g. for video tapes, dustbin liners and kitchen knives), the high profile and very successful introductions of natural cosmetics ranges (Natural Collection, launched in 1988; Global Collection, launched 1993), organic or sustainably harvested product ranges (e.g. baby foods), energysaving electrical products (e.g. short-boil kettles), and various products using recyclable and re-usable components. Along with changes in product formulations, BTC had also attempted to "audit" and subsequently reduce

\footnotetext{
${ }^{26}$ Interestingly, respondents themselves did not refer to their company as employing "green marketing" which appeared to carry a pejorative meaning in BTC akin to Peattie and Ratnayaka's (1992) "green selling", i.e. using the environment as a selling point without any appreciable alteration to the product.
} 
packaging, as well as introducing communications initiatives such as an inhouse eco-label (the "world symbol"), collaboration on the FSC timber eco-label through the WWF 1995 Plus Group (see chapter 7), provision of on-pack environmental information, and the publishing of environment brochures and leaflets.

Environmental concerns however clearly made little impact on pricing decisions, and executives regularly claimed that green developments had to be shown to be cost effective before they could be introduced:

Everything doesn't have to make a profit. But at the end of the day, most - well I'd say all - of the successful environmental issues [in BTC] are cost effective. We're doing it because it's sound business sense.

The avowed intention of the company then was to maintain prices whilst attempting to introduce incremental environmental improvements in product formulations. Hence, both premium and penetration pricing were assiduously avoided, reinforcing executives' claims that at BTC the intention was not to highlight the environment as a prominent marketing issue, but to render it as “just another part of doing business".

This attitude was also reflected in the company's overall green marketing strategy. Despite a range of greening activities, few, if any, of BTC's products could be said to be marketed on an explicit green platform, or positioned to appeal directly to 'green' or 'ethical' consumers. This might be regarded as muted greening, a strategy for green marketing whereby environmental 
considerations were incorporated into business practice, but where external environmental promotions were low key and predominantly public relations based. Hence in BTC, environmental "auditing" or assessment procedures had been introduced (e.g. store environmental reviews, supplier questionnaires, and a packaging audit) whereas little emphasis was afforded to corresponding green claims on product packs or in advertising. However, environmental responsibility was emphasised relatively strongly in press releases ${ }^{27}$, information packs, and through local community and philanthropic gestures ${ }^{28}$; equally, internal communications were seen to be fairly well developed with an annual environmental report, a regular environmental section in the in-house magazine, environmental newsletters, and informal communication through the green team network.

It could be argued that BTC's 'Natural Collection' and 'Global Collection' cosmetics ranges constituted important exceptions to this muted strategy. Certainly commentators have tended to stress that these are "environmentally friendly" products intended both to imitate and to compete head on with the Body Shop's avowedly green ranges (see Marketing Week, 1991; Davidson, 1993; Mintel, 1995) - an argument at least partially acknowledged by the firm's managing director (see Vander Weyer, 1995). However, respondents from the company emphasised that none of their products were marketed as green - and that this included these two cosmetics ranges, e.g. "(Natural Collection) wasn't

\footnotetext{
${ }^{27}$ For example, "Boots Takes a Lead on 'Green' Video Tapes", 2/10/95; "Boots Reduces the Christmas Wrapping", 6/12/95; "On the Twelfth Day of Christmas...Boots Help Convert Used Christmas Cards into Trees", 8/1/96. Dates indicate day of release by BTC.

${ }^{28}$ These are summarized in the Boots Company PLC's annual reports and in "Boots in the Community" also published annually by the company.
} 
an environmental range, it just had natural ingredients." Indeed, direct green claims were relegated to second-order appeals for these products, both in advertising and in packaging texts, as might be expected within a muted green strategy. However, the obvious similarity with the Body Shop branding suggests a deliberate attempt to create substantial indirect signification of environmental credentials, such that these products might appeal to ethical or environmental purchasing behaviour of some kind. Hence executives were able to articulate a consistent and coherent muted green strategy (what was actually claimed), despite reasonable success on a green platform in this product area (due to what was implied through copycat branding).

According to respondents this muted strategy was principally driven by changes in the marketing environment. It was generally accepted that it had become increasingly important for BTC to remain credible and responsible in the eyes of the public compared to their competitors but at the same time, as one respondent put it, to avoid risking "putting our heads above the parapet" which may have incited unwanted or inappropriate attention from outside bodies. This had meant that the firm could not be seen to be doing too little, as this may have led to reputation problems; equally, it was seen by executives as potentially dangerous to, as they put it, "go around shouting about it like other companies have" since this could have drawn attention from latent critics. Such a balancing act was regarded as a pragmatic response by the company to the perceived (and often conflicting) pressures of increased external scrutiny, market pressure, consumer confusion/scepticism towards green claims, and increasingly onerous regulation. Most interestingly, the consumer was depicted both as "the key driver" of organizational greening, and also as a significant barrier to more explicit green 
marketing due to their antipathy towards green products ${ }^{29}$. Green branding was seen as sure to "cause sales to bomb", implying that consumers were indeed negatively motivated towards purchasing green products. In the main this was seen as a result of a) consumer doubts over the quality of green products and b) a lack of trust in green claims. Specific citations of these problems tended to rely on examples from the cleaning products and detergents sector, and in particular on green washing powders and liquids which were depicted as seriously underperforming ${ }^{30}$. Thus, rather than attempting to use the environment to present an overtly positive corporate image, "we had to manage the environment such that it didn't damage our reputation" - a more cautious and conservative reputation management approach ${ }^{31}$. These findings provide strong supporting evidence for Wong et al.'s (1996) conclusions that UK consumer marketing firms were playing down their environmental credentials in response to an emergent consumer backlash in the early-mid 1990s. According to Wong et al. (1996), this backlash had largely arisen from a mismatch between consumers'

${ }^{29}$ It should be noted that these assertions from executives tended to be based either on secondary research (such as Mintel reports) or anecdotal evidence. The company itself had conducted no market research at all on environmental issues.

${ }^{30}$ The main example cited of this phenomenon actually relating to BTC products however was the case of a range of dustbin liners which, having failed under green branding, were successfully re-launched as a conventional product despite an identical product formulation.

${ }^{31}$ Such concern with its environmental reputation was almost certainly intensified by BTC's recurrent problems with animal rights activists, hence: "where we've been hammered in the past is animal testing." This involved store picketing in the 1980s (The Times, 6/11/82, p.3), fire bomb attacks on its stores in 1993/4 (The Times, 29/12/93, p.2; 30/12/93, p.1/6; 8/4/94, p.8; 25/8/94, p.5; 17/9/94, p.2), and disruption of the Boots Company AGM's throughout the early 1990s (The Times, 24/7/92, p.2; Financial Times, 23/7/93, p.22; The Times, 22/7/94, p.25). However, since the Boots Company's sale of the Boots Pharmaceuticals division in 1995, this had not been repeated. 
expectations and the reality of firms' product offerings, much of it due to ineffective communications and promotional policies.

In a sense then, the strategy for greening adopted by BTC cuts across Peattie and Ratnayaka's (1992) off-the-peg potential strategies: changes were issue-based and cautiously attuned to the exigencies of specific markets and market environments rather than being simply "piecemeal". Hence, on issues such as responsibility for packaging the company might be regarded as having adopted a "defensive" strategy; on animal testing a "knee-jerk reaction" strategy; on natural ingredients a "follow the herd" strategy; on wood sourcing a broader "greening the company" strategy; and so on.

\subsubsection{ITT Flygt}

Flygt's approach to incorporating environmental considerations into its marketing activities revealed a yet more curious picture. The Sweden-based parent company, ITT Flygt, could be seen to have publicly presented itself as an intrinsically green company, due in the main to its marketing of environmental technologies for water treatment. For example, of the ten major stories featured in the 1995 Annual Report, no less than seven were concerned with the environment. In addition, the marketing report in the same publication claimed:

We are also a leader in environmentally-related technology. Environmental considerations play an important part in both product development, and generally at work, where the company has a comprehensive Environment, Safety and Health program in place. 
This emphasis on the environment was repeated in company magazines and other publications for customers and various stakeholders that were published by the parent company.

In stark contrast to this polished green image of the parent company, respondents at the UK subsidiary expressed little conviction that their business had developed any kind of coherent environmental programme outside of a "general awareness" of the issues. Hence: "To be honest with you, I'm not sure we do have an environmental agenda," ... "We don't get involved in the environment all that much,"... "The environment is only marginal to our business." Indeed, whilst the use of the company's products was clearly environmental in nature (e.g. in the treatment of effluent) this was afforded scant significance by executives in Flygt. Respondents suggested that the equivalence of environmental credentials for competing firms in this respect made such concerns fairly redundant. In addition, customers were presented as having a very limited interest in environmental issues, and as a result little adaptation had been necessary in marketing terms. There was though some suggestion that environmental considerations were beginning to feature in customers' demands, although respondents were quick to emphasise that this was certainly due to increasing regulatory pressure rather than any kind of moral impetus. Indeed, it was inconceivable to respondents that the environment would feature in industrial marketing exchanges unless driven to by external pressure: "the customers will often be forced into doing it. No one does it unless he has to."

This is not to say that respondents made no claims at all for the inclusion of environmental concerns into marketing activity: the environmental performance 
of product features such as noise, oil and coatings were all clearly of consequence to the overall product offering. Some opportunistic public relations activity had also been initiated and this had seen the company involved in public programmes with varying environmental consequences. Moreover, some communications and promotional texts made at least limited reference to the environmental qualities of the firm's products. This was principally by specifying the components' accordance with regulations of various kinds, and by some reporting of the practical role of their products in environmental management systems or in meeting customers' environmental objectives. The 'caring' or custodial type themes typified by BTC were not however in evidence, suggesting that consumer and industrial marketing firms might adopt different approaches to the incorporation of environmental themes in communications texts.

Overall, the green marketing strategy adopted by Flygt was probably closest to what Peattie and Ratnayaka (1992) describe as a "knee-jerk" strategy: compliance with regulation and response to strong external pressures. Hence, green marketing at the company was fragmented, unmanaged and increasingly opportunistic in the context of potential changes in the marketing environment. Respondents made no attempt to hide such greening initiatives behind protestations of ethical motives:

The [customers] are trying to change their image so that they are seen as green ... and we can be part of the bandwagon to sell some product and develop new markets. 


\subsubsection{Green Marketing and Corporate Social Responsibility}

In many ways then, it is difficult to ascribe an overall social responsibility strategy to the practice of green marketing at these two companies. However, at BTC where the environmental agenda was considerably more developed, a clear discourse had begun to be developed around the greening programme. Hence, the recurrent theme pervading the organization, evident both in formal documentation (reports etc.), informal documentation (newsletters, training videos etc.) and routinely incanted by executives in the company was of the form:

Sound environmental sense makes good business sense.

This theme could be seen to be firmly located in the philosophy of enlightened self-interest, i.e. where 'ethical' actions are taken in order to yield long term performance benefits. Correspondingly, it had tended to be articulated particularly with reference to cost savings that could, or had, been made through, for example, packaging reductions and energy efficiencies. There were also significant incidences when respondents would rationalize all organizational greening practices in terms of economic performance criteria or "good business sense".

Whilst this notion of enlightened self-interest was clearly central to green marketing in BTC, it had also begun, to some extent, to emerge in relation to Flygt's incipient greening policy. Although at the time of fieldwork, this could not be said to constitute a clear moral discourse at the firm, there were certainly signals from the parent company that such an instrumental, self-interest 
conception of the environment was likely to be promoted. Hence the environment was presented in much the same way as at BTC:

Good environmental management can pinpoint opportunities for cost savings. It encourages energy efficiencies and reduces waste. It gives you a nice, clean corporate image. In short, it's good for your bottom line ... and rather than being a constraint, it just makes good business sense. ${ }^{32}$

Clearly then, green marketing at the conventional organizations rested on notions of self-interest. How these then came to be established, legitimated and subsequently integrated into the fabric of the company however is not evident at the current level of analysis; nor indeed does it provide clear insight into what implications this might have for the marketing strategies and tactics adopted by the companies as revealed above. The following section then seeks to explain in more detail the processes underlying the introduction of green marketing programmes at BTC and Flygt. In so doing, the aim is to explore how attendant notions of morality were given or denied expression within this process, how the self-interest discourse came to be accepted, and how this can be related to manifest marketing phenomena.

\subsection{Cultural Dynamics of Green Marketing}

Overall, the prevailing ethos of the conventional organizations was characterised by respondents as conservative and business-like. They were seen as typified by well-established and fairly rigid attitudinal and behavioural norms which

\footnotetext{
${ }^{32}$ Source: ITT Flygt, 1995 Annual Report, pp. 12-13.
} 
reflected the values of sizeable populations of long-term employees ${ }^{33}$. In the context of such orthodoxy, the environment might be considered to be a potentially radical introduction into the cultural knowledge of the firm and its members (Fineman, 1996). Indeed, respondents from both companies tended to present the extant corporate culture as potentially resistant to greening, unless it could be shown to be consistent with existing commercial priorities and norms. However, it is important to note that at the time of introduction for the companies' nascent environmental agendas (for BTC in the early 1990s; for Flygt in the mid 1990s) green and/or ethical marketing issues were still considered 'new' to the organization. Hence moral meanings for the environment were open to interpretation and were, to a larger extent, negotiable.

At a micro level, it was evident that the process of contesting and institutionalising such green meanings centred around the presence or otherwise of certain key individuals which, after Drumwright (1994), shall be referred to as environmental "policy entrepreneurs". These were found to be present in BTC but not in Flygt. Therefore, significantly different dynamics were revealed in the two companies, most notably in the sense that only in BTC could this process be regarded as managed in any way. The following section looks at the role of policy entrepreneurs in the development of the corporate green agenda in conventional companies before going on to analyse the processes associated with these actors in BTC in relation to the construction of 'the environment' and institutionalising attendant moral meanings.

\footnotetext{
${ }^{33}$ Vander Weyer (1995) for example depicts BTC's management as "notably homogenous in its personal style and background."
} 


\subsubsection{Policy Entrepreneurs}

Whilst the existence and importance of environmental managers, advocates, leaders, and/or champions has been fairly widely recognised in the organizational greening literature (see for example, Elkington and Burke, 1989; Dillon and Fischer, 1992), little examination has emerged concerning their personal and professional roles in the process (Fineman, 1997). However, Drumwright (1994:4), in her study of socially responsible organizational buying, provides an important and illuminating description of such "policy entrepreneurs". In many ways these are similar to the key environmental protagonists discovered at BTC:

Policy entrepreneurs... usually were facile at both motivating people and making the system work for them.... had tenacious persistence and a high energy level.... were undaunted by resisters and operational problems.

At BTC, policy entrepreneurs or environmental champions can be seen to have fulfilled similar roles in fuelling and driving the internal green agenda. Indeed, without strenuous campaigning on their part, far less positive environmental marketing activity would have been contemplated by the company. Such campaigning was mainly achieved through energetic attempts to maximise personal interaction between themselves and their less environmentallymotivated colleagues, and through constantly re-iterating and re-vitalising environmental themes:

I get round all of what we call our group product managers .... as frequently as possible. And what we have to do is ... get those meetings in the diary and get 
out to the business centres. And that's the only way to do it. You get yourself about. Its hard work but its got to be done.

Executives also attested to the importance of environmental protagonists being highly motivated and, in particular, being persistent in seeing environmental initiatives through. Hence, "everything's possible - it just depends if someone is willing to put the effort in." In addition, respondents also highlighted the importance of their exercising subtlety and guile in the "highly political" process of securing support and acceptance for environmental initiatives at BTC.

Interestingly, environmental protagonists at BTC did not particularly exhibit an evangelistic sense of mission towards the greening of their organization. Executives with direct green marketing responsibility appeared to harbour a range of environmental standards and ideals. Whilst some exhibited a clear concern for the environment, others were obviously less personally committed. Environmental responsibilities were generally taken on more for career advancement than they were for moral or environmental commitment, evidenced by statements such as, "I certainly never had any personal desire to get involved in the environment," and "I wanted to get the environment on my $C V$." The personal moralities of BTC environmental executives then were on the whole less instrumental in the adoption of their policy entrepreneurship role than were traditional drives for organization success: the role was simply regarded as a necessary one to do a good job in a new and problematic organizational function. This corresponds with existing UK evidence (Fineman, 1996; 1997), 
but stands in contrast to that from the US (Drumwright, 1994) ${ }^{34}$, suggesting the possibility of a country effect: US managers might be more likely to present themselves as emotionally committed to greening than their British counterparts.

In contrast to this situation in $\mathrm{BTC}$, no such environmental policy entrepreneur role appeared to be filled at Flygt. In general, direction and meaning seemed to be derived from intermittent and irregular signals from the parent company rather than from an internal source. This however appeared to have had little impact on the cultural knowledge of the firm or its employees, reflected in turn by the general lack of environmental awareness in the firm. As one manager put it:

I don't think that making environmental products will make you more environmentally conscious. You may become more aware of some of the problems, but you do tend to get blasé about it.

Conversely, the issue of health and safety $(H \& S)$ appeared to be prominent in respondents' consciousness. Not only was it widely attested to be central to the firm's operations, but respondents suggested that it was one of the key issues which set Flygt apart from its competitors. Hence, "our health and safety is second to none"..."the company has used safety as a selling point"... "because of the health and safety rules on site, we make quite a show of it".

Equally, in much the same way that the progression of the environmental agenda

\footnotetext{
${ }^{34}$ Drumwright (1994) in fact regards an ethical motivation as intrinsic to the definition of a policy entrepreneur. This stricter definition is not utilised here.
} 
at BTC apparently relied on environmental policy entrepreneurs, respondents from Flygt suggested that the H\&S issue had been propelled to such prominence by the commitment of particular individuals:

The Health and Safety Manager is very hot on safety ... she's been here about five years I think, and she's made a big difference.

Therefore, the presence of H\&S advocates and absence of environmental advocates had clearly had a significant effect on the cultural knowledge of the firm and its members. Without such championing, the environment had received little attention in Flygt compared to H\&S. As a result, work-related environmental meaning on the part of respondents largely mirrored the firm's mode of environmental management, i.e. unfocused, scattered and idiosyncratic. In search of some kind of frame of reference for environmental activities in the company, respondents tended if anything to link the environment to this highly prominent H\&S function. However, as one respondent from H\&S lamented, this assumption was decidedly misplaced:

Environment is becoming more high profile. The parent company has just done their ISO 14000 ... and so everyone is phoning us up and asking us about it ... but we don't know any more than they do!

\subsubsection{Processes of Institutionalisation}

Policy entrepreneurs then were clearly integral to the development or otherwise of an explicit environment agenda in the conventional business organizations of this study. Evidence suggests however that such internal marketing of green 
initiatives is likely to be a difficult task, particularly in the context of confusing or unsupportive market signals (Wong et al., 1996; Fineman, 1997) and/or a lack of attendant cultural endorsement (Drumwright, 1994; Fineman, 1996). This next section then seeks to reveal in more detail the processes by which such individuals attempted to incorporate the environment into their organization, and in particular how this may have affected the notions of morality attached to the company's green marketing programmes. Accordingly, attention is focused on BTC rather than Flygt since it was only in the former that such individuals were discovered. Analysis of data relating to BTC revealed in particular that policy entrepreneurs appeared to have developed a set of articulated and unarticulated micro-political manoeuvres which were generally believed to constitute a recipe for success for the selling of green initiatives within the organization. Broadly speaking, these can be categorised as image making, moral framing, narrative surfing, and value dissemination.

\subsubsection{Image Making}

It was recognised by policy entrepreneurs in BTC that in what they regarded as a relatively conventional corporate milieu, and one not particularly open to radical ideas, the environment as a concept could be seen as in some way inappropriate or challenging to the status quo if associated with the wrong images. Accordingly, the position and status of environmental executives compared to more traditional roles in the firm was an issue of some consternation to the post holders. In particular, they related how in the early stages of BTC's environmental policy implementation, they were regarded by most of their colleagues as "out on a limb", and considered peripheral to the main business of manufacturing and marketing products. One respondent for example described 
their colleagues reaction thus:

"I think we were tolerated... just leave them alone ... they are doing a good job elsewhere ... just let them get on with it."

In response to the possibility of being marginalised and subsequently manoeuvred away from having any real organizational respect and power, environmental executives consciously, and at times unconsciously, appeared to practice a subtle form of personal and group image making. Most obviously, they sought to distance themselves from what they regarded as the corporately unacceptable image of the environmentalist - "the beads and braids brigade"... "the beard and sandals lot" ... "the cranks". A deliberate adoption of a sober and "professional", "businesslike" appearance and manner was supplemented by an avoidance of "emotive" or ethical arguments in favour of "rational", technical ones. This can be summarised as the "responsible management" image:

[The first environmental executive] was meticulously carefully to make sure that he was always taking a very responsible management approach, and so he built up a reputation that this isn't just some sort of open-toed sandal, you know, thongs and braids brigade going in... Its that perception thing again. I think its grown up that we do take a professional approach and I make sure that, you know, that sticks.

The need to be seen as professional managers in the same way as their colleagues was felt acutely by environmental executives in this study. It was important to them that they fitted into the corporate culture which they routinely 
depicted as "professional" and "businesslike". This cultural integration could be argued to have served two purposes. First, it provided managers with the correct and unchallenging image with which to pursue initiatives which may otherwise have been regarded as suspicious, inappropriate, or controversial. As a result, they were able to progress relatively unhindered by their non-traditional role towards the customary rewards of organizational success, namely job security and career advancement. Second, it also enabled these individuals to engage their organization more intimately, to express loyalty to their company and pride in their job, and consequently to contribute to the satisfaction of their needs for respect and belongingness (Fineman, 1996).

A possible side-effect of these acts of image-making appeared to be some form of minor "moral stress" (Bird and Waters, 1989), brought on by the relinquishing of personal moralities to the "enacted" morality of the organization (Fineman, 1995; 1997). In some situations, clear differences could at times be discerned between a) the moral certainties articulated in response to questions regarding the company's policies and practices and b) admissions of moral ambiguity and outcome uncertainty when questioned on respondents' personal opinions. In the most compelling example of this private-public tension, one respondent had claimed not to be an environmentalist whilst the discussion was focused on company policies, whereas later in the interview, when the respondent's personal moral position was sought, this claim was retracted, even to the extent of emphasising their membership of environmental organizations and their resignation from a previous company on the grounds of maintaining their environmental conscience. 


\subsubsection{Moral Framing}

This image of the professional manager was bolstered in a number of ways by environmental executives in BTC, but it was most apparent in the arguments which they presented in order to describe and justify the adoption of environmental initiatives. In the main, executives were at ease when discussing environmental marketing projects which could be readily subsumed within a context of cost savings and other commercial justifications. These had generally been the issues which BTC had tackled earliest in its environmental programme, the "low hanging fruits" of win-win solutions which offered bottom line benefits along with reductions in environmental impacts. However, even those activities which may have been broader in scope and which may not have so obviously generated immediate quantifiable benefits for the company (such as environmental "auditing", and efforts to improve environmental labelling) were also assimilated within a highly instrumental conception of the environment. Indeed, managers routinely emphasised the lack of conflict between environment and economics, and they frequently related back to the enlightened self-interest theme of "sound environmental sense makes good business sense."

Interestingly, at times this logic was even inverted such that environmental benefits were argued to only occur in the context of economic benefits, e.g. "the cost is often a good guide to [the best environmental solution]"... "if its not making sense economically, then it is probably not making sense environmentally, and so we haven't found really that that causes us a conflict." This finding is crucial. These managers are claiming that the environmental impacts of competing solutions can be best assessed according to their relative cost. Hence, if a proposal costs money without attracting sufficient returns, then 
it isn't environmentally appropriate in any rational way. This is a curious, perhaps disturbing, rationale that can perhaps only be adequately explained by the total immersion of these managers into the cost and enterprise culture of their departments.

In general then, managers' rationalisations for environmental activities almost exclusively emphasised commercial considerations rather than ideological, ethical, cultural, or personal motivations, even though there was the very real possibility that organizational resources may have secured a better economic return elsewhere. The invocation of consumer need was much in evidence, and the careful interpretation of, as yet inconclusive market research, appeared to be a powerful legitimating device in support of executives' green projects ${ }^{35}$. Such "rational" and "commercial" arguments were seen as central to the responsible management approach and other "emotive" arguments were condemned in comparison:

I don't react emotively; I react based on scientific evidence that is put in front of me. So that if you take that approach, it is often a lot easier to combine the sort of professional, commercial approach to environmental management as opposed to the emotive, reactive sort of approach.

The need for executives to negotiate the embedded culture of the organization in securing support and commitment for environmental marketing initiatives can be seen to have had the effect of them enacting a moral frame that effectively castrated moral meanings from the environment. Clearly, from an ethical point

\footnotetext{
${ }^{35}$ For a more thorough analysis of this phenomenon, see Brown and Ennew (1995).
} 
of view, this would correspond more with teleological conceptions of morality than deontological ones. Moreover, notions of moral product augmentation in this scenario appear to be very much divorced from considerations of ethical decision making within the organization. The commodification of social and ethical issues may indeed fundamentally alter those issues, at least for the individuals and companies involved in the process of developing and marketing such products. Hence, it would appear in BTC that the marketing process itself acted to neutralise concepts of morality or ethics, or rather amoralized green marketing. Whilst the invocation of commercial arguments for specific projects can be seen to be the most obvious manifestation of this process of moral castration, more subtle elements, such as the setting out of moral boundaries and the avoidance of moral vocabularies can also be detected which underpin and interplay with this approach.

\section{Setting Moral Boundaries}

As the main environmental reality definers in BTC, policy entrepreneurs had attempted to set the boundaries within which the environment was considered to be a social or ethical responsibility of the firm. It is revealing then that many of these individuals made a distinction between issues which were "ethical" and those which were "environmental". This was evident despite specific reference in their original environmental strategy document to the intention of "developing environmental policy within an existing reputation for ethical business". This reluctance to categorise environmental products within the rubric of ethical business was manifested in comments such as those relating how the prominence and "fashion" for environmentalism was beginning to be overshadowed by that of ethical issues: "its difficult keeping the spotlight on 
core [environmental] issues. Now we're having to move into the ethical thing." This position was re-iterated even more explicitly when respondents were asked to clarify the scope of the company's environmental policy, and also how this translated into defining their own functional responsibilities:

We... are careful about the language we use - ethical and environmental... but there are some ethical issues which are fairly close... They are actually reporting to different boards of directors, so as a company we do actually separate out the two.

It is clear then that despite the assertions of more senior managers, quite specific moral boundaries had been set out among executives at BTC. As much as possible, "ethical" issues were marginalised, and frequently excluded, from the mindsets of executives who had to deal with "environmental" products and problems on a daily basis. Those environmental products which they had to contend with were therefore not consciously framed in terms of explicit moral responsibilities or dilemmas, but rather as technical problems which demanded technical solutions. Moral reflection, as much as possible, was apparently avoided. These findings are broadly in keeping with those of other qualitative studies in the business ethics field where managers have been found to avoid moral discourse (Bird and Waters, 1989) and become de-sensitised to the moral dimension of their activities through bureaucracy, formalisation and task repetition (Jackall, 1988).

\section{Semantic Suspects}

Content analysis of interviews and company documentation revealed that at its 
most extreme, this avoidance of ethical themes in environmental discourse at BTC could take the form of a wholesale avoidance of certain words and concepts. These can be subsumed within the category of semantic suspects, i.e. those symbols which may have translated meanings which environmental executives felt would damage both their personal image, and the status of environmental business in BTC generally. In particular, organizational conceptions of the environment were channelled away from ethically contestable notions such as 'deep ecology' concepts of sustainability, biodiversity, and ecocentrism. For example, although arguments for sustainability have been held to be less confrontational towards business than many other environmental positions (Peattie, 1995), they were still assiduously avoided by environmental executives at BTC. The term itself was almost never used by respondents in interviews, nor did it appear in external communications. Indeed, in the BTC environmental policy statement, sustainability was not directly mentioned despite the use of a phrase - "our aim is to meet current needs, without compromising the ability of future generations to meet their own needs" - which was almost identical to the much-quoted World Commission on Environment and Development definition of the term (see footnote 16). The avoidance of sustainability here was all the more striking for the inclusion in its place of its principle tenet; it might thus be reasonably argued to be the result of a deliberate and calculated semantic choice.

At one level, this reflects BTC's muted greening strategy, and respondents were quick to emphasise the potential pitfalls of using terms where definitions might be open to differing interpretations. Also, at a deeper level, it reflected attempts by environmental executives in BTC to re-frame the environment in order to 
present it as a normal "rational" business function rather than a soft "emotive" issue:

As soon as you mention [sustainability], people's eyes glaze over. If your are talking to senior managers, they think you are going back to the hippie days, you know: Sustainability? We are running a business to make profits. Its not about furry things.

Whist interview protocol was designed so as to focus discussions directly on environmental issues, it was somewhat surprising that executives made little or no attempt to incorporate the environment within a discussion of social responsibility or corporate morality. Morality terms such as 'ethical', 'moral', 'responsibility', 'duty', 'right' and 'wrong' were rarely if ever used by respondents. The company's twenty-page environment information pack also avoided direct morality terms, preferring instead to emphasise the size and importance of the company and its policy of "caring" for customers. In general then, executives at BTC involved in the processes of environmental marketing acted to neutralize morality, or to amoralize the environment in order to secure organizational endorsement for their activities. This can be seen to have been a deliberate strategy adopted by the first environment manager:

You have to be realistic. Things like sustainability - unless you dress them up in more business language, they are going to be resisted. You have to accept that there are certain words and phrases and approaches that turn the locks in a business. And if you can't get the right managers to buy into what you are doing, because you are using the wrong language or the wrong keys, then it doesn't 
matter how sensible you are, or what you are trying to push, you've had it.

Hence the muted greening strategy revealed earlier can be seen to have been a product of the way in which environmental protagonists within the company had sought to balance internal and external pressures for and against greening. Their tactics clearly focused on finding a vocabulary for greening which was appropriate for the conservative and cost-focused corporate milieu of BTC, but also one which reflected their notions of professional retailing. The discourse subsequently established appeared almost entirely amoralized.

\subsubsection{Narrative Surfing}

Managers sought also to integrate the environment into the fabric of the company by aligning green marketing activities with existing prominent corporate narratives. This process is referred to here as narrative surfing, since the choice of narrative appeared to reflect the particular demands of the situation, and executives seemed adept at invoking different narratives as needs arose. For instance, the introduction of the environment into everyday operations of the company was compared by almost all respondents to the integration of (total) quality concerns during the $1980 \mathrm{~s}$ :

Environment is very often linked to quality... many people find that it mimics quality maybe 10, 15 years lagged behind where quality is now... so its really from a quality background.

This association of the environment with the quality narrative can be argued to represent an attempt to de-mystify the greening process by comparing it to a 
process that was already known and considered to have been a successful and relatively unproblematic transition for the company. Further credibility was garnered by the fact that the source of most early environmental developments in the company, the MTS section, was also regarded as the quality division - and several key individuals had been involved in both schemes.

It is evident however that environmental policy entrepreneurs did not limit themselves only to attaching the environmental marketing activities to the quality narrative, but rather 'surfed' various narratives as it was appropriate given the particular contextual and political exigencies of the situation. For example, the quality narrative appeared to be principally invoked with reference to new product development, production, and process decisions. When contesting promotion decisions however, the preferred narrative was that of "caring".

"Caring" refers to BTC's umbrella corporate marketing campaign at the time that fieldwork was conducted. The phrases "Who cares?...", "Someone cares..." and "Boots cares" formed the context for all BTC marketing communications at this time. As such the campaign had been adeptly invoked by senior environmental executives in order to contrive an effective bargaining position with senior marketing executives:

The Director of Marketing....accepted as a principle that ... if Boots was not seen to be environmentally sound then the whole Caring campaign had a major flaw in it. Because if you can't care about the environment, something as fundamental as that, how can you care about the rest of it? 
However, the integration of the environment within the caring narrative had not been restricted merely to internal political uses, but can be seen to had also manifested itself explicitly in formal policy documents and marketing texts, with comments of the type, "Caring for the environment and the people in it goes hand in hand with BTC's policy of caring for its customers." Crucially, the use of the caring narrative can be seen to have been used in preference to a competing narrative of social responsibility which may arguably be regarded as having been equally, if not more, applicable in these contexts. Again however, the use of an explicit moral term such has social responsibility may have been adjudged to be overly challenging to the existing corporate ethos whereas caring was both less suspect and more in line with other marketing texts. The use of this narrative thus normalised the greening process.

One final narrative with which respondents can be seen to have fused environmental considerations was that of BTC's cultural traditions. BTC's philanthropic founder acted as a useful narrative for contemporary employees in terms of establishing a meaningful frame of reference for interpretations of, and justifications for, recent environmental activities in the company:

When we issued the environmental policy and we started looking at it in a structured sort of way.... we even related it back to Jesse Boot's days, when he founded the company, and was founding it on a social and ethically responsible premise.

This manifested itself to a greater degree however in various marketing communication texts. Key environmental documents, directed at internal as well 
as external audiences, habitually began by integrating environmental activities within a wider historical narrative (and also invoking the caring theme), using statements of the kind, "Since the Company was founded BTC's philosophy has been to care for its customers, staff and the local community."

In marketing communications then, the philanthropy of the founder was mobilised in order to give credence to claims for a long unbroken tradition of "caring" at the company. In contrast, the living history of the firm, the stories recounted by present employees, provided little insight into this legacy of social and environmental responsibility. Rowlinson and Hassard (1993) refer to this as the "invention" of corporate culture: the process of giving meaning to events through the implication of a largely factitious continuity with the past. Certainly respondents' use of the historical narrative in this study was indeed limited to specific references to the company's founder, and to claims of a well established reputation for concern amongst consumers which had to be fulfilled. In general then, the cultural tradition narrative served as a useful symbolic device for green marketing communications, particularly in terms of creating an historical embeddedness for environmental activities. Moreover, internally it had more currency when translated into a consumer demand, i.e. a reputation for social and environmental responsibility that had to be maintained. Once more this had the effect of suppressing the distinctly moral component (an internal, cultural drive to "care") in order to stress the instrumental (an external demand to "live up to" the existing reputation) - again re-framing the environment as an amoral and "normal" business activity.

In short then, the surfing of appropriate existing company narratives allowed the 
environment to be presented as something which was neither strange (through the quality narrative), nor different (through the caring narrative), nor new (through the tradition narrative). When considered in association with the other emergent themes of image making and moral framing, the practice by which policy entrepreneurs in BTC had sought to secure commitment and support for green marketing is fairly evident. Most notably, it had involved the neutralization of morality in relation to the environment: the intention in the company had been to render the environment as just another business problem with no more and no less relevance to notions of ethics or morality than for any other issue. In the final section focusing on BTC, the analysis moves onto an exploration of the processes by which these established and legitimated environmental values had been disseminated through to areas of the organization outside of the direct contact of the main environmental protagonists.

\subsubsection{Value Dissemination}

Whilst the environment as an issue was clearly not central enough to be at all consequential in recruitment and selection decisions in BTC (other than for dedicated environmental positions), once employees had joined the company several mechanisms were in place by which they could garner environmental knowledge, awareness and meaning. Environmental newsletters, bulletins, and leaflets of various kinds acted as useful vehicles for communication of issues and for raising the profile of the environment within the company; and whilst these may also have included fairly general environmental information and tips, little opportunity was lost to reinforce the prevailing theme of "sound environmental sense makes good business sense". Hence, this message was reiterated in various texts such as the environmental policy statement, a staff 
information video, customer environment leaflets, and so on.

The informal green team network was generally seen as the most important vehicle for raising environmental awareness in the company, particularly in the formative period of greening. As groups of interested volunteers, working outside of company time, these tended to comprise of individuals with some level of explicit moral motivation, e.g. "Its mainly people who are interested in green issues personally" ... "On a personal view, it is something that I am interested in, something that I want to be involved in. I feel quite strongly about it." Green teams consequently concentrated on issues of immediate concern to their members, particularly the establishment of local recycling and re-use systems within individual departments. And whilst they had limited, if any, impact on company policies, even at the most basic tactical level, these teams could be seen to occupy an important role in informing and motivating people about environmental issues generally and also in relation to their particular sections and roles. Policy entrepreneurs then had utilised the enthusiasm of their colleagues to generate a more conducive context for greening activities. Hence they saw the environment as an "empowering tool" with which they had "persuaded a lot of people to do things different ways." Nonetheless, since green team members tended to be involved for personal rather than for professional reasons, they could be as one senior executive put it, "a double-edged sword" that may have impeded the crucial support and attention of line managers. So although the team meetings clearly allowed members to speculate fairly openly about environmental issues and problems at the company, there was also a general recognition that proposals had to be local, practical, quantifiable, and cost effective. This had called for careful management in order to focus the 
teams' attention on parochial concerns, and to ensure credibility of the overall greening project.

Some evidence of the environmental agenda having successfully permeated at store level could be detected, although in a substantially diluted form. The muted greening strategy and the attachment of the environment to existing narratives and discourses within the company meant that at the front line, what little environmental meaning that had been present tended to be lost. Hence, store staff saw issues in terms of costs and customers rather than nature or morality. In this sense, energy efficiencies were seen to be primarily cost-motivated, and products were regarded as being stocked to offer choice rather than because they were of environmental benefit. Despite at least some environmental training having then been activated at store level, knowledge of the BTC's environmental policies and programmes appeared limited, and store staff's comments were typically of the type: "I think they are recycled" ..."I assume its used in some environmentally friendly way"..."I think there was a document or something that we were given in training". This lack of certainty and involvement seemed to be exacerbated by a perceived lack of concern amongst the majority of consumers, and therefore greater personal involvement in the environment was considered to be valid only "where it meant you could give a better service or do a better job."

However, for policy entrepreneurs, the dissemination of values throughout the company was generally not seen as important as was the diffusion of environmental responsibility. Specifically, the intention had been "to get the environment integrated into everybody's day to day's business". This had taken the form of working towards integrating environmental responsibilities into staff 
job descriptions and responsibilities, whilst at the same time preventing the growth of a dedicated environment section. Respondents presented this as akin to integrating total environmental quality through broad cultural change. Equally, in this way it was possible to prevent environmental managers from becoming an environmental "dustbin", i.e. a marginal repository for all of BTC's environmental problems. In this way, the environment could be further decoupled from any notions of a personal moral crusade, and established as "just another part of doing business".

\subsection{Conclusion}

The analysis presented above provides some important insights into green marketing in conventional business organizations, and in particular into the tactics adopted by individuals within one such organization to secure support and commitment for these activities.

First, the extent of the greening programmes in these companies appears to be directly related to their susceptibility to such external threats as regulatory attention, media exposure and adverse publicity. The high visibility of a retailing organization such as BTC was clearly felt to project it into a more vulnerable position than that of an industrial marketing company such as Flygt, despite the latter's involvement in environmental technologies. Indeed, respondents at Flygt presented a picture of only limited environmental awareness and concern compared to that at BTC. Hence, potential threats to corporate legitimacy appear to have more bearing on the attention afforded to environmental issues than do the intrinsic environmental qualities of a firm's products. 
Second, in the absence of any clear evidence of the environment having impacted significantly upon consumers' purchase behaviour, except where required by law, the firms had attempted only limited incorporation of the environment into pricing, communications and promotion policies, although the environmental performance of products was of issue in specific markets. Hence, their adopted marketing strategies might be referred to as 'muted' greening, i.e. claims for green product benefits were low key and predominantly public relations based in order to deflect potential criticisms rather than to attract customers. In BTC at least, protestations that environmental initiatives were consumer-driven appeared to owe more to managers having been socialized into the rhetoric of marketing orientation than it did to manifest policy.

Third, it was found that green marketing in these companies tended to be introduced under a banner of enlightened self-interest. This was typified by the recurrent theme of "sound environmental sense makes good business sense" in BTC. A remarkably similar situation appeared to be arising also at Flygt's parent company, suggesting that the firm's as yet under-developed environmental agenda might emerge within a broadly similar (a)moral discourse.

Fourth, this instrumental conception of the environment was further underlined by the efforts of policy entrepreneurs to establish support and commitment for greening, at least in BTC. This arose from the focusing of these attempts on presenting the environment as "just another part of doing business". Four emergent themes, image making, moral framing, narrative surfing, and value dissemination have been set out during this chapter in order to conceptualise this process of constructing the environment, and to illustrate how moral meaning 
with the organization was directed. These themes are considered to be important explanatory variables in understanding the cultural dynamics of green marketing in conventional organizations. However, since similar advocacy roles were not fulfilled in Flygt, little insight could be developed in relation to these themes from this case. Indeed, (at least partly) as a consequence of the absence of environmental policy entrepreneurs, far less environmental awareness, understanding and practices were made evident by respondents in Flygt rendering further data collection on these issues problematic.

Finally, and perhaps most importantly, the process of institutionalising environmental concerns into marketing activity at BTC had the effect of moral meaning being stripped from the environment, and as a consequence amoralizing green marketing. This appeared to be seen as a necessary condition for negotiating the embedded culture of the organization and realizing the policy entrepreneurs' principal goal of establishing the environment as a "normal" element in the firm's day-to-day business activities.

In the following two chapters, two rather different organizational contexts will be explored in order to provide some comparison with that presented here. These are the cases of social mission companies (following chapter) and business-NGO collaboration (chapter 7). 


\section{Social Mission Companies}

\subsection{Introduction}

Whilst the previous chapter concentrated on conventional business organizations, attention is focused in this chapter on social mission business organizations. The notion of a social mission company was broadly defined in chapter 4 and it was suggested that the main criterion for inclusion into this category was an explicit pursuit of specific social goals through economic activity. This chapter reports results relating to case studies of four such organizations: the Co-operative Wholesale Society (CWS), the Ecology Building Society (the Ecology), Beauty Without Cruelty (BWC), and the Bioregional Charcoal Company (Bioregional). In many ways these four companies can be seen to constitute a fairly diverse group. However, as it was argued in chapter 4 , the purpose of investigating social mission companies was to provide a comparative case study methodology with which to explore significantly different organizational contexts for green marketing practices. The explicit integration of social goals into the founding mission of these companies was thought to provide such a distinction.

Although it was not a deliberate sampling decision, it is significant to note that the social mission companies considered here also differed in some respects from the conventional companies in their ownership and governance status: where BTC and Flygt were wholly owned subsidiaries of joint stock companies, two of the social mission companies, CWS and the Ecology, were from the mutuality sector, one, Bioregional, was a private limited company; and one, BWC, had PLC status. In addition, the latter pair were also both founded out of 
charitable institutions, which had remained as part of the firms' governance systems. Overall then, the governance structure of the social mission companies might be regarded as providing a more favourable context in which to set their specific social goals alongside (even above) economic goals. Whilst such differences in governance structure should not be over-emphasised, they could well be important in terms of influencing the moral tone of the organization (Diacon and Ennew, 1996a). Hence, the potential role of governance is highlighted where appropriate in making cross-case comparisons.

As was the case with chapter 5 , the intention of this chapter is to explore the practice of green marketing at these companies with a view to drawing out in particular those issues considered to impinge upon notions of morality and moral meaning in some way. Clearly however, this could not be attempted without locating morality and organizational greening within the context of the companies' social missions. Indeed, given the lack of scholarly empirical research in relation to companies of this type, the study of social mission marketing presented here is considered to be an important addition to the marketing and morality literature. This then has been accorded considerable attention in addition to those issues which were also covered in chapter 5 . The format for this chapter therefore is as follows: in section 6.2 following, some background is provided on each company, along with a description of their particular missions. Following this, section 6.3 looks at how the companies have operationalised their missions, and section 6.4 looks in particular at green marketing practices. Finally, in section 6.5, the cultural dynamics of green marketing in the companies are explored in some detail, seeking in particular to focus on their moral substance and countenance. To conclude, the findings of the 
chapter are summarised, and some brief implications are considered.

\subsection{Background to the Case Studies}

Case sampling brought up at least one important disparity within the social mission companies group. One of the organizations, CWS, was clearly much larger and much longer established than the other three. Not only did this mean that a far greater volume of useful data could be collected for CWS, but also its age and size were seen to distinguish it from the rest of the group in certain important aspects. The social mission companies should not then be considered to be a wholly homogenous group, and indeed, both commonalties and differences between these cases shall also be indicated during the course of this chapter. Analysis begins with a brief description of the companies and their social missions.

\subsubsection{Co-operative Wholesale Society (CWS)}

The largest and oldest of the companies considered in this chapter, CWS was founded in Manchester in 1863. Its membership comprises of both individuals and other co-operative societies ("corporate" members). Although initially a wholesaler for the UK retail co-operative societies (widely known collectively as simply "the Co-op"), in recent years CWS has increasingly focused on retailing activities through merger with existing (often ailing) retail societies, and at the time of fieldwork was the largest single retail society in the UK. However, CWS continues to work for, and with, the other co-operative retailers through the provision of buying, marketing, distribution and other services. 
Whilst long regarded as one of the largest companies in the world, particularly from the late nineteenth century until the immediate post-war period (Bonner, 1961; Yeo, 1995), the relative decline of CWS was precipitated by the spectacular rise of the supermarket multiples in Britain (Birchall, 1994). Despite this, CWS has survived as a large and profitable business organization, currently with an annual turnover of approximately $£ 3 \mathrm{bn}, 35,000$ employees, and over 700 stores; it also has substantial dairy, agricultural, property, undertaking and other interests, as well as being the sole shareholder of the Co-operative Bank and the Co-operative Insurance Society.

\section{Social Mission}

The social mission of CWS has been enshrined in the principles of co-operation. Originally established in 1844 by the Rochdale Pioneers ${ }^{36}$ these have been set out by the International Co-operative Alliance as follows (cited in Butcher, 1996):

(i) education of the membership, staff and general public;

(ii) co-operation among co-operatives;

(iii) voluntary and open membership;

(iv) democratically based control;

(v) equitable use of profit/surplus;

(vi) limits on the participation of capital.

\footnotetext{
${ }^{36}$ The formulation, impact and implications of the original Rochdale principles have been discussed at length elsewhere (see in particular, Bonner, 1961).
} 
Beyond these basic principles, underlying social ideals have been important in guiding the movement. The Co-op biographer, Arnold Bonner (1961:292), describes Co-operative ideals thus:

An economic system based upon common ownership and mutual aid ... in which equity, individual freedom and a strong sense of fellowship would be the basis of social relations ... i.e. a system conducive to good character and consequent happiness.

Although the relative prominence of the social mission over time has been questioned, its existence in the Co-op Movement generally, and in CWS specifically, has been widely attested to in independent academic accounts (Carr-Saunders et al., 1938; Stephenson, 1963; Ostergaard and Halsey, 1965), Co-op sponsored business histories (Bailey, 1955; Bonner, 1961; Birchall, 1994; Yeo, 1995), and in the popular business press (Churchill, 1986; Thornhill, 1992; Butcher, 1996). In many ways then, the Co-op can be regarded as one of the most enduring examples of social responsibility in action. Moreover, as far back as 1857 , one of the early central figures of the Movement, George Holyoake (cited in Birchall, 1994:43), wrote of Co-op products as being morally augmented by the organization's mission:

If there are to be moral sellers, there must be moral buyers ... whether the price was high or low, the quality good or bad, they bought, because it was their duty to buy.

The corruption of the social purpose of the Movement, and in particular of the its 
largest institution, CWS, has long been an issue of concern, both for observers and members of the Movement (see Carr-Saunders et al., 1938; Ostergaard and Halsey, 1965). In particular, the devastating post-war incursions into CWS's market share at the hands of the grocery multiples has repeatedly brought into question the problems of balancing commercial needs with co-operative principles (see Bailey, 1955; Stephenson, 1963; Churchill, 1986; Thornhill, 1992; Birchall, 1994; Lee, 1997). In recent years however, CWS has attempted to revitalise its social mission and values (Birchall, 1994). The launching of a high profile "ethical banking" initiative by the (CWS owned) Co-operative Bank in 1992 was followed by a well-publicised, but substantially lower profile "responsible retailing" initiative by CWS in 1995. The latter has been portrayed both as a major corporate development in response to consumer concern surrounding ethical issues in retailing, and as a re-affirmation and modernisation of traditional co-operative values ${ }^{37}$. It was preceded by an extensive survey of 30,000 customers and members regarding their opinions on the relative importance of various social and ethical issues, and was followed by publicised commitments to initiatives relating to a) 'right to know' and customer information provision; b) animal welfare; and c) health and diet. In addition, more general promises relating to among other things, reducing environmental impacts, have also been made within the programme.

\subsubsection{Ecology Building Society (the Ecology)}

The Ecology is a mutual building society formed in 1981. It has experienced

\footnotetext{
${ }^{37}$ See for example Buckley (1995); "Responsible Retailing”, published by CWS Ltd., 1995; "Responsible Retailing: Maintaining the Co-operative Difference", video released by CWS Ltd., 1995.
} 
steady growth since its founding, and at the time of fieldwork had around 5000 investors, 400 borrowers, assets of $£ 18$ million, and 12 staff. The Ecology is a small organization, and business has been conducted entirely from a single office in West Yorkshire. Investment accounts have been operated principally via banks and through the post, and no clearing bank facilities have been provided. Recent developments in the sector have seen a number of building societies switching from mutuality to PLC status but the Ecology appears to harbour no intentions of following suit (Bibby, 1996).

\section{Social Mission}

The Ecology was formed by its original investors in order to provide finance solely for the purchase of environmentally-appropriate properties. According to company literature ${ }^{38}$, the lending policy has been as follows:

Advances shall be made to persons or on property which, in the opinion of the Board, are most likely to lead to the saving of non-renewable resources, the promotion of self-sufficiency in individuals or communities, or the most ecologically efficient use of land.

This has been interpreted broadly to include derelict property, inner-city back-tobacks, energy-efficient homes, and small-scale green businesses. Accordingly, the company has claimed that it is the only financial institution whose "sole purpose is to seek ecological and environmental gain through its lending

\footnotetext{
${ }^{38}$ Source: "Ecology in Action" (pamphlet), Ecology Building Society, July 1994.
} 
policies" $^{\text {,39 }}$. Its mission then has focused explicitly on environmental concerns in relation to property, and potential investments have been rated according to their environmental attributes. To this end, guidelines have been drafted by the board so as to set out green lending criteria ${ }^{40}$. Hence projects can be distinguished between those that are "inherently ecological" and those that are "potentially ecological". Environmental criteria have also been set out in order to rate them as projects the Ecology is either "keen" to lend on, "prepared" to lend on, and those that are "marginal". However company respondents suggested that such ratings could be problematic, and that progress in this direction had been fairly slow. Nonetheless it does indicate that environmental as well as financial criteria have been central to the company's lending policies.

\subsubsection{Beauty Without Cruelty (BWC)}

BWC was founded in Kent in 1963 by the trustees of Beauty Without Cruelty Charitable Trust to manufacture and distribute "vegetarian" cosmetics. Undercapitalization and limited growth in the 1960s and 1970s led to insolvency, and by the mid-1970s BWC was close to bankruptcy ${ }^{41}$. Turnaround began with the appointment of a new Managing Director in 1977, followed by financial restructuring leading to PLC status and independence from the charity in 1980 .

Following re-organization, the company's fortunes improved substantially and

\footnotetext{
${ }^{39}$ Source: "Ecology in Action" (folder), Ecology Building Society (undated).

40 "Ecology Building Society - Lending Guidelines", draft document prepared by Ecology Building Society, 1996.

${ }^{41}$ Source: "Thirty Years of Beauty Without Cruelty Cosmetics: A Profile and Short History of Beauty Without Cruelty PLC", BWC, 1993.
} 
although still a tiny player in the cosmetics industry, BWC has established itself as a viable concern. Initially concentrating on mail order business, distribution was expanded into the health food and chemist trade in the 1980s, followed by in-roads into the retail multiples in the early 1990s. However, recession in the 1990s hit the company hard and the decline of business with the retail multiples provoked substantial redundancies in the company. By 1996, BWC had 43 employees and operated a turnover of $£ 1.5 \mathrm{~m}$ p.a. It operated through three sites: a head office and retail outlet (first opened in September 1995), both in Kent, and a factory in Cumbria.

\section{Social Mission}

Having been founded by an animal welfare charity, the social mission of BWC has been to provide cosmetics within the vegetarian ethic. This has involved the avoidance of all animal ingredients other than those harmlessly obtained (and all slaughterhouse or fish industry by-product), and a refusal to use animals in testing, or use ingredients specifically tested on animals. Its mission has been defined as follows ${ }^{42}$ :

1. To set an example to the cosmetics industry by not testing on animals and, more importantly in the current context, to discourage the use of new ingredients through our strong Fixed Cut-Off Date criterion of 1976.

2. To leave a fund to help animals through the eventual disposal of its shares.

42 ibid. 
3. To provide cosmetics of the finest possible quality at very reasonable prices for people who care about animal welfare.

Having described itself variously as a "vegetarian" company or one "dedicated to animal welfare", BWC has claimed that all its directors and shareholders are animal welfarists and vegetarians ${ }^{43}$, to some extent aligning its governance system with the rest of the social mission companies. Equally, it has stated publicly that its ethical mission is primary and that products that can not be produced within its ethical parameters will not be manufactured ${ }^{44}$. Its mission then could be described as environmental - but only in the sense that animal welfare is an environmental issue - since other green issues have not been explicitly referred to in its mission. Indeed, the chief executive set out the company's priorities thus: "we care about the environment but we put animal welfare foremost". In this sense, BWC might be regarded as having the most narrowly-defined mission of the companies considered in this chapter.

In the $1990 \mathrm{~s}$, the anti-testing policy of the company became particularly prominent, highlighted in the main by its strong advocacy of the Fixed Cut-Off Date criterion. This states that only ingredients in general use and accepted as harmless before September 1976 (when the EU Cosmetics Directive was published) can be used in products; all new ingredients are rejected on the basis that they are required by law to be tested on animals by third parties. This focus on animal testing can be explained by the high public profile of the issue and also by the prevalence in the marketplace of non-tested claims by BWC's

\footnotetext{
${ }^{43}$ ibid.

${ }^{44}$ Source: “The Policies of Beauty Without Cruelty”, BWC, August 1995.
} 
competitors. Where these companies have not adhered to the fixed cut-off date criterion, company executives accuse them as having "betrayed the whole campaign" by misleading consumers.

\subsubsection{Bioregional Charcoal Company (Bioregional)}

The newest and smallest of the companies considered in this section, Bioregional was founded in January 1995 by the Bioregional Development Group (BDG) - an environmental charity centred on promoting bioregionalism, i.e. local production for local needs. The company is essentially a broker for charcoal products, providing a co-ordinating mechanism between local woodburners and national retailers; it also provides marketing support and quality assurance. At the time of fieldwork, the company was situated in a single office in Surrey, and was ran on an informal and virtually voluntary basis by its two directors, both of whom continued to hold positions outside the company (one in $\mathrm{BDG})$. Turnover was in the region of $£ 40,000 \mathrm{pa}$.

\section{Social Mission}

The mission of Bioregional is also fairly specific in that the company has been established principally to promote the use of environmentally appropriate and decentralised systems of production and distribution for charcoal products. However, this mission also encompasses issues such as job creation and sustainable land use. Charcoal production utilises a technique known as 'coppicing' which involves taking a sustainable harvest from local woodland trees (to be burnt for charcoal) in such a way as to create suitable habitats to encourage biodiversity of fauna. According to its directors, the purpose of 
Bioregional has also been to set an example to an otherwise sceptical industry that such social and environmental goals could be achieved within a commercial context.

\subsection{Marketing Strategies}

The previous section sought to describe the particular social missions of the four sampled companies. In this section, the intention is to identify how the firms have attempted to operationalize these missions within their marketing strategies. In particular, important questions arise as to the strategic goals of the organizations in terms of balancing profits and principles. Whilst the concepts of social marketing and societal marketing introduced in chapter 2 (see 2.2.3) were useful in terms of analysing the responses of respondents in the social mission companies, neither seemed to provide a full conceptualisation of the situation that was revealed. Analysis in fact revealed that the companies' adoption of a particular marketing strategy could be broadly described in terms of two key constructs - campaigning priorities and mission positioning - which encapsulated elements of both existing concepts. Therefore, using insights from societal and social marketing, these two themes shall be considered in detail below before bringing them together to conceptualise the different marketing strategies of the social mission companies.

\subsubsection{Campaigning Priorities}

One of the key features of the social mission companies which distinguished them from conventional companies such as those considered in chapter 5 was the importance attached by the firm's leaders to campaigning for social and 
environmental change of some kind. Senior executives articulated a common goal of converting external publics to their world-view; and whilst this was also true to some (lesser) extent of the companies considered in chapter 5 (e.g. through political lobbying and school information packs), in the social mission companies this was portrayed as more than simply a benefit for the company, but as an end in itself. Clearly, the system of governance in the company was important here, and the collusion of members/owners was essential in allowing the company to be used as a vehicle for social change. A useful approach to understanding this construct is thus provided by Kotler and Zaltman's (1971) notion of social marketing. Although originally formulated in relation to nonbusiness organizations, Kotler and Zaltman's definition (p.5) reflects very much the practice of the social mission companies:

The design, implementation and control of programs calculated to influence the acceptability of social ideas and involving considerations of product planning, pricing, communication, distribution, and marketing research.

According to Kotler and Zaltman, the core product offering within social marketing is the cause itself. This is made tangible by the supply of products and services which advance the social cause. In the four sampled companies, such linkages between cause and product were certainly evident, although not always to the same degree. Hence, Bioregional's mission to promote bioregionalism was made tangible by charcoal products; CWS's mission to promote fairness, equality and democracy was made tangible through the provision of retail services; and so on. However, senior executives from the social mission companies clearly differed in the relative marketing emphasis which they 
applied to promoting (intangible) cause and (relatively tangible) product.

In general, executives saw the basic role of their companies in effecting social change as simply information providers, e.g. through customer leaflets, newsletters, reports, publicity, etc. Moreover, the material existence of the firms in the market was also presented as an important indirect influence on society: first, because it provided an example to government and industry that social and environmental goals could be achieved in a commercial context; and second, because it enabled the provision of social or environmental products that would not otherwise be available to consumers. The companies could however also be seen to adopt more direct campaigning tactics such as political lobbying, educational visits and materials for schools and colleges, public relations activity, supplier seminars, etc. However, these were not uniformly applied, and there were clear differences in the priority accorded to campaigning as a company activity. It was not so much that the firms' leaders were necessarily confronted with the option of pursuing profit or principle, but more a question of resolving why the firm was in existence in the first place. Was its foremost goal to promote social change as Kotler and Zaltman (1971) suggest in the case of social marketing? Or was it to provide a socially acceptable product, and in so doing promote social change indirectly, as might more befit a societal marketing orientation? This can be regarded as a question of organizational purpose (Freeman and Gilbert, 1988).

Broadly speaking, the companies could be classified into two categories: those that viewed campaigning as a primary activity (BWC and Bioregional) and those that viewed it as a secondary activity (CWS and the Ecology). The principal 
difference here was the balancing of resources between, on the one hand, furthering the firm's economic success, and on the other, promoting social and environmental change. The 'primary' group clearly laid more importance on the latter than did the 'secondary' group. Indeed, for the 'primary' group, campaigning on the part of company leaders was shown to take up a substantial amount of their time; moreover, it was acknowledged that economic success had to take a subordinate role to the firm's social mission. The chief executive of BWC for example argued:

The goals of being ethical and maximising profit and growth are incompatible ... for us the balance is always towards animal welfare.

In the case of Bioregional, this had even had the extreme consequence of its directors forgoing remuneration in order to ensure the survival of the firm with its principles intact.

For the 'secondary' group companies, campaigning that was not directly related to firm performance was principally seen as something that should only be undertaken once sufficient resources had been accumulated:

It hasn't really been appropriate for us ... perhaps we'll attempt more of a campaigning role now that we have the resources... but at the end of the day it's not what we are here for.

Equally, campaigning would be resourced after a certain degree of profitability had been attained, or as one executive from CWS put it, "between what I call 
the gross, net profit and the net, net profit." Hence, it is not so much that these firms would have sacrificed their principles in pursuit of profit, but they were likely to spend less time and resources on actually pushing forward certain social and environmental issues should profitability be threatened. Social marketing then would be principally centred around indirect campaigning tactics and PR activity.

Significantly, these categorisations were not regarded as totally static. The firm's campaigning priorities appeared to be a direct consequence of the personal inclination of its leadership. In the Ecology for example, it was related how one of the original founders of the company had departed acrimoniously due to a refusal by the rest of the board to adopt a more substantial campaigning profile. A more agreeable reaction may have precipitated an entirely different social marketing strategy, although as the current chief executive warned, "if we'd gone down the campaigning route, we wouldn't be here now." Also, priorities clearly altered over time and several respondents in the social mission companies described actual or potential developments in their firm's social marketing strategy. Nonetheless, whilst there was some recognition that growth and economic success provided opportunities for increased attention to campaigning, there was also evidence of concern over the potential transformation in priorities that such a commitment to growth might bring. Hence, the balancing of social marketing objectives and firm performance objectives was a constant issue for consideration for the social mission companies' leadership, and the priority accorded to each could be seen as a central defining feature of the operationalization of their missions. 


\subsubsection{Mission Positioning}

The campaigning priorities construct is associated with the company's overall social marketing stance. This second construct, mission positioning, relates to how the social mission of the company had been incorporated within the selling proposition for their products and services. Whilst the main issue of concern here is the environment, other social and moral issues were clearly integral to the complete bundle of concerns marketed by the companies. Although not always presenting a coherent approach to the treatment of potential issues within marketing activities ${ }^{45}$, clear differences could be discerned amongst the social mission companies regarding the prominence given to corporate ethics or mission in constructing product marketing strategies and in shaping and colouring particular marketing tactics.

A useful approach to this question is provided by Kotler's (1972) notion of societal marketing which emphasises the combined marketing goals of profitability, customer satisfaction and long-run consumer welfare. Kotler's (1972) view of societal marketing articulates a mixture of moral and self-interest motivations on the part of practising companies, with the latter articulated mainly in relation to avoiding regulation. In looking at green marketing though, Prothero (1990) sought to re-frame the self-interest aspect of societal marketing by arguing that the orientation itself - in this case, the social mission of the organization - might provide positive product augmentation. Social mission

\footnotetext{
${ }^{45}$ BWC management for example repeatedly stressed the importance of accurate and fair (as opposed to simply legal) marketing claims in relation to animal testing but continued to use the relatively ambiguous (and much criticized) term "environmentally friendly" on company paper and packaging.
} 
company managers here indicated a range of positioning platforms for their products from those where augmentation of the mission was central to positioning strategies, those where mission augmentation was prominent but not over-riding other positioning variables, and those where mission augmentation was marginal. These are considered below.

\section{Mission Central}

Of the four social mission companies, the Ecology could clearly be seen to be making the strongest attempt to position its products purely, or at least principally, on the basis of the augmentation afforded by its mission. Its core product offering of investment and lending services was focused entirely on ecologically appropriate properties and as such was believed by managers to appeal principally to a niche of green consumers. According to executives, these were likely to be fairly involved green consumers, perhaps members of environmental organizations such as Greenpeace or Friends of the Earth, even (to some extent undesirable) "dreamy" types with more environmental than financial concern. Correspondingly, investment services had traditionally been priced at a green premium (although this had gradually been reduced in line with the Ecology's growth), and print advertisements were primarily targeted in environmentally-oriented media. Nonetheless, it was suggested by the chief executive that the Ecology had increasingly been making an attempt to appeal to a wider range of potential customers in order to communicate its environmental message outside of its traditional niche and thus avoid simply "preaching to the converted". 


\section{Mission Prominent}

Occupying a more moderate approach, but still positioning itself mainly in relation to the augmentation afforded by its mission and its ethical stance, BWC could be said to utilise a mission prominent approach. Respondents regarded their customer base as fairly diverse and likely to extend well beyond the 'green niche'. Equally, it was emphasised that products were competitive on price and quality criteria as well as offering a non-tested alternative to mainstream cosmetics. Indeed, the chief executive claimed that the charging of an 'ethical' or 'green' premium was not only avoided by the company, but would be "morally questionable" as well. Hence prices were kept to the minimum dictated by costs.

Marketing communications also reflected a mission prominent positioning stance. Print adverts and point-of-sale materials had long emphasised the social mission of the company, although other tangible and intangible attributes such as price and quality had increasingly been stressed as well. Its 1996 poster campaign for example carried the following copy:

\section{Natural Cosmetics}

Quality - Performance - Fair Prices

Created for people who care about the earth and the animals

\section{Mission Marginal}

The remaining two organizations could be regarded as having a more marginal utilisation of the augmentation afforded by their social missions as a positioning variable. Bioregional was regarded by its directors as having moved away from 
an explicitly environmental marketing platform to a more conventional approach which stressed quality, value for money and "Britishness", thus effectively "hiding" the social and environmental issues in question. This had occurred as a result of the failure to secure sales on the environmental platform, thus prompting a change which was seen as more appealing to their target market of retailers. As one director explained:

I suddenly realised that we were giving them all this stuff about the environment, and its quite a complex story to talk about ... and now I realise that they're going: Shit! I don't want to get involved in this! Its a disaster! .... So now we don't mention anything about anything like that. We just say, we can provide you with $x$ amount of British charcoal; it will be packed however you like; this is the price; the quality is controlled; it will be environmentally certified.

CWS also could be seen to make only marginal use of its ethical stance in establishing its market positioning. However in contrast to Bioregional, CWS appeared to be gradually increasing the role of its social mission through the newly launched "responsible retailer" initiative. Whilst this was seen as important in terms of creating some kind of differential advantage should other factors be equal, it was not however seen as ever likely to supersede conventional positioning variables of price, quality and store location. This was explained by executives in terms of the limited size of the 'ethical consumer' niche since the company's retail outlets had to appeal to a much wider consumer base within the catchment area in order to be commercially viable. Hence it was a matter of, "appealing to the majority not the minority". Ethical issues were thus considered to be appropriate, but not central, to the appeal of the company: 
Nobody is ever going to shop with us because we are nice people... maybe people will feel warmer about shopping with us, but that wont be the reason why they make the decision to shop in the first place.

\subsubsection{Strategies for Social Mission Marketing}

In general then, there appeared to be some recognition within the social mission companies that augmentation by 'good ethics' alone would rarely constitute an effective marketing platform, unless a relatively small green niche market could be identified and targeted profitably. Clearly with a positioning platform either wholly or prominently based on the augmentation provided by their social mission, social marketing objectives could be realized more effectively than with a marginal stance since the company's mission itself would be a substantial part of the product offering. However, targeting the majority of consumers outside of the green or ethical niche could be seen as providing other advantages since a) it may lead to greater profitability, thus releasing greater resources for more effective campaigning outside of product marketing activity; and b) it may provide potential for communicating the company's mission to an audience as yet 'unconverted' to the cause. Clearly then, the decision was likely to be contingent on the type of product concerned, the characteristics of the market, and the strengths and priorities of the company and its leadership.

These two constructs - campaigning priorities and mission positioning - are considered to be fundamental to explaining the overall marketing strategies adopted by the social mission companies. Taken together, they provide a framework with which to categorise the possible routes through which the mission can be carried forward to various audiences and in various contexts. In 


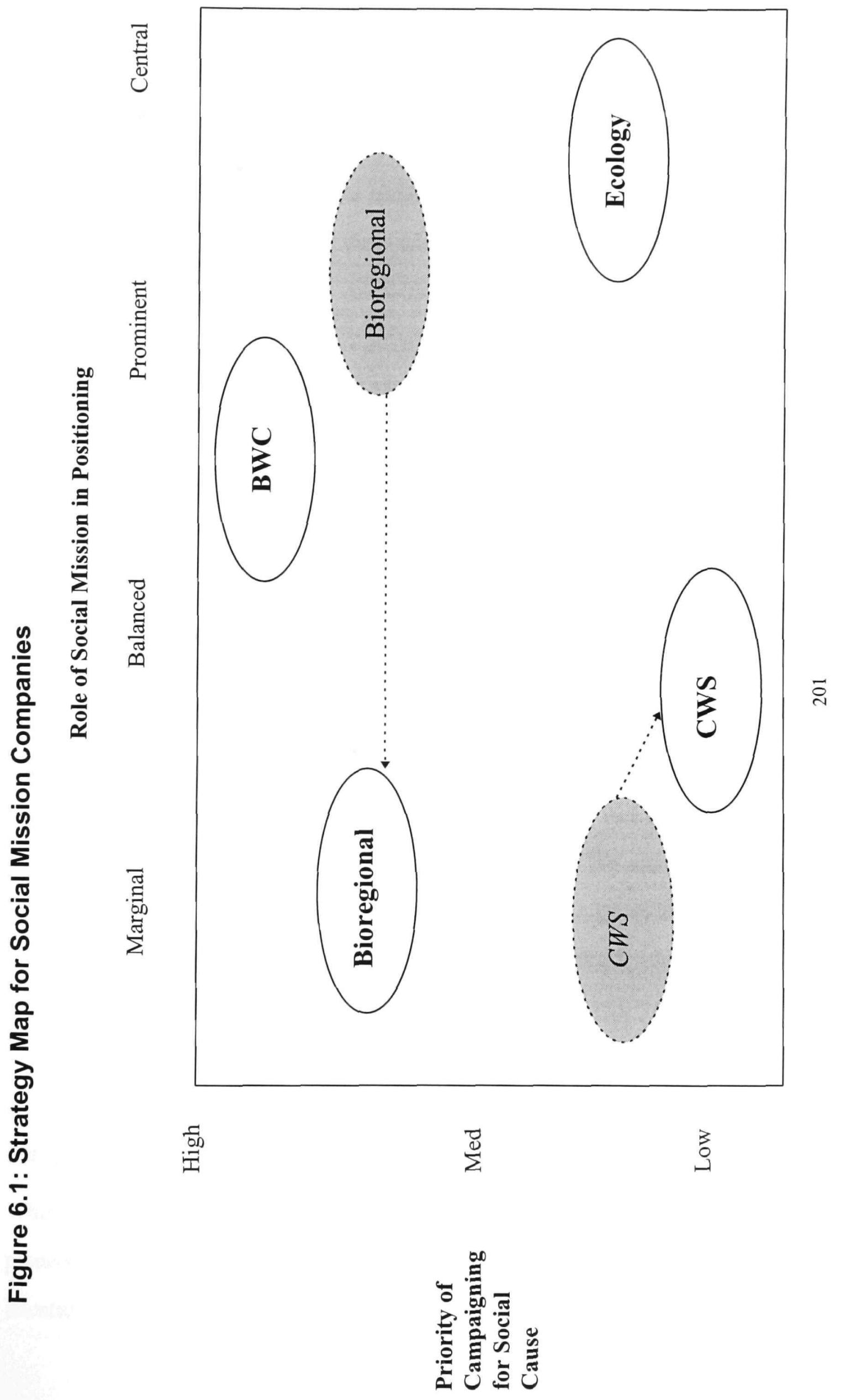


this way, greater analytical and explanatory power can be added to the basic assumptions provided by the social and societal marketing concepts. Hence, the four sampled organizations, and other similar companies, can be usefully plotted according to their 'scores' against these constructs. This can be seen in figure 6.1 .

Clearly then there are a number of ways in which marketing strategies can incorporate social objectives for social mission companies. Although obviously the top right hand corner position represents the most direct approach, and the bottom left the least, it should not be assumed that any particular position is 'better' or 'more ethical' than others: they are simply different approaches to the same problem. It is also important to remember that there is nothing to prevent movement within the grid, and indeed some change should be expected given shifts in the market and changes in the goals of senior executives and owners over time. Changes in strategic positioning are represented in figure 6.1 by dotted lines, with the previous position being indicated in grey. However, having set out here the basic organizational purpose and strategy of the social mission companies, this should provide the broad context in which to go on and set out the tactical and cultural approaches to green marketing revealed by the study. It is to these issues that the chapter now turns.

\subsection{Green Marketing}

Whilst to some extent the social mission companies could be regarded as pioneers of green marketing in their sectors, they exhibited varying degrees of attention to environmental issues: for the Ecology and Bioregional it was their 
main focus; for BWC, animal welfare took precedence over other environmental issues; and in CWS the environment was considered a secondary social concern behind issues such as animal welfare, consumer information/labelling, and store access. Such differences were mirrored in the attention accorded to products' environmental performance and hence the green strategy adopted by the organization. Thus, in the Ecology and Bioregional there was an attempt to provide the most radical environmental attributes on the market, suggesting an approach akin to Peattie and Ratnayaka's (1992) "greening the company" strategy; in BWC managers claimed that environmental issues were largely "out of our control', indicating that environmental considerations may not have been fully explored in product development reflecting a "piecemeal" strategy ${ }^{46}$; and in CWS managers highlighted the importance of offering consumers a wide choice of products (to a certain extent irrespective of their green attributes) and consequently green product and service developments were broadly in line with conventional retailer best practice (such as BTC in the previous chapter) - a "follow the herd" strategy.

Product quality was not presented as a problem by the social mission company managers in relation to their own offerings, although it was recognised that it had been in other product categories, leading to a lack of market success for many green products. Interestingly, similar examples of this phenomenon were presented here as they had been at BTC, cleaning products such as washing powder and washing-up liquid again being repeatedly cited. Hence, managers

\footnotetext{
${ }^{46}$ Results were consistent with Prothero and McDonagh's (1992) findings in their study of the UK cosmetics industry where cruelty-free companies were shown to pay less attention to environmental attributes than did conventional cosmetics companies.
} 
had recognised that consumer perceptions of potential performance shortfalls might have been an important barrier to trial and adoption for their own products - particularly in the light of competition from more conventional companies in green product markets. As a result, products had been increasingly positioned as value-for-money, quality alternatives to non-green offerings. In respect to Kotler's (1972) typology of societal products then, they could be regarded as having moved from focusing on the "salutary" aspects of products, towards considering also the more immediate satisfactions of "desirable" products (see table 2.1, p.36).

In terms of price, senior executives in the three smaller companies - BWC, Bioregional and the Ecology - generally felt that they were at a disadvantage compared to their more conventional rivals. This was due either to the limitations imposed by their small size or by their commitment to their mission (entailing higher cost processes/components), or both. There was no indication in any of the social mission companies however that prices had been raised artificially to reap a green premium from concerned consumers. Respondents generally argued that prices were set at a fair level, sufficient to maintain survival - an issue of some concern for two of the companies. Despite many green products being new market entrants then, penetration pricing to encourage trial did not seem to have been considered as a viable option. However, where the avoidance of penetration pricing in conventional companies might be due to the marginalisation of green products in firm's marketing plans (Wong et al., 1996), in social mission companies it might be more readily explained by either lack of resources per se or by the allocation of resources to social campaigning activities. 
For at least two of the social mission companies (BWC and Bioregional), distribution had remained their most critical marketing issue. The necessity of securing retail space had made them reliant on establishing supply contracts with large retail organizations. However, managers described this as highly problematic, since although retailers were not portrayed as especially hostile towards 'green' or 'ethical' products per se, their buyers were depicted as fairly ruthless towards small or new suppliers compared to larger, incumbent ones. Hence, managers identified a dire need for a far greater level of support and commitment from retailers for 'ethical' product suppliers.

These problems had provoked different responses. Bioregional had moved from a mission prominent positioning strategy to a marginal one, increasingly highlighting conventional product attributes above environmental ones in order to appeal more directly to the "hard-nosed" and "conservative" retailer buyers. Conversely, BWC - which in the recent past had made substantial redundancies due to the sudden loss of contracts with retailers - had moved into retailing itself, thus avoiding contact with the "fickle" retail multiples 47 .

Such distribution problems could not be easily offset by communications and promotions policies due to the constraints of limited marketing resources and campaigning priorities. Hence, much of the promotional activity for the social mission companies was focused on public relations and publicity. Whilst interest

\footnotetext{
${ }^{47}$ At the time of the fieldwork, this was a very new development and amounted only to a single experimental store, the success of which could not at the time be determined with any certainty.
} 
generated from their social missions appeared to have engendered considerable, and for their size, disproportionate success through these elements of the promotions mix, several managers expressed concern over the limited effect this appeared to have had on sales. This prompted at least one manager to lament: "we are the brand virtually everybody has heard of and nobody has ever seen!" It was also stressed how this positive publicity had alerted competitors and consumers to the issues of concern, subsequently helping the industry as a whole rather than aiding them in particular in securing distribution deals ${ }^{48}$.

The environmental content of promotional messages obviously reflected positioning strategies. Interestingly though, all companies in at least some of their environmentally-themed communications could be seen to have stressed a long and consistent history of social and/or environmental concern, often by emphasising the firm's year of founding. To some extent this parallels similar communications at BTC as revealed in the previous chapter, and here also it can be seen to be an attempt to present a picture of success and stability in what might otherwise be regarded as a radical and unstable area. Moreover, it reflected a clear attempt by individuals within the social mission companies to distance their company from those of their competitors whom they regularly accused of having simply (even cynically) "jumped on the bandwagon" in recent years without attendant moral values and convictions.

\footnotetext{
${ }^{48}$ This suggests that the introduction of 'new' social and environmental issues into company activities might have the effect of rendering communications activities as 'pioneer promotion' for the issue, rather than promoting the company's particular approach to it. First mover advantages seemed to be limited, especially in the context of relatively conservative industrial consumers.
} 
Also in line with findings in chapter 5, managers stressed the negative impact that misleading green claims by other companies had had on their own potential to make meaningful claims. Accordingly, it was related how they had attempted to describe the complex reality of particular issues without resorting to bland slogans. This was seen as requiring an information-led approach involving the presentation of quite extensive descriptions of company policies or environmental practices in marketing communications. The social marketing priorities of the companies had seemed to elicit a perception that final consumers needed to be informed of the complexities of moral issues relating to particular products, especially given perceived knowledge gaps regarding the relative value of competing offerings. Indeed, some managers saw this as an issue critical to their success, since concerned customers were seen as being (mistakenly) convinced by less radical offerings from competitors on the belief that these environmentally labelled products were equivalent in environmental terms.

\subsubsection{Green Marketing and Social Responsibility}

Green marketing in the social mission companies was then in some ways more developed than in the conventional companies considered previously, but certainly not universally so. However, underlying these greening initiatives were justifications quite different from the "sound business sense" rationales of executives in the conventional companies. Indeed, managers' rationalizations tended to steer away from instrumental notions of enlightened self-interest towards more explicitly moral notions of social responsibility. This would represent a perspective on green marketing more towards the 'deep' green end of the spectrum. Indeed, executives were on the whole pessimistic about the prospects for consumer pressure in establishing viable green markets in anything 
except limited niches. Hence green marketing was often portrayed as emerging out of a drive from within the organization, usually from board level, rather than in response to consumer pressure:

All the studies show there is concern out there, but that doesn't translate into people voting with their pockets. They will say they are concerned ... but its not something you can build a business on easily in mainstream markets. There are only small niche markets where you can build your company on the environmental friendliness of your product .... and I see the future in terms of corporate responsibility, the company saying we will only stock environmentally friendly products.

Interestingly, among the social mission companies, the strength of managers' claims for this internal drive did not correlate with their companies' positioning policies as might have been expected. Hence, even where consumers' high concern for ethical or environmental issues had prompted mission-focused positioning by the company (such as in the Ecology), an internal cultural impetus was still asserted to be the principal driving force for greening. Moreover, managers also tended to claim that they would not reduce the environmental performance of their products even should customer demand for such green attributes be lacking. In Bioregional for example, the lack of customer interest had caused them to play down and marginalise the environmental dimension of their selling proposition (hence, another example of muted greening), but it had not led to any change in the environmental performance of their products. 
Overall then, whereas these companies were clearly incorporating considerations of long term consumer and societal benefit as befits a societal marketing orientation, in most circumstances this concern with long-term consumer welfare originated from the moral principles of the firm's leaders rather than as a selfinterested response to external pressures. This represents a considerably more explicit moral perspective on the SMC than is presented in the literature (e.g. Kotler, 1972; Abratt and Sacks, 1989; Prothero, 1990). Moreover, the assessment of which products might be in the consumer's and society's longterm interests were predominantly based on the presumptions of the firm's leadership rather than "by all the individuals who together constitute a society" as has been somewhat ambiguously suggested by Abratt and Sacks in their reading of the SMC (1989:33). The exception to this was at CWS where managers claimed that the company's social and ethical policies should directly reflect those concerns identified by market research and environment scanning. Hence, "we have to take the stand looking at what our customers are concerned about"... "we have to take our lead from what the pressure groups ... (and) the media (are) going to be interested in." Critically however, the environment had not been rated as significant as, say, animal welfare, and was therefore seen as a "middling type of issue". Accordingly, customers should not be "forced" or "nannied" into making green choices, since they were seen as largely unwilling to adopt green purchasing behaviour. Hence, in contrast to the smaller social mission companies, managers in CWS argued that giving customers the opportunity to make informed choices, rather than making those choices for them, was the socially responsible role for a retailer in competitive markets since this protected and enhanced consumer sovereignty (Smith, 1990; 1995): 
Rather than us making the choice for the customer and saying you are not going to have that product, we've said: it's there, but that one is also there, and you must understand the consequences of purchasing that. In a way, trying to educate the customer - and leaning towards taking the responsible side - in a way that is trying to strike a balance.

\subsection{Cultural Dynamics of Green Marketing}

Clearly then, although different marketing strategies had led to differing degrees of societal marketing orientation, notions of morality were integral to some extent to what and how the social mission companies had attempted to accomplish in green marketing terms. This does not mean that they were necessarily ethical per se, or even more ethical than the conventional companies, but it does suggest that the firms' goals and purposes were more fused with moral concerns than for those in the previous chapter. The question to which this chapter shall now turn then is: how does this manifest itself inside the organization? If organizational greening is explicitly linked to moral concerns, does this mean that the culture of the organization will be more suffused with a discourse of morality than if it is not?

Managers claimed that the drive for greening in their companies was generally derived from a cultural impulse from within, mediated in some circumstances by consumer preferences. However, in all of the companies, the social mission tended to be associated with particular individuals or sections whose role it was to manage and interpret the mission in a meaningful way. For these individuals the environment, and indeed moral issues generally, were seen as the daily currency of their workplace roles. Again, they might reasonably be referred to as 
policy entrepreneurs. This section identifies and describes the role of such policy entrepreneurs in the social mission companies, and goes on to reveal the implications that their behaviour might have for moral meaning within the cultural knowledge of the organization.

\subsubsection{Policy Entrepreneurs}

Overall, the environmental agenda in the social mission companies tended to materialise in much the same way: that is, centrally and at senior levels. Policy entrepreneurs were located principally in very senior management positions: in the three smaller companies this was usually at board level; in CWS, in the corporate marketing division ${ }^{49}$. It was suggested that such seniority was necessary for successful environmental marketing projects to be initiated:

If the people at the top think it's important to be environmentally friendly then it will filter down from above. You can never push it upwards. The belief has to come from the top to really get the response from it.

Equally, policy entrepreneurs were to be found at senior levels in the three smaller social mission companies because these managers had frequently joined or founded the company in the first place from a sense of personal moral conviction: "I like to do my own little bit for the environment" ... "I have come into this from a sense of responsibility rather than anything else". In CWS this was not found to be the case, and reasons for adopting the role were broadly in

\footnotetext{
${ }^{49}$ Given the size of CWS, other policy entrepreneurs were likely to have been present elsewhere in the organization. However, these were not identified during fieldwork.
} 
line with those revealed in chapter 5 .

In contrast however to the policy entrepreneurs revealed in chapter 5 , those in the social mission companies did not need to utilise political skills in securing support for environmental marketing projects since they already held positions with sufficient power to impose green initiatives upon their organizations. Moreover, they appeared to place considerably less emphasis on the importance of involving others within the organization. In general, environmental issues were managed centrally so that shop floor employees had little involvement with the environment and could, as a consequence, defer responsibility to their managers. Hence, the possibility of front line employees either contaminating the company's carefully conceived environmental policy, or of mis-informing customers in a complex area, was significantly diminished. In CWS for example, customers were encouraged to use a free-call information line rather than approach store staff for information on social and environmental issues. This connected them directly to head office where customer relations staff could respond from pre-prepared scripts based on the company's 50-plus page "Viewpoint" manual which set out the company's position on a range of community, environmental and customer issues.

\subsubsection{Constructing the Environment}

Given their relatively un-contested positions as the principal environmental reality definers within the social mission companies, policy entrepreneurs were fairly free to construct the environment in whatever way they wished. In Bioregional and the Ecology, a single site, small workforce, and focused environmental mission meant that the leaders' concept of the environment was 
effectively also the firm's. Indeed, these managers could command knowledge of, and interest in, sophisticated environmental concepts and arguments outside of the more conventional concerns of recycling and energy conservation characteristic of the individuals described in chapter 5. This included reference being made to issues of biodiversity, sustainable living, animal rights, selfsufficiency, decentralisation and social justice, among others. Such a thorough construction of the environment left little room for opposing discourses in the firm.

In BWC this was less the case due to a mission more centred on animal welfare than the environment. Consequently, the environment as a concept tended to be overwhelmed by animal welfare concerns, leaving little room for other issues to surface. Also, the need to bridge separate physical locations meant that the environmental agenda tended to be left to the personal discretion of individual managers to define as they saw fit. Whilst this provided some scope for environmental policy entrepreneurship, the environment tended to be constructed very much in terms of the practicalities of the immediate location:

[Policy entrepreneur A] is always picking up boxes and taking them off somewhere. She re-uses everything .... but I don't like all that mess. I prefer the place clear, you know. So I tend to just throw them out.

Hence, whilst phrases of the kind, "we do what we can", were commonplace in BWC, the was little evidence of a unitary environmental agenda as such.

In CWS, a unitary and sophisticated environmental discourse was beginning to 
emerge, although it was not fully developed when fieldwork took place. Given the need to communicate environmental issues to external publics, the corporate marketing function had assumed responsibility for defining and communicating CWS's environmental policies and programmes. Understandably, this had resulted in the environment being incorporated within the pantheon of "consumer issues", which included among other things, animal welfare, store access, bullying, nutrition, food hygiene, and customer relations generally. Hence, the environment tended to be depicted as something customers either wanted or did not want, could or could not afford. Indeed, the culture of CWS appeared saturated with at least the rhetoric of consumer sovereignty, and few assertions were made without appeal to the exigency of customer needs and demands ${ }^{50}$. Other conceptions of the environment therefore tended to be relegated as a consequence. As one operations manager explained it, consumer focus remained the single most important issue in securing commitment and support for environment-related projects:

When you're selling initiatives to other staff, the one you put first is the customer, because its good for the customers. That's always number one. This is after all a retail company. After that comes the commercial justifications - the cost savings ... and only after that you look at the environmental side ... because at the end of the day, saving the world is not going to keep us in business.

Evidently, the cultural hegemony of the corporate marketing function within CWS had meant that it was here that the doctrine of "consumerist" green

\footnotetext{
${ }^{50}$ This was the case throughout the organizational departments and hierarchical levels that the researcher had direct contact with, and not only in marketing-related departments.
} 
understandings tended to be originated and ossified. For the individuals in the section who had been cast into the policy entrepreneur role, the constructing of environmental meanings within the marketing discourse was not significantly difficult given the lack of an existing frame of reference for the environment. Indeed, to some extent the environment as a concept had remained equivocal and open to localised interpretations. This had generated little consensus over what constituted the environment within the company, and many CWS representatives continued to rely on working definitions which could practicably be fashioned to suit the requirements of a given situation. Indeed, even the incorporation of the environment within the rubric of "consumer issues" had tended to produce a blurring of social, ethical and environmental issues for many managers and staff, rendering the boundaries of the environment indistinct ${ }^{51}$.

The notion of the environment as a consumer issue was also cemented more firmly by presenting it as something of a return to traditional (and to some extend side-lined or forgotten) co-operative values which focused explicitly on fair trading and consumer welfare. Thus, in much the same way as executives in BTC had surfed appropriate corporate narratives in order to gain acceptance of green marketing activities, the environment in the CWS had been attached to existing corporate narratives of consumer choice and welfare (although, as shall be shown in section 6.5 .4 , a similar dexterity with narratives was also manifested in other ways among the social mission companies).

\footnotetext{
51 For example, discussions with interviewees on the subject of green marketing and management tended to be steered by the respondent to encompass a whole range of social, ethical and general consumer issues; as far as the interviewees were concerned, the interviewer could set any necessary definitional boundaries between them.
} 


\subsubsection{Moral Framing}

Clearly this hierarchical and quite centralised origin of environmental meanings in the social mission companies had significant influence on the sense and form of morality attached to green marketing activities. Since it was senior level respondents who constructed environmental meanings, it was they who also set them within a particular, and usually quite explicit, moral context. Indeed, many senior respondents were highly adept at incorporating (at least some) environmental issues within quite sophisticated ethical arguments, and in the main it was depicted as one of a range of, or even the central, moral responsibility of the firm. Obviously this was driven by, and indeed reflected, the particular mission of the company. However, it was also apparent that environmental concern was regarded more as an individual moral responsibility: for the firms' leaders in the small social mission companies; for the customer in CWS. These positions are illustrated below.

In the three smaller social mission companies, board level executives tended to speak of their own moral beliefs and convictions on environmental issues, reflecting to a greater extent, a background in active environmentalism of one sort or another. Thus, the founders and CEO of the Ecology had been members of the Ecology Party, the founders and CEO of BWC had been involved in animal welfare work, and of the chairmen of Bioregional, one had run the local Greenpeace group and the other had been pivotal in setting up the BDG Charity. Just as these managers were clearly conversant with quite sophisticated environmental concepts, so too were they found to use distinctly moral and emotional vocabularies in relation to environmental issues. This was illustrated with phrases such as "it was furthering my own beliefs"... "I think it is morally 
wrong to torture other creatures in order to seek a benefit for ourselves"..."I've always been environmental'. The environment was thus conceived as being incorporated within a personal moral belief system rather than a collective one.

Staff in CWS also tended to regard environmental concern as a matter of individual conviction, with statements such as, the environment is very individual" ... "commitment is very localised" being common. However, given the prevalence of "consumerist" conceptions of the environment, this concern tended to be related more to the particular preferences of members, consumers and other outside publics than it did to staff, whether they were senior or otherwise. Therefore it was argued that the firm's role was to respond to issues of concern for external publics and to ensure high levels of consumer sovereignty by providing appropriate choice and information.

Nonetheless this was also seen as a moral stance, although different to the personal moral conviction exhibited in the smaller companies. In the CWS, it was seen not simply as a commercial duty, but as an ethical obligation to respond to, and make a stand on, those social and environmental issues which were highlighted as important for consumers; by the same token it would be irresponsible or "nannying" to set their own agenda without an adequate mandate from consumers or members. Thus, CWS's consumer welfare mission was translated into a morally relevant frame of reference for the environment by invoking notions consistent with a societal marketing orientation such as society's and consumers' long term interest. Hence, it was the concept of consumer sovereignty and not the chief executive that was regarded as the principal source of moral rectitude in CWS. 


\subsubsection{Dexterity with Narratives}

The setting of the environment within an explicit moral discourse in the social mission companies (at least at senior levels) unquestionably set them apart from conventional organizations, and this was something that managers were clearly not impervious to. Whilst their status as companies owned either by their members (CWS and the Ecology) or by individuals and/or charities committed to their social missions (BWC and Bioregional) definitely facilitated the incorporation of moral aspirations into the firms' goals, the sense of difference within the firms was broader than strategy alone. Indeed, the overall impression gained by the researcher in interacting with the social mission companies was that they exhibited an informality and openness rarely, if ever, evident in the other more conventional companies in this study. Much freer interaction with staff was allowed, and there seemed little if any attempt to present a unified corporate face. Respondents generally depicted their companies as "friendly" ... "like a family" ... "like a large corner shop" or at least, "better than others I've worked for," especially in the smaller companies ${ }^{52}$.

The companies' social missions were thought by managers to have had a prominent role in establishing this cultural difference with conventional companies, and narratives of "difference" were widely alluded to in these companies. It was commonly assumed by staff that their companies were regarded (particularly externally) as more friendly and more caring than their competitors. One CWS manager described the situation thus:

\footnotetext{
${ }^{52}$ Size was certainly an important issue here. Equally, it should be remembered that at the time of the fieldwork Bioregional only employed voluntary staff in addition to its two directors.
} 
I think people do know that we are different. Its that expectation thing again: people expect the Co-op to be responsible. It has a certain ethos. It has a different ring to it than if Sainsbury's or Tesco's were doing it.

This narrative of difference with other companies, although not always accepted as legitimate ${ }^{53}$, appeared to be invoked or denied according to the exigencies of the situation. Whilst it certainly provided a convincing explanation for the fairly relaxed working environment in evidence, and to some at least represented a source of pride, it was also clear that some executives were concerned that "difference" might suggest to others outside the organization something of a lack of commercial acumen:

For many years the company has suffered prejudice due to our ethics... some retailers, bankers and others in the business world have tended to consider BWC a joke. ${ }^{54}$

Where the respect of industry peers was thought important, managers suggested they had often played down the difference narrative, emphasising the importance of a sober appearance, professional manner, and commercial aptitude in dealing with industry peers. In this way, they would not be disadvantaged either commercially - through being treated as a "soft touch" - or personally - through being accorded insufficient professional respect. Hence, the construct of

\footnotetext{
${ }^{53}$ Differences were much more strongly indicated by senior staff than those on the shop or factory floor.

${ }^{54}$ Source: "Thirty Years of Beauty Without Cruelty Cosmetics: A Profile and Short History of Beauty Without Cruelty PLC", BWC, 1993.
} 
narrative surfing developed in chapter 5 might be rounded out to a more general 'dexterity with narratives' with the addition of the social mission companies. Moreover, where in chapter 5 the intra-organizational attempts at image making by environmental protagonists were considered, we can expand this here to incorporate similar processes at the inter-organizational level.

Clearly then managers in the social mission companies had attempted to mediate the perceived difference with conventional organizations, sometimes emphasising it, sometimes playing it down. Cultural difference was seen as important in terms of constructing identification with the organization, but it was also seen as a potential threat to personal self-esteem and the commercial fortunes of the company. Indeed, the increase in "hard-nosed" and "sharp" business practices, coupled with the hegemony of "marketing" and "commercial" discourses into all forms of organizations were cited as important factors in removing differences between profit maximising and other organizations generally. At Bioregional for example, the transferral of the firm from charitable status to corporate status was seen as relatively unproblematic in cultural terms:

Certainly a lot of the phrases and a lot of the systems are being introduced into the voluntary sector from the commercial sector... so in fact all our work was market-led ... so we've really been very aware of market forces and commercial priorities at the charity.

Hence, managers in the social mission companies were increasingly faced with a tension between the nurturing of their uniqueness and of the need for a business 
culture that matched the sophisticated practices in evidence around them. To some extent, this comes down to a question of which brand values the organizations might seek to develop (Lee, 1997), particularly in relation to the strategic priorities of the firm as considered in section 6.3.

\subsubsection{Disseminating Environmental Values}

This inconsistency towards the institutionalisation of the narrative of difference in the social mission companies was mirrored by a surprising lack of attention accorded to the involvement of employees in the companies' missions. Staff were generally found to have had little or no knowledge of their employers' social missions before joining the companies, even though the firms had frequently drawn labour from local communities. Some senior executives however did suggest that it might be advantageous if employees had "a natural sympathy" with the company's objectives or "some knowledge" of environmental issues, particularly at more senior levels, although this was not stressed as a particularly important issue ${ }^{55}$. Equally, company values did not appear to provide any particular motivation for employees themselves in applying to these firms: the position was principally seen as "just a job", although at more senior levels, social and environmental goals might be "an added bonus" for managers. However, even here traditional economic and career goals remained paramount, as this BWC manager explained:

I liked what it stood for, but it was the job I was going for, not the policies...

\footnotetext{
${ }^{55}$ The only exception to this was at Bioregional where managers claimed that any future employees would have to be environmentally-motivated individuals. However, at the time of fieldwork only voluntary staff were employed at Bioregional.
} 
You've got to work, and so provided its not a company that you disagree with, you do your best job. I was pleased to be offered the job because it was a cosmetics company and because it was not tested on animals ... but if it hadn't of had those policies, I would still have joined.

Equally, there was little indication from respondents that corporate mission or environmentalism had any appreciable affect on their commitment or job satisfaction once they had joined the companies ${ }^{56}$. More importance was attached to the personal integrity of the firm's leaders, since genuine commitment on their part was seen by some employees as source of organizational attachment. Probably most important (at least in the smaller companies) was company size, and the parochial, friendly and inclusive nature of the small social mission companies was clearly instrumental in attracting certain staff, and in subsequently enhancing their satisfaction and commitment. More generally, respondents could be seen to some extent to have been socialized into conventional feelings of pride and commitment to their organizations, and this had been attached to a range of factors, but only to a limited extent to the social goals of the company. Hence there was little evidence that employee commitment was correlated with corporate environmentalism or corporate ethics as suggested by previous theoretical (Hoffman, 1993) and quantitative (Hunt et al., 1989) treatments. These concerns were perhaps considered too abstract compared to more direct correlates such as the integrity of their employers, or the friendliness of the atmosphere.

\footnotetext{
${ }^{56}$ There were some exceptions to this trend in a small minority of cases outside of policy entrepreneurs, mainly at senior levels.
} 
In general then, little commitment from staff had been sought in ethical or environmental terms and this was reflected in a lack of environmental knowledge and concern in relation to their workplace roles. Indeed, even where the company was actively involved in green marketing projects, comments such as "I can't think of anything we do on the environment side" and "there's not an awful lot you can do" were symptomatic of a general feeling of detachment and impotence towards the environment in the lower echelons of the companies. There was however some slight - although far from convincing - evidence to suggest that once incumbent, the social or environmental awareness of the company or its managers might "rub off" on employees. For example, stories were related of how a manager had turned vegetarian since joining one company (BWC), some respondents suggested that the prevalence of green products such as recycled paper and fair trade coffee in the office might influence staff (the Ecology), and a number of managers across the social mission organizations considered that the general climate of re-use and energy efficiency was likely to have had some impact upon staff values. Nonetheless, employees suggested that even should they desire to, they had little time for environmental concern, reflection, nor advocacy of any kind. The pressures of their jobs were such that "practicalities" took precedence over "dreamy" views, particularly in the absence of systems of appraisal and reward which signalled otherwise.

In extreme situations, this might even lead to deliberate sabotage of environmental projects by middle management and rank and file employees due to the additional nuisance or burden imposed upon them. In one example, a group of shop floor workers related how new "green" packing materials had proved so unpopular due to their inferior performance, and their unpleasant 
texture and smell, that their use had been avoided as much as possible, either by mixing with the old non-green alternative or by avoiding them altogether. In another example, the trial of an internal plastic recycling scheme had been aborted due to the unfavourable reaction of shop-floor staff ("it was a pain") and middle management ("it cost too much"). Little attention has been directed in the literature towards the potential for such internal resistance to corporate social programs (see however, Collins and Ganotis, 1973), but its existence here, in organizational climates relatively sympathetic towards moral concerns, is of significance. The prevalence of centralised value systems, and the avoidance of imposing values on employees appear to have distanced, and even disenfranchised employees from the moral dimension of the firms' missions. Without sufficient involvement in, or even communication about these decisions, social and environmental programmes may simply become "the boss's thing", thus creating a sense of futility among employees concerning their ability to affect social policy (Collins and Ganotis, 1973).

Overall then there appeared to be a general impassivity towards the co-alignment of individual and company values in the social mission companies. The focus in the 'deep' green business literature on the development of broad, fundamental cultural change (section 3.4.2) was not reflected in the practices of the social mission companies. The dissemination of values was rarely a deliberate or managed process, and employees were generally left to develop their own interpretations and meanings in environmental terms. There was however at least some limited evidence of a growing recognition that a movement towards cultural integration of environmental values might in some way be desirable. Reasons for this differed between a simple "because its what we're about" in 
Bioregional, to a pragmatic decision in the Ecology to supplement the service offering through employing staff that green customers might more easily communicate and identify with. In CWS, it was generally subsumed within a broader culture change programme intended to develop greater customer focus and marketing orientation. Hence there was some recognition in CWS that staff should understand company values, since the importance of these to customers had been verified by the responsible retailer research. Thus, "getting the company culture down to the people on the bottom" was seen as an important, if difficult, step in establishing the responsible retailing initiative as a credible brand building exercise in a service organization.

Interestingly, in 1995 the CWS-owned Co-operative Bank had already set up such an initiative under the banner of "Cultural Heritage Training". Its avowed intention had been as follows:

This education then enables the participant to understand the links between the original Co-operative Principles and the current Mission Statement and Ethical Policies of The Co-operative Bank ....It is intended to be part of an ongoing form of education that constantly informs all our workforce of the importance of the Bank's brand and the values that our customers have learned to associate with that brand $d^{57}$.

There were some signals then that attention towards the potential benefits of cultural integration was on the increase, especially in terms of attracting more

\footnotetext{
${ }^{57}$ Source: private correspondence with Chris Smith, Group Public Affairs Manager, Cooperative Bank.
} 
environmentally aware employees, and/or socializing existing staff into awareness or understanding of the firms' environmental values. However this falls far short of Welford's (1995) demands for "strong" cultures and shared global environmental values, or of Mintzberg's (1989) conception of a "missionary organization" where the organization's sense of mission is strongly and naturally identified with by members who share intensely its particular ideology.

Mintzberg's (1989) analysis does however provide a framework with which to distinguish between different approaches to the reinforcement of organizational ideology in the social mission companies. For example, attempts in CWS to "get the corporate message across" might be regarded as indoctrination or "evoked identification" (p. 226). By comparison, BWC's attempts to ensure that staff had "some awareness and concern" for animal welfare might be regarded more as "natural" or "selected identification" (p.226) since this can be seen to expand merely the potential for shared beliefs and values. Such issues of culture change are significant then for developing a thorough understanding of the complexity of the greening process; therefore, in order to consider them in more depth, these are returned to in chapter 9 .

\subsection{Conclusion}

The analysis presented here provides some important insights into marketing in social mission companies, and in particular on the role of morality in the process of developing and implementing green marketing practices.

First, it was found that in some cases, environmental protection may be integral 
to the very purpose or mission of business organizations, albeit to varying degrees. Consequently these firms could to said to have practiced a mixture of societal and social marketing. According to managers, firms' strategies for marketing appeared to hinge on two key factors: campaigning priorities and mission positioning. Campaigning priorities relates to the balance between promoting the social cause or promoting the firm's products, i.e. the tendency towards social or conventional marketing. Mission positioning relates to the level of attention accorded to the product augmentation afforded by the firm's mission in attracting customers. Different strategies were evident in different companies, contingent on factors such as product and market characteristics, and the strengths of the company and the priorities of its leadership.

Second, a social mission per se did not mean that green marketing would be more developed than in conventional companies. Whilst an environmental mission was naturally reflected in the development of radical green products, a focus on other social issues could certainly deflect attention from the environment. In general, similar concerns were raised by social mission company managers in relation to green product markets as they were by conventional company managers, most notably: perceived under-performance of green products, and the effects of misleading green claims on promotional tactics. Here however, the consequences were likely to be more significant given the need to develop coherent social and societal marketing strategies.

Third, two different conceptions of societal marketing underpinned green marketing in the social mission companies. One position held that corporate responsibility was needed to develop greener alternatives in the absence of 
consumer demand. The other held that the company's responsibility was to make a stand only of issues of concern to customers. To some extent this reflected differences in social missions.

Fourth, notions of the environment within the company tended to rest with key senior executives who employed discourses reflecting their own roles, responsibilities and interests. Therefore there is little or no evidence from these companies to support Prothero's (1990:95) assertions that "present companies practising societal marketing are possibly doing so from a bottom-up approach".

Fifth, within this top-down management approach to green marketing, executives adopted a number of culture management techniques. This involved constructing the environment as a moral issue - either in terms of the personal belief system of the firms' leaders or in terms of establishing a platform for genuine consumer sovereignty; manipulating narratives of difference with conventional companies; and disseminating environmental values. It was somewhat surprising however to discover that managers paid only limited (though possibly growing) attention to establishing some kind of unity of environmental values in their organizations, and for the most part employees experienced only minor socialization in this respect. Therefore, since their main intention was clearly to influence external publics rather than organization members, the social mission companies might be referred to as "reformers" of outside publics rather than "converters" of organization members (Mintzberg, 1989:231).

Having then discussed both conventional and social mission companies' 
approaches to green marketing, the next chapter will focus on an approach which is different again: business-NGO collaboration. This looks in particular at one example in which conventional companies, social mission companies and an environmental pressure group had all come together in a single alliance to develop specific green marketing practices. 


\section{Business-NGO Collaboration}

\subsection{Introduction}

The previous two chapters focused on green marketing and moral meanings in conventional companies (chapter 5) and social mission companies (chapter 6). Both chapters concentrated on situations where firms were working largely independently from other organizations. This chapter however looks at a very different approach to green marketing: collaboration between businesses (both conventional and social mission) and pressure groups, also known as nongovernmental organizations (NGOs). Collaborations of this kind, or 'green alliances' as they are sometimes known in relation to environmental projects (e.g. Mendleson and Polonsky, 1995; Wasik, 1996; Hartman and Stafford, 1997), were introduced in chapter 4. Basically they represent a situation where one or more business organizations and one or more NGOs both commit resources to a single project in order to achieve a common goal. Findings from one such collaboration are reported here: the WWF 1995 Plus Group (the Plus Group).

The Plus Group is a UK green alliance focused on the forest management of timber sources for wood and wood-based products. Its members are the WWF UK - an environmental NGO - and (in 1996) approximately 75 UK companies involved in the trade of wood, and of wood, paper and pulp products (see appendix B for a full membership list). Company members include both conventional companies, among whom are large retailers such as $\mathrm{B} \& \mathrm{Q}$, Sainsbury's and Tesco, as well as many of their suppliers, (a minority of) 
social mission companies, and also BTC, CWS and Bioregional which have already been the subject of case studies in the previous two chapters. Interviews were conducted with 17 representatives from a sample of 13 member organizations; these individuals were identified by their organizations as their main point(s) of contact with the Plus Group. Whilst the findings presented in this chapter relate mainly to the experiences of the study's interviewees, they should also provide considerable insight generalizable to the total Plus Group, and also, although only a single case study, to green alliances and business-NGO collaboration more generally.

It has been suggested that green alliances such as the Plus Group are a relatively new, but potentially important, strategic approach to green marketing (Mendleson and Polonsky, 1995; Wasik, 1996; Hartman and Stafford, 1997). Through them, companies and NGOs might be regarded as moving from their traditional adversarial relationships towards more constructive and collaborative arrangements (Wasik, 1996; Hartman and Stafford, 1997). However, they have been accorded only limited research attention to date, and few case studies of the scope of the current study have yet to be presented in the scholarly management literature. As such, this research represents an important opportunity to develop theoretical insight into this new and little understood phenomenon; to explore some of the limits of contemporary green marketing; and to analyse moral meaning in a very different organizational context. This last point is particularly important since the involvement of an environmental group in the green marketing process might be regarded as having significant potential to describe a very different moral landscape compared to those considered in the previous two chapters. 
Hartman and Stafford (1997:188) even suggest that a "corporate-wide mindset change" and "building an environmentally-responsible corporate culture" are necessary first steps for corporations in collaborating with environmental groups, and this has obvious parallels with the discussions regarding 'deep' green change in chapter 3.

The structure of the chapter then is as follows: following a brief introduction into the background and workings of the Plus Group in section 7.2, green marketing issues are considered (in rather more detail than in chapters 5 and 6) in section 7.3. Then in section 7.4 , the cultural dynamics underlying these green marketing practices are set out, with attention focusing again mainly on the form and content of their moral meaning. Finally, in section 7.5 , these results will be summarised and some brief conclusions and implications discussed.

\subsection{Background to the Case Study}

The WWF 1995 Plus Group is an on-going alliance formally established in January 1996 (but effectively in operation since $1991^{58}$ ). Its two main aims have been to improve forest management practices, and to develop a market for products utilising an appropriate certification scheme (Bendall and

\footnotetext{
${ }^{58}$ The WWF 1995 Plus Group was established to continue the work of its predecessor, the WWF 1995 Group, which had been formed in December 1991, with a set termination in 1995. Since all of the original 1995 Group members became members of the Plus Group, they shall, for the purpose of this study, be regarded as a single organization. Hence, this work should be regarded as building upon previous research on the 1995 Group, namely Murphy (1996a, 1996b), and Bendall and Sullivan (1996).
} 
Sullivan, 1996). The formation of a green alliance to tackle these problems can be seen as something of a reversal of previous antagonism between environmental groups and business in relation to forest management practices and wood sourcing (Murphy, 1996a; 1996b). Indeed, demonstrations concerning retailers' forest sources - particularly the Friends of the Earth 1991 'Chainsaw Massacre' campaign which saw pickets outside DIY stores brandishing defamatory placards and inflatable chainsaws - were clearly an important catalyst in the creation of the original 1995 Group in the first place (Murphy, 1996b).

In joining the Plus Group, companies have had to pledge themselves to a number of commitments relating to their sourcing of wood-based products, the ultimate aim of which has been to phase out all forest sources which fail to meet standards adjudged to be "well-managed" as defined by the Forest Stewardship Council (FSC) ${ }^{59}$. These are ${ }^{60}$ :

1. Members are committed to the Forest Stewardship Council as the only currently credible independent certification and labelling system with global application.

2. Members are committed to the phasing out of the purchase of wood and wood products which do not come from well-managed forests as verified by

\footnotetext{
59 The FSC is an independent membership NGO made up of representatives from environmental and social groups, the timber trade and the forestry profession. It was formally established in 1993 to evaluate, accredit and monitor certifiers of forest management practice.

${ }^{60}$ These were the criteria at the time of the Plus Group's launch. Source: "Forests Newsletter No.6", issued by WWF-UK, October 1995.
} 
independent certifiers accredited by the Forest Stewardship Council.

3. In order to fulfil point 2 members will aim to be purchasing only certified wood and wood products by 31.12.1999.

4. A named senior manager will have responsibility for implementing the above commitments and target.

5. Progress towards the target will be monitored via six-monthly progress reports. Reports must include a database of wood and wood products used by quantity, type and forest source. Sources must be categorised as (a) certified forest (b) known well-managed forest or (c) unknown and/or not well-managed forest.

6. Members of the WWF 1995 Plus Group may use the Forest Stewardship Council product mark when they are licensed to do so. Other labels describing the quality of management of forest sources will be phased out.

"Well-managed" in this context relates to a range of social, environmental and economic sustainability indicators. The role of the WWF in the Plus Group has been to manage and co-ordinate the project, to assess the companies' progress towards the targets ${ }^{61}$, and to act as a gatekeeper for Group membership. Hence, its contribution to the collaboration has been its environmental expertise and other resources such as administration and marketing expenses. In terms of

\footnotetext{
${ }^{61}$ The WWF's principal sanction for under-performing companies has been de-listing from the Group.
} 
Hartman and Stafford's (1997:189) typology of green alliances then, the Plus Group can be regarded as a "green systems alliance", defined by them as a "collaborative partnership ... to implement economically feasible environmental systems or programmes for the greening of business practices."

In this case then, the environmental system implemented through the green alliance has principally operated through supply chain management, utilizing vendor questionnaires ${ }^{62}$. These are distributed by member companies to their suppliers, and this in turn triggers a series of supplier assessments right back through the supply chain to the forest of origin. Questionnaires are focused on issues of forest source identification and management with a view to encouraging improved environmental management. Companies are committed to making six-monthly reports to the WWF concerning their progress towards their commitments; these are then assessed and the company is awarded a one star (not on track), two star (on track given firm action in specific areas) or three star (on track) rating according to the progress made. Should products be produced with all wood content certified as "well-managed", the firm is able to employ FSC endorsement, namely an eco-label generally known as the "FSC mark" or the "tick tree".

\subsection{Green Marketing}

Green alliances can be regarded as potential solutions to some of the commonly indicated problems of green marketing, especially poor credibility,

\footnotetext{
${ }^{62}$ See Lamming and Hampson (1996) for a discussion of the relative merits of vendor questionnaires and other forms of environmental management through supply chains.
} 
consumer cynicism and consumer confusion (Mendleson and Polonsky, 1995). Although these were certainly important motivations for the formation of this particular collaboration, green systems alliances can be seen to have marketing and management implications beyond communications and promotions policies alone (Hartman and Stafford, 1997). Indeed, the present case study raised, in particular, important issues in relation to marketing strategy, industrial networks and supply chain management, marketing relationships, and organizational buying. Therefore, in addition to the emerging green alliance literature, the findings presented here should be regarded as building upon two important recent studies: Drumwright's (1994) investigation of green organizational buying in the US and Lamming and Hampson's (1996) exploratory work into the greening of supply chain management in the UK.

\subsubsection{Marketing Strategy}

Essentially, the Plus Group was depicted by company respondents as a mechanism enabling the long-term marketing of credible, and environmentally appropriate products. The strategy was long term in the sense that although the alliance itself was intended to be of limited duration (four years each for both the 1995 Group and the Plus Group), the processes and procedures set in place were considered to be on-going:

I don't think it finishes. All that happens is that it will bring these things so far ... and make this a normal part of working practice. As soon as we get to the stage where we are buying from just certified forests then the forestry management will become a monitoring process. 
The project was presented by respondents as providing for credible green marketing because it was seen as a proactive response to the plethora of "meaningless", "unsubstantiated" and "rubbish" claims relating to forest management and sustainability in the marketplace. The independent certification of forest management practices provided by the FSC was therefore described as an effective route for developing a credible eco-label for wood products. However, in this situation, the eco-label only related to a single environmental dimension of the product offering, namely the source of wood, paper and pulp components. Since other issues such as the content of recycled/recyclable material, product processing, product disposal, etc. were not incorporated into the scheme, "environmentally appropriate" is meant here in a strictly limited sense.

\subsubsection{Industrial Networks and Supply Chain Management}

Given the goal of identifying and improving forest sources, the Plus Group alliance clearly centred on issues of supply chain management. However, in a broader sense, the management of environmental issues through collaboration within and around supply chains can be thought of within a context of industrial networks (Cramer and Schot, 1993; Shrivastava 1995a/b). Accordingly, critical importance can be attached not only to the individual firm and how it manages its supply chain, but also to the pattern of relationships and interdependencies of which the firm is part (Hakanssson and Snehota, 1997a/b).

Indeed, the over-riding impression given by respondents of the prevailing networks in which the member firms were operating was of considerable 
scepticism, apathy, or even hostility towards the work of the collaboration. Actors within the surrounding networks (and those in the timber and paper industries were particularly highlighted), were commonly portrayed as determinedly unreceptive to environmental overtures, generally as a result of conservatism, a marked spirit of entrenchment concerning established industry practices (particularly in the face of outside/NGO interference), and a wariness towards what were regarded as the environmental "fads and fashions" in the industry which had not subsequently been translated into increased sales. Effective supply chain management was therefore presented as a demanding, even "tortuous" and "horrendous" task by respondents, particularly given the complexity of the prevailing networks. Individual chains of custody were shown to run to as much as $15-20$ different organizations from the forest of origin to the customer.

Respondents also reported little indication of an overtly positive or proactive response from their networks of suppliers in the face of such increased environmental concern and assessment. Whilst some suppliers were characterised as clearly hostile to the initiative, most common appeared to be an acceptance of, or at least acquiescence to, their customers' wishes and demands:

Working in partnership with suppliers: I think we kind of thought we might be up against a tricky one there ... but so far they've actually been pretty cooperative ... I'm not saying that they are necessarily happy with it, but they are co-operating. 
This generally passive, reserved, and commercially attuned response from suppliers supports Lamming and Hampson's (1996:S58) findings from their exploratory study of supply chain management and organizational greening:

In general, the response of suppliers to initial customer proposals to make improvements in environmental soundness was reported to be cool.... Broadly, suppliers responded to problems only in so far as the customer companies required them to.

Evidently more striking to some managers however was the apparent ignorance on the part of their suppliers concerning the source and type of the timber involved in their products, particularly as this constituted the "meat and drink" of their business:

The thing is a lot of them are not aware how vulnerable they are. Its ignorance. You ask them where their wood comes from, what species it is, and they can't tell you. Its been a surprise to me really how much they don't know about their business. Any manufacturer should know where his raw material comes from and how much he gets from there.

Nonetheless a number of respondents who had been involved in the alliance for some time also gave some indication that supplier responses improved and quickened over time as a consequence of their increasing familiarity with the issue, and a refinement of questionnaire formats by their customers. This might be regarded as indicative of the emergence of stronger marketing relationships being developed within the collaboration. 


\subsubsection{Marketing Relationships}

An important contribution of the networks perspective on business-NGO collaboration is its consideration of inter-firm relationships, and also the placing of these relationships within wider industrial interactions. The notion of relationships was acknowledged by respondents with respect to both immediate marketing relationships with their suppliers and customers, as well as strategic relationships with other Plus Group constituents. Indeed most interviewees spoke of "working with" and "consulting with" their alliance partners and with their suppliers to achieve the aims of the Plus Group. For example the co-ordinating role of an independent NGO in the initiative appeared to assist in enabling companies to interact with their competitors on issues of mutual concern:

We went out for the first time to Sweden and Finland. I was consulting with other members of the Group ... getting together and saying: OK I'm going out there this week, what stage are we at with the process?

In this way, the potential communication and information flow problems associated with managing environmental issues through supply chains, such as those highlighted by Lamming and Hampson (1996), could be overcome. Also, long-term and consistent supplier relationships were seen as an easier context than 'spot market' trading in which to trace the chain of custody for timber. Managers however put even stronger emphasis on the value of "partnership sourcing" in lubricating the channelling of environmentally certified products through the supply chain. This necessitated the "nurturing" and "supporting" of supply relationships to encourage vendors to adopt 
greener sources themselves. The question of maintaining existing supply relationships or switching to newer "greener" suppliers however was a critical one within this context (Lamming and Hampson, 1996). In general, the former rather than the latter seemed to be preferred, probably due to a mixture of inertia and uncertainty-reduction (Ford, 1980), power-seeking (Frances and Garney, 1996), and/or managers' interpretations of corporate social responsibility within their companies (Drumwright, 1994). One supplier for example described this mixture of rationales underlying retailers' purchasing strategies thus:

Basically you can't get them to change their buying philosophy. They ... said to me something like: we have a very strong ethical buying policy. And I said: Oh really?. Yeah, he said, so you wont be able to persuade the buyer to buy your product just because its environmental! It was extraordinary!

A few respondents (including the one above) did however offer a few clear examples of supply relationships evidently based on buyer support and sustenance. More frequently though this discourse of "partnership" marketing relationships conflicted with corresponding accounts of the need for "buying clout" on the part of member firms in their purchasing role in order to "pressurise", or at least "encourage" suppliers into acquiescence. A respondent from one of the large retailers in the Plus Group described the situation thus:

Simply [the suppliers'] business acumen tells them they should often beg, bow and scrape to [respondent's company]. But you know the kind of customersupplier relationship that often exists - where the customer has such a large 
value to the supplier that they'll do anything they can to please them? Not anything, but within reason. And that is the way it works.

It was clear then that much of the espoused desire for partnership sourcing tended to be either wishful thinking on the part of respondents, or a rhetorical device that masked less equitable supplier relationships based on compulsion and persuasion from (in particular) the large retailers (Frances and Garnsey, 1996). The length, frequency, and response rate for questionnaires also appeared to reflect resource dependence and the purchasing power of the buyer relative to the supplier (Pfeffer and Salancik, 1978). Hence those members less able to mobilise buying power clearly settled for shorter questionnaires, slower turnaround times, and more chasing up for questionnaire responses. Resource dependencies of this type were also compounded by the concentration of Group membership in particular industrial networks, thus effectively locking in a sizeable majority of individual firms' potential suppliers/customers.

At the level of individual marketing relationships, executives in this study tended to suggest that the most effective way to encourage those further back in the value chain to embrace the alliance's certification route was through the invocation of commercial arguments rather than moral ones. Indeed, executives from subordinate (in power terms) suppliers of member firms indicated that there were immediate commercial paybacks from joining their customers in the Group, making them, as one respondent put it, "instantly one of their best suppliers." Equally there was some recognition that subordinate suppliers expected to be played off against each other on environmental criteria just as they would be on economic criteria. For example, an executive 
from one supplier suggested that the gaining of FSC accreditation by one company would be "used as a stick to beat the other suppliers with". Accordingly, it could be argued that some suppliers felt under pressure to switch to certified products from customers (and potential customers) who they did not always believe were fully committed to the process and who did not necessarily offer corresponding support.

\subsubsection{Buying Centre}

The nature of industrial networks, supply chains and the marketing relationships within them were clearly of importance in tracing the marketing dynamics of the Plus Group collaboration. Moreover, it was apparent that the relative importance of the ethical or environmental dimension within these relationships was dependant upon where within the member companies these relationships were initiated and maintained. Certainly the lack of procurement executives as company representatives in the Plus Group, and the lack of involvement of buyers generally in the alliance, might be regarded as a significant problem, given that the project was centred on organizational buying and supply chain management (Murphy, 1996a) ${ }^{63}$. Hence, the role of company respondents as environmental protagonists within their companies frequently allowed them only to initiate and influence the buying decision process rather than make the final purchase decision (Drumwright, 1994). Indeed, although some of the company representatives had been instrumental in their company approaching the WWF, for many of them it was simply a job

\footnotetext{
${ }^{63}$ In the Plus Group as a whole, out of 73 executives identified as the main representatives for their companies in the Group, only 6 were dedicated procurement staff.
} 
which had been assigned to them, and was concerned primarily with having an input into existing supply relationships rather than initiating new ones. Equally, a number of Plus Group representatives were not themselves responsible for sending out and chasing up supplier questionnaires, only the collation, interpretation and presentation of responses. Their main role in the buying process then was that of attempting to inform, advise and encourage the final decision makers along the path of timber certification.

\subsubsection{Green Marketing and Social Responsibility}

Whilst the alliance itself might be regarded as an environmentally responsible project along certain dimensions, respondents suggested that the organizations within it had various rationales for joining, some more rooted in notions of corporate social responsibility than others. Indeed, different orientations towards social responsibility within organizations can impact significantly on green purchasing strategies and tactics (Drumwright, 1994). Hence, in order to distinguish between different approaches, such marketing activities can be usefully categorised in terms of their economic, non-economic, or mixed objectives (Drumwright, 1996).

\section{Economic objectives}

Several respondents expressed a clear conviction that their organization's involvement in the Plus Group was purely on account of economic objectives associated with adding value, maintaining or improving sales, and/or building corporate/brand image. Whilst a few respondents even suggested that all companies could be subsumed within this category, purely economic 
objectives were particularly evident for those members of the collaboration either totally or principally located within industrial marketing networks, and who had joined in response to the "recommendation" or "pressure" applied to them by their customers (principally retailers) who were already members of the Plus Group. Therefore, for many of the industrial marketing companies in the alliance, membership was presented as a strategy aimed at securing distribution channels for company products, driven in the main by a desire to guarantee and retain existing customers through current channels rather than attracting new business and/or developing new channels.

\section{Non-economic objectives}

A few respondents (and not only those from the WWF and social mission companies) were emphatic in their conviction that their organization had joined as a result of social purpose or moral conviction, usually on the part of senior management. The issue had usually already been a major concern for the company, and the alliance simply represented an obvious tactical route forward. In some cases (for example Bioregional as considered in chapter 6) the company had even emerged partly as a result of the formation of the alliance. Whilst these claims for purely social objectives could potentially be interpreted as a disguise for other more commercial motives, respondents themselves at least appeared entirely convinced, as the following two examples illustrate:

When [the respondent's manager] asked us to look into it we actually asked to see him on his own for a bit and said: Look, why do you want to do this? And it was when he said, well he wanted to be doing the right thing for his children 
and his children's children, and really that is what it comes down to, that we felt comfortable with what he was asking us to do. At least we knew that it was being done for the right reasons, not for some kind of PR scoop.

We are doing it because we want to. There's no real advantage to us. For instance, the logo: the only time one of our customers would see the logo would be if they were committing suicide, because then they're face-to-face with the [product].

Although for these companies, non-economic objectives could be seen to have driven the project, this does not mean that economic benefits were necessarily absent altogether only that when they were in evidence they were perceived to be merely "spin-offs". Nonetheless, of those respondents citing their companies within this category, none suggested further that they had adopted what might be regarded as the extreme ethical position, namely that they deliberately would not take advantage of such "marketing spin-offs".

\section{Mixed objectives}

Probably the largest group of respondents cited mixed objectives for joining the Plus Group, stressing the alignment of economic and non-economic goals similar to the enlightened self-interest position adopted by the conventional companies in chapter 5 . This was particularly true of the consumer products companies, especially the retailers in the alliance, given their direct contact with the public. Interestingly however, respondents from these companies tended to under-emphasise direct marketing advantages and positive product augmentation, and preferred instead to articulate marketing dimensions in 
terms of avoiding commercial disadvantages either through price increases, quality shortfalls, or reputation problems. Hence, companies had joined "because we wouldn't be at a commercial disadvantage by doing it", rather than for marketing advantages: "well yes, I guess there are some [advantages] ... but the general public probably don't know anything about it, but then that's not the prime reason we are doing it". Respondents emphasised in particular their refusal to accept cost increases from suppliers or price increases on their own products as a result of joining the alliance. Indeed, one of the principal attractions of the initiative for many organizations appeared to be the possibility of passing on, or at least sharing, the costs of appraisal, verification and certification with other firms in the supply networks centred around the source forests.

The principal reasons forwarded for the relatively peripheral concern with marketing benefits for the mixed objective companies were in line with those discussed in chapters 5 and 6, namely consumer scepticism for green claims generally; the relatively small number of highly concerned green consumers; and the continual dominance of price and performance product evaluation criteria. Accordingly, many respondents indicated that source certification would only provide a significant positive augmentation if all other product criteria were equal with respect to competing offerings. Hence, it was considered unlikely that environmental certification would have a substantial impact on sales or market share ${ }^{64}$.

\footnotetext{
${ }^{64}$ It is important to note that at the time of data collection, few certified products were yet on the market, and managers were generally very cautious in their estimations of the potential appeal to consumers.
} 
Interestingly, some respondents from other alliance members (particularly those specifying their objectives as non-economic) tended to depict some of the large retailers as rather more concerned about positive marketing benefits than perhaps did the retailers themselves:

I get a bit fed up when I'm at the WWF seminars listening to the supermarket squabble... they are trying to score points over each other for a start; and also they seem to want to almost hijack the process. They're in it for the commercial side of things primarily because they believe that they'll get an advantage to their company by being able to say well our products come from certified sources. OK fine, but [respondent's company] doesn't really have that motivation behind it.

Nonetheless, long term or "strategic" objectives, particularly those centred around the building and consolidation of corporate reputation, and maintaining "industry best-practice" were however cited as important, although this appeared to be driven more out of a desire to be "squeaky clean" in environmental terms (i.e. avoiding negative augmentation) than it was a desire to publicly extol their own virtues (again a 'muted greening' strategy). Hence, it was the mixed objectives evident in the avoidance of potentially bad publicity (such as that encountered by the DIY sheds in advance of the formation of the original 1995 Group $^{65}$ ), and the moral obligation to back up marketing claims which had motivated their entry into the Plus Group:

\footnotetext{
${ }^{65}$ See Murphy (1996) for a description of the development of the Group and the role of pressure group action in prompting action from the DIY chains.
} 
At the time, [competitors] were being targeted by Friends of the Earth, with great blow up chain-saws outside their stores saying you know, 'Destroying Tropical Rainforests!' which was having ... an impact on their business. So that's why they joined. We joined because it wasn't being highlighted as an issue for us but we were uncomfortable with making those claims.

\subsection{Cultural Dynamics of Green Marketing}

In general then, respondents from alliance members suggested that social responsibility might be a factor in their involvement in the project, although this not only varied in its extent, but also in the meaning and significance applied to it by the organizations and individuals involved. Whilst the previous section sought to provide some description of the different organizational contexts in evidence within the collaboration, it is more useful to explore these different interpretations by looking at the cultural dynamics underlying the operationalization of the project within these contexts. Analysis of executives' responses revealed some common themes which appeared to provide significant explanatory power in determining how moral meanings were constructed within the alliance. Broadly speaking these can be categorised as sub-cultural divisions, cultural mediators, managing symbolism and moral circumscription.

\subsubsection{Sub-Cultural Divisions}

The specific network of relationships created by the Plus Group, in combination with the nature of the buying centres in member companies, could be seen to have precipitated significant sub-cultural divisions within the 
domain of the alliance. Most significantly, respondents indicated substantial cultural difference between themselves (who as their organization's representatives in the alliance might be regarded as environmental policy entrepreneurs) and those vested with the responsibility of putting the policy into practice at a day-to-day level, i.e. company procurement managers and staff. For example, many of those company representatives interviewed were specialist environmental staff, and these individuals tended (not unnaturally) to stress the importance of their having substantial environmental knowledge and expertise, and also the ability to employ sophisticated environmental management techniques in order to expedite the project within member organizations. Even non-specialist representatives tended at least to express some interest or concern for the environment, presenting themselves, as one respondent put it, as "enthusiastic amateurs".

In contrast to the environmental policy entrepreneurs, buyers were predominantly described by interviewees as "hard-nosed" business people, driven mainly by concerns for margins and costs rather than for the ethical or environmental dimensions of goods. This was evidenced in statements of the kind: "all they are interested in is screwing down the current supplier another $5 \% ", . .$. "they're not environmentally conditioned", etc. In many ways this was similar to Drumwright's (1994:13) findings in the US, where purchasing executives were shown "to ignore opportunities for socially responsible buying and resist the initiatives of others". However, in this particular case it was especially pertinent given that it was the area of procurement that provided the essential mechanism through which the collaboration could operate and succeed, i.e. the purchase of wood products by organizations. 
The sub-cultural divisions evident in the alliance also extended beyond the member companies and into the WWF itself. In particular, the project team responsible for the running of the Plus Group was presented as culturally distinct from other sections of the organization, especially with respect to their commercially attuned frame of reference for environmental knowledge:

[A WWF project manager] is ... extremely credible, obviously the sort of person that can interact well with business. He's an ideal person for the job ... realising you can effect change by working with some of the forward thinking industry. And how deeply that culture goes beyond [the Plus Group project team], I don't know. But I think its quite good you've still got people like [a WWF fieldworker] with a huge big bushy beard lost in the rainforest because that's the other side of what the organization is doing. And I don't think [the WWF] would have complete credibility without having that other side.

Clearly within these sub-cultures, individuals might in many respects display a healthy diversity. However, substantial unity was certainly evident in terms of the cultural knowledge of these groups, particularly since individual roles, goals and systems of assessment tended to reflect these sub-cultural divisions. Perhaps the best example of this was provided by respondents' descriptions of the differing understandings of notions of competition and co-operation within the two main corporate subcultures (buyers and policy entrepreneurs), labelled here the 'co-operation-competition paradox'.

\section{Co-operation-Competition Paradox}

Whilst a network perspective on industrial marketing and purchasing 
highlights substantial inter-relationships and exchange contingencies among firms not directly connected through dyadic relations (Anderson et al., 1994), a broad alliance such as the Plus Group can be seen to situate companies in the particular scenario of working with some of their main competitors as well as against them, even in highly competitive sectors such as grocery and DIY retailing. Indeed, a few of the early alliance members had clearly been at least partially instrumental in their competitors joining the Plus Group early on in its development, even to the point of "recruiting" some of these other companies. Moreover, many respondents expressed a certain satisfaction, even humour, at working with their alliance counterparts in companies which might more usually be regarded as fierce competitors:

It's comical actually because I phone up my competitors ... and say, "I want to speak to so and so, it's [respondent name] here from [respondent's company]." And you hear them go: "What?! What are you doing?" Ha ha. They say: "Are you sure? Or are you taking the mickey?" ... so I've got to know them personally; and when I joined they said if there's any questions you've got, get in touch.

Whilst at the time that the fieldwork was conducted many respondents indicated that the size of the collaboration's membership was sufficient for the project to succeed, member companies were still presented with something of an anomaly in that the collaboration was likely to operate more successfully (by having greater "clout") the more of a company's competitors that joined the project, but at the same time this was likely to erode any marketing advantages that membership might bring in terms of creating a differential 
advantage against these same companies. As one respondent put it:

From a business perspective .... anything that gives me competitive advantage is going to be welcome .... so from that perspective I'd be happy if everybody stays away and gives us a free run. On a broader scale, ultimately it's got to become better if more people become involved and committed to it.

Consequently, policy entrepreneurs were faced with competing preferences with regards to encouraging alliance membership. Even more significantly however, there was a paradox in how such individuals defined their companies' relationship with other members of the collaboration. They were at the same time both collaborators and competitors:

"Our competitors .... are competitors in terms of selling [products], but they are allies in terms of reducing environmental impacts".

In one sense this apparent paradox may have generated conflicting "network identities" among the firms concerned (Anderson, et al., 1994). Whilst alliance representatives appeared to have recognised this, the co-operation-competition paradox was successfully rationalized in terms of the sub-cultural divisions within their organizations and how these appeared to have diffused throughout the network. In particular, alliance policy entrepreneurs could be seen as sharing environmental orientations and a common environmental goal; equally they were not personally in direct competition with each other. In contrast, those personnel seen as most in competition with each other, the company buyers, were rarely involved directly in the machinations of the Plus Group. 
Hence respondents could stress their companies' co-operation on environmental issues (what the policy entrepreneurs themselves did) within a context of market competition (what the buyers did):

Us and our competitors .... come together. You haven't got buyers there, you've just got environment people, so its a lot less competitive atmosphere, more co-operative .... When the Group members go over to visit the forests, they all go over together because our aims are common. You wouldn't get a bunch of buyers visiting a supplier together because they're in competition together.

In short, network organizations were not seen by respondents as monolithic entities, but as micro-collections of cultural groups, each of which had its own role to play, and with its own network bonds. Hence, the success of those actors directly bonded by the alliance (in this case company representatives), might be said to be strongly dependant on those not bonded by the alliance (here, the corporate buyers), since it was these that had control over linking critical network resources (timber-based products) through marketing exchanges (Hakansson and Snehota, 1997).

\subsubsection{Cultural Mediators}

The existence of such sub-cultural divisions can be seen to have had important ramifications for the cultural dynamics of the alliance, and in particular for the moral framing of the environment within this context. Most notably, certain individuals and cultural groups within the alliance were thrust into the role of cultural mediators, i.e. they were required to bridge gaps in cultural 
knowledge between disparate groups within the alliance. Hence, their role was to act as depositories of specific environmental knowledge, interpreters of environmental meanings, and architects of appropriate, and ideally shared, frames of reference.

The Plus Group project managers in the WWF were cultural mediators in that they had to translate the relatively radical environmental concerns of their organization - sustainability, biodiversity conservation etc. - into a business issue with business objectives, business opportunities etc. Their success in this respect was demonstrated by the generally positive views of corporate respondents' with respect to the aims and structure of the project:

They've been able to set some practical goals. They've set up some tangible steps in that they have this specification standard for getting certification for the forests and there are people that will do the certification, they will do the check. So they will deliver to us effectively somebody that is as sound as you can get in terms of the source forest.

Not all company respondents however were as sanguine. Although generally considered supportive and approachable, several individuals depicted the WWF as sorely lacking business acumen and "too short-sighted businesswise" to be able to "appreciate commercial pressures". Indeed, these perceived shortcomings were further considered to potentially threaten the smooth running of the collaboration:

In terms of how that works with the companies, you are not dealing with a 
competent strategic alliance partner, the WWF: they are under-resourced, they've been running at a standstill and they are not as clear thinking as they purport to be, in my view. Things keep changing ... and it is quite difficult.

Perhaps surprisingly, those respondents least convinced of the WWF's commercial credentials and most aggressive in their castigation of the organization's lack of commercial-mindedness tended to be (although not exclusively) environment managers and external environmental consultants. It might be assumed that these particular individuals would be more likely to have had especially pronounced environmental orientations, and would therefore be more in support of the WWF rather than less. It is probable then that this dynamic resulted from some kind of role competition. Hence, the companies' environmental experts may have either consciously or unconsciously seen the WWF as competing with them in the role of cultural mediation, resulting in a subsequent playing down of their NGO partner's credentials in understanding the position of the commercial partners.

Indeed, company policy entrepreneurs could be seen to be themselves cultural mediators between what they regarded as the commercially reasonable, but still relatively "idealistic" demands of the WWF and the "commercial priorities" of their companies. In this way they could be regarded as helping both sides to see the position of the other in what might otherwise be a relationship between incompatibles:

Pressure groups are in the business of campaigning and they are not in the business of making other people's lives easy. I'm not in the business of being 
good to pressure groups. And the businesses are selling what they sell .... so its a constant tension between everybody's needs. And we try to smooth the waters - I'm not sure successfully, but we try.

Most respondents seemed to attest then to a process of mutual learning, facilitated it seems by this series of cultural mediators whose role emerged from the particular organization and relationships which typified the Group. These mediators - be they WWF employees, external consultants, or company employees - re-interpreted and re-framed the environmental problems at the heart of the alliance in order to involve and motivate those who were more commercially-oriented and in more competitive positions than themselves. These dynamics are mapped in figure 7.2.

Whilst figure 7.2 should not be taken as a definitive map of the dynamics of the Plus Group, it should throw some light on the steps in the communication and mediation processes within business-NGO collaboration. Essentially, individuals within the alliance can be thought of as occupying positions on twin scales of business-environment orientation and co-operation-competition orientation $^{66}$. Accordingly, both competition and business orientations can be said to gain in strength as communication proceeds from the WWF to the buyers that implement the Plus Group policy for their companies in the marketplace. The role of cultural mediators (indicated by the shaded regions) is to facilitate the smooth communication and meaning transferral between

\footnotetext{
${ }^{66}$ Obviously, not all individuals in the same role will share identical orientations, nor can those orientation be given quantitative values; equally, the diagram makes no attempt to depict the entire scope of the Plus Group, nor all the communications therein.
} 


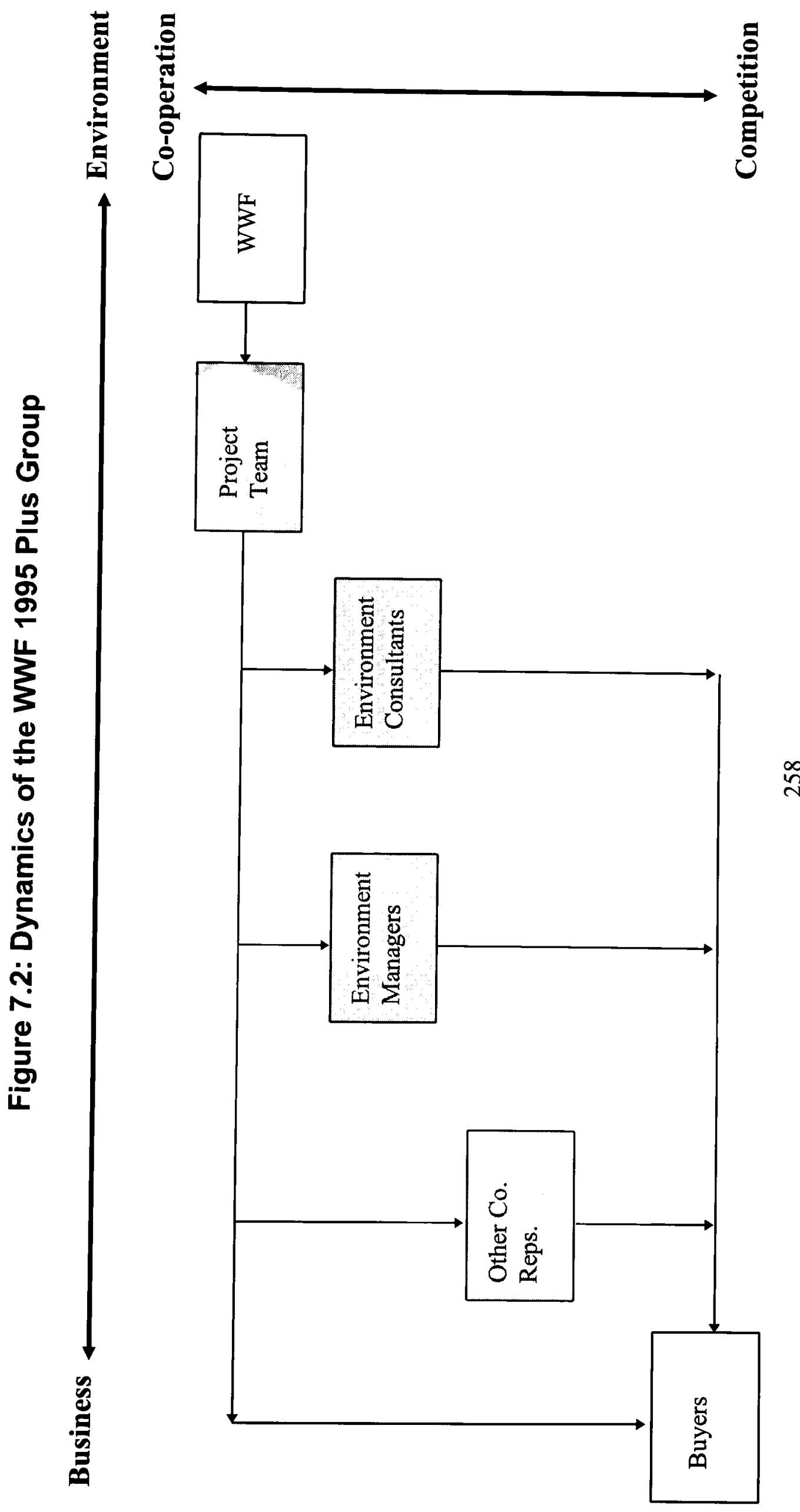


these culturally disparate groups within the alliance.

\subsubsection{Constructing the Environment}

It was apparent that this process of mediation had significant implications for the construction of the environment within the domain of the alliance. Most notably, there was strong evidence to suggest that the effect of serial reinterpretation of the group's purpose, objectives, and importance was to successively remove layers of moral meaning from the notion of forest conservation. The moral imperative so clear in the conservation mission of the WWF thus tended to be stripped away in order to make the project more meaningful for factions within the alliance with more advanced commercial and competition orientations. For example, buyers were perceived by the more environmentally-oriented representatives as needing commercial arguments with clear directives and performance incentives, and not moral arguments, in order to act upon the policy on a day-today level:

How I go about [motivating the buyers] - all I can do is try and make the case ... show how it relates to customers and how ... there's a danger of losing business ultimately if we don 't actually come up with ... the [certified product] that those people are going to want to buy.

This process of re-interpretation and re-framing was abetted by range of cultural and symbolic activities across the member organizations. Given the varying orientations and goals of collaboration members, those keen to ensure its success sought to render the image and associations of its members as compatible, or at least acceptable, and this necessitated a substantial degree of 
manipulation and management of various symbols (Mendleson and Polonsky, 1995).

\section{Managing Symbolism}

A number of elements within the alliance appeared to be managed at the symbolic level, and these could be seen to have profound implications for the moral meanings established and communicated within the Group. First, the particular environmental subject of the alliance had to be seen to have wide appeal to various alliance constituencies. Certainly, the issue of forest certification had clear commercial resonance, and the symbolic potency of forests, trees, and hardwoods as dangerously "emotive" and "sensitive" public relations issues was widely acknowledged by executives in this study. Moreover there was some recognition that the erosion of forest reserves was clearly detrimental to commercial expansion and, given that the narrow focus of the project prevented any need to reflect at length on wider (and more challenging) issues of reducing timber consumption and increasing recycling, the issue was relatively easily translated from a moralistic, "emotive", environmental issue into a compelling, "rational", business argument.

Second, respondents emphasised the symbolic importance of top management endorsement and support for the alliance. In this respect, the task of mobilising support for the initiative in member organizations was expedited by the obligation within the alliance to secure board level patronage. Thus, whilst the direct and tangible involvement of senior managers was rare in the larger 
organizations $^{67}$, the signature of board members on internal memos, or particularly on correspondence to suppliers, provided the project with more significance and weight for otherwise recalcitrant factions within the domain of the alliance:

When we sent out the questionnaires, it wasn't coming from me or from [the other Plus Group representative], it was coming from ... the boss. We ... wrote the letter for him to sign, so you've got these [suppliers] getting something from the top man - they can 't ignore it. They'd be very foolish if they did.

Third, the importance of constructing a correct and unchallenging image for the project meant that an appropriate vocabulary had to be established and accepted. Therefore terminology for appropriate forest management was steered away from the semantically suspect "sustainability" towards the more agreeable term "well-managed" (Murphy, 1996b). Discussions with respondents were indeed littered with references to the term "well-managed" and to other even less environmentally explicit terms such as "managed" and "certified" whereas the use of "sustainability" terms was rare ${ }^{68}$. In addition, company respondents tended to stress the issue of maintaining forest resources (with its obvious commercial resonance) to the virtual exclusion of other FSC

\footnotetext{
${ }^{67}$ Drumwright (1994) also attests to the low profile of top management in successful green purchasing initiatives. This conflicts with much of the existing prescriptive green business literature (e.g. Elkington and Burke, 1989; Welford and Gouldson, 1993).

${ }^{68}$ Unlike the commercial partners, there was not a complete avoidance of "sustainability" terms on the part of their NGO partner, neither by WWF respondents themselves, nor in communications to other audiences. It is also important to note that the FSC had utilized "well-managed" in defining its principles of forest management.
} 
principles such as conservation and diversity of forest fauna and indigenous people's rights. Executives in mediator roles then had clearly adopted a vocabulary with little obvious moral or environmental symbolism and which exhibited little semantic baggage which might be considered inappropriate or challenging to business interests. Whilst this may have been seen as important in terms of managing the image of the project in order to lubricate the challenging process of meaning transferral and re-interpretation, it may also give some indication that fundamental environmental values were either being suppressed or exorcised by these individuals and groups.

Finally, the role of cultural mediator also involved a considerable amount of personal image making, similar to that described for the conventional companies in chapter 5 (section 5.4.3). For example, attempts by individuals in the WWF to create an overall impression that was as acceptable to business as possible prompted an approach much like the "professional management" approach of the policy entrepreneurs in BTC. As one WWF respondent put it:

There is an attempt to be extremely professional. I wasn't formally a shorthaired, suit wearing person. I was a long-haired forest ecologist who knew all about trees.

Also, extra staffing needs were satisfied by bringing in an external consultant who was apparently selected as much for their business credentials as their environmental skills. The professional image and commercial approach taken on by the WWF as a consequence did not go unacknowledged by the commercial partners: 
[The consultant] ... has a commercial background. They are aware that they can't afford to have a stereotyped environmentalist approach. They can't be woolly and indeterminate on things. They can't have high moral principles and ideas but not have actually any degree of a sense of reality with it because there's no way they'll get to tie business in.

Essentially then, for the most part the adoption of a cultural mediator role was seen as important for the success of the collaboration. However, the acts of symbol manipulation and image management associated with this role tended to successively strip emotional and moral meaning from the issue of forest sustainability in order for it to be an attractive and workable proposition for other network members, and in particular for the groups and individuals that were actually required to implement the project on a day to day level in commercial organizations. Clearly, commercial or enterprise discourses were privileged above moral discourses in the collaboration. Despite this, there was at least some evidence of moral meanings remaining attached to the environment, and to forest conservation in particular, although this was highly circumscribed into particular moral arenas, as shall be shown next.

\subsubsection{Moral Circumscription}

Whilst certainly not the only possible route for coupling the environment with morality, some respondents suggested that the alliance itself, and formal meetings in particular, could act as an important forum that allowed (or in some cases perhaps compelled) individuals to consider the environment as a moral issue, and as an end in itself rather than as simply instrumental to business goals of long-term survival and profit. Congregations of 
representatives from those organizations involved in the collaboration were as one earlier respondent described it, characterised by a "less competitive atmosphere, more co-operative". This was seen by some as a result of a congruence of values and deep moral convictions concerning the environment amongst many of those involved in the project. As a result, this environmentally supportive context could act as a catalyst for the re-surfacing of side-lined or forgotten personal values:

When they join the group and they have someone to talk to who has got a likemind (or it might be a like-mind like they were when they joined the company 15 years ago) then it can get their enthusiasm going again. But it can work both ways as well.... There's people I know in companies that are really serious, totally serious, totally committed, as committed as I am about what we are doing. And it is enormously heartening. I love it from that point of view.

Therefore, moral meanings were not completed eroded in the acts of constant re-interpretation by the cultural mediators since a favourable climate could resurrect, or create, an enthusiasm for greening which might otherwise be submerged under the socialization processes associated with more conventional cultural norms and behaviours in firms. Nonetheless, a distinctly moral conception of the environment was relatively rare in the Plus Group collaboration, and to some extent appeared to be circumscribed into particular limited arenas where it might arouse less hostility or suspicion. Therefore, although the discourse of the environment in this case was almost completely amoralized in the need to establish a common and acceptable frame of 
reference within business organizations (as was also the case in chapter 5), this did not mean that moral meanings could not be translated in certain contexts. It would appear however that this moral circumscription was fairly limiting and generally became more so as communication moved away from the NGO partner and towards more morally antagonistic factions such as the procurement function (right to left in figure 7.2).

Given the potential scale and scope of green alliances and supply chain management approaches, such forums may also have impact beyond their borders, such as through educating consumers (Mendleson and Polonsky, 1995) and suppliers (Lamming and Hampson, 1996). In the present case, many thousands of questionnaires were (and continue to be) distributed throughout wide-ranging industrial networks, reaching people and organizations where before there may have been little knowledge or interest, let alone concern for forest management. This might be regarded as providing greater potential for the activation of personal reflection on the ethics of corporate policies and practices:

There ... have been several hundred thousand questionnaires gone out all over the world. So that wherever you go, wherever you meet a forest manager, they've probably had - or somebody they sell to has had - a 95 Plus Group questionnaire. And it really makes people sit up and take notice when it's somebody they've got a commercial relationship with asking them about their environmental performance.

Moreover, this re-interpretation and circumscription of morality in relation to 
green marketing activities may also have implications for how such understandings are communicated to consumers, although with so few certified products on the market at the time that fieldwork was conducted it was too early to ascertain this empirically. Nonetheless, since one of the requirements for inclusion into the alliance was the removal of all claims relating to forest management from firms' non-certified products, it could be said that the presence of an NGO collaborator in companies' green marketing practices at least has the effect of censuring unethical claims, and correspondingly adding credibility to 'ethical' or appropriate ones. Hence the circumscription of moral meaning may potentially be extended to include consumers and other publics external to the alliance.

\subsection{Conclusion}

This chapter provides considerable insight into the practice of green marketing through business-NGO collaboration, and also to some degree through supply chain management. Moreover, analysis of the particular moral landscape that such an approach was found to describe contributes significantly to an understanding of the way in which notions of morality are handled and transferred in settings characterised by such cultural heterogeneity.

Green alliances are based on an assumption of mutual dependence amongst businesses and pressure groups in relation to the environment. The evidence of the Plus Group case study however is that this dependence can also be extended to incorporate wider network interdependencies between firms and their chains of suppliers/customers. Hence, it is reasonable to suggest that alliances widen the scope and domain of green marketing, bringing it into 
broader strategic and industrial policy debates. Critically, the importance of environmental criteria in industrial marketing exchanges appears to be, for the most part, contingent on the nature of existing relationships, characterised primarily by issues of loyalty, power differentials, and resource dependencies. Hence, the environment remains a subordinate issue in industrial marketing networks (or at least those centred on the timber and paper trade), highlighting the important role for rigidities in industry attitudes in dampening green marketing projects, irrespective of the concerns of individual firms or managers.

Despite being termed here a green alliance, the case of the Plus Group illustrates the range of motivations behind individual firms choosing to collaborate with pressure groups, some more based around social and environmental responsibility than others. For many organizations there was found to be an over-riding economic rationale for collaborating, for a few there was a convincing moral justification, and for the largest number, a combination of the two appeared to provide the necessary impetus. Again, as in chapter 5 , the avoidance of potential negative product augmentation and other marketing disadvantages rather than an explicit search for competitive advantage through positive augmentation appeared to drive firms towards environmental initiatives.

Moral meanings were also relatively understated in the dynamics of the alliance at an operational level. Its domain embraced substantial cultural diversity both within and between organizations. Broadly speaking this diversity could be categorised in terms of sub-cultural divisions representing 
differing personal and group environmental beliefs and orientations. It was found that the need to bridge these divisions resulted in certain individuals and/or groups being thrust into the role of cultural mediators such as to translate appropriate environmental and moral frames of reference between them. The cultural mediator can be thought of as a complimentary role to that played by the policy entrepreneur as shown in chapters 5 and 6 , and in some instances, they appear to be analogous.

As a result of the manoeuvres of the cultural mediators, moral meanings were successively stripped from conceptions of the environment such that it could be presented in an appropriate guise for acceptance by important factions within the alliance network. This was found to be particularly due to the strategies employed by the cultural mediators, many of which were similar in form and execution to those revealed as part of the policy entrepreneur's weaponry in chapter 5 . Most notably, the management and manipulation of symbolism was found to be central to the phenomenon in this case.

In the final analysis however, the amoralization of the environment in the case of the Plus Group was not as complete as in BTC in chapter 5, but considerably greater tham in the social mission companies in chapter 6 . First, it was stripped away through successive re-interpretation, suggesting that some (probably decreasing) elements of moral meaning remained attached to concepts of the environment as they were traded throughout the alliance network. And second, distinctly moral discourses clearly prevailed in certain circumscribed arenas, suggesting parallels with the social mission companies, given their strong concentration of moral meaning in the upper echelons of the 
organization.

Whist a few comparisons have been made, it has been the intention in these last three chapters to set out each of the three organizational approaches to green marketing largely in isolation from the others. The rationale for this has been to allow for the full richness of analysis of each case to come out, thus taking full advantage of the exploratory research approach. Attempts to standardise the reporting of results into set categories and constructs at an earlier stage may have stymied the emergence of some of the more important insights and findings revealed here. Having explored these different approaches in some depth then, it is now possible to draw some of these findings together, to locate them into existing scholarly literature, and to suggest what implications might be drawn from these results. This is the aim of the next two chapters. 


\section{Green Marketing and Morality - Evidence from Three Approaches}

\subsection{Introduction}

This study is an analysis of whether, and in which ways, morality is given or denied meaning and expression in green marketing practices. The previous three chapters have provided descriptive accounts of the green marketing process and its cultural dynamics in three organizational contexts conventional, social mission, and NGO-collaboration respectively; various elements pertaining to the moral dimension of these processes and dynamics have been set out, leading to some preliminary construction of theoretical categories, constructs and propositions regarding morality in marketing. In these next two chapters the results presented in chapters $5-7$ will be brought together in a more coherent manner than has been attempted thus far, and will be summarised, compared, explained, and their implications examined.

This subsequent discussion is organized around four key themes, two of which shall be considered in this chapter, and two in the next. These are: green marketing in practice, and morality in green marketing (this chapter); and moralization and amoralization in green marketing (the next chapter). These four themes capture the main findings provided by the study with respect to the basic research questions set out in chapter 3 (section 3.5) as well as providing a means to develop further on these findings and locate them successfully within existing organizational and management literature. 
This chapter will proceed then with a discussion of contemporary green marketing practice as observed in the case organizations. This covers both strategic and tactical (marketing mix) issues, before going on to set out a more grounded theory of green marketing. This is followed by an examination of where and how morality was found to have impacted upon green marketing. Here, the classification of moral arenas in marketing which was introduced in chapter 2 is utilised (i.e. macro and micro moral domains), and implications are drawn for both the 'deep' and the 'shallow' conceptions of greening which were introduced in chapter 3. Finally, the main points in the chapter are summarised and some brief conclusions set out.

\subsection{Green Marketing in Practice}

Environmental considerations had impacted upon marketing, in one form or another, in all of the sampled organizations. For some, products with environmental attributes were being developed, or were currently marketed; in others, environmental considerations were less directly product-related, and impacted mainly on packaging and distribution systems, or communications policies. This is not to say that many firms laid claims themselves to be conducting green marketing; and the fact that some even vigorously denied it might be construed as evidence that the possible internal contradictions of the term - as highlighted by the critical 'deep' green position (see section 3.4.2) were acknowledged and felt keenly by respondents. As for definitions of green products, there was little consistency across the sample. However, for most respondents the concept of a green product was generally taken to imply either explicit green brands, or products with green attributes of some kind. Nonetheless, it was recognised that the grounds in this respect were constantly 
shifting. What were once green attributes (e.g. zero-CFC propellants or recycled packaging), and therefore worthy of green claims, had in many instances become industry norms, sometimes even legal requirements (National Consumer Council, 1996).

In general, environmentally branded product offerings were seen by most respondents as having achieved only limited success in the 1990s marketplace. Many respondents indicated a disparity between survey evidence reporting strong environmental concern on the part of large proportions of end consumers and the limited pro-environmental purchase behaviour effected in the marketplace. As such, evidence of the greening of consumers was either viewed with suspicion, or else seen more as a indicator of a potential future demand for green products. Wong et al. (1996) report similar findings, suggesting that the principal reasons for disappointing performance of green products can be located in both supply-side issues and consumer demand preferences.

In the case of supply-side issues, respondents in the current study laid much of the blame on miscreant firms who had confused consumers with inappropriate and misleading labelling, thus confounding any straightforward marketing approaches (Prothero et al., 1994; Kangun and Polonsky, 1995; National Consumer Council, 1996). Kangun and Polonsky (1995) for example have identified three main reasons behind consumer confusion with green claims: a) the difficulty in assessing environmental claims, even after purchase; b) the high context-dependency of many claimed benefits; and c) consumer unfamiliarity with environmental claims, terms and (relatively uncertain) scientific evidence. 
Moreover, respondents also indicated problems that had arisen from maladroit attempts by competing firms to introduce green products which had clearly failed to convince consumers of their efficacy. As Wong et al. (1996:269) also found, "potential consumers' perception of green product performance was often cited as a major barrier to consumption of the product." Over and over in the current study, respondents at all levels across the range of sampled organizations repeated the same cautionary tales of doomed green product introductions by niche firms which had failed to match the perceived performance of conventional offerings. These "salutary" products were seen as having achieved environmental benefit only by sacrificing the immediate appeals and satisfactions sought by consumers (Kotler, 1972). In particular, the case of green detergents and washing-up liquids appeared to be an important story in shaping organizational perceptions of consumer preferences for conventional performance standards over environmental benefits. This story, in its various tellings, crossed organizational as well as industrial contexts. This was one manager's version:

They produced product that was inferior and hoped to sell it to the fanatical fringe... If you look at the washing powders that came out - the so-called green powders - I don't think there is one left on the market. Because they did not perform in the way that the non-environmental powders did... People thought they could get away with it; the public's expectations were considered to be less for an environmental product.

This story was widely used to justify a number of assertions, in particular: consumer dislike of green brands; performance problems of green products; 
and "cowboy" green marketing from competitors. At the same time however, some respondents confirmed that these apparent green trade-offs were frequently more perceived than real, since they were based on inaccurate perceptions on the part of consumers (Peattie, 1995). For example, the manager above later commented:

Its like washing powder, or detergents, to be fair. Detergents do not produce soap suds; the manufacturers of detergents add a sudser so that when you open the door, you see frothy soap bubbles on the top. It doesn't help clean the clothes - but it makes you think its washing clothes properly... But what is more environmentally friendly? Detergent without froth in. We spend a fortune in treatment plants taking the froth out that we put in just to satisfy the customer!

Essentially then the green detergents story can be regarded as a widely accepted myth which helped to create and sustain meaning about the marketing of green products. Myths can be regarded as "unjustified beliefs, often enshrined in stories, and which influence how organizational actors understand and react to their social situation" (Brown, 1995a:15). In this instance, the detergents myth was used to help in explaining and creating cause and effect relationships under conditions of incomplete knowledge, regardless of whether it was factually true or not (Boje et al., 1982). In addition, on the occasions that the internal contradictions of the myth were acknowledged by respondents, this was generally to further develop the prevailing picture of consumers as not only confused, but even irrational in the context of green product offerings. So how were firms to respond in the face of such confusion, 
suspicion and irrationality on the part of consumers?

\subsubsection{Strategic Responses}

Fineman and Clarke's (1996) study of industry responses to green stakeholders reveals that consumers generally have less impact on organizations' environmental responses than do campaigners, regulators, and internal champions. The evidence in the current study was generally supportive of this finding, with green marketing programmes apparently being more substantially motivated by a desire to minimise the threat or impact of environmental legislation or pressure group attention (especially in conventional or collaborative organizations) or to reflect the ideals of the leader/powerful factions (especially in social mission companies) than to take advantage of explicit market opportunities.

Hence, with purchasers of both consumer and industrial products generally seen as unwilling to experiment with explicit green products, and vendors rarely enthusiastic about providing substantial marketing support, the muted greening strategy widely attested to by executives in this study quite logically focused attention on those issues highlighted by powerful stakeholders rather than seeking to uncover the minutiae of consumer's green preferences. In this sense, models of green marketing strategy and marketing such as those presented by Peattie and Ratnayaka (1992) and Simintiras et al. (1997) are correct in their attention to describing the range of green driving forces, although perhaps overly emphatic in the role accorded to green consumers in pushing forward the issue. However, green strategy in practice can be seen to be more complex than the off-the-peg typologies presented by Peattie and 
Ratnayaka (1992) among others. Clearly, green marketing strategies are changing, evolving and emerging as firms experiment and learn about the complexities of various issues, and about the role and reactions of various stakeholders. Therefore, organizations can be seen to operate more than just a single strategy, but rather experiment with a number of approaches as they attempt to adapt to the shifting business environment.

Overall then, it was evident from this study that the key strategic decisions of green marketing were not so much locked within the marketing department itself, but within interactions with other constituencies, particularly those in other organizational functions, those in the product supply chain, and those powerful stakeholders external to the organization. In the case of the former, the perceived strategic importance of the environment was found to be highly dependant on the championing of certain policy entrepreneurs across organizational divisions, particularly in the absence of unequivocal support from the market (Dillon and Fischer, 1992; Drumwright, 1994; Fineman, 1996, 1997; Fineman and Clarke, 1996). Such internal selling of green initiatives might only rarely be initiated from those in marketing functions, often rendering the task highly formidable, unless activated from the executive suite or other powerful centres in the organization. Menon and Menon (1997) regard these intra-functional efforts as indicative of an attempt to move from a "tactical" to a "quasi-strategic" mode of green marketing. Equally, given their attempts at creating "frame braking" change in the industry, the social mission companies might be regarded as adopting a "strategic" orientation under this schema, particularly since green initiatives tended to emerge from more senior levels in their case. 
A second issue of importance regarding organizational interactions is that of value chain constituents. Since environmental value and/or costs are likely to be associated with various different stages in the value chain, consideration of the full environmental impact of any given product needs to extend beyond the boundaries of the individual firm (Peattie, 1992). Hence, environmental management practices such as cradle-to-grave stewardship and lifecycle analysis inevitably involve the individual firm in taking account of upstream and downstream activities in the supply chain (Lamming and Hampson, 1996). This could be seen as suggesting to many firms that supply chain alliances might be an important strategic option (Cramer and Schot, 1993; Shrivastava $1995 \mathrm{a} / \mathrm{b})$. Whilst radical greening initiatives appeared to be impeded by a mixture of apathy, inertia, uncertainty-reduction and conservatism within the product supply chain, the incremental development of environmental products by existing supply chains incumbents was argued by respondents to provide the stability for long term and gradual environmental improvements (Cramer and Schot, 1993; Lamming and Hampson, 1996). The adoption of an alliance strategy to address such issues might then be a reasonably efficient and effective manner of generating leverage through the supply chain, although this is clearly reliant upon existing vendor-vendee power differentials, and also the level of influence of environmental protagonists upon the industrial exchange process (Drumwright, 1994).

A number of collaboration respondents suggested also that "partnership" between competitors on green product issues could be important from a strategic point of view. This could provide the means to acquire environmental expertise and knowledge as well as consolidating horizontal channel power in 
order to influence supply chains more effectively (Varadarajan and Rajaratnam, 1986; Hamel et al., 1989; Jarillo and Stevenson, 1991; Bucklin and Sengupta, 1993); moreover, competitor collaboration could also help in establishing a stronger voice with which to present defensible environmental positions and arguments to other stakeholders (Schot, 1992; Simmons and Wynne, 1993). Effective response to powerful green stakeholders could also be seen as an important factor in driving firms to collaborate with NGOs, as in the Plus Group initiative. This could similarly provide access to environmental expertise (Bendall and Sullivan, 1996; Hartman and Stafford, 1997) and in addition, perhaps more importantly, help to co-opt what might otherwise be sharp and very public critics of the firm (Fineman and Clarke, 1996). Indeed, there appeared to be some consensus amongst the sample of corporate respondents that working with powerful stakeholders - especially regulators and pressure groups - in a proactive manner might be preferable to a responsive strategic mode since it allowed for some possibility of shaping the business context (Sethi, 1979).

\subsubsection{Marketing Mix Policies}

\section{Product}

The recent history of limited market success for green products in all but relatively narrow niches had clearly impacted significantly upon the current green marketing tactics of sampled firms observed in this study. Disappointing market performance was widely cited by respondents as evidence to illustrate the inadvisability either of developing more fundamental product modifications, or of foisting radical green brands into the market. Hence the 
most preferred product strategy for the conventional firms was what Schot (1992) refers to as a "re-use strategy", i.e. a concentration on identifying uses and markets for re-used and recycled product. Environmental protagonists in BTC referred to this as "closing the loop", although it could be seen to be far more atomistic than Shrivastava's (1995a) model of an industrial eco-system, described in chapter 3 (section 3.4.2).

According to Schot (1992) the introduction of re-use product strategies in current practice is focused on protecting existing products and market shares more than it is on maximising the use of natural resources. This is because it avoids any need for the development of fundamental product and/or process innovations. Indeed, radical product introductions were generally eschewed in most cases, with notable exceptions being in the small social mission companies. Most common overall then was a focus on gradually improving the environmental performance of existing products, such that various discrete elements of individual products were incrementally greened over time rather than going through any kind of fundamental environmental overhaul or transformation. A number of respondents likened this to the commitment to continuous improvement implicit in the development of TQM. Therefore, although environmental considerations were found to be entering the product development process, they were "seldom a factor in determining whether a product is made or which product is made," (Schot and Fischer, 1993:11).

This pattern was not repeated however amongst the three small social mission companies where senior executives claimed that the firm's social mission had to be mirrored by their products. Indeed, overall the green product attributes 
offered by the social mission companies were in many respects the most radical, not because it was what customers demanded, but because it was what the leaders wanted to produce. According to most marketing textbooks, this might be thought of as representing a selling concept approach as opposed to an approach embracing the marketing concept or even or the societal marketing concept (see e.g. Kotler et al., 1996). Only two out of the four social mission companies (Ecology and Bioregional) were observed to offer product attributes which took account of a far greater number of environmental impacts than was considered normal for the industry. Hence, the inclusion of certain social or environmental attributes into a firm's product offering should not be assumed to indicate a general predisposition towards incorporating a greater range of environmental features into products. Prothero and McDonagh (1992) for example have found that firms offering cosmetics on a cruelty-free platform are not necessarily concerned with other environmental attributes, and might even lag behind conventional firms in researching new green product developments. Moreover, the evidence from the current study is that the assessment and measurement of social and environmental impacts were often more formalised, and in some respects better developed and more sophisticated in the conventional and collaborative organizations than in the social mission companies.

Packaging could be seen to have been a prime initial target for greening, both in terms of reducing product packaging and re-using or recycling transport packaging ${ }^{69}$. In the majority of cases this had brought with it cost savings as

\footnotetext{
${ }^{69}$ This refers only to conventional and social mission companies - packaging was not directly included in the Plus Group initiative.
} 
well as environmental benefits, representing clear examples of win-win solutions. The larger companies such as BTC and CWS had focused mainly on limiting the negative impact of impending legislation, particularly in the context of the targets for packaging reductions, recycling and recovery set by the 1994 EC Packaging Waste Directive. As one manager commented: "We started with packaging. One, because of the Packaging Directive; and two because it was very easy target to make gains on." The prospects of the introduction of a landfill tax (eventually introduced in October 1996) which set levies according to the amount of waste going to landfill ${ }^{70}$ had crystallised the importance of the issue to managers. And with new packaging regulations imminent, these firms had recognised the importance of developing more sophisticated assessment systems for "auditing" packaging usage, as well as logistics systems to manage its flow more effectively. To this end, respondents spoke of the importance of creating long-term contracts with trade recyclers in order to facilitate the flow of packaging and packaging waste, and to buffer the firm from rapid fluctuations in prices for its recyclable materials. In this way, the threat of regulation could be turned into a steady, if small, income. Thus, even where recycling and re-use initiatives had been originally motivated by environmental concerns, they were quickly crystallised into economic costs and benefits. For example, the following account describes one firm's decision not to introduce recycling of plastic transportation packaging:

We trialed plastic recycling for 2 weeks in (unit A). We were very careful and diligent in making sure that all the right plastic went in there .... and in one

\footnotetext{
${ }^{70}$ At the time of writing this levy was $£ 7$ per tonne, except for specific "inactive" wastes where the charge was $£ 2$ per tonne. These levels are expected to increase over time.
} 
week's turnover of say 11000 , all we got was $1 \mathrm{lb}$ or $1 / 2 \mathrm{~kg}$ of plastic. Multiply that by the total turnover, say $£ 4 m$ - that's 2 tonnes of plastic! $£ 14$ of landfill levy! And that's after all the efforts by staff, say $£ 1000$ in personnel costs, plus all the problems of getting balers and getting the plastic back from the (units). Its just not worth it.

In the smaller companies, packaging was simply "minimised" and "re-used where possible", rather than systematically managed. Regulation was, if anything, perceived as a positive step forward since it curbed some of the excesses of their larger competitors. Where they experienced most problems though was in failing to attract trade recycling companies to collect packaging due to their lack of scale.

\section{Price}

The lack of success for green products identified by respondents was also attributed in part to a general antipathy on the part of consumers towards paying premium prices for them. Only relatively small segments of the market were seen as prepared to pay for green attributes - a phenomenon mirrored, and in some cases amplified further, in the case of industrial products. Nonetheless, this did not appear to have been seen by many firms as a signal that such marketing mixes were inappropriate, but rather that consumers wanted "something for nothing". Accordingly, the use of aggressive penetration pricing to build initial demand had been eschewed, leaving new green brands without a viable pricing policy (Wong et al., 1996). This in turn further eroded the possibility of green brand introductions being perceived as a viable strategy for sizeable market segments. 


\section{Promotion}

In terms of promotional tactics, only those firms with an explicit environmental mission continued to favour an approach which called considerable attention to environmental features, and even this was observed to vary according to the prominence of the firm's mission in its positioning strategy. In general then, strong green claims were avoided. Indeed, there was considerable evidence to suggest that executives even regarded these as potentially harmful additions to marketing effort given consumer preconceptions of performance short-falls (National Consumer Council, 1996; Wong et al., 1996), problems of poor credibility (Kangun and Polonsky, 1995), and the prospect of increased scrutiny from pressure groups as a result of public declarations of greenness (Fineman, 1996).

More common among the total sample was a 'muted' approach whereby environmental attributes were to some extent played down, either by reducing the promotional budget of green products (Wong et al., 1996), or by relegating green claims to on-pack labelling claims or to infrequent, propitious public relations and publicity opportunities. At times, green claims were only implied through the design of product packaging. These findings correspond with those from the National Consumer Council's (1996) extensive study of green claims on consumer goods which claimed to show a shift since 1991 from green advertising to on-pack claims and logos. However, the National Consumer Council (1996:1) was sharply critical of these claims, suggesting that many of them were: "unverifiable, and/or vague, woolly, specious or misleading. Most are accompanied by a bewildering range of logos and symbols." 
One of the main problems of on-pack green claims is that they have been largely unsusceptible either to current legislation or to industry codes of practice (National Consumer Council, 1996). Some moves to increase regulation within the EU however were in evidence at the time of fieldwork, particularly in the area of claims regarding animal testing (Brown, 1996). Over the range of sampled companies, such moves to increase regulation were only really welcomed by the social mission companies, who saw it not only as a good thing in itself, but also as a potential prop to their otherwise ailing "ethical" competitive advantage:

I'm with (the EU labelling directive). I support it. Animal labelling has been abused beyond recognition .... this could mean other companies will have to consider the replacement of meaningless slogans with factual statements ... which we've being doing all along of course.

In addition to legislation (and as a means to forestall it), growth in businessNGO alliances during the 1990s is indicative of a parallel movement away from self-endorsement towards external certification and validation of green claims (Mendleson and Polonsky, 1995; Bendall and Sullivan, 1996; Hartman and Stafford, 1997). The Plus Group alliance, as one such initiative, was certainly seen by most of its members as a positive and significant step towards redressing consumer confusion and cynicism regarding green claims. Nonetheless, for those companies dealing in a wide range of products outside the remit of the alliance, it remained an experimental approach in one product area rather than one which was to be introduced across all ranges. As such it did not appear that the "bewildering" number of claims and logos identified by 
the National Consumer Council (1996) would actually be reduced in any substantial way, even if the number of unsubstantiated ones might. For the consumer then, a general perplexity towards green claims in general seems only likely to be diminished as a result of further legislative amendments (National Consumer Council, 1996).

Green communications can also be seen to be part of a wider move by businesses to restore and/or consolidate legitimacy in the face of increased public environmental concern (Patten, 1992; Tombs, 1993). The need to convert other constituencies to the environmental worldview of the organization appeared to be a significant force in driving various communications efforts for the firms in this study. This was most explicit in the case of the social mission companies where the relative commitment to campaigning was seen as a critical distinguishing feature of their marketing strategies. However, all of the sampled organizations were observed to have communicated their environmental position at some point to some external (and to a lesser extent, internal) audiences, usually in the form of public relations and publicity campaigns. In the main, such communication appeared to be aimed at legitimation of the form and scope of environmental management at the organization, focusing primarily on information provision and persuasion of the "truth", "responsibility" or "rationality" of the organization's approach (Tombs, 1993). For example, an executive at BTC explained the development of environmental material for schools thus:

You've got to get right back to educating - or getting involved in the environmental education of children - at an early age to try and help them 
understand and get rid of some of these fallacies that are around with regard to environmental issues. So that if environmentalists are coming through, and coming into our stores and deciding whether to buy our products, they do it on the basis of some of the facts, rather than what they might read that's more emotive sort of journalism.

This construction of discourses of rationality and irrationality, "facts" and "fallacies" clearly has the purpose of excluding critical constituencies, and might be regarded as an attempt to deny the possibility of critical voices (Puxty, 1986; Tombs, 1993). A more progressive strategy however might involve engaging in more open, participatory and trust-based dialogue with various audiences (Tombs, 1993; Prothero et al., 1994; Peattie, 1995; McDonagh, 1995). Indeed, there is some evidence from the findings reported here, as well as elsewhere (see Schot, 1992; Simmons and Wynne, 1993), that some conventional and social mission companies might, for various reasons, be increasingly willing to engage in dialogue with stakeholder groups. However the extent to which this is actually open and participatory in practice remains to be seen.

\section{Distribution}

Finally, the role of distribution has been shown to play a critical role in the green marketing process, despite remaining relatively under-researched to date (Simintiras et al., 1997). In one case (Bioregional), distribution systems were at the heart of the environmental strategy; this however was not only not valued by potential resellers, but valued negatively by them. Consequently, competitive advantage had to be sought elsewhere. Indeed, the willingness or 
otherwise of channel members to accept green products, or even the principle of greening in itself, has been shown to be a key concern for many firms (Wong et al., 1996). For the social mission firms in this study, retailer reluctance to switch from favoured suppliers to greener new entrants was experienced as probably the major stumbling block for marketing success and for the diffusion of green innovations more generally. In other cases of course, the opposite was true: green marketing initiatives of re-sellers (such as the Plus Group initiative) had driven pro-environmental change on the part of suppliers.

Fineman's (1996) in-depth account of the reaction to environmental issues in the UK grocery multiples reveals the importance of different cultural styles in determining the particular response of retailers. His picture is one of conservative adaptation to green concerns in retail organizations where managers have been well socialized into feelings of commitment to the well drummed culture of social responsibility, and clear hostility in those less so. Here, it was found that although the relative approbation of the prevailing culture of industrial purchasers was an important determinant of the success of green product distribution, the role of environmental protagonists in the buying decision was also critical (Drumwright, 1994). The mediation effected by such individuals between sub-cultural interests within (and around) the firm, and their relative influence within these constituencies, was found to have significant impacts upon channel behaviour. 


\subsubsection{Green Marketing Theory}

Having then examined the practice of green marketing by looking at the evidence from three different approaches, is it possible to set out a theory, grounded in this qualitative data, which can describe or explain this phenomenon satisfactorily? Given the balance of the proceeding discussion, it is evident that from a theoretical perspective, the practice of green marketing is perhaps most appropriately conceptualised in terms of a network of internal and external interactions. This assertion however stands in sharp contradiction to most of the existing green marketing literature which tends to focus on the atomised organization (denying external interactions), and treating it as a monolithic entity (denying internal interactions). However, not only does an interactionist model appear to best reflect the reality of green marketing practice as observed in this study, but it also accommodates well the deep green perspective on organizations which highlights issues such as systems structure, interdependence and reciprocity (see Stead and Stead, 1992; Shrivastava, 1994, 1995a/b). Hence, it is argued here that the best theoretical perspectives on green marketing are likely to be those such as the networks model of marketing exchanges and stakeholder theory, both of which accommodate such interactions fairly successfully.

The central proposition of the networks model is that firms' marketing exchanges can best be studied within the context of the broad pattern of interrelationships of which the individual firm is but a single element (Easton, 1992; Hakansson and Snehota, 1997a/b). This model however has been accorded only limited attention in the green marketing literature to date, reflecting perhaps both the continued focus of marketing theory on individual transactions rather 
than relationships (Gummesson, 1992), and the fact that the theoretical foundations of the networks model have little or nothing to say about the role of the environment in these relationships (McDonagh, 1996). It does however help to conceptualise green marketing issues as pertaining not simply to individual firms, but to whole systems of inter-related exchanges. Environmental impacts are not simply the result of individual exchanges, but of the behaviour of input and output chains (Cramer and Schot, 1993; Peattie, 1995; Shrivastava, 1995a; Lamming and Hampson, 1996), and of competitors (Schot, 1992; Peattie, 1995; Shrivastava, 1995b), among others. Indeed, executives in this study repeatedly stressed that the intensity of green marketing policies was dependant on the extent of supplier and customer support, "competitive pressures" and whether or not there was "a level playing field" in the marketplace.

There are relationships however which are critical to green marketing and which the networks model does not appear to accommodate very satisfactorily. These are those within the organization (especially those including parties not integral to the exchange itself) and those with organizations involved in noneconomic exchanges and other relationships with the organization (e.g. pressure groups, regulators, the media etc.). In this respect the stakeholder theory of the organization is a more appropriate model with which to conceptualise green marketing. Although, the stakeholder model has been most commonly associated with social responsibility and social performance generally, there is a growing body of literature associated specifically with organizational greening (e.g. Stead and Stead, 1992; Fineman and Clarke, 1996). However, the utilisation of this theoretical framework to green marketing in particular is still noticeably underdeveloped beyond some 
consideration of the pressure for greening associated with these groups, and exhortations to involve (at least some of) them in the green marketing policy process (see Charter, 1992; Peattie and Charter, 1994; Mendleson and Polonsky, 1995; Wasik, 1996).

Perhaps the greatest strength of the stakeholder model though is that it not only allows for consideration of formal and informal relationships between constituencies internal and external to the organization, but can also take account of the relative power, influence and expectations of these constituencies on the behaviour of the organization and its managers (Donaldson and Preston, 1995; Fineman and Clarke, 1996; Johnson and Scholes, 1997). This offers considerable conceptual space for green marketing theory since, as it has been shown, green marketing practice can largely be explained in terms of the influence of internal and external stakeholders, and the ability of the former to mobilise and mediate the latter to further particular goals. Hence, it can be reasonably argued that stakeholder theory provides a good descriptive model of a range of green marketing behaviours ${ }^{71}$. It is however outside the scope of this thesis to attempt a more complex theory of green marketing outside of this relatively uncontroversial assertion. Given that the focus here is on the moral dimension of green marketing, it is sufficient for our ends to leave that particular discussion there, and to move on to the central

\footnotetext{
${ }^{71}$ As it was reported in chapter 1, Donaldson and Preston (1995) argue that there are two other conceptions of stakeholder theory in addition to its descriptive form, i.e. as a normative model of how organizations should behave, and as an instrumental model of how organizations can improve their performance. Whilst it is not the intention to explore these dimensions here, they certainly demand further attention in the future in order to develop a more complete theory of green marketing.
} 
concern of morality.

\subsection{Green Marketing and Morality}

In chapter 2, two principal domains of morality in marketing were introduced and discussed, namely macro and micro questions. These shall be returned now in order to summarise and assess the moral dimension of green marketing as observed in the sampled organizations. Particular attention is paid to providing insight based around the 'shallow' vs 'deep' perspectives on green marketing as discussed in chapter 3 . It should be remembered that this study has taken an analytical perspective on morality in green marketing, centred around the notions that organizations can be studied as cultures, and that morality might be examined as a cultural phenomenon. Therefore, it is timely to re-iterate here that this has entailed some bracketing of the researcher's own normative frame of reference in order that the conceptions and interpretations of respondents themselves might be foregrounded. Hence, it has been attempted as much as possible to allow respondents to apply notions of morality to green marketing as they saw fit, rather than introducing the researcher's own categories, definitions, or judgements.

\subsubsection{Green Marketing as a Moral Subject}

In the main, questions regarding the morality of green marketing as a practice in itself was approached through establishing how individuals and organizations integrated green marketing programmes within concepts of social responsibility, i.e. to what extent was the provision of environmental benefits through marketing activities presented as a moral duty or role for the 
organization?

Substantial variations were observed in this respect. First, respondents in a number of organizations (particularly managers in some of the Plus Group alliance members and Flygt, as well as a number of shop floor employees) saw the marketing of green products as divorced entirely from notions of social responsibility. Here, the environment was not presented in terms of being a social concern for the business or a moral dilemma to contend with, but merely as another element in the daily routine of supplying timber or sewage treatment technologies, or whatever (Jackall, 1988). Hence, only if customers demanded environmental attributes, and they could be delivered at a greater profit than would be forthcoming otherwise, would they be supplied. Protestations of ethically-driven greening (e.g. by colleagues or other organizations) were often seen by these respondents as attempts to hoodwink a gullible public, and "to jump onto a bandwagon" of environmental concern. This might be regarded as consistent with the Friedman (1988) position, namely that it is only through pure self-interest that the firm satisfies its social responsibilities of profit maximisation; questions of ethics beyond the standard "rules of the game" are thus merely diversions, and therefore unethical in themselves.

The Friedman argument however does not capture the whole breadth of this type of response. Articulating a different ideology, but ending up with curiously similar conclusions, a minority of these respondents (all from shop floor positions) came close to a deep green perspective. This argument centred on the claim that environmental problems were either not properly, or not 
realistically, within the remit of businesses and of the market, and therefore should be handled at a governmental or even at a personal level. These selfavowed "sceptics" or "pessimists" might be generally equated with those 'deep' green politics writers introduced in chapter 3 (section 3.4.2) who saw green marketing as essentially an oxymoron. The fact that they were of low hierarchical status might be seen as indicative that managers in contrast might progress through some degree of socialization process whereby role and organizational attachments begin to impact upon their green moralities (Fineman, 1996).

In the case of BTC, and a large proportion of those companies in the Plus Group alliance, enlightened self-interest positions were articulated, such that it was argued that environmental benefits should only be pursued when associated with economic benefits. Central to this position however was the contention that the financial interests of the firm and its social responsibilities were unlikely to be in conflict (Abratt and Sacks, 1989). Whilst in general this centred on denying that costs were likely to increase through "responsible" greening programmes, there were also suggestions that pragmatic responses to green stakeholders would be sure to benefit the firm in the long run through brand building, reputation enhancement, stakeholder satisfaction, etc. However, this ignored any recognition that the costs of environmental programmes might spiral once the limited number of cost-reducing, win-win solutions were exhausted (Walley and Whitehead, 1994), as well as green arguments that current costing procedures fail to internalise true environmental costs (Bebbington and Gray, 1992; Gray et al., 1993). As it was argued in chapters 5 and 7 , this very upbeat and positive view of the commercial benefits 
of greening was closely associated with the need to legitimate environmental projects in the eyes of otherwise reluctant and suspicious organizational constituencies.

Respondents from a number of other (particularly social mission) organizations were more comfortable with ascribing the introduction of green marketing programmes to ethical motives of social responsibility. These were probably closest to the visions of 'deep' green marketing presented in chapter, although in most cases a strong 'shallow' element prevailed. In the main, this distinctly moralized green marketing corresponded with strong signals from the top of the organization which indicated that the adoption of a firm voluntary stand on the environment was defensible without concomitant commercial justification (Fineman, 1996). Indeed, to some extent, for these respondents the vagaries of customer demand were seen as an unstable platform on which to build green strategies compared to a moral impulse from within, particularly given the distortions in the market brought on by the recent history of misleading green claims.

We recognise that environment is a very low priority for most people ... and most consumers will opt for the cheapest, or what gives them the right combination of quality and price, and won't take much notice of environmental criteria ... although there is a lot of scope for corporate responsibility in that [retailers] put out on the shelves what they want to sell ... in many cases its just as a company that's what we stand for - to do things in a certain way, to have a regard for environmental principles. And I'm sure we lose orders on that basis. 
Organization size, bureaucratisation, and professionalisation appeared to be important factors here, for these brought with them the need to couch greening in more formal, dispassionate discourses rather than in personal, emotional and informal terms (Jackall, 1988; Fineman, 1996; 1997). Hence in CWS, the largest social mission company considered here, increasing professionalisation as a retail organization had meant that social responsibility was increasingly presented in terms of a brand value rather than as a moral principle. For example, environmental initiatives would be justified on grounds of positioning rather than ethics:

Through the provision of customer bring-to recycling points and internal recycling schemes, the CWS's positioning as the 'Responsible Retailer' is enhanced in the eyes of the consumer ${ }^{72}$.

Therefore, managers emphasised how resources for social objectives should principally be deployed according to stakeholder demands - and in particular consumer tastes - much as resources for economic objectives were, rather than according to any moral precept. Respondents in the other companies claiming social responsibility meanwhile continued to explain this in terms of the personal moralities of their leaders, and not a responsiveness to customers and other stakeholders.

If, as it was argued in chapter 3, the main point of departure for the radical critiques of green marketing is a rejection of the pursuit of ever-expanding consumption, it is clear that only very few of these examples where green

\footnotetext{
${ }^{72}$ Source: CWS Retail Waste Management Group: Management Briefing, March 1996.
} 
marketing was presented as a moral subject (perhaps only Bioregional and the Ecology) corresponded with a true 'deep ecology' position. Even the valuable effort to explore sustainable options represented by the Plus Group still had a long way to go before it could be considered as a means to sustainable growth. Its focus on a single product attribute (wood sources) meant that there was no attempt to consider the sustainability of end products as a whole since this would also have to include other components, production methods, and distribution systems, etc. Hence, we might regard these other more common positions as distinctly 'shallow' in their conception.

\section{Societal Marketing Concept}

It was stated at the end of section 2.2.3 that one of the intentions of this study was to attempt to flesh out the notion of the societal marketing concept (SMC). Although some discussion was presented in chapter 6, it is appropriate to widen the discussion here to explore the extent to which any of the approaches analysed had embraced the SMC. Certainly of the sampled firms, only perhaps the small social mission firms Bioregional and the Ecology (and to a lesser extent BWC) could be in a position to argue that all of the goods and services provided by them had taken into consideration their overall social impacts, and the long-run welfare of consumers and other citizens. This was clearly due to the personal convictions or conscience of their leaders, informed as they were by previous (often continued) engagements with environmental groups and organizations. Certainly these were not simple marketing orientations since the managers involved freely asserted that these positions would be maintained regardless of immediate consumer needs or wants. Environmental product attributes were in the main non-negotiable and thus 
open only to selling (or even hiding) rather than marketing. The element of long-run consumer welfare that is added by the SMC therefore was introduced here in a very different manner than that of short-run or immediate satisfaction. The small social mission company managers had decided that it was themselves (by dint of their wide experience in the area) who could adjudicate on what in fact was in the best long-run interests of society.

In contrast, CWS, the largest social mission company, had also taken account of long-run consumer interest, but did so by canvassing the opinions of these customers, as well as to some extent those of pressure groups and other external constituencies. Thus, the SMC in this context was given a more distinct marketing emphasis since the social concerns that were incorporated were customer-driven. This is close to Prothero's (1990) position on the SMC and green marketing, where the "metaneeds" of increasingly sophisticated consumers are seen as driving the SMC. Also, Kotler's (1972) original formulation of the SMC stresses the need to focus on those issues highlighted by consumer groups in order to maintain the firm's legitimacy.

Deciding what exactly is in the long-run interests of consumers or of society, and what constitutes "salutary" or "desirable" products as opposed to merely "pleasing" ones, is not however a straightforward task (Kotler, 1972; Abratt and Sacks, 1989). The attempts in the social mission companies to focus either on the beliefs of managers or of customers represent two means of attempting to do so, but clearly the NGO-collaboration route discussed in chapter 7 represents a third. Indeed, this approach might more readily accommodate the SMC, due to the way in which it opens up the firm to dialogue and 
participation with non-economic stakeholder groups. Stakeholders such as pressure groups can act as an important moral voice or social control for corporations and therefore can play a critical role in determining and effecting the long-run interests of consumers and society (Smith, 1990; Fineman, 1997). This can not only counteract corporate hegemony over discourses of rationality and truth (Tombs, 1993) but also can avoid the wholesale capitulation to (real or rhetorical) short-term consumer desires.

In the end though, the adoption of a societal marketing orientation must lead to the firm imposing some restriction on the choice of products available to consumers according to perceived social and environmental consequences (Prothero, 1990). As Kotler (1972) himself contends, merely "pleasing" products should be phased out despite their appeal to consumers. Certainly the restriction of consumer choice was an element intrinsic to the Plus Group initiative, as some respondents (albeit a minority) attested: "being in the Group isn't about offering choice"... "customers wont have a choice", etc. Corporate respondents other than those in the small social mission companies though chose not to articulate issues of consumer choice in this way, contrasting as they did with prevalent discourses of consumer sovereignty evident both in the present sample, as well as elsewhere (see Du Gay and Salaman, 1992; Knights et al., 1994). Hence, if prompted to consider the impacts on consumer choice, they would fall back on any available evidence suggesting that in fact they were driven by "customer pressure" from "out there" despite the general perception of limited green purchase behaviour. This pressure might be as little as a few letters - "if we saw ten letters on the same subject, we would respond to that" one manager claimed - since this could be 
mobilised to represent an important and growing consumer trend, just as it could an insignificant but bothersome campaign co-ordinated by one of the "fanatical" pressure groups. In many respects this reluctance to acknowledge the full implications of the SMC owes much to the role of morality in green marketing practice. It is to this that the discussion now turns.

\subsubsection{Morality in Green Marketing Practice}

As it was stated in chapter 2 , the main concern of this study with respect to green marketing practice is the moral meaning experienced, understood and communicated by organization members. This issue was examined mainly by assessing whether individuals involved in the green marketing process experienced any sense of morality, and if so, what form this might take, and to what subject it was applied. Overall, the findings revealed that in relation specifically to the environment, the personal moralities of those involved in the green marketing process were, on the whole, under-stated and pragmatic. Respondents were found to bear much more similarity to Fineman's (1997) "unimpassioned" UK automotives executives than Drumwright's (1994) "evangelistic" and "crusading" US middle managers. As Fineman (1997) himself suggests, this implies that in terms of Kohlberg's (1969) six stages of cognitive moral development (see section 2.3.1), the majority of respondents here had generally failed to move beyond the "conventional" level of morality, i.e. they lived up to what was expected from them in their role (stage 3), and fulfilled legal or accepted obligations (stage 4).

This lack of moral engagement crossed most organizational categories and hierarchical levels, with the most notable exception being, perhaps 
unsurprisingly, at the top of the small social mission companies. Here managers appeared to have moved towards a more "principled" level of moral development (Kohlberg, 1969), where the environmental welfare of others was seen as a powerful motivating force (stage 5), and organizational decision making was ruled by their own personal ethical principles (stage 6). These personally committed executives however had generally eschewed the 'strong' culture approach of disseminating their green values through the organization, thereby allowing individual members to develop relatively dispassionate and detached moralities of their own. Therefore, to a large extent, greening in these companies was significantly meaningful in a moral sense only to a small slice of the organizational membership (in this case the top). Equally, in more conventional companies, only small pockets of moral engagement with the environment were in evidence, such as where individuals had direct contact with green pressure groups, since this could prompt feelings of unease or guilt in relation to the environment (Fineman, 1996).

Overall then, few individuals amongst the total sample of respondents presented the process of marketing environmental products (compared to nonenvironmental products) as distinctly moral in character, beyond conventional standards of acceptable behaviour. In this sense, they were exhibiting what Bird and Waters (1989) refer to as a "moral muteness" both within, and towards, their work. Organization members tended to avoid any description of their work in terms of "idealistic" or "emotive" moral talk, and spoke instead of the environment as a technical or functional area of individual expertise or corporate cost/value, such that individuals involved were "just doing my job" in much the same way as those in other areas. Whilst for a minority (mainly 
social mission company managers), the involvement of their organization in providing products with green features was an "added bonus", most were relatively untouched. As one manager summed it up: "I don't think that making environmental products will make you more environmentally conscious ... you do tend to get blasé about it."

Clearly this represents a situation broadly in line with the 'shallow' as opposed to the 'deep' green marketing paradigm - a not altogether surprising finding. But if individuals themselves were not morally engaged in green marketing, where were notions of morality observed to impact upon the green marketing process? Communications, codes, and products were identified as the main subjects of moral meaning in this respect.

\section{Communications}

At a basic level, green communications were the most common issue to which moral considerations of both a positive and a negative nature were considered relevant. In the case of the former, the incidence of distinctly moral themes in green communications was not extensive, and tended to correlate positively fairly well with the perceived importance of corporate morality amongst the various audiences - and negatively with the likely possibility of subsequently attracting unwanted attention from more hostile stakeholders. Hence, organizations had generally opted to develop different communication vehicles for different audiences, allowing for careful control of the moral claims and perspectives carried by them. Where moral themes were used, the most common strategy was to focus on the corporation rather than on individual products, a finding consistent with Iyer et al.'s (1994) investigation of green 
television advertisements in the US. Indeed, to the extent that green communications might be regarded as part of the wider process of maintaining legitimacy with, and/or fostering trust from, various stakeholder groups, this was much as expected. Specific products might well represent good examples for the organization to use in justifying its legitimate status in society, but it is at the level of the organization (Gray et al., 1993), industry (Patten, 1992; Symmons and Wynne, 1993; Tombs, 1993) or institutional framework (Puxty, 1986; Irvine and Ponton, 1988) that self-legitimation is likely to be primarily centred.

In terms of moral concerns over questionable green claims, many individuals highlighted the importance of being 'ethical' in this respect, although most organizations appeared to exhibit little consistency in this area, with some claims being policed far more vigilantly than others. Indeed, the attention accorded by managers to the ethics of certain claims was to some extent a function of the level of attention accorded by those stakeholders perceived as the most important and powerful. Hence, questions of morality again entered the marketing process in response to threats to legitimacy. As a result however, ostensibly 'ethical' questions were frequently experienced as exercises in appeasement or conciliation. One particularly graphic example of how the truthfulness of green claims tended to be viewed in terms of legitimacy rather than ethics emerged in an environment-related meeting at one of the sampled conventional companies. It had been discovered that the company's environmental policy statement had been printed on paper which contained an incorrect, and by all accounts accidentally printed claim, stating that the paper used was of $100 \%$ recycled material when in fact it was actually 
of a lower percentage. The small, but in PR terms potentially very dangerous, possibility that the mistake would be discovered put the managers responsible into a position where there was perceived to be no choice but to destroy the originals and re-print them; this despite the obvious environmental (and economic) cost. However, so critical was the issue of perceived credibility, that questions of right or wrong were hardly even surfaced in the discussion.

\section{Codes of Practice}

In addition to claims, most of the organizations in this study had adopted (or were in the process of adopting) some kind of code of practice in relation to the environment - the Plus Group itself being one such example. Some managers spoke proudly then of having introduced environmental criteria into standard product development procedure, supplier assessment, or even product managers' job descriptions, suggesting a successful dissemination of environmental responsibility and duty. Whilst this most certainly had given rise in some instances to pro-environmental marketing behaviours, in many cases this represented little more than another box to tick, or another form to fill in. In practice then, these codes and criteria appeared to have had limited, if any, affective impact, in that they failed to provoke any palpable sense or feeling of environmental responsibility on the part of individuals. As Kohlberg (1969) shows, the adoption of codes reflects a cognitive morality based on social accord and system maintenance rather than personal engagement and belief. In fact, in the main, deontological moralities of duty, responsibility and obligation were directed not towards the environment, but towards the organization, profession, individual leaders, or to those with which the organization participated in economic exchanges (Watson, 1994a). 
Accordingly, the most compelling normative behavioural standards were those such as "responsible management", a "professional approach", "quality", and "customer service" or "customer expectations". In the case of marketing exchanges, this was centred around notions of loyalty, reciprocity and trust between customers and suppliers - although as it was argued in chapter 7 (section 7.3), such rationales were often used to justify or even mask power differentials and resource dependencies (Pfeffer, 1981; Frances and Garnsey, 1996). Overall then, the attitudes and emotions associated with the working moralities of organization members focused more on job-related and cultural characteristics which impacted upon personal and organizational performance than they did on more abstract social issues such as the environment which were divorced from the immediate economic realm of the organization, (Jackall, 1988; Dahler-Larsen, 1994; Watson, 1994a).

\section{Products}

Any lessening of the lifecycle environmental impacts of products through recycling, waste minimisation, packaging reductions, energy efficiency, etc. was generally seen as a 'good' thing by respondents in this study. However, it was mainly only in the top ranks of the small social mission companies that there was any clear assertion that this subsequently established these products as morally augmented in the way that ethical consumers might see them (see section 2.3.2). In the majority of cases, such products were seen as no different conceptually from other product offerings: their green credentials were simply a matter of quality, differentiation or added value in the same way as other product attributes might be. Moreover, it was generally regarded as important that products with green attributes were not depicted within the company as 
"ethical", or even "green" products, because of perceived resistance from various sub-cultural interests, particularly in the context of the powerful myth of green product failures detailed in section 8.2 above.

For most respondents then the 'good'-ness of marketing outcomes was mainly good in the sense that all improvements in product quality were good. However, even this was dampened somewhat by respondents' resigned acceptance that few measures were beyond criticism in some respect. This equivocality regarding environmental impacts, and the nebulous nature of the 'best' environmental solution was a cause of concern for many interviewees, particularly since they hampered organizational efforts to communicate in a positive fashion to external stakeholders. Analysis of the environmental impacts of products and processes in the larger, more bureaucratic, organizations weighed heavily on techno-rationalist, utilitarian, approaches such as environmental impact assessment and cost-benefit analysis, where "measurability" was the critical feature. Indeed, for these companies, development of mechanisms for the measurement, or "auditing", of environmental impacts was the basic first step in a responsible approach to green marketing, for ambiguous outcomes were seen as clouding any consideration of right or wrong. Hence, much faith (and in many cases, resources) were invested in the development of more sophisticated assessment instruments and analyses, such as product lifecycle assessment etc. Clearly, for policy to be decided, not only did lines had to be drawn somewhere, but they also had to be defensible to various stakeholders. Indeed, evidence suggests that, at present, LCA's have been primarily used to defend existing products against regulatory and competitive pressures rather than to actually develop 
greener processes or products (ENDS Report 264, 1997:20).

In contrast, the social mission companies could be seen to have rather less faith in rational, quantified analyses of environmental impacts, stressing more deontologically-based arguments of what was, or was not, acceptable in principle. As one manager argued it: "we're trying to demonstrate the principle ... calculation [of environmental impacts] would be a bit meaningless, even if you could work it out." Nonetheless, the Plus Group initiative was widely seen as attractive since it removed the inherent ambiguity of assessment altogether by transferring the responsibility (though also the control) over to an external organization much trusted by the public (Mendleson and Polonsky, 1995). As many of the Plus Group interviewees commented, the appeal of the alliance to businesses was that it set measurable and achievable targets and it provided the means by which clear statements of fact could be made and defended. Thus the inclusion of less quantifiable issues into the remit of the alliance, such as indigenous people's rights etc., did not have to become a concern of corporate managers since it was covered by the guarantee of the quality of "management" at source forests (see section 7.4.3).

\subsection{Conclusion}

In this chapter, the findings relating to the three organizational approaches as presented in chapters 5-7 have been developed in order to present a clear analysis of green marketing practice and of its moral dimensions. The case study research strategy has allowed for a fairly detailed examination of these issues in a diverse, albeit limited, series of contexts. A number of important conclusions can be reached. 
First, the picture that emerges in relation to green marketing practice is principally one of adaptation in the face of continued change. At the time that fieldwork was conducted, the environment had clearly fallen down the economic, political and social agendas since its peak in the late 1980s/early 1990 s, and yet firms were still experiencing continued (and in aggregate terms, increasing) internal and external impetus towards greening. Also, the past strategies and tactics of firms - and the success or otherwise of these - were observed to have had substantial impact both on the market, and on the current strategies pursued by the sampled organizations. This had led to the emergence of "muted" greening strategies, whereby environmental impacts were addressed, but products were positioned according to conventional product attributes; in a minority of cases, focus strategies which targeted small green niches were also viable.

Second, it was mainly only in the small social mission companies that very profound green attributes were offered, and where environmental credentials were a crucial determinant of whether a product would, or would not, be marketed. In other cases however, improved environmental features were found to be gradually introduced, often accompanied by increasingly sophisticated systems of assessment. These might be regarded as constituting a 'deep' green and a 'shallow' green approach respectively.

Third, it can be argued that green marketing strategies and tactics are best conceptualised from an interactionist perspective, i.e. with the organization viewed in terms of internal and external relationships. In particular, the stakeholder model of the firm, and to a lesser extent the networks model, have 
been shown here to provide effective frameworks for locating green marketing theory.

Fourth, by adopting an analytical position on green marketing moralities, it was possible to identify a range of moral positions adopted by respondents and organizations. This suggests certain limitations, even dangers, in assuming that the existence of green marketing behaviours is strongly linked to any particular ideological position. There could perhaps though be a correlation between the type of behaviours, or the manner in which they are conducted, and some combination of the organization's moral stance, the working moralities of its managers, existing cultural knowledge, and the enacted power and influence of stakeholders. Specification of this model might be a fruitful avenue for research in the future.

Fifth, it was also found that the implementation of aspects of the societal marketing concept could take a variety of forms. It was shown that the firms taking greatest consideration of social issues did so with an approach that appeared to bear little relation to conventional marketing theory, i.e. their orientations were principally of the "selling" variety. The approach most in touch with Kotler's (1972) original SMC conceptualisation then was that which concentrated on transparency and enhancing consumer sovereignty. However, it was shown that a more appropriate approach to societal marketing might be that which, in addition to transparency, was also based on more open dialogue and participation with polities without economic stakes in the firm.

Finally, moralities in the green marketing process were shown to be under- 
stated in relation to the environment itself, but less so in terms of procedures more directly related to personal and organization performance. The most compelling normative standards appeared to be qualities such as professionalism, responsibility, quality, customer service, and trustworthiness, etc. Even marketing elements such as communication, codes of practice and products were shown to be subjected to only limited moral engagement. Nonetheless, the ambiguity of the morality of marketing outcomes in relation to the environment remained a thorny problem, particularly for those companies attempting to utilise utilitarian ethical rules. Assessment by independent external organizations appeared to offer considerable promise in this respect.

In the next chapter, the morally penurious picture of green marketing presented here will be examined in more detail, with attention focused on how and why this might have occurred. In order to do so, the concept of amoralization is revisited and its dimensions are set out more explicitly. Following this, the possibility of the opposite process of moralization is considered, and the potential for a 'moralized' green marketing discussed. 


\section{Amoralization, Moralization, and the Management of Morality in Marketing}

\subsection{Introduction}

In the last chapter, it was shown how morality in green marketing tended to be considerably under-stated in respect to the environment itself, and was more commonly associated with directly job-related and performance-related procedural norms. This cultural dynamic tended also to be translated into marketing artefacts - policies, products and promotions for example - under the moderating influence of both real and constructed stakeholder influences. Overall, this represented something of a denial of moral meaning - a phenomenon referred to here as amoralization. Basically, what is meant by amoralization is the removal of moral meaning from the green marketing process, or from the objects of green marketing; essentially it concerns the phenomenon whereby something (here, green marketing) is rendered an amoral subject. We can therefore present a definition thus ${ }^{73}$ :

Amoralize v. 1. To interpret amorally; to point the moral neutrality of; to not make (an event, etc.) the subject of moral reflection. 2. intr. To refrain from moral reflection. b. trans. To change the condition or aspect of (a person or thing) by amoral discourse or reflection. 3. To make amoral.

\footnotetext{
${ }^{73}$ This definition is revised from the entry for "moralize" in The Shorter Oxford English Dictionary on Historical Principles (Vol. II), 3/e, p.1355.
} 
The discovery of amoralization is regarded as perhaps the single most significant finding of this study. Therefore, the purpose of this chapter is to explore it in more detail. However, although it has not been explicitly discussed until now, we can also posit that where the result of cultural activity has the opposite effect - namely that morality is given meaning in the context of green marketing - this can be regarded as moralization. In this chapter then, the analysis of the results presented in chapters 5-7 will continue with an examination of these two constructs - amoralization and moralization - as they occurred in the context of green marketing. The main purpose is to explain how and why each might have occurred, and what their consequences might be. Following this, some of the broader implications of amoralization and moralization will be discussed such as to build up a picture of how morality in marketing generally might be managed. Finally, the main findings of the chapter are summarised and some conclusions are set out.

\subsection{Amoralization in Green Marketing}

One of the principal findings of this study is that the underlying cultural dynamics of green marketing can conspire to re-frame the environment as an amoral subject. Hence, organizational actors were found to be rendered largely morally indifferent to the process and goals of green marketing, except where it impinged upon existing, job-related, working moralities (Fineman, 1996). These working moralities contained within them implicit notions of what was right or wrong to do and think in the organization, centring on accepted, though often unstated, normative standards (Jackall, 1988; Bird and Waters, 1989; Watson, 1994a; Fineman, 1996). Hence, referring to this phenomenon as "amoralization" is not meant to imply that it renders organizational life 
morally neutral overall, but rather that it removes any possibility of the environment and environmental product attributes in particular being accorded any moral meaning over and above other organizational issues which may lack such explicit social import.

There was evidence of this phenomenon, in various guises and to varying degrees, in all three organizational approaches considered in chapters 5-7. It was most pronounced in the case of the conventional companies since green marketing here was almost entirely castrated from moral meaning. In the collaborative organization, morality tended to be successively stripped from green marketing as communication proceeded from the pressure group through to the sub-cultural groups with greatest market interaction; as such it remained in certain small areas. In the social mission companies, amoralization was evident, although far less complete than in the other cases; in the main it related primarily to rank and file employees. It is the intention of this section to examine the amoralization phenomenon as observed in the study with respect to four considerations: agents (who is involved?), processes (what is involved?), causes (why does it occur?), and consequences (what are the implications of its occurrence?).

\subsubsection{Agents of Amoralization}

In almost all cases, the process of amoralization was observed to occur partly as a result of the behaviour of those individuals and groups who were the principal environmental reality definers in their organizations. These are referred to here as agents of amoralization. Such agents could be seen to abet and sustain this process either by active participation (such as in the cases of 
BTC and the business-NGO collaboration), or by passive acquiescence (as was more the case in the social mission companies); either way it was not entirely clear whether all of those involved were wholly conscious either of the process, or of their role in $\mathrm{it}^{74}$.

In chapter 5 , it was shown that the main agents of amoralization were policy entrepreneurs, or environmental champions. It was clear that these individuals had taken on their role of establishing the environment within the marketing process more for career advancement than for satisfaction of personal green commitment. However, having been charged with such a responsibility, these individuals were observed to have developed a certain recipe for responding to it successfully. In many ways, these tactics centred around processes of amoralization since the policy entrepreneurs clearly regarded a more moral conception of the environment, and of green marketing, to be a serious handicap in advancing the green agenda within the organization. Generally shallow and instrumental environmental values were thus disseminated through the organization.

A similar picture emerged in the case of business-NGO collaboration in chapter 7. In this case though, the process of amoralization was not so much

\footnotetext{
${ }^{74}$ Asking respondents directly would of course have been one solution. This however was not deemed appropriate in many instances due to the fact that interviews were deliberately focused on environmental rather than ethical issues as discussed in section 4.5.2. Only where respondents themselves had already brought up questions of morality was this approach taken. In other situations, more elliptical reference was made to the amoralization process. This could help to gain insight without unduly steering respondents, or otherwise prompting desirability bias.
} 
associated with a single set of individuals but with series of such groups, or subcultures, involved across the range of organizations in the alliance. It was shown in the previous chapter that this feature can be regarded as a consequence not only of marketing being an inherently boundary spanning function, but also of green marketing being an interactionist phenomenon. Accordingly, the role of these agents in shaping amoral conceptions of the environment can be ascribed to a more general process of cultural mediation. Successive iterations of such mediation were shown to gradually strip away layers of moral meaning. Again, this was seen by those involved as a necessary process in order to implement the alliance and to thereby develop greener marketing practices and procedures. In this instance, the process of amoralization did not appear to be complete, and moral conceptions of the environment were observed to remain to some extent in certain quarters.

In the social mission companies considered in chapter 6 , environmental reality definers were observed to occupy more senior positions. Whilst this might be expected given the greater historical preoccupation with social issues in these organizations, it could also be partly a result of their smaller size and hence less extensive division of labour (i.e. they would not be in a position to employ a lower ranking specialist). Here, the agents of amoralization did not necessarily deliberately enact the process which led to amoralization in the same way as in the other cases. However, their focus on individual moralities did have the effect of failing to morally engage other organization members with respect to the environment. In CWS, this occurred due to the prominence given by policy entrepreneurs to consumers' environmental convictions rather than those of staff. Hence, the moral imperative was to respond to consumer 
desires rather than engender internal green commitment. In the other social mission companies, this occurred due to the strong attachment of environmental guardianship to the companies' leaders, provoking little moral commitment (and even sometimes resistance) from the lower ranks.

\subsubsection{Processes of Amoralization}

Having identified who was driving the amoralization dynamic, it is possible now to turn towards considering what elements it actually consisted of, namely the processes of amoralization. In the three previous chapters, various aspects relating to the cultural dynamics of green marketing have been shown to have contributed to this phenomenon. Because of the exploratory nature of this study, these have been set out so as to maximise their utilisation to the analysis, or story, associated with the particular approach considered in each of the chapters, rather than to develop one single line of theory. However, from the point of view of developing a more general theory, it is important now to group these dynamics into the more coherent super-ordinate categories and constructs which have emerged from second-order analysis of the data, and more abstract reasoning. In all, four principal processes of amoralization can be identified: constructing morality boundaries; appropriating and privileging of discourses; mobilising narratives; and personalizing/depersonalizing. Whilst it is recognised that there might be some overlap between certain elements of these constructs, they are regarded as the most effective and compelling way to capture the main essence of the amoralization process. 


\section{Constructing Morality Boundaries}

This element has two main strands: the circumscription of distinctly moral conceptions of the environment into certain (minority) polities or arenas; and the construction of the environment as manifestly different from ethical issues amongst other (majority) polities. In the case of the former, we might cite the examples of: a) the senior managers of the social mission companies who articulated strong personal moral positions regarding the environment; b) subcultures of environmentally committed individuals in the case of the business-NGO collaboration; and c) the proposed existence of only small niches of consumers exhibiting green purchase behaviour. In each instance, considerable evidence was presented to suggest the personal moral engagement of these individuals or groups in at least some aspects of the green marketing process. In the case of the first two examples, there appeared to be no attempt on the part of those morally committed to the environment to impose their own frames of reference on others, or to go to any significant lengths to seek "converts" among their organizations. Changes in the values of green converts might though be regarded as an important element in developing forms of green marketing based upon personal moral conviction and cultures of social responsibility (Drumwright, 1994; Menon and Menon, 1997). In this sense then, these environmental protagonists were content with eliciting pro-environmental behaviours from others rather than seeking more fundamental attitudinal change. In the case of green consumers, moral issues were repeatedly argued to be of little, or no, concern for the majority of the public - again bounding morality within the minority.

With respect to the second strand in the construction of morality boundaries, 
the case of BTC was perhaps the most striking example revealed by the study. Here, there were clearly explicit attempts by environmental policy entrepreneurs to define the environment as conceptually distinct from ethical issues. Environmental product issues were presented very much as technical issues which demanded technical solutions (such as LCA and auditing systems) rather than moral reflection. Fineman (1997) suggests that this may be indicative of a general correlation between initial organizational interpretations of the environment and the particular profession or function of corporate environment guardians. Hence the interpretation of the environment in technical terms at BTC (and the reluctance to ascribe moral denotations) could be expected given that the principal environmental protagonists in the company were technical and quality managers. Equally, in CWS the perception of the environment as a "consumerist" issue could be linked to its guardianship within the corporate marketing section; and so on. Whilst there is no reason to suggest that this would necessarily exclude the environment from the moral arena in the long term, it might clearly act as an important element in the wider process of amoralization should moral issues be perceived as problematic introductions into the cultural knowledge of the organization.

\section{Appropriating And Privileging Of Discourses}

The appropriation of techno-rationalist discourses of "commercial logic" to describe environmental product issues, coupled with the subsequent privilege accorded to these discourses over emotional, ethical, or otherwise "woolly" ones was probably the single most effective process of amoralization observed in this study. This was most evident in the cases of conventional and collaborative organizations, where only "rational", "business" arguments of 
customers, profits and costs were considered useful in promoting the green agenda. Hence, the prevailing rhetoric of "win-win" solutions countered any possibility of discussing competing motives for greening except in a few instances. An upshot of this was the wholesale avoidance of various semantic suspects, i.e. words and phrases with implicit connotations of morality, and which therefore might have been regarded with suspicion by power interests within the organization. Accordingly, terms such as sustainability, biodiversity, ecology, etc. were shrewdly omitted from the management lexicon in order to avoid alienating important marketing constituencies. Occasionally they would surface in marketing communications to certain stakeholders, although usually in a form which promised little in practice, and invited little real emotion work from internal audiences, e.g. claims to be "committed to the principle of sustainable development" (as opposed to the substance). Bird and Waters (1989:73) refer to this as the "moral muteness" of managers, such that:

(Managers) talk as if their actions were guided exclusively by organizational interests, practicality, and economic good sense even when in practice they honour morally defined standards codified in law, professional conventions and social mores.

In the present study, this appropriation of commercial discourses in the conventional and collaborative organizations was supported by considerable symbolic action on the part of environmental protagonists. Much of this centred on attempts at image making so as to ensure that there was congruence between the commercial themes of the green message and the "responsible 
management" image of the messenger. In the main, cultural norms dictated that successful green project management relied on a "professional" and "businesslike" manner, untainted by the stereotypical image of the environmentalist "crank". Hence the discourse-based amoralization required other cultural cues, such as dress, appearance, and manner, in order to garner authenticity and credibility.

Whilst these are certainly important empirical findings, they should not be regarded as wholly surprising. There is a large and growing body of literature associated with the critical role of language and discourse in constructing social and organizational realities (e.g. Morris, 1949; Pettigrew, 1979; Barley, 1983; Watson, 1994a). Watson (1994a) for example argues that language is the principal vehicle for the transmission of culture and values. Accordingly, he argues, corporate discourses shape the way that individuals within them think and behave. Morris (1949:214) contends that "sharing a language with other persons provides the subtlest and most powerful of all tools for controlling the behaviour of ... other persons to one's advantage." Managers in the current study clearly recognised that the political realities of organizational life were such that appropriating existing discourses was likely to be far easier than establishing new ones, based on contentious or challenging concepts. Hence, one of the BTC environment protagonists spoke of "certain words and phrases and approaches that turn the locks in a business". This acquisition of appropriate categories of language was thus seen as a crucial prerequisite for positive participation in marketing decision making procedures. Moreover, by doing so, these individuals also acquired along with the language, the accepted ways of doing of things of these groups, and their implicit value implications 
(Pettigrew, 1979; Watson, 1994a). Accordingly, the privileging of the discourse of "commercial logic" and its concomitant denial of a language or vocabulary of morals in relation to the environment meant that cultural frames of reference for green marketing in the conventional and collaborative organizations were bereft of moral meaning beyond conventional standards of honesty, responsibility, etc.

\section{Mobilising Narratives}

The third mechanism of amoralization concerns the way that green marketing protagonists tended to associate the environment with existing organizational and marketing narratives. In the case of BTC, this took the form of 'narrative surfing, such that various narratives were mobilised according to the exigencies of the situation. In other cases, green marketing was principally embedded in a single potent narrative, such as in CWS where a tradition of consumerism was readily invoked to explain greening initiatives.

Such activities can be regarded as attempts to apply meaning to organizational events through symbolic activity. This might be both for the purpose of personal sensemaking, as well as (more critically) to exercise some degree of control over organizational interpretations of events in order to reduce uncertainty, increase control and to manage expectations (Brown, 1995b; Brown and Ennew, 1995; Weick, 1995). In the conventional and collaborative organizations, green marketing protagonists could thereby facilitate relatively uncontroversial perceptions of greening, principally by abating any perceptions of newness, difference, or strangeness on the part of influential groups within and outside of the organization. Critically, this meant that the 
environment could be presented as a "normal" activity for the business, denying any necessity for further moral engagement on the part of organizational members.

Clearly, traditions and past events were prime subjects for mobilisation in this respect since they represented relatively uncontested events which could be selectively plucked from the organizational history in order to provide meaning and legitimacy to events which might otherwise be open to unfavourable interpretation (Rowlinson and Hassard, 1993). Moreover, this in turn could also be regarded as contributing to further cementing and reenforcing the cultural values expressed by the chosen narratives (Wilkins, 1983; Brown, 1995a).

In the social mission companies, further sense-making activity could be observed in the manner that narratives of difference were at times demobilised. Whilst sometimes executives in these companies chose to illustrate how distinct they were from conventional organizations, in relations with external constituencies such as suppliers and competitors they chose to play down any difference that might be projected upon them on account of their ethical stances. Hence the mobilisation or otherwise of the narrative of difference tended to be used judiciously in order to support different readings of the moral culture by various stakeholders.

\section{Personalizing/Depersonalizing}

Amoralization was also driven in part by the extent to which greening initiatives were constructed as being owned by, or a personal campaign of, 
certain individuals. For example, the agents of amoralization in BTC and the Plus Group alliance worked hard to engineer consensus within their organizations that environmental considerations were a corporate matter rather than a personal crusade on their part. Hence, "it wouldn't work if I was to get people to push it to me, they are the ones that have to integrate it into their day-to-day jobs". Whilst this was certainly motivated in the main by pragmatic desires to distribute functional responsibility for environmental issues more widely, and to avoid being personally overburdened with low status operational issues, this lack of personal identification with greening initiatives was also seen to be congruent with establishing a "responsible management" approach which focused on "rational" and "objective" commercial criteria. Clearly, a personal crusade on the part of environmental protagonists might have suggested that they were driven by an overtly moralistic agenda which may have damaged the credibility of themselves as managers, and of the projects that they worked on.

Such depersonalizing and distancing of the individual from organizational events is a common feature of life in large organizations (Bate, 1984; Jackall, 1988). By denying "ownership" or guardianship of the environment, individuals could avoid difficult, even "dysfunctional" interpersonal confrontation which might have been marked by ideological exhortations rather than rational decision making (Bate, 1984; Bird and Waters, 1989). As such, concerns over the possible threat to organizational harmony as well as the personal dangers of being marginalised from the "real" strategic business of marketing products probably contributed significantly to amoralization in this way (Bird and Waters, 1989). 
In contrast, the dynamics observed in the social mission companies suggested contradictory findings. Here, the company's mission was generally seen to be very strongly tied to the ethical beliefs of certain key senior executives:

There are people in the management group who only consider what good you can do - and you do need them. I mean, I'd always go the commercial route. And its all right for them - the (senior manager 1) and (senior manager 2) of this world - they don't have a commercial responsibility like I do.

Significantly though, this personalization of green marketing initiatives also seemed to contribute to the process of amoralization, albeit in a different manner to that in the case of depersonalization in the other organizational forms. Here, the perceived attachment of morality issues to the crusades of senior executives appeared to some extent to prompt those further down the hierarchy to morally disengage, and even at times to sabotage, what they saw as disruptive incursions into their established working practices (Collins and Ganotis, 1973). Overall then, it could be said that depersonalization contributed to amoralization by denying the possibility of personal moral responsibility for the environment at all, whilst personalization contributed by attributing moral responsibility too narrowly. To negate amoralization (or to promote moralization) it would seem likely that some level of personalization would have to be engendered, perhaps by ensuring that moral responsibility (rather than simply functional responsibility) was spread more widely through the organization. This is considered in more detail in section 9.3.

What this relatively brief, but instructive, analysis of the amoralization process 
suggests then is that environmental protagonists tend to conspire both consciously and unconsciously, deliberately and accidentally, to render themselves as agents of amoralization. This role involves a complex pot pourri of signification and meaning management, making use of an array of symbolic and semiotic resources (such as rhetoric, stories, metaphor, and difference). But why do these phenomena occur? And why are the cultural and symbolic domains the scene of this activity? To answer these questions it is necessary to explore a little deeper into the social and moral terrain of the organization.

\subsubsection{Causes of Amoralization}

To some extent, in course of this and the previous four chapters, some discussion of the causes of amoralization has already been made. Broadly, it has been suggested that two main themes can be identified as driving the amoralization dynamic. First, in the conventional and collaborative organizations, the building of bridges of understanding between environment protagonists and vital marketing constituencies in and around the organization (e.g. company buyers, brand managers, etc. as well as suppliers and customers) could be seen to necessitate tactics of amoralization in order for green marketing projects to be seen as a legitimate part of corporate activity. In these contexts, overtly moral perspectives on social goals were generally viewed as likely to arouse suspicion, and therefore those associated with such displays susceptible to marginalisation from the marketing process. Second, in the social mission companies, the focusing of moral sensibilities regarding green marketing at very senior levels was observed to have occasioned processes of amoralization in respect to less senior employees. Morality regarding social goals in these instances simply failed to be disseminated 
through the organization as part of the socialization process and the transmission of corporate values. However, although these two themes construct some kind of rationale for the dynamics observed, they are not in themselves the root causes. Indeed, it is important to explore these causes in more depth so as to provide some explanation for why these differences between organizational types occurred. To this we must turn to a yet deeper level of analysis and consider fundamental elements of organizational structure, culture, politics and power.

\section{Organizational Structure and Systems}

Given that amoralization was a more powerful force in the conventional and collaborative organizations than in the social mission companies, it could be argued that one of its root causes might be the impact of size and structure. Mintzberg (1983) for example suggests that large, divisionalised, bureaucratic organizational forms are less likely to promote morally rooted social responsibility positions than small single-product firms (as were three out four of the social mission companies). Jackall (1988) too shows how routine, bureaucracy and comparmentalization in the life of large organizations help to shape taken for granted frameworks on issues outsiders might find morally pertinent (such as the environment), whilst concentrating managers' moral consciousness on their own personal survival and success. Bureaucracy thus can not only distance the individual from the moral consequences of their actions, but may also prompt him/her to conspire to remain so, in order to avoid any "unnecessary" obfuscation of their much valued (and rewarded) "rationality" in decision making (Jackall, 1988). 
The work of Bauman $(1989,1993)$ provides a strong theoretical basis for such a consideration of amorality in bureaucratic organizations, and his appears to be a growing influence on the business ethics literature (see for example Parker, 1997; ten Bos, 1997). Ten Bos's (1997) treatment of Bauman's work highlights certain features of his critique that appear to relate substantially to the process of amoralization considered here. To use Bauman's terminology, amoralization might be regarded as a process whereby decisions are rendered morally indifferent or adiophoric, i.e. "of a kind on which ethical authorities do not feel it necessary to make a stand" (1993:30). Ten Bos (1997) contends that Bauman sees morality in bureaucracies as driven by obedience and abidance to rules (a "technocratic morality"), rather than by moral autonomy, reflection, or impulse. Furthermore, he illustrates how Bauman explains this by reference to three features of bureaucratic organization: the imposition of moral distance ("denial of proximity"); dehumanisation ("effacement of face"); and the reduction of total persons to traits (e.g. customers are reduced to their needs, workers to their productivity, etc.). The upshot of this is that morality becomes instrumentalised with respect to the goals of the organization, rendering a complete disregard of the moral substance of the actual goals themselves (ten Bos, 1997).

Clearly, these arguments present important theoretical insight into the causes of amoralization in green marketing. Bureaucratic systems in themselves tend to render marketing goals - whether environmental or not - beyond moral concern, since it is the techno-rationalist process of marketing which is viewed as the arbiter of good and bad. If due marketing process (signifying "commercial logic") has been observed - that is, if customers have been 
researched, products tested and sold, and sales data analysed - then the bureaucratic ethic has been followed. This extends also to more societal marketing orientations whereby the social concerns of customers (e.g. CWS) or pressure groups (e.g. the Plus Group) are simply incorporated into this process, without questioning the moral basis of the process itself, or of its goals. Accordingly, the processes of amoralization described above can be said to constitute attempts by green marketing protagonists to expedite their projects into this process, thereby legitimising them in the eyes of their colleagues, superiors, and certain other stakeholders. However, the fact that this phenomenon was observed across organizational forms suggests that other factors might also have been at play. Indeed, evidence for the amoralization dynamic was most strongly evident in respondents' depictions of the cultural and political processes accompanying green marketing initiatives. Therefore these are likely to have had a direct bearing on this process of legitimation and are worthy of discussion in themselves.

\section{Power and Politics}

In the social mission companies, political manoeuvring in relation to green marketing activities was not substantially in evidence (particularly in the smaller firms), although there was evidence of resistance to greening at middle management and shop floor levels. In both the conventional and collaborative organizations however, the context for implementing green marketing initiatives was generally depicted as highly political, with various constituencies contesting the necessity and/or appropriateness of greening, as well as negotiating the form and level of its potential impact on marketing and marketing-related activities. This did not seem to be seen as particularly 
surprising by respondents, since large organizations were widely regarded as being characterised by subcultural interests in respect to any organizational activities, developments or change. The introduction of environmental considerations into marketing activities however was to some extent seen as bringing with it additional political baggage due to perceptions of "woolliness", "crankiness" and, perhaps most importantly, potential "commercial suicide" in relation to greening. Consequently, political activity often focused around manipulation of symbols and language to play down these undesirable associations, resulting, as has been shown, in significant amoralization.

Pfeffer (1981) argues that the control over organizational language and symbols rests with those wielding the most political power and influence. His argument is based upon the assumption that language and symbolism are used to rationalise and legitimate decisions which have already been reached on the basis of power. Accordingly, language and the ability to use political symbols contribute only marginally to the development of the power of various organizational participants, but are important in the exercise of such power. Many other studies of leadership and organizational politics (e.g. Pettigrew, 1973, 1985; Brown, 1994, 1995b) also either explicitly or implicitly support this view by highlighting how already powerful individuals and groups within organizations manage to use language and symbols to generate support for their objectives and quiet opposition.

In terms of such organizational power and influence, striking differences were observed between the green marketing protagonists in the social mission 
organizations and those in the conventional and collaborative organizations. Put simply, the former were in a position of power whilst the latter were not. In the social mission companies greening was, to varying degrees, a "strategic contingency" (Salancik and Pfeffer, 1977), i.e. it was essential to the achievement of the firms' prominent social goals. Therefore, its guardians had substantial scope for how it was to be incorporated into marketing goals and processes. Hence, CWS corporate marketing executives could be said to have chosen the discourse of "consumerism" in which greening was couched since it both supported and reinforced their goals of establishing a more committed marketing orientation into the company. Equally, executives in the smaller companies could be said to have chosen to frame the environment as a matter of personal moral conscience since it helped to shape their own self-images of concerned, even moralistic, individuals.

In the other organizational forms, the environment was clearly not such a strategic contingency: it was not only viewed as contributing to increased uncertainty, but also lacked any centrality or critical dependence for other functions or activities (Salancik and Pfeffer, 1977). This was exacerbated by its lack of ownership by the marketing function itself (Drumwright, 1994). Therefore green marketing protagonists had little choice but to re-frame the environment such that it was consistent with existing organizational symbols, narratives and discourses in order to render it a legitimate corporate subject. Hence green meanings were pragmatically attuned to commercial logic and the profit motive by dis-association with symbols of morality (personal attachment, discourses of morality, etc.) and association with symbols of rationality (narratives of customer care traditions, discourses of responsible 
management and commercial logic, etc.). The role of power relations was perhaps most evident in the collaborative case, since it was clear that the green marketing project in question represented a more critical strategic activity for the pressure group than it did for most of the corporate partners. Consequently, the pressure group was more dependent on the companies' resources than vice versa, leading to a situation of only limited power on the part of the WWF. This helps to explain why the companies' amoral version of the project came to dominate over the pressure group's more emotional and moralistic perspective.

Power then not only allows for the manipulation of language and symbols but is further consolidated by it. Knights and Morgan (1991) use a Foucauldian analysis to show how the legitimacy of discourses is bound up in the power relations which characterise the social context in which they are used. The authors show how categories (here, examples might be "responsible management" and "responding emotively") are constructed in particular social contexts; and once subjects have come to understand the world in those terms, organizational practices emerge which re-enforce this 'truth'. Organizational knowledge is thus institutionalised by creating a 'way of seeing' which reproduces its own truth effects. What this means is that once powerful groups begin to subjectively characterise the environment in a certain way (or are perceived as doing so), this particular interpretation tends to become institutionalised over time as objective truth.

\section{The Role of Culture}

This avowedly political process associated with the implementation of green 
marketing practices in organizations also indicates a considerable role for subcultural interests and identities in shaping the development of amoralization. Many of the processes of amoralization in the case of conventional and collaborative organizations were driven by needs to bridge subcultural divisions due to power deficiencies on the part of environment protagonists. Hence, whilst working moralities might be regarded as sunk within subcultural communities and professional associations (Sinclair, 1993; Anthony, 1994), green subcultural moralities specifically appeared to be traded, suppressed, or at least bracketed, in the face of more powerful normative impulses associated with personal success, existing relationships and, above all, commercial logic (Jackall, 1988; Fineman, 1996, 1997). In the social mission companies, diversity in subcultural green moralities was allowed to flourish. Hence, marketing policy makers may have had strong environmental sensibilities, but other cultural groups continued to maintain a certain moral indifference.

Indeed, in many of the sampled companies, 'strong' organizational cultures and subcultures were perceived and presented as significant obstacles to change. Generally accepted ways of doing things within the organization, or group, were seen as prescribed, or at least frozen, and consequently tended to be observed rather than transgressed (Bate, 1984; Schein, 1992). Environmental values tended to be absorbed into existing cultural knowledge and certainties rather than elicit any kind of moral transformation of these (Fineman, 1996). Accordingly, agents of amoralization presented a kind of "learned helplessness" in the face of established cultural norms (Bate, 1984). Dahler-Larsen (1994) suggests that such barriers are likely to be grounded in 
the denial of moral autonomy promoted by 'strong' corporate cultures which reward compliance to managerial values rather than promoting a diversity of independent values and moralities. Hence, the possibility for a moralization of marketing becomes suffocated by the instinctive adherence to routinely declared and re-iterated corporate values as presented in formal company communications and literature (Dahler-Larsen, 1994; Fineman, 1996, 1997). Worse perhaps, high levels of commitment to the corporate culture can be seen even to provoke environmental irresponsibility through lack of moral reflection and dialogue (e.g. Heilbroner et al., 1972).

Finally, Christensen (1995) argues that the meaning suggested by various marketing artefacts can also contribute to a solidification of the organizational culture. He contends that the products of the marketing process have an important role to play in shaping and re-enforcing organizational identity, that in effect, external marketing communications also communicate substantially internally, i.e. they are "autocommunicative". Therefore, the muted green communications revealed by this study, where emotional and moral appeals were downplayed or non-existent, might be regarded as both a product of the power interplays within the organization which rendered legitimate amoral conceptions of the environment (Phillips and Brown, 1993) but also as a signal to employees that this was indeed is the most appropriate frame of reference to adopt (Christensen, 1995).

\subsubsection{Consequences of Amoralization}

The discussion surrounding amoralization in green marketing inevitably leads to one final question: does it matter? The answer to this of course will depend 
on the perspective one is looking from, particularly whether one adopts a 'deep' or a 'shallow' green perspective (see chapter 3). This division is therefore used as a platform from which to discuss the potential consequences of amoralization.

It has been widely argued from the deeper ecology end of the green business literature that significant voluntary advances towards greener products, production processes and communications will require companies to embrace 'ethical' core values, and principles of social responsibility and sustainability (e.g. Stead and Stead, 1992; Peattie, 1992, 1995; Shrivastava, 1994, 1995a/b; Welford, 1995). The evidence of this study would tend to give partial support to this argument since, as it was shown in the previous chapter, relatively minor and incremental changes to the environmental quality of existing green products clearly predominated over the development of radical green alternatives, particularly where green moralities were unexpressed or absent. Hence the extent and profundity of greening appeared to be in inverse proportion to the degree of amoralization evident in a given organizational form. This is summarised in table 9.1 .

Certainly the senior managers of those social mission companies which marketed products to other organizations were clear in their conviction that only on the basis of a distinct moral basis would these reselling organizations offer the kind of support necessary for them to develop their radical green product and process innovations. It was argued that without such values in place, organizations would always take the simplest route, which entailed the least amount of change, whereas what the social mission companies actually 
Table 9.1: Consequences of Amoralization

\begin{tabular}{|l|l|l|l|}
\hline \multirow{2}{*}{} & \multicolumn{3}{|c|}{ Organization } \\
\cline { 2 - 4 } & Conventional & Collaborative & Social Mission \\
\hline Extent of & Basic, & Profound & Radical \\
Green & incremental & development in & developments in \\
Marketing & changes & one area & several areas \\
\hline throughout & & \\
\hline Amoralization & & Extensive & Partial \\
\hline
\end{tabular}

needed was some degree of assistance, flexibility, and patience to allow them to progress in new and unique directions.

Clearly though, what table 9.1 also shows is that some level of greening is possible without attendant morality, and fairly radical greening is possible without a unitary 'ethical' culture. Indeed, for the most part, it could be argued that to initiate fundamental greening initiatives into marketing, a distinctly moral engagement may only perhaps be necessary on the part of the strategic leader, providing they have sufficient power to do so, and the requisite political skills and dexterity with symbols to render the process legitimate. This does however call into question those prescriptions identified in chapter 3 (section 3.4.2) which suggest that a distinctly moral culture and shared environmental values throughout the organization are necessary for radical 
green marketing practices to take place. The evidence from the social mission companies would tend to refute such prescriptions, with the proviso that organization size and bureaucracy might have moderating effects.

Of course, there are important arguments from the deep ecology school that an ecocentric approach to green marketing should by definition involve not only the participation of internal and external stakeholder groups, but also their moral and affective engagement (e.g. Shrivastava, 1995a). From this point of view, the findings reported here are both disappointing and troubling: disappointing in the sense that a truly ecocentric green marketing approach according to this definition was nowhere wholly in evidence (though certainly elements of it were), even where it might be most expected, such as in business-NGO collaboration and social mission companies; troubling in the sense that few respondents actually saw this as a problem, or even a particularly important issue in itself - and of those that did, e.g. some of the social mission company managers, the problem was viewed as especially troublesome, and largely secondary to more immediate concerns of survival and progression towards existing organizational objectives.

Arguably of even more concern however is that if amoralization is an important feature of contemporary green marketing (as it is argued here), then it may also progress into other stakeholder groups and polities. This might be regarded as reasonably likely given two significant findings of the current study. First, the extent of amoralization even in the context of collaboration with environmental groups (as illustrated by the WWF Plus Group case) might be a cause of concern given that such groups might be regarded as a moral 
conscience for corporations (Fineman, 1997) or a form of ethical control in themselves (Smith, 1990). Second, the avowed intention of many sampled companies to focus communication efforts at converting external groups to the organization's environmental world-view (see section 8.2.2) might be seen as attempted hegemony, or railroading, of the environment debate such that the techno-rationalist conception of the environment comes to predominate.

For example, the development by a number of the firms in this study of environment education material for schools might be seen as a barrier to developing a more pluralistic green agenda which recognises and encourages a diversity of views and perspectives, rather than simply that of large, powerful, commercial organizations. It is the prospect of this excrescence of environmental amoralization on the part of economic institutions that has prompted green marketing critics such as Gorz $(1980,1989)$ to argue for the need to limit the scope of the economic system and to liberate environmental questions and policies from it altogether.

In contrast, from a reform or shallow green perspective, the existence and extent of amoralization indicated here would not necessarily be viewed with consternation, provided that environmental improvements in marketing process and outcome could be continued. However, the limits of voluntarism in this respect should not be under-emphasised (Newton and Harte, 1997). The results here indicate that motives of ethics and social responsibility for greening initiatives are rarely claimed by executives other than those from social mission companies. This suggests that coercion from powerful stakeholders, such as pressure groups and regulators, mediated by politically 
adept and professionally ambitious internal champions, is more likely to encourage greening of marketing practices than any voluntary, moral impulses or principles (Fineman and Clarke, 1996; Fineman, 1996, 1997). Indeed, there is concern, as many of the respondents here indicated, that the introduction of "emotive" and "woolly" moral arguments can hinder rather than facilitate the passage of organizational greening, once the firm has been prompted into action. This is clearly a fundamental pragmatic of organizational life that the deep ecology school will have to better accommodate in order to develop prescriptions for eco-change actually based on the realities of corporate life.

\subsection{Moralization}

Having to date described a fairly morally penurious view of contemporary green marketing practice, the final section of this chapter seeks to explore how morality has been, and potentially could be, injected into this practice, or to continue in a consistent lexicography, how marketing moralization might be effected. In particular, proposals for programmes of culture change as introduced in chapter 2 (section 2.3.1) are examined; this is then used as a basis to discuss the potential for moralization in the three organizational approaches considered in chapters 5-7.

\subsubsection{Moralization Through Culture Change}

This study has been based on the assertion that marketing morality, and more specifically green moralities in marketing, can be examined through cultural phenomena, knowledge and artefacts. As it has been shown though, the more common perspective in the business and marketing ethics literatures, as well as 
in the green business literature, is that the group of elements which comprise the organization's culture can be changed in some way to effect greater morality in marketing practice. Whilst the data collected here only related in a fairly minor way to explicit culture change programmes, they do suggest that rather than culture being a platform for marketing moralization, it commonly acts as a significant barrier to such. Indeed, in the conventional and collaborative approaches, widespread perceptions of cultural immutability had prompted little attempt at culture change, such that where positive green values were in evidence, they were not disseminated more widely through the organization. Green behaviours had though in some cases been encouraged within these organizations, both at a corporate level (e.g. greening of products and processes) and at a more local level (e.g. re-use and recycling of office supplies), although the values articulated to legitimate such activities were in the main of an instrumental nature. Hence, "in practice, enacted 'ethical' moralities are essentially pragmatic, well-attuned to the organization's profit interests, and expediently adjusted to different subcultural interests," (Fineman, 1996:492). Culture change then, where it was apparent, might be regarded as relatively superficial and symbolic rather than representing fundamental shifts in organizational values, beliefs and assumptions. Even where greening was seen to be supported by socially responsible cultures, these were existing and stable cultural forms rather than changing ones (Drumwright, 1994; Fineman, 1996)

In the social mission companies, more attention had been given to green cultural change, although it was recognised that cultural uniformity in this respect would be extremely difficult and potentially even undesirable. The arguments relating to the difficulty of changing cultures are by now well 
rehearsed in the organization and management studies literature (e.g. Martin, 1985; Nord, 1985; Siehl, 1985; Ogbonna, 1992; Anthony, 1994). Indeed, there is by now little empirical evidence in the literature which provides wholesale support for the claim that culture can indeed be managed. Cultural pragmatists that do support the proposition tend to conceive of culture in relatively superficial terms - i.e. in relation to behavioural change - whereas those that refute it tend to define culture in deeper and broad terms - i.e. in relation to values and beliefs (Martin, 1985; Ogbonna, 1992). That 'greener' or more 'ethical' cultures can be developed also lacks empirical substantiation beyond accounts which are either brief and anecdotal (e.g. Smart, 1992; Bernstein, 1992) or self-reported (e.g. Chappell, 1994). Accordingly, the use of culture management to improve corporate ethical and environmental behaviour has (belatedly) begun to be seriously questioned (Sinclair, 1993; Dahler-Larsen, 1994; Fineman, 1996; Newton and Harte, 1997). Sinclair (1993:68) for example concludes that:

The lessons from research are that you meddle with the organizational culture if you've got little choice, lots of resources, and lots of time - a combination of circumstances, some would argue, rare enough to render the approach irrelevant.

The issue of cultural uniformity being in itself undesirable has also been considered to some extent in the green and ethical business literatures and is clearly an important one (Sinclair, 1993; Dahler-Larsen, 1994; Fineman, 1996). Dahler-Larsen (1994) for example argues that drives for cultural unity reward conformity rather than the very autonomy that is crucial for a sense of 
morality to exist. Fineman (1996:493) points to the possibility that attempts at cultural unity might railroad the green agenda according to managerial perspectives, thus driving out fundamental ecological principles of inclusion and democracy:

If it is axiomatic that ethical environmentalism is built upon respect for others, it follows that any ethical developments in an organization's culture should acknowledge the range of values and feelings within that organization.

Ultimately, both Fineman (1996) and Sinclair (1993) suggest that sub-cultural identities are perhaps the principal repositories of organizational moralities as well as potentially offering the best route for influencing or activating moralization. The proposition that subcultural values and norms act as both highly integrative organizational bonds and important influences on organizational micro-behaviour is well established in the organization studies literature (e.g. Martin and Siehl, 1983; Van Maanen and Barley, 1985; Martin, 1992; Anthony, 1994). It is possible that a positive moralization effect could thus be fostered by encouraging surveillance, dialogue, and critique between these subcultural groups, prompting "ethical discourse and dialectic as well as conflict" (Sinclair, 1994:69). Starkey (1998) re-enforces this argument with the contention that moral development in organizations requires factionalism and dissent in order to promote learning. Hence the role of management becomes one of surfacing conflicting values, unleashing the moral commitment of subcultures, and from this forming synthesis and mutual coexistence rather than authoritarian ideological control (Sinclair, 1994; Starkey, 1998). 
This non-unitary perspective certainly appears to relate strongly to the empirical realities of many of the organizational contexts studied here, displaying as they did clear sub-cultural attachments and moral frames of reference. Indeed, affective moralization of the environment was clearly bounded into certain subcultural arenas, suggesting significant potential for this approach. However, processes of amoralization were found to occur partly through the power differentials evident between these arenas and other subcultural factions. Therefore, whilst the prescriptions for moralization based on differentiation might be regarded as correct in identifying the positive effect of embracing difference, encouraging moral dialogue and surfacing awareness of conflicting moral perspectives, what they lack is any explicit identification of the importance of the relative power of these subcultures. Indeed, this power is critical in shaping the discourses in which these differences, dialogues, and perspectives can actually be articulated (Pfeffer, 1981; Phillips and Brown, 1993). This is particularly true in the case of specific issues (the environment) and specific processes (marketing of products) since these are likely to be strongly associated with certain occupational subcultural groups which are perceived to hold guardianship over them.

Dahler-Larsen (1994) takes the criticism of organization cultures in relation to moralization yet further. For him, the failure is not just in the superficiality and authoritarianism of unitary cultures, but also in the substitution by culture of internal corporate attachments in the place of society as the 'sacred' moral realm. Hence, even feeling engagement through sub-cultural associations 
would be deficient for the establishment of true morality since "nothing less than society itself is the source and objective of morality" (Dahler-Larsen, 1994:7). Starkey (1998) counters this by emphasising both the fragmentation of broad societal identities and moralities, and the rise of corporate visions/missions which aspire towards social goals. In the current study, such social aspirations appeared to transfer most significantly from 'out there' annoyances into the moral order of the organization when attached to powerful internal polities (such as the company's leadership). Without this internalization, social concerns tended to regarded as customer, pressure group, or media issues which needed to be 'managed' in line with profit interests rather than being any source, or subject, of morality in themselves (Jackall, 1988; Bauman, 1993).

Finally, a 'moralized' green marketing might also be expected to involve some formal communication of that sense of morality to internal and external audiences through marketing artifacts such as products and promotions. Products are in themselves important repositories of meaning, particularly in terms of brands, and as cultural artifacts in their own right can communicate key aspects of morality. This would account for some of the claims by the social mission company leaders that the mere existence of their products in the marketplace was a representation and legitimation of a certain social ideology (see section 6.3.1). However, division of labour and employee specialisation means that internal constituencies often remain ignorant of the moral import of product offerings. This can be accentuated when communications intended for external audiences are seen by employees (as is often the case of advertising, labelling etc.) and the values articulated are incongruent with those held or 
experienced internally (Gilly and Wolfinbarger, 1998). Hence, whilst moralization might be expected to be accompanied by greener products and promotion, it is unlikely that these alone would be sufficient to prompt it.

\subsubsection{Routes to Moralization}

Overall then, the evidence from this study suggests that any moralization of green marketing practice is likely to be significantly easier in companies with explicit social missions than in those without. Perhaps surprisingly, this does not however appear to be moderated by the centrality of the mission to the firm's marketing strategy. Indeed, of more importance than the mission per se are the systems of structure, culture and power within the organization that determine the legitimacy of an internal moral discourse around greening. And in these companies, such systems appeared to be reasonably supportive of intimations of moralization provided that the integrity of the leaders' convictions was (or was perceived to be) genuine (Fineman, 1997), and that it did not conflict too much with existing perceptions of organizational values (Gilly and Wolfinbarger, 1998) or expectations for personal cultural autonomy (Martin, 1992). To the extent that the social mission companies in this study were apparently increasing their attention to culture management techniques, there is clearly a case for suggesting that moralization might become in this way more expansive than was in evidence at the time of fieldwork. This would indicate a subtle shift away from a focus solely on reforming external publics towards also seeking to convert internal subjects (Mintzberg, 1989). Whether this would then impact negatively on the cultural and moral autonomy of employees would probably depend on the manner in which it was introduced, i.e. whether more attention was focused on opening up dialogue across 
existing sub-cultural divisions, selecting-in environmentally-concerned employees at the recruitment stage, or 'educating' all employees to internalise the company's (unitary) values. Given the previous discussion, it might be expected that this list represents decreasing potential for true moralization.

In theory, the impact of external stakeholders on marketing practice, such as in the case of the Plus Group, might also appear to represent some possibility of moralization. Such interaction might be viewed as an opportunity for the introduction of new cultural knowledge into the marketing process in the form of vocabularies, stories and symbols with more explicit moral resonance. This can prompt organizational learning through the development of alternate "frame-breaking" perspectives (Menon and Menon, 1997). In practice however, this effect was found to be limited, most notably because of the moderating effect of the subcultural buffer provided by environmental protagonists. This does not however mean that a more radical approach from pressure groups would be met with greater success in effecting moralization since firms have been shown to be generally unwilling or unable to accept the legitimacy of concerns raised by more aggressive green stakeholders (Fineman and Clarke, 1996). Indeed, informants here emphasised the superior appeal of the WWF as a partner compared with, for example Greenpeace and Friends of the Earth, since the WWF was perceived as run by "nice people" who were more "conservative", "responsible" and "willing to work with business" than other "fanatical" pressure groups. It remains then a fine balance for collaborative stakeholders to maintain in working with companies: too much overt moralizing and they will be rejected; too little and companies are unlikely to be fundamentally challenged at a cultural level. 
Finally, in the case of the conventional companies, the most promising potential route to moralization would be through one or more powerful senior executives adopting a crusading policy entrepreneur role either from within, or interacting with, marketing and related departments. Given sufficient political power and influence, and assuming that others perceived the stance as genuine, such a role could have substantial impact on organizational members' articulated green values, creating space for interpretations of greening outside of purely instrumental and commercial discourses. However, there is clearly a delicate balance to be maintained here also. Too much personalization of the environmental agenda can disaffect other organizational members suggesting that environmental protagonists might have to adopt a more 'empowerment' type approach. Critically, this form of moralization would probably need to focus on developing within some kind of subcultural community (either new or existing) and ensuring that it wielded substantial power within the marketing organization.

\subsection{Conclusion}

In this chapter, the findings relating to the three organizational approaches as presented in chapters 5-7 have been further developed, and consequently two main themes - amoralization and moralization - have been set out and explored. These themes have yet to be examined to any extent within the existing green marketing literature (nor even the broader marketing literature) and therefore the proceeding discussion should be seen as an attempt to develop new and original 'grounded' theory in this respect. A number of important insights have been made. 
First, amoralization has been defined in the current context as the removal of moral meaning from the green marketing process, or from the objects of green marketing, i.e., it is the phenomenon whereby green marketing is rendered an amoral subject. Amoralization can be seen to be an important element in the green marketing process, and was in evidence in all three organizational approaches, albeit to a varying extent.

Second, amoralization was shown to be tied to the behaviour of certain individuals and groups within the organization - usually the principal environmental protagonists - and these agents of amoralization were argued to effect this process in a number of ways. Amoralization could therefore be argued to emerge either deliberately or accidentally, consciously or unconsciously. To some extent this was seen to depend on the context faced by these individuals and groups.

Third, four principal processes of amoralization were set out in order to capture the range of cultural dynamics which were observed to contribute to this phenomenon. These conceptual categories - constructing morality boundaries, appropriating and privileging of discourses, mobilising narratives, and personalizing/depersonalizing - should be regarded as important emergent variables in explaining the amoralization dynamic. In particular, they help to illustrate how cultural and symbolic elements within the marketing organization have a substantial effect on the moral meanings that are subsequently constructed and communicated within it.

Fourth, the causes of amoralization were located within the realms of 
structure, power, politics, and culture. Whilst to some extent this might be regarded as simply reflecting the attempt to make a cultural analysis in this study, the citing of these findings within important streams of organizational and management literature adds strength to the veracity of the findings. Indeed it could be argued that it is the failure of the mainstream literature in marketing, business ethics and green business to take critical account of cultural and political dimensions that has to date weakened their theoretical insight and practical relevance (Gladwin, 1993; Brownlie et al., 1994; Brigley, 1995; Newton and Harte, 1997).

Fifth, the consequences of amoralization were discussed, and although it was argued that even extensive greening of the marketing process could potentially take place without strong moral engagement being widely shared throughout the organization, the degree of amoralization was shown to vary inversely with the extent of green marketing programmes observed in the case organizations. Other potentially negative consequences were identified, such as the subsequent lack of support for radical green innovation, and the possibility of the process of amoralization extending into other stakeholder groups, which might limit the potential for a pluralistic green agenda. Therefore, from a 'deep' green perspective, the consequences of amoralization would be regarded as profoundly negative, although from a 'shallow' perspective this might not necessarily be the case. Indeed, the avowed necessity of amoralization in order to introduce green issues into the marketing process as expressed by some respondents in this study might suggest that the positive effects outweigh the negative consequences. 
Sixth, the possibility of moralization of green marketing was also discussed in the context of these findings. Moralization was defined as the opposite to amoralization, namely as the phenomenon whereby green marketing was interpreted morally, subjected to moral reflection, or made morally relevant in some way. The potential for culture change to realize moralization was examined and found to be wanting due to factors such as the difficulty of managing deep layers of culture, the subsequent exclusion of moral autonomy and contrasting values, and the failure to focus morality outside of the organization. An approach to culture management which encourages subcultural attachments and moralities to develop and flourish was identified as superior, although it was argued that the findings of this study suggest that power differentials between these factions might be a critical feature in determining the success of such approaches.

Finally, possible routes to moralization were examined for each of the three organizational approaches considered in this study. It was argued that this process was likely to be easiest in the social mission companies, and the potential for an expanded morality in such contexts was set out. The collaborative approach was shown to have some potential, although in practice this had not been extensively realized in the case selected. Lastly, in the conventional companies the possibility for greater moralization was set out in terms of the political power and influence of policy entrepreneurs - where this was higher, the potential for moralization was argued to be greater. 


\section{Conclusions}

\subsection{Introduction}

This study has sought to investigate morality in marketing. It has done so by looking primarily at one particular form of marketing, broadly defined as 'green marketing', and by conducting an exploratory, qualitative, case studybased empirical investigation aimed at understanding the practice of green marketing, and at revealing its moral dimensions. In this final chapter, the extent to which this study has achieved its aims will be discussed, and the significance and contribution of the study will be assessed. This is the main subject of the section immediately following. In the sections following this, these conclusions will be further developed in the context of respectively: the managerial implications of the findings, the possible limitations of the study, and recommended directions for future research. In the final section, a summary of the main contributions of the thesis are presented.

\subsection{Main Conclusions}

It is proposed that a number of important findings have emerged from this study, and that these have important implications for theoretical development in the marketing, business ethics, and green business literatures. In this section these conclusions are set out, and their implications discussed. 


\subsubsection{Marketing as a Moral Domain}

We can conclude first that marketing is indeed a moral domain, and it is possible to forward three main reasons for this, as explained in chapter 2. First, marketing has been subjected to some very important moral critiques, and the extensions of marketing theory represented by the development of 'societal marketing', 'green marketing' and 'social marketing' that have been formulated in response, suggest that the goals of the marketing system are not necessarily purely economic in nature. The acknowledgement of social and environmental outcomes can therefore make the incorporation of moral dimensions into marketing fairly explicit. Second, descriptive studies of 'ethical decision making' in marketing have shown that marketing decisions are beset by moral considerations and criteria, and that marketing decision makers are indeed likely to at times proceed through some form of process of ethical reasoning. Third, the ethics of product and organization have been used as a form of product augmentation (both positive and negative), and can clearly form an aspect of the consumer's product evaluation.

Even though it is possible to say then that marketing is a moral domain, it does not follow that it can also be said that marketing is necessarily either 'ethical' or even 'moral'. It is not necessarily 'ethical' because this is in itself a value judgement, and to say so would presuppose that marketing had been evaluated according to certain ethical rules, principles or criteria. This may indeed be the case, but it does not follow from the observation that marketing is a moral domain. It is also not necessarily 'moral' because, within the terminology of this thesis, to say so would suggest that those involved in the marketing process did so with an understanding of, feeling for, and use of, moral 
arguments, categories, vocabularies, and sensibilities. The inquiry of whether indeed such moral meaning is a part of the marketing process is an empirical question, and is the principal one investigated during the course of this thesis.

\subsubsection{Green Marketing Theory and Practice}

In order to explore moral meaning in marketing, it was decided to focus the main part of the thesis specifically on green marketing, i.e. marketing activities whereby some account had been taken of dimensions relating to the natural environment. In chapter 3 it was shown that green marketing theory offered a variety of perspectives on morality, rather than any single consistent perspective. These could be broadly categorised in terms of the 'deep' versus 'shallow' greening distinction employed by the green business literature.

Following empirical examination of three very different approaches to green marketing, it was found that few individuals in organizations saw environmental features as strong product augmentations, and green marketing strategies were on the whole muted. In the main then, environmental quality was seen either in terms of maintaining legitimacy in the eyes of government and non-government organizations, or in terms of a personal goal of the firm's leadership. Hence, overall it was argued that, in practice, green marketing could be best understood as a process composed of a network of internal and external interactions. Critical internal interactions were observed to occur between those charged with the responsibility for introducing greening into the marketing process and other important marketing constituencies within the organization. Critical external interactions were observed to occur between the 
focus organization and its suppliers, competitors, customers, governmental and non-governmental organizations, other industry network members and other stakeholders. Accordingly, it was argued that green marketing could be modelled fairly successfully utilising stakeholder theory and the networks model of marketing exchange, both of which defy any atomistic view of the individual firm. These are considered to be fruitful avenues for subsequent development of green marketing theory. Moreover, although these theoretical perspectives on green marketing could be said to have little to say in themselves about morality, their basis in relationships makes possible a moral view of green marketing through their particular theoretical lenses.

\subsubsection{Cultural Dynamics of Green Marketing}

The cultural dynamics of green marketing have been explored and analysed in some depth during this study, particularly to the extent that they reveal the moral terrain of the organization. Whilst these dynamics clearly varied according to the particular organizational context focused upon (see chapters 5-7), common themes relating to the manipulation of language, symbols, and images could be observed. These dynamics are considered to be important explanatory variables in understanding the green marketing process. Moreover, these were further argued to contribute significantly to a process of amoralization in green marketing - i.e. a phenomenon whereby moral meaning was removed from the green marketing process. Accordingly, in chapter 9 super-ordinate categories of those dynamics which contributed substantially to amoralization were set out, namely constructing morality boundaries, appropriating and privileging discourses, mobilising narratives, and personalizing/depersonalizing. 
Amoralization was shown to be a significant feature of green marketing in the organizational environments studied, and it can be postulated that it might be a significant feature of marketing generally. Clearly then, the cultural analysis of organizations can be a very effective means of identifying and examining important new dimensions of morality in marketing. In the main, amoralization was shown to arise from the need or otherwise by environmental protagonists to control the organizational meaning and shared frames of reference that attended green marketing projects and policies. The rationale for this was generally presented in terms of ensuring a relatively uncontested and uncontroversial introduction of new policy. Moreover, it was argued that fundamentally, the existence or otherwise of this need depended on variables such as levels of organizational size and bureaucracy, the relative power resources of various organizational and extra-organizational actors and subcultures, and the perceived strength or 'stickiness' of the perceived extant culture of the relevant group or groups. The addition of these cultural and political dimensions to the study of morality can be seen then to be vital in terms of developing new insight based on the realities of organizational life. Clearly, this is an area and approach which demands further attention in the future such as to provide for a more informed body of knowledge in relation to morality in marketing and business more generally.

\subsubsection{Moral Meaning in Marketing}

In general then, for those actually engaged in the marketing process, moral meaning in marketing practice was found to be rather under-developed, both cognitively and affectively. Such a possibility was shown quite dramatically by this study, with the goals of marketing only rarely acknowledged as being 
morally meaningful by these individuals and groups. More commonly, the moral domain of marketing appeared to be focused on internal subjects, and in particular on role-related behavioural norms, codes of practice, and rules regarding organization members' personal and organizational success. This has important implications for how marketing might be conceptualised and theorized. In particular, the finding that the introduction of social and environmental goals into marketing practices does not necessarily follow from, or elicit, any form of moral transformation within the marketing organization, suggests that the revised forms of marketing discussed in chapter 2 constitute, in practice, only very minor departures from the largely technicist perspective of traditional marketing theory. Hence, the expansion of the goals of marketing to include social and environmental features does not necessarily constitute a new marketing paradigm, but merely an extension of the existing one.

Moreover, the possibility that marketing might actually be a territory for amoralization seems to be one that is rarely, if ever, confronted within the marketing literature. However this is clearly an issue that is critical to an understanding of morality in marketing, and surely should not be overlooked in this way. If it is truly possible to envisage a 'moral', not least an 'ethical' marketing, then the true role and nature of moral meaning simply can not be ignored, and it would be as well to begin with an understanding of the practical moral realities of marketing rather than idealistic prescriptions of how it 'should' be. Thus, the identification of marketing amoralization might be regarded not only as an important addition to the knowledge of marketing practice but also as a critical element to be incorporated into models and 
prescriptions of, for example, societal marketing and marketing ethics, as well as green marketing.

\subsection{Managerial Implications}

So what lessons and insights, if any, do the results of this study provide for managers involved in green marketing, or for those otherwise interested in rendering the marketing process more 'moralized' or more 'ethical' in its execution? There are in fact a number of findings here which can be seen to have implications for marketing management, and specifically for green marketing management, and which can be seen as having practical import for organizations, managers, and employees.

First, it can be argued that successful green marketing programmes need to take account not simply of the individual firm but of the network of internal and external interactions which might potentially impact upon any given programme. Accordingly, the role of the green marketing protagonist is likely to be in some sense political, suggesting that a high degree of interpersonal and political skills might be required for successful achievement of the project's goals. From an organizational point of view, the alliance approach to green marketing might be seen to provide a suitable framework in which to manage these various relationships more successfully. Moreover, alliances with stakeholders and network members appear to offer substantial potential for marketing solutions which can take account of 'deep ecology' principles of mutuality, interdependence, and reciprocity. Critical in this respect appears to be the level of attention accorded to managing the cultural variations between 
collaborating organizations and the congruity of bonds between the actors, resources, and activities linked by the project.

Second, for managers wishing to introduce greening into relatively unsympathetic, even hostile marketing contexts, the evidence from this study suggests that some degree of management of the moral meanings attending greening may be necessary. Whist there must clearly be doubts as to the longterm advisability of completely amoralizing the greening process, it can not be doubted that some degree of amoralization appears to successfully facilitate the introduction of greening initiatives into the organization. The way to do this, as it has been shown, is to focus on the realms of language and symbolism, thus necessitating some considerable dexterity with symbols on the part of environmental protagonists. Again, those elements identified as the processes of amoralization (section 9.2.2), as well as the contextually-specific cultural dynamics detailed in chapters 5-7, might be consulted for guidance on possible avenues for achieving this.

However, from an environment point of view, such amoralization might be seen as potentially harmful to the green agenda, and certainly in terms of 'deep' greening, should probably be avoided. In order to introduce more fundamental and lasting greening initiatives then, a more 'moralized' process would be recommended. By the same token though, the scope and potential for individuals to manage morality - and especially to enhance moralization appears to be largely pre-determined, not so much by their own ability, but by existing organizational characteristics. The third main implication then relates to the rather limited possibilities for morality to be injected meaningfully into 
the marketing landscape. Basic structural factors such as organizational ownership, governance, size, and degree of bureaucratisation might have considerable impact on the potential in this respect and hence managers wishing to introduce greater moralization into a large, highly bureaucratised, joint stock company governed by professional directors, and owned by institutional shareholders are likely to be faced with far more extensive obstacles than those confronted with the same task in a small, entrepreneurial, social mission co-operative. This is not to say that the former will necessarily be less ethical than the latter, only that the awareness and attention to morality in general is likely to be lower. Moreover, levels of amoralization and moralization appear to be highly contingent on the cultural and political context in which the marketing process is enacted, and it is highly likely that only by grasping the cultural and political dynamics at play in any given situation can the manager begin to effect any kind of meaningful moral transformation of marketing.

This is not to suggest that the manager can, or even should, attempt to change the culture of the organization. The discussion in the previous chapter suggests that this might be not only extremely difficult, but possibly even counterproductive to the objectives intended. Clearly, attempts at cultural homogeneity and unity have less potential for moralization than those which recognise the existence of subcultural differences and their attendant power differentials. The fourth, and final, implication then is that managerial knowledge of the prevailing political and cultural landscape of the organization rather than of an ideal landscape is critical for developing insight into which dynamics might be fruitfully employed either to introduce green or 
social projects in a relatively uncontested fashion, or - somewhat more challengingly - introduce a more moralized version of these projects.

\subsection{Limitations}

It was described in chapter 4 how a grounded, inductive, case-study based methodology provided considerable epistemological potential for exploratory empirical work in the area of marketing and morality. Clearly though there are certain limitations with the approach adopted here, and with the way in which it has subsequently been applied. Nonetheless, this is only to be expected there is, as it was argued, no one true and perfect method; what is important though is that the limitations of that which has been used are made explicit, and in this way the reader can judge for their self the veracity of the resultant theory. The purpose then of this section is to discuss the main limitations of the study, and this shall then form the basis of the following section which seeks to set out possible directions for further research.

Perhaps the most common, and strongest objection to case study work utilizing a relatively small number of cases is that the findings are too contextually specific, and thus limited in their generalizability to other cases and circumstances. Certainly, it can not be said that the findings reported here are representative of business organizations generally, and there can be little argument that the conclusions reached can only claim validity with any certainty for the particular cases selected as the sample for this investigation. However, by the same token, there is no reason to suggest that the results presented here are unique or unusual. It has been attempted to develop a comparative case sample exhibiting substantial variation in context, and 
therefore by developing theoretical propositions and constructs to a degree of abstraction sufficient to encompass these variations, it is possible to hold considerable confidence that they could be readily applicable to other situations, and to other organizations. Moreover, it is the depth of insight gained through qualitative, interpretive research that is its major strength, particularly in exploratory research, and it is inevitable that some level of breadth has to be compromised to achieve this given the bounded conditions of this study. However, to enhance confidence in the representativeness of the findings, and to test for generalizability, further cases should be investigated in the future.

There are also questions of generalizability in relation to the attempt in this enquiry to study morality in marketing by focusing on one particular form of marketing, namely green marketing. In this sense, there must again be limitations in the extent to which the findings relating to green marketing can be readily assumed to have implications for other forms of marketing, and for marketing more generally. It is important to recognise therefore that at this early stage in theory development, such implications can only be suggested rather than guaranteed. Nonetheless, although these results are grounded in substantive data, they again have been developed to a level of abstraction that makes them potentially generalizable to situations and contexts which might occur in other substantive areas within the formal field of marketing and morality. Hence, the intention has been to generate substantive theory as a "strategic link" in the formulation of formal theory from data (Glaser and Strauss, 1967). In order to strengthen this link though, other substantive areas such as societal marketing and social marketing should be explored in the 
future, and even more conventional marketing processes such as new product development, market research, and communications should provide fertile sources of substantive data.

Although the issue of generalizability is probably the major limitation of the study, there are a number of more minor concerns. First, the main form of data collected during the fieldwork stage consisted of respondents' own impressions, thoughts and arguments. There is no guarantee that these were their 'real' opinions and although some degree of triangulation with other data sources was attempted where possible, and considerable attention was paid to reducing desirability bias (see section 4.5.2), the results reported should be viewed in the context that they might represent only the espoused convictions and insights of respondents. Also, although some account has been made of the historical context of the sampled organizations, and although data collection was performed over a 16 month period, this study does represent to some extent something of a snapshot of the field of study. The situation revealed here is very much one of a particular time and context, and historical or longitudinal research could aid substantially in developing more temporally embedded theory.

Finally, it should be recognised that the conclusions reached should not be assumed to constitute a concrete or highly 'objective' reality, but rather, it is hoped, a credible and convincing reality which has been mediated and shaped by the researcher through rigorous and imaginative analysis. Indeed, this is very much an exploratory study, and should not be regarded as the definitive account of the research field. Attention turns next then to proposals for possible future 
developments which might extend and expand upon the findings reported here.

\subsection{Directions for Further Research}

There are a number of ways in which this thesis could, and indeed should, act as a foundation for further research. First, given possible concerns over the limited generalizability of the conclusions reached, the most obvious direction for further research to take is in replicating the study in other organizations, within different contexts, and for forms of marketing other than green marketing. Ideally, the present study could also be extended longitudinally. In this way, the concepts and constructs emerging from the current study could be honed and sharpened into a more complete theory of moral meaning in marketing.

Second, it was argued in chapter 8 that stakeholder theory and the networks model of marketing exchange appeared to provide theoretical structure to the green marketing dynamics revealed in this study. This observation though requires further investigation before a systematic theory of green marketing can be developed. Moreover, it has been argued that these models principally provide descriptive and explanatory insight into green marketing. Their potential for normative or prescriptive power might also therefore be usefully explored.

Third, much of the discussion in the previous chapter opens up a number of potentially fruitful avenues for future research. This could include more systematic empirical examination of the antecedents of amoralization, as well as longitudinal research investigating in more detail the consequences of 
differing degrees of moralization and amoralization. Moreover, although discussed in some detail, possible routes to moralization were not explicitly tracked and tested during this study, and some exploration of their relative plausibility and potential for success would aid substantially in developing a theory of moral meaning in marketing.

Finally, although this study has attempted to draw together some of the disparate elements of the marketing and morality area such as the SMC, moral product augmentation and ethical decision making, further research is required in order to integrate these better. For example, how do amoralization and moralization fit into Hunt and Vitell's $(1986 ; 1993)$ model of ethical decision making? How exactly is product augmentation linked to moral meaning within the marketing process? And is it possible to predict different approaches to the SMC based on the cultural dynamics of the organization?

Clearly then, there is still much work to do before the area of marketing and morality is fully understood and conceptualised. Indeed, Glaser and Strauss (1967:40) argue that in developing theory grounded in data there can be no last word, and that ultimately, "the published word is not the final one, but only a pause in the never-ending process of generating theory." It is hoped then that this pause is at least an interesting, plausible, useful, and as far as possible a 'real' one. To conclude then, a summary of the main contributions to knowledge of this thesis is presented.

\subsection{Main Contributions of Thesis}

The main contributions of the thesis can be summarised in terms of the 
following four points:

1. The thesis has provided an in-depth empirically-based examination of green marketing - an area which previously had not been subjected to a great deal of empirical enquiry. The current study has not only attempted to formulate a moral dimension to green marketing, but has also explored the practice of green marketing across a range of different organizational contexts, and has provided new insight into process as well as content issues. This can be seen to be a valuable addition to an emerging, but previously rather unconvincing, area of literature.

2. The grounded, qualitative research method applied in this study is one that has been shown to be well-suited to the types of questions under examination, but which has been significantly under-utilised in the marketing, business ethics, and green business fields to date. As a result, it has been possible to develop unique and important insights into the area of marketing and morality, particularly in relation to the cultural dynamics of green marketing and the phenomenon of amoralization. Moreover, the careful use of this research engagement has contributed to some extent both to a redressing of the existing imbalance towards positivist, quantitative methods in the associated fields of study, as well answering some of the various criticisms levelled at much of the existing empirical work in this area.

3. The thesis has also made an important step towards developing a more rigorous utilisation of the concept of organizational culture to marketing, business ethics and green business. The more common utilisation of culture in 
these areas - i.e. as a managerial variable - has been largely discredited in the organization studies and organizational behaviour fields. Hence, the use here of culture as a means of analysing meaning within the organization reflects a move towards establishing a more informed conceptual understanding of morality within the marketing process.

4. Finally, and perhaps most importantly, by focusing on moral meaning, the thesis has addressed an aspect of marketing and morality which, although critically important, had not been previously subjected to any significant degree of scholarly investigation. Accordingly, new knowledge has emerged regarding how organization members experience, think, feel and communicate about morality in marketing. This has led to the development of important concepts and constructs which contribute to our understanding of this area, such as amoralization and moralization, and the various cultural dynamics of green marketing. If these findings succeed then in providing insight or illumination to fellow academics or to marketing practitioners - and there is every reason to believe that they shall - then the contribution of this thesis will be realized. 


\section{References}

Abratt, R. and Sacks, D. (1989), 'Perceptions of the Societal Marketing Concept', European Journal of Marketing, vol. 23 (6), pp. 25-33.

Advertising Association (1993), 'Speaking Up for Advertising', in G.C. Chryssides and J.H. Kaler, An Introduction to Business Ethics, Chapman and Hall, London.

Akaah, I.P. and Riordan, E.A. (1989), 'Judgements of Marketing Professionals About Ethical Issues in Marketing Research: A Replication and Extension', Journal of Marketing Research, vol. 26 (February), pp. 112120.

Aldag, R.J. and Bartol, K.M. (1978), 'Empirical Studies of Corporate Social Performance and Policy: A Survey of Problems and Results', in L.E. Preston (ed.), Research in Corporate Social Performance and Policy (vol. 1), JAI Press, Greenwich, Connecticut.

Alvesson, M. and Berg, P.O. (1992), Corporate Culture and Organizational Symbolism, Walter de Gruyter, Berlin.

Anderson, J.C., Hakansson, H. and Johanson, J. (1994), 'Dyadic Business Relationships Within a Business Network Context', Journal of Marketing, vol. 58, pp. 1-15.

Anderson, W.T. and Cunningham, W.H. (1972), 'The Socially Conscious Consumer', Journal of Marketing, vol. 36 (3), pp. 23-31.

Anthony, P. (1994), Managing Culture, Open University Press, Buckingham.

Ashley, W.J. (1912), 'Preface', in E. Cadbury, Experiments in Industrial Organization, Longman Green, London.

Aupperle, K., Carroll, A. and Hatfield, J. (1985), 'An Empirical Examination of the Relationship Between Corporate Social Responsibility and Profitability', Academy of Management Journal, vol. 28 (2), pp. 446463.

Bailey, J. (1955), The British Co-operative Movement, Hutchinson, London.

Bain, W.A. (1995), 'Ethical Problems in Ethics Research', Business Ethics: $A$ European Review, vol. 4 (1), pp. 13-16. 
Baker, M.J. (1994), The Marketing Book (3/e), Butterworth-Heinemann, Oxford.

Barley, S.R. (1983), 'Semiotics and the Study of Occupational and Organizational Cultures', Administrative Science Quarterly, vol. 28, September, pp. 393-413.

Bartels, R. (1962), The Development of Marketing Thought, Richard D. Irwin, Homewood, Illinois.

Bartels, R. (1967), 'A Model for Ethics in Marketing', Journal of Marketing, vol. 31 (1), January, pp. 20-26.

Bate, P. (1984), 'The Impact of Organizational Culture on Approaches to Organizational Problem Solving', Organization Studies, vol. 5 (1), pp. 43-66.

Baudrillard, J. (1981), For a Critique of the Political Economy of the Sign, English trans., Telos Press, St. Louis.

Baudrillard, J. (1983), Simulations, trans. P. Foss, P. Patton and P. Beitchman, Semiotext(e), New York.

Baudrillard, J. (1997), The Consumer Society: Myths and Structures, English trans., Sage, London.

Bauman, Z. (1989), Modernity and the Holocaust, Polity Press, Oxford.

Bauman, Z. (1993), Postmodern Ethics, Blackwell, Oxford.

Beauchamp, T.L. (1989), Case Studies in Business, Society and Ethics, Prentice Hall, Englewood Cliffs.

Beauchamp, T.L. and Bowie, N.E. (1988), Ethical Theory and Business (3/e), Prentice Hall, Englewood Cliffs.

Bebbington, J. and Gray, R. (1992), 'Greener Pricing', in M. Charter (ed.), Greener Marketing, Greenleaf, Sheffield.

Bendall, J. and Sullivan, F. (1996), 'Sleeping with the Enemy? BusinessEnvironmentalist Partnerships for Sustainable Development: The Case of the WWF 1995 Group', in R. Aspinwall and J. Smith (eds.), Environmentalist and Business Partnerships: A Sustainable Model?: A Critical Assessment of the Impact of the WWF UK 1995 Group, White 
Horse Press, Cambridge.

Berger, P.L. and Luckmann, T. (1966), The Social Construction of Reality, Penguin, London.

Bernstein, D. (1992), In the Company of Green, ISBA Publications, London.

Bibby, A. (1996), 'You'd Have to be Green to Stay Mutual', The Observer, 28/4/96, Business, p.9.

Birchall, J. (1994), Co-op: The People's Business, Manchester University Press, Manchester.

Bird, F.B. and Waters, J.A. (1989), 'The Moral Muteness of Managers', California Management Review, vol. 32 (1), fall, pp. 73-88.

Blackler, F.H.M. and Brown, C.A. (1983), 'Qualitative Research and Paradigms of Practice', Journal of Management Studies, vol. 20 (3), pp. 349-365.

Bohlen, G., Schlegelmilch, B.B. and Diamantopoulos, A. (1993), 'Measuring Ecological Concern: A Multi-construct Perspective', Journal of Marketing Management, vol. 9 (4), pp. 415-430.

Boje, D.M., Fedor, D.B. and Rowland, K.M. (1982), 'Myth Making: A Qualitative Step in OD Interventions', The Journal of Applied Behavioural Science, vol. 18 (1), pp. 17-28.

Bonner, A. (1961), British Co-operation, Co-operative Union Ltd, Stockport.

Bonoma, T.V. (1985), 'Case Research in Marketing: Opportunities, Problems, and a Process', Journal of Marketing Research, vol. XXII (May), pp. 199-208.

Brigley, S. (1995), 'Business Ethics Research: A Cultural Perspective', Business Ethics: A European Review, vol. 4 (1), pp. 17-23.

Brown, A.D. (1994), 'Politics, Symbolic Action and Myth Making in Pursuit of Legitimacy’, Organization Studies, vol. 15 (6), pp. 861-878.

Brown, A.D. (1995a), Organizational Culture, Pitman, London.

Brown, A.D. (1995b), 'Managing Understandings: Politics, Symbolism, Niche Marketing and the Quest for Legitimacy in IT Implementation', 
Organization Studies, vol. 16 (6), pp. 951-969.

Brown, A.D. and Ennew, C.T. (1995), 'Market Research and the Politics of New Product Development', Journal of Marketing Management, vol. 11 (4) pp. 339-353.

Brown, P. (1996), 'Shops Defy “Idiotic” Label ban', The Guardian, 10/9/96, p.9.

Brown, S. (1993), 'Postmodern Marketing?', European Journal of Marketing, vol. 27 (4), pp. 19-34.

Brown, T.J. and Dacin, P.A. (1997), 'The Company and the Product: Corporate Associations and Consumer Responses', Journal of Marketing, vol. 61 (1), January, pp. 68-84.

Brownlie, D., Saren, M., Whittington, R. and Wensley, R. (1994), 'The New Marketing Myopia: Critical Perspectives on Theory and Research in Marketing - Introduction', European Journal of Marketing, vol. 28 (3), pp. 6-12.

Buckley, N. (1995) , 'Co-op Adopts Ethical Line', Financial Times, 24/4/95, p8.

Bucklin, L.P. and Sengupta, S. (1993), 'Organizing Successful Co-Marketing Alliances', Journal of Marketing, vol. 57 (2), April, pp. 32-46.

Burrell, G. and Morgan, G. (1979), Sociological Paradigms and Organizational Analysis, Heineman, London.

Butcher, M. (1996), 'The Co-operative Movement: Business Relic or a Model for the Future?', Business Studies, December, pp. 25-28.

Cannon, T. (1992), Corporate Responsibility, Pitman Publishing, London.

Carr-Saunders, A.M., Florence, P.S. and Peers, R. (1938), Consumers' Cooperation in Great Britain, George Allen \& Unwin, London.

Carroll, A.B. (1979), 'A Three-Dimensional Conceptual Model of Corporate Performance', Academy of Management Review, vol. 4 (4), pp. 497-505.

Carson, R. (1962), Silent Spring, Penguin, Bristol.

Chapman, S. (1974), Jesse Boot of Boots the Chemists, Hodder \& Stoughton, London. 
Chappell, T. (1993), The Soul of a Business: Managing for Profit and the Common Good, Bantam Books, New York.

Charter, M. (1992), Greener Marketing, Greenleaf, Sheffield.

Chen, A.Y.S., Sawyers, R.B. and Williams, P.F. (1997), 'Reinforcing Ethical Decision Making Through Corporate Culture', Journal of Business Ethics, vol. 16, pp. 855-865.

Chonko, L.B. and Hunt, S.D. (1985), 'Ethics and Marketing Management: An Empirical Investigation', Journal of Business Research, vol. 19, pp. 339359.

Christensen, L.T. (1995), 'Buffering Organizational Identity in the Marketing Culture', Organization Studies, vol. 16 (4), pp. 651-672.

Chryssides, G.C. and Kaler, J.H. (1993), An Introduction to Business Ethics, Chapman and Hall, London.

Churchill, D. (1984), 'Why Boots is Trying Harder', Financial Times, 27/2/84, p.12.

Churchill, D. (1986), 'The Rochdale Pioneering Spirit Adrift Among the High Street Store Groups', Financial Times, 24/5/86, p.5.

Clarkson, M.E. (1995), 'A Stakeholder Framework for Analyzing and Evaluating Corporate Social Performance', Academy of Management Review, vol. 20 (1), pp. 92-117.

Clutterbuck, D., Dearlove, D. and Snow, D. (1992), Actions Speak Louder (2/e), Kogan Page, London.

Collier, J. (1995), 'The Virtuous Organization', Business Ethics: A European Review, vol. 4 (3), pp. 143-149.

Collins, J.W. and Ganotis, C.G. (1973), 'Is Social Responsibility Sabotaged by the Rank and File?', Business and Society Review/Innovation, Autumn, pp. 82-88.

Corey, E.R. (1993), 'Marketing Managers: Caught in the Middle', in N.C. Smith and J.A. Quelch (eds.), Ethics in Marketing, Irwin, Homewood.

Cramer, J. and Schot, J. (1993), 'Environmental Comakership Among Firms as a Cornerstone in the Striving for Sustainable Development', in K. 
Fischer and J. Schot (eds.), Environmental Strategies for Industry, Island Press, Washington.

Dahler-Larsen, P. (1994), 'Corporate Culture and Morality: Durkheim-Inspired Reflections on the Limits of Corporate Culture', Journal of Management Studies, vol. 31 (1), pp. 1-18.

Davidson, H. (1993), 'Copycat Rivals Scent Success as Body Shop Feels the Squeeze', Sunday Times, 15/8/93, pt.3, p.6.

Davidson, M. (1992), The Consumerist Manifesto, Routledge, London.

Davis, J. (1991), Greening Business: Managing for Sustainable Development, Basil Blackwell, Cornwall.

Davis, J.J. (1991) 'A Blueprint for Green Marketing', Journal of Business Strategy, vol. 12 (4), pp. 14-17.

Davis, J.J. (1992), 'Ethics and Environmental Marketing', Journal of Business Ethics, vol. 11, pp. 81-87.

Davis, J.J. (1994), 'Good Ethics Is Good for Business: Ethical Attributions and Response to Environmental Advertising', Journal of Business Ethics, vol. 13, pp. 873-885.

Davis, K. (1973), 'The Case For and Against Business Assumption of Social Responsibilities', Academy of Management Journal, vol. 16 (2), pp. 312322.

Deal, T.E. and Kennedy, A.A. (1982), Corporate Cultures, Addison-Wesley, Reading.

De George, R.T. (1990), Business Ethics (3/e), Macmillan, New York.

Dembkowski, S. and Hanmer-Lloyd, S. (1994), 'The Environmental ValueAttitude-System Model: A Framework to Guide the Understanding of Environmentally-Conscious Consumer Behaviour', Journal of Marketing Management, vol. 10 (7), pp. 593-603.

Devlin, J.F., Ennew, C.T., Hull, A. and Sherman, L.A. (1996), 'Ethics and the Church: Should God be Sold or Found?' paper presented at the 1996 Marketing Education Group Annual Conference, University of Strathclyde. 
Diacon, S.D. and Ennew, C.T. (1996a), 'Can Business Ethics Enhance Corporate Governance? Evidence from a Survey of UK Insurance Executives', Journal of Business Ethics, vol. 15 (6), pp. 623-634.

Diacon, S.D. and Ennew, C.T. (1996b), 'Ethical Issues in Insurance Marketing in the UK', European Journal of Marketing, vol. 30 (5), pp. 67-80.

Dibb, S., Simkin, L., Pride, W.M. and Ferrell, O.C. (1994), Marketing: Concepts and Strategies (2/e European), Houghton Mifflin, Boston.

Dillon, P.S. and Fischer, K. (1992), Environmental Management in Corporations: Methods and Motivations, CEM, Medford, Mass.

Dixon, D.F. (1992), 'Consumer Sovereignty, Democracy, and the Marketing Concept: A Macromarketing Perspective', Canadian Journal of Administrative Sciences, vol. 9 (2), pp. 116-125.

Dobson, A. (1996), 'Environmental Sustainabilities: An Analysis and Typology', Environmental Politics, vol. 5 (3), pp. 401-428.

Donaldson, T. (1982), Corporations and Morality, Prentice-Hall, Englewood Cliffs.

Donaldson, T. and Preston, L.E. (1995), 'The Stakeholder Theory of the Corporation: Concepts, Evidence and Implications', Academy of Management Review, vol. 20 (1), pp. 65-91.

Dooley, R.S. and Lerner, L.D. (1994), 'Pollution, Profits, and Stakeholders: The Constraining Effect of Economic Performance on CEO Concern with Stakeholder Expectations', Journal of Business Ethics, vol. 13, pp. 701-711.

Drumwright, M. (1994), 'Socially Responsible Organizational Buying: Environmental Concern as a Noneconomic Buying Criterion', Journal of Marketing, vol. 58 (3), July, pp. 1-19.

Drumwright, M. (1996), 'Company Advertising With a Social Dimension: The Role of Noneconomic Criteria', Journal of Marketing, vol. 60 (4), pp. 71-87.

Du Gay, P. and Salaman, G. (1992), 'The Cult(ure) of the Customer', Journal of Management Studies, vol. 29 (5), pp. 615-633.

Dyer, W.G. and Wilkins, A.L. (1991), 'Better Stories, Not Better Constructs to 
Generate Better Theory: A Rejoinder to Eisenhardt', Academy of Management Review, vol. 16 (3), pp. 613-619.

Easterby-Smith, M., Thorpe, R. and Lowe, A. (1991), Management Research, Sage Publications, London.

Easton, G. (1992), 'Industrial Networks: A Review' in B. Axelsson and G. Easton (eds.), Industrial Networks: A New View of Reality, Routledge, London.

Eisenhardt, K.M. (1989), 'Building Theories from Case Study Research', Academy of Management Review, vol. 14 (4), pp. 532-550.

Eisenhardt, K.M. (1991), 'Better Stories and Better Constructs: The Case for Rigor and Comparative Logic', Academy of Management Review, vol. 16 (3), pp. 620-627.

Elkington, J. and Burke, T. (1989), The Green Capitalists, Victor Gollancz, London.

Elkington, J., Knight, P. and Hailes, J. (1991), The Green Business Guide: How To Take Up - And Profit From - The Environmental Challenge, Victor Gollancz, London.

Epstein, E.M. (1987), 'The Corporate Social Policy Process: Beyond Business Ethics, Corporate Social Responsibility, and Corporate Social Responsiveness', California Management Review, vol. 29 (3), pp. 99114.

Evan, W.M. and Freeman, R.E. (1988), 'A Stakeholder Theory of the Modern Corporation: Kantian Capitalism', in T.L. Beauchamp and N.E. Bowie (eds.), Ethical Theory and Business (3/e), Prentice-Hall, Englewood Cliffs.

Evered, R and Louis, M.R. (1981), 'Alternative Perspectives in the Organizational Sciences', Academy of Management Review, vol. 6 (3), pp. 385-395.

Featherstone, M. (1991), Consumer Culture and Postmodernism, Sage, London.

Ferrell, O.C. and Fraedrich, J. (1989), 'A Synthesis of Ethical Decision Models for Marketing', Journal of Macromarketing, vol. 9 (Fall), pp. 55-64.

Ferrell, O.C. and Weaver, K.M. (1978), 'Ethical Beliefs of Marketing 
Managers', Journal of Marketing, vol. 42 (4), pp. 69-73.

Fineman, S. (1995), 'Moral Meanings and Green Organizational Change', paper presented at the Academy of Management Meeting, Vancouver.

Fineman, S. (1996), 'Emotional Subtexts in Corporate Greening', Organization Studies, vol. 17 (3), pp. 479-500.

Fineman, S. (1997), 'Constructing the Green Manager', British Journal of Management, vol. 8 (1), pp. 31-38.

Fineman, S. and Clarke, K. (1996), 'Green Stakeholders: Industry Interpretations and Response', Journal of Management Studies, vol. 33 (6), pp. 715-730.

Fineman, S. and Mangham, I. (1983), 'Data, Meanings and Creativity: A Preface', Journal of Management Studies, vol. 20 (3), pp. 295-300.

Fisk, G. (1973), 'Criteria for a Theory of Responsible Consumption', Journal of Marketing, vol. 37 (2), April, pp. 24-31.

Ford, D. (1980), 'The Development of Buyer-Seller Relationships in Industrial Markets' European Journal of Marketing, vol. 14 (5/6), pp. 339-353.

Ford, D. (1997), Understanding Business Markets (2/e), Dryden Press, London.

Ford, R. and Mclaughlin, F. (1984), 'Perceptions of Socially Responsible Activities and Attitudes: A Comparison of Business School Deans and Corporate Chief Executives', Academy of Management Journal, vol. 27 (3), pp. 660-674.

Fox, K.A. and Kotler, P. (1980), 'The Marketing of Social Causes: The First Ten Years', Journal of Marketing, vol. 44 (4), fall, pp. 24-33.

Frances, J. and Garnsey, E. (1996), 'Supermarkets and Suppliers in the United Kingdom: System Integration, Information and Control', Accounting, Organizations and Society, vol. 21(6), pp. 591-610.

Frederick, W.C., Post, J.E. and Davis, K. (1992), Business and Society: Corporate Strategy, Public Policy, Ethics, (7/e International), McGrawHill, Singapore.

Freeman, R.E. (1984), Strategic Management: A Stakeholder Approach, Pitman, 
Boston.

Freeman, R.E. and Gilbert, D.R. (1988), Corporate Strategy and the Search for Ethics, Prentice-Hall, Englewood Cliffs.

Friedman, M. (1988), 'The Social Responsibility of Business Is to Increase Its Profits', in T.L. Beauchamp and N.E. Bowie, (eds.), Ethical Theory and Business (3/e), Prentice-Hall, Englewood Cliffs.

Galbraith, J.K. (1977), The Affluent Society (3/e rev.), Penguin, Harmondsworth.

Galbraith, J.K. (1972), The New Industrial State (2/e), Penguin, Harmondsworth.

Gaski, F. (1985), 'Dangerous Territory: The Societal Marketing Concept Revisited', Business Horizons, vol. 28, pp. 42-7.

Geertz, C. (1973), The Interpretation of Cultures, Basic Books, New York.

Gilly, M.C. and Wolfinbarger, M. (1998), 'Advertising's Internal Audience', Journal of Marketing, vol. 62 (1), January, pp. 69-88.

Gladwin, T.N. (1993), 'The Meaning of Greening: A Plea for Organizational Theory' in K. Fischer and J. Schot (eds.), Environmental Strategies for Industry, Island Press, Washington.

Glaser, B.G. and Strauss, A.L. (1967), The Discovery of Grounded Theory, Aldine, New York.

Gorz, A. (1980), Ecology as Politics, trans. P. Vigderman and J. Cloud, Pluto, London.

Gorz, A. (1989), Critique of Economic Reason, trans. G. Handyside and C. Turner, Verso, London.

Gray, R.H. (1992), 'Accounting and Environmentalism: An Exploration of the Challenge of Gently Accounting for Accountability, Transparency and Sustainability', Accounting, Organizations and Society, vol. 17 (5), pp. 399-426.

Gray, R.H., Bebbington, K.J. and Waters, D. (1993), Accounting for the Environment: The Greening of Accountancy Part II, Paul Chapman, London. 
Gray, R.H., Kouhy, R. and Lavers, S. (1995a), 'Corporate Social and Environmental Reporting: A Review of the Literature and a Longitudinal Study of UK Disclosure', Accounting, Auditing and Accountability Journal, vol. 8 (2), pp. 46-77.

Gray, R.H., Kouhy, R. and Lavers, S. (1995b), 'Methodological Themes: Constructing a Research Database of Social and Environmental Reporting by UK Companies', Accounting, Auditing and Accountability Journal, vol. 8 (2), pp. 78-101.

Greening, D.W. and Gray, B. (1994), 'Testing a Model of Organizational Response to Social and Political Issues', Academy of Management Journal, vol. 37 (3), pp. 467-498.

Greenley, G.E. and Foxall, G.R. (1997), 'Multiple Stakeholder Orientation in UK Companies and the Implications for Company Performance', Journal of Management Studies, vol. 34 (2), pp. 259-284.

Greyser, S.A. (1972), 'Advertising: Attacks and Counters', Harvard Business Review, vol. 50 (2), Mar-Apr, pp. 1-9.

Gummesson, E. (1987), 'The New Marketing - Developing Long-Term Interactive Relationships', Long Range Planning, vol. 20 (4), pp. 10-20.

Hakansson, H. and Snehota, I. (1997a), 'No Business is an Island: The Network Concept of Business Strategy' in D. Ford (ed.), Understanding Business Markets (2/e), Dryden Press, London.

Hakansson, H. and Snehota, I. (1997b), 'Analysing Business Relationships', in D. Ford (ed.), Understanding Business Markets (2/e), Dryden Press, London.

Hamel, G., Doz, Y.L. and Prahalad, C.K. (1989), 'Collaborate With Your Competitors - and Win', Harvard Business Review, Jan-Feb, pp. 133139.

Hamel, G. and Prahalad, C.K. (1991), 'Corporate Imagination and Expeditionary Marketing', Harvard Business Review, Jul-Aug, pp. 81-92.

Hamel, G. and Prahalad, C.K. (1994), 'Seeing the Future First', Fortune, 5/9/94, pp. 64-68.

Harte, G., Lewis, L. and Owen, D. (1991), 'Ethical Investment and the Corporate Reporting Function', Critical Perspectives on Accounting, 
vol. 2 (3), pp. 227-253.

Hartman, C.L. and Stafford, E.R. (1997), 'Green Alliances: Building New Business with Environmental Groups', Long Range Planning, vol. 30 (2), pp. 184-196.

Harvey, B. (1994), Business Ethics: A European Approach, Prentice Hall, Hemel Hempstead.

Harvey, B., Smith, S. and Wilkinson, B. (1984), Managers and Corporate Social Policy, Macmillan Press, London.

Hassard, J. (1993) Sociology and Organization Theory, Cambridge University Press, Cambridge.

Heilbroner, R.L., et al. (1972), In the Name of Profit, Doubleday, New York.

Hoffman, A.J. (1993), 'The Importance of Fit Between Individual Values and Organizational Culture in the Greening of Industry', Business Strategy and the Environment, vol. 2 (4), pp. 10-18.

Hoffman, W.M. and Moore, J.M. (1990), Business Ethics: Readings and Cases in Corporate Morality (2/e), McGraw-Hill, New York.

Houston, F.S. (1986), 'The Marketing Concept: What It Is and What It Is Not', Journal of Marketing, vol. 50 (2), April, pp. 81-87.

Hunt, S.D., Kieker, P.L. and Chonko, L.B. (1990), 'Social Responsibility and Personal Success: A Research Note', Journal of the Academy of Management Science, vol. 18 (3), pp. 239-244.

Hunt, S.D. and Vitell, S.J. (1986), 'A General Theory of Marketing Ethics', Journal of Macromarketing, vol. 6, spring, pp. 5-16.

Hunt, S.D. and Vitell, S.J. (1993), 'The General Theory of Marketing Ethics: A Retrospective and Revision', in N.C. Smith and J.A. Quelch (eds.), Ethics in Marketing, Irwin, Homewood.

Hunt, S.D., Wood, V.R. and Chonko, L.B. (1989), 'Corporate Ethical Values and Organizational Commitment in Marketing', Journal of Marketing, vol. 53 (3), July, pp. 79-90.

Hutchinson, C. (1992), 'Corporate Strategy and the Environment', Long Range Planning, vol. 25 (4), pp. 9-21. 
Irvine, S. and Ponton, A. (1988), A Green Manifesto, Macdonald Optima, London.

Iyer, E., Banerjee, B. and Gulas, C. (1994), 'An Exposé on Green Television Ads', Advances in Consumer Research, vol. 21, Association for Consumer Research, Chicago.

Jackall, R. (1988), Moral Mazes, Oxford University Press, New York.

Jarillo, J.C. and Stevenson, H.H. (1991), 'Co-operative Strategies - the Payoffs and the Pitfalls', Long Range Planning, vol. 24 (1), pp. 64-70.

Jick, T.D. (1979), 'Mixing Qualitative and Quantitative Methods: Triangulation in Action', Administrative Science Quarterly, vol. 24, December, pp. 602-611.

Johnson, G. and Scholes, K. (1997), Exploring Corporate Strategy: Text and Cases (4/e), Prentice Hall, Hemel Hempstead.

Jones, T.M. (1991), 'Ethical Decision Making by Individuals in Organizations: An Issue-Contingent Model', Academy of Management Review, vol. 16 (2), April, pp. 366-395.

Kangun, N. (1972), Society and Marketing, Harper and Row, New York.

Kangun, N., Carlson, L. and Grove, S.J. (1991), 'Environmental Advertising Claims: A Preliminary Investigation', Journal of Public Policy and Marketing, vol. 10 (2), pp. 47-58.

Kangun, N. and Polonsky, M.J. (1995), 'Regulation of Environmental Marketing Claims: A Comparative Perspective', International Journal of Advertising, vol. 14 (1), pp. 1-24.

Kassarjian, H. (1971), 'Incorporating Ecology Into Marketing Strategy: The Case of Air Pollution', Journal of Marketing, vol. 35 (3), July, pp. 61-65.

Kellner, D. (1989), Jean Baudrillard: From Marxism to Postmodernism and Beyond, Polity Press, Cambridge.

Kilbourne, W. (1995), 'Green Advertising: Salvation or Oxymoron?', Journal of Advertising, vol. 24 (2), pp. 7-19.

Kilbourne, W., McDonagh, P. and Prothero, A. (1997), 'Sustainable Consumption and the Quality of Life: A Macromarketing Challenge to 
the Dominant Social Paradigm', Journal of Macromarketing, Spring, pp. 4-24.

Kinnear, T.C., Taylor, J.R. and Ahmed, S.A. (1974), 'Ecologically Concerned Consumers: Who Are They?', Journal of Marketing, vol. 38 (2), April, pp. 20-24.

Knights, D. and Morgan, G. (1991), 'Corporate Strategy, Organizations, and Subjectivity: A Critique’, Organization Studies, vol. 12 (2), pp. 251-273.

Knights, D., Sturdy, A. and Morgan, G. (1994), 'The Consumer Rules? An Examination of the Rhetoric and 'Reality' of Marketing in Financial Services', European Journal of Marketing, vol. 28 (3), pp. 42-54.

Kohlberg, L. (1969), 'Stage and Sequence: The Cognitive Development Approach to Socialization' in D.A. Goslin (ed.), Handbook of Socialization Theory and Research, Rand McNally, Chicago.

Kotha, S. (1995), 'Mass Customization: Implementing the Emerging Paradigm for Competitive Advantage', Strategic Management Journal, vol. 16 (special issue), pp. 21-42.

Kotler, P. (1972), 'What Consumerism Means for Marketers', Harvard Business Review, vol. 50, May-June, pp. 48-57.

Kotler, P., Armstrong, G., Saunders, J. and Wong, V. (1996), Principles of Marketing, (European ed.), Prentice-Hall, Hemel Hempstead.

Kotler, P. and Levy, S.J. (1969), 'Broadening the Concept of Marketing', Journal of Marketing, vol. 33 (1), January, pp. 10-15.

Kotler, P. and Roberto, E.L. (1989), Social Marketing: Strategies for Changing Public Behaviour, Free Press, New York.

Kotler, P. and Zaltman, G. (1971), 'Social Marketing: An Approach to Planned Social Change', Journal of Marketing, vol. 35 (3) July, pp. 312.

Krugman, D.M. and Ferrell, O.C. (1981), 'The Organizational Ethics of Advertising: Corporate and Agency Views', Journal of Advertising, vol. $10(1)$, pp. 21-30.

Kuhn, T.S. (1970), The Structure of Scientific Revolution (2/e), University of Chicago Press, Chicago. 
Laczniak, G.R., Lusch, R.F. and Murphy, P.E. (1979), 'Social Marketing: Its Ethical Dimensions', Journal of Marketing, vol. 43 (2), spring, pp. 2936.

Laczniak, G.R. and Murphy, P.E. (1993), Ethical Marketing Decisions: The Higher Road, Allyn and Bacon, Boston.

Lager, F. (1994), Ben and Jerry's: The Inside Scoop, Crown Publishers Inc, New York.

Lamming, R. and Hampson, J. (1996), 'The Environment as a Supply Chain Management Issue', British Journal of Management, vol. 7 (special issue), pp. S45-S62.

Lazer, W. and Kelley, E.J. (1973), Social Marketing: Perspectives and Viewpoints, Richard D. Irwin, Homewood, Illinois.

Lee, J. (1997), 'Is Co-op Ethos Out of Date?', Marketing, 1/5/1997, p.19.

Levitt, T. (1972), 'The Morality(?) of Advertising', in N. Kangun (ed.), Society and Marketing, Harper and Row, New York.

Levitt, T. (1980), 'Marketing Success Through Differentiation - Of Anything', Harvard Business Review, Jan-Feb, pp. 83-91.

Levy, S.J. and Zaltman, G. (1975), Marketing, Society, and Conflict, PrenticeHall, Englewood Cliffs.

Lill, D., Gross, C. and Peterson, R. (1986), 'The Inclusion of Social Responsibility Themes by Magazine Advertisers: A Longitudinal Study', Journal of Advertising, vol. 15 (2), pp. 35-41.

MacIntyre, A. (1984), After Virtue (2/e), University of Notre Dame Press, Notre Dame.

Macnaghten, P. and Urry, J. (1995), 'Towards a Sociology of Nature', Sociology, vol. 29 (2), pp. 203-220.

Marketing Week (1991), 'Boots Introduces Male Natural Collection Range', Marketing Week, 13/9/91, p.7.

Martin, J. (1985), 'Can Organizational Culture Be Managed?', in P.J. Frost, L.F. Moore, M.R. Louis, C. Lundberg and J. Martin (eds.), Organizational Culture, Sage Publications, Newbury Park, Ca. 
Martin, J. (1992), Cultures in Organizations: Three Perspectives, Oxford University Press, New York.

Martin, J. and Siehl, C. (1983), 'Organizational Culture and Counterculture: An Uneasy Symbiosis', Organizational Dynamics, vol. 12 (2), pp. 5264.

Martin, J. (1995), 'Ignore Your Customer', Fortune, 1/5/95, pp. 83-86.

Mathews, B.P. and Diamantopoulos, A. (1995), 'An Analysis of Response Bias in Executive's Self-Reports', Journal of Marketing Management, vol. 11 (8), pp. 835-846.

McCloskey, J., Smith, D. and Graves, B. (1993), 'Exploring the Green Sell: Marketing Implications of the Environmental Movement', in D. Smith (ed.), Business and the Environment: Implications of the New Environmentalism, Paul Chapman Ltd., London.

McDonagh, P. (1995), 'Sustainable Communication: Pipe Dream for Green Advertisers or the New Way for Business to Communicate', in M. Bergadaà (ed.), Marketing Today and for the 21st Centrury, Proceedings of the 24th European Marketing Academy Conference, vol. 1, pp. 731751.

McDonagh, P. (1996), 'Relationship Marketing: Death from Natural Causes!', paper presented at the Department of Marketing Research Seminar, University of Stirling, 22/10/96.

McDonagh, P and Prothero, A. (1997), Green Management: A Reader, Dryden Press, London.

McGuire, J.B., Sundgren, A. and Schneeweis, T. (1988), 'Corporate Social Responsibility and Firm Financial Performance', Academy of Management Journal, vol. 31 (4), pp. 854-872.

Mendleson, N and Polonsky, M.J. (1995), 'Using Strategic Alliances to Develop Credible Green Marketing', Journal of Consumer Marketing, vol. 12 (2), pp. 4-18.

Menon, A. and Menon, A. (1997), "Enviropreneurial Marketing Strategy: The Emergence of Corporate Environmentalism as Market Strategy", Journal of Marketing, vol. 61 (1), January, pp. 51-67.

Miles, M.B. and Huberman, A.M. (1994), Qualitative Data Analysis (2/e), 
Sage Publications, Thousand Oaks, Ca.

Mintel (1995), 'Marketing Intelligence Report: Make-up', January, Mintel, London.

Minton, A.P. and Rose, R.L. (1997), 'The Effects of Environmental Concern on Environmentally Friendly Consumer Behaviour: An Exploratory Study', Journal of Business Research, vol. 40, pp. 37-48.

Mintzberg, H. (1979), 'An Emerging Strategy of 'Direct' Research', Administrative Science Quarterly, vol. 24, December, pp. 582-589.

Mintzberg, H. (1983), 'The Case For Corporate Social Responsibility', Journal of Business Strategy, vol. 4 (2), pp. 3-15.

Mintzberg, H. (1989), Mintzberg on Management, Free Press, New York.

Mintzberg, H. (1990), 'The Design School: Reconsidering the Basic Premises of Strategic Management', Strategic Management Journal, vol. 11 (6), pp. 171-195.

Mintzberg, H. (1991), 'Learning 1, Planning 0: Reply to Igor Ansoff', Strategic Management Journal, vol. 12 (6), pp. 463-466.

Morgan, G. (1983a), 'Research as Engagement: A Personal View', in Morgan, G. (ed.), Beyond Method: Strategies for Social Research, Sage Publications, Newbury Park, Ca.

Morgan, G. (1983b), 'Research Strategies: Modes of Engagement', in Morgan, G. (ed.), Beyond Method: Strategies for Social Research, Sage Publications, Newbury Park, Ca.

Morgan, G. and Smircich, L. (1980), 'The Case for Qualitative Research', Academy of Management Review, vol. 5 (4), pp. 491-500.

Morris, C.W. (1949), Signs, Language and Behaviour, Prentice Hall, New York.

Morris, T. and Wood, S. (1991), 'Testing the Survey Method: Continuity and Change in British Industrial Relations', Work, Employment and Society, vol. 5 (2), pp. 259-282.

Murphy, D.F. (1996a), 'DIY-WWF Alliance: Doing it Together for the World's Forests', School for Policy Studies/New Consumer Working 
Paper, University of Bristol.

Murphy, D.F. (1996b), 'In the Company of Partners. Business. NGOs and Sustainable Development: Towards a Global Perspective', in R. Aspinwall and J. Smith (eds.), Environmentalist and Business Partnerships: A Sustainable Model?: A Critical Assessment of the Impact of the WWF UK 1995 Group, White Horse Press, Cambridge.

Murphy, P.E. and Laczniak, G.R. (1981), 'Marketing Ethics: A Review With Implications for Managers, Educators and Researchers', in B.M Enis and K.J. Roering (eds.), Review of Marketing, American Marketing Association, Chicago.

Naess, A. (1989), Ecology, Community and Lifestyle, trans. and rev. D. Rothenberg, Cambridge University Press, Cambridge.

National Consumer Council (1996), Green Claims: A Consumer Investigation into Marketing Claims About the Environment, National Consumer Council, London.

Newton, T.J. and Harte, G. (1997), 'Green Business: Technicist Kitsch?', Journal of Management Studies, vol. 34 (1), pp. 75-98.

Nichols, T. (1969), Ownership, Control and Ideology, George Allen and Unwin Ltd, London.

Nord, W.R. (1985), 'Can Organizational Culture Be Managed? A Synthesis', in P.J. Frost, L.F. Moore, M.R. Louis, C. Lundberg and J. Martin (eds.), Organizational Culture, Sage, Newbury Park, Ca.

Ogbonna, E. (1992), 'Managing Organizational Culture: Fantasy or Reality?', Human Resource Management Journal, vol. 3 (2), pp. 42-54.

O'Shaughnessy, N.J. (1990), 'High Priesthood, Low Priestcraft: The Role of Political Consultants', European Journal of Marketing, vol. 24 (2), pp. 7-23.

Ostergaard, G.N. and Halsey, A.H. (1965), Power in Co-operatives: A Study of the Internal Politics of British Retail Societies, Basil Blackwell, Oxford.

Owen, C.L. and Scherer, R.F. (1993), 'Social Responsibility and Market Share', Review of Business, vol. 15 (1), pp. 11-16. 
Packard, V. (1957), The Hidden Persuaders, Pocket Books (Cardinal Edition), New York.

Parker, M. (1997), 'Business Ethics and Social Theory: Against Ethics', paper presented at the 1997 British Academy of Management Annual Conference, London Business School, September.

Patten, D.M. (1992), 'Intra-Industry Environmental Disclosures in Response to the Alaskan Oil Spill: A Note on Legitimacy Theory', Accounting, Organizations and Society, vol. 17 (5), pp. 471-476.

Pearce, D., Markandya, A. and Barbier, E.B. (1989), Blueprint for a Green Economy, Earthscan, London.

Peattie, K. (1992), Green Marketing, Pitman, London.

Peattie, K. (1995), Environmental Marketing Management, Pitman, London.

Peattie, K. and Charter, M. (1994), 'Green Marketing' in M.J. Baker (ed.), The Marketing Book (3/e), Butterworth-Heinemann, Oxford.

Peattie, K. and Ratnayaka, M. (1992), 'Responding to the Green Movement', Industrial Marketing Management, vol. 21 (2), pp. 103-110.

Peters, T.J. and Waterman, R.H. (1982), In Search of Excellence, Harpers and Row, London.

Pettigrew, A.M. (1973), The Politics of Organizational Decision-Making, Tavistock, London.

Pettigrew, A.M. (1979), 'On Studying Organizational Cultures', Administrative Science Quarterly, vol. 24, December, pp. 570-581.

Pettigrew, A.M. (1985), The Awakening Giant, Blackwell, Oxford.

Pfeffer, J. (1981), Power in Organizations, Pitman, Marshfield, Mass.

Pfeffer, J. and Salancik, G.R. (1978), The External Control of Organizations: A Resource Dependence Perspective, Harper and Row, New York.

Phillips, B.J. (1997), 'In Defence of Advertising: A Social Perspective', Journal of Business Ethics, vol. 16, pp. 109-118.

Phillips, N. (1991), 'The Sociology of Knowledge: Towards an Existential View 
of Business Ethics', Journal of Business Ethics, vol. 10, pp. 787-795.

Phillips, N. and Brown, J.L. (1993), 'Analyzing Communications In and Around Organizations: A Critical Hermeneutic Approach', Academy of Management Journal, vol. 36 (6), pp. 1547-1576.

Pollay, R.W. (1986), 'The Distorted Mirror: Reflections on the Unintended Consequences of Advertising', Journal of Marketing, vol. 50 (2), April, pp. 18-36.

Poole, M.S. and Van de Ven, A.H. (1989), 'Using Paradox to Build Management and Organization Theories', Academy of Management Review, vol. 14 (4), pp. 562-578.

Porritt, J. (1984), Seeing Green: The Politics of Ecology Explained, Blackwell, Oxford.

Posner, B.Z. and Schmidt, W.H. (1984), 'Values and the American Manager: An Update', California Management Review, vol. 26 (3), spring, pp. 202216.

Posner, B.Z. and Schmidt, W.H. (1992), 'Values and the American Manager: An Update Updated', California Management Review, vol. 34 (3), spring, pp. 80-94.

Prothero, A. (1990), 'Green Consumerism and the Societal Marketing Concept: Marketing Strategies for the 1990s', Journal of Marketing Management, vol. 6 (2), pp. 87-103.

Prothero, A. and McDonagh, P. (1992), 'Producing Environmentally Acceptable Cosmetics? The Impact of Environmentalism on the United Kingdom Cosmetics and Toiletries Industry', Journal of Marketing Management, vol. 8 (2), pp. 147-166.

Prothero, A., McDonagh, P. and Peattie, K. (1994), 'Green Marketing Communications - Dressing Windows or Opening Doors?', in Bell, J. et al. (eds.), Marketing: Unity in Diversity, Proceedings of the 1994 Marketing Education Group Annual Conference, University of Ulster, Vol II, pp. 766-776.

Puxty, A.G. (1986), 'Social Accounting as Immanent Legitimation: A Critique of Technist Ideology', Advances in Public Interest Accounting, vol. 1, pp. 95-112. 
Randall, D.M. and Gibson, A.M. (1990), 'Methodology in Business Ethics Research: A Review and Critical Assessment', Journal of Business Ethics, vol. 9 (6), pp. 457-471.

Ritzer, G. (1997), 'Introduction' in J. Baudrillard, The Consumer Society: Myths and Structures, (English Trans.), Sage, London.

Roberts, J.A. and Bacon, D.R. (1997), 'Exploring the Subtle Relationships between Environmental Concern and Ecologically Conscious Consumer Behaviour', Journal of Business Research, vol. 40, pp. 79-89.

Robin, D.P. and Reidenbach, R.E. (1987), 'Social Responsibility, Ethics and Marketing Strategy: Closing the Gap Between Concept and Application', Journal of Marketing, vol. 51 (1), January, pp. 44-58.

Robin, D.P. and Reidenbach, R.E. (1993), 'Searching for a Place to Stand: Toward a Workable Ethical Philosophy for Marketing', Journal of Public Policy and Marketing, vol. 12 (1), spring, pp. 97-105.

Roddick, A. (1992), Body and Soul, Vermillion, London.

Rowlinson, M. and Hassard, J. (1993), 'The Invention of Corporate Culture: A History of the Histories of Cadbury', Human Relations, vol. 46 (3), pp. 299-326.

Sagoff, M.(1986), 'At the Shrine of Our Lady of Fatima, or Why Political Questions Are Not All Economic', in D. VanDeVeer and C. Pierce (eds.), People, Penguins, And Plastic Trees: Basic Issues in Environmental Ethics, Wadsworth, Belmont.

Salancik, G.R. (1979), 'Field Stimulations for Organizational Behaviour Research', Administrative Science Quarterly, vol. 24, December, pp. 638-649.

Salancik, G.R. and Pfeffer, J. (1977), 'Who Gets Power - And How They Hold On To It: A Strategic Contingency Model of Power', Organization Dynamics, vol. 5, pp. 3-21.

Schein, E.H. (1992), Organizational Culture and Leadership (2/e), Jossey-Bass, San Francisco.

Schlegelmilch, B.B. (1988), 'Targeting of Fund-raising Appeals - How to Identify Donors', European Journal of Marketing, vol. 22 (1), pp. 31-40. 
Schlegelmilch, B.B., Bohlen, G. and Diamantopoulos, A. (1996), 'The Link Between Green Purchasing Decisions and Measures of Environmental Consciousness', European Journal of Marketing, vol. 30 (5), pp. 35-55.

Schlegelmilch, B.B. and Houston, J.E. (1989), 'Corporate Codes of Ethics in Large UK Companies: An Empirical Investigation of Use, Content and Attitudes', European Journal of Marketing, vol. 23 (6), pp. 7-24.

Schot, J. (1992), 'Credibility and Markets as Greening Forces for the Chemical Industry', Business Strategy and the Environment, vol. 1 (1), pp. 35-44.

Schot, J. and Fischer, K. (1993), 'The Greening of the Industrial Firm', in K. Fischer and J. Schot (eds.), Environmental Strategies for Industry, Island Press, Washington D.C.

Schrum, L.J., McCarty, J.A. and Lowrey, T.M. (1995), 'Buyer Characteristics of the Green Consumer and their Implications for Advertising Strategy', Journal of Advertising, vol. 24 (2), pp. 71-82.

Schumacher, E.F. (1974), Small is Beautiful, Abacus, London.

Sethi, S.P. (1974), Unstable Ground: Corporate Social Policy in a Dynamic Society, Melville Publishing, Los Angeles.

Sethi, S.P. (1979), 'A Conceptual Framework for Environmental Analysis of Social Issues and Evaluation of Business Response Patterns', Academy of Management Review, vol. 4 (1), pp. 63-74.

Sharp Paine, L. (1993), 'Children as Consumers: The Ethics of Children's Television Advertising', in N.C. Smith and J.A. Quelch (eds.), Ethics in Marketing, Irwin, Homewood.

Sharp Paine, L. (1994), 'Managing for Organizational Integrity', Harvard Business Review, Mar-Apr, pp. 106-117.

Shrivastava, P. (1993), 'The Greening of Business', in D. Smith (ed.), Business and the Environment: Implications of the New Environmentalism, Paul Chapman Ltd., London.

Shrivastava, P. (1994), 'CASTRATED Environment: GREENING Organizational Studies', Organizational Studies, vol. 15 (5), pp. 705726.

Shrivastava, P. (1995a), 'Ecocentric Management for a Risk Society', 
Academy of Management Review, vol. 20 (1), pp. 118-137.

Shrivastava, P. (1995b), 'Environmental Technologies and Competitive Advantage', Strategic Management Journal, vol. 16 (special issue), summer, pp. 183-200.

Siehl, C. (1985), 'After the Founder: An Opportunity to Manage Culture', in P.J. Frost, L.F. Moore, M.R. Louis, C. Lundberg and J. Martin (eds.), Organizational Culture, Sage, Newbury Park, Ca.

Simintiras, A.C., Schlegelmilch, B.B. and Diamantopoulos, A. (1997), 'Greening the Marketing Mix: A Review of the Literature and an Agenda for Future Research', in P. McDonagh and A. Prothero (eds.) Green Management: A Reader, Dryden Press, London.

Simmons, P. and Wynne, B. (1993), 'Responsible Care: Trust, Credibility, and Environmental Management', in K. Fischer and J. Schot (eds.), Environmental Strategies for Industry, Island Press, Washington.

Simms, C. (1992), 'Green Issues and Strategic Management in the Grocery Retail Sector', International Journal of Retail and Distribution Management, vol. 20 (1), pp. 32-42.

Simonian, H. (1995), 'Pitfalls of Eco-Shopping', Financial Times, 5/1/95, p.14.

Sinclair, A. (1993), 'Approaches to Organizational Culture and Ethics', Journal of Business Ethics, vol. 12, pp. 63-73.

Smart, B. (1992), Beyond Compliance: A New Industry View of the Environment, World Resources Institute, Washington D.C.

Smircich, L. (1983a), 'Concepts of Culture and Organizational Analysis', Administrative Science Quarterly, vol. 28, September, pp. 339-358.

Smircich, L. (1983b), 'Studying Organizations as Cultures', in Morgan, G. (ed.), Beyond Method: Strategies for Social Research, Sage Publications, Newbury Park, Ca.

Smircich, L. (1985), 'Is the Concept of Culture a Paradigm for Understanding Organizations and Ourselves?', in P.J. Frost, L.F. Moore, M.R. Louis, C. Lundberg and J. Martin (eds.), Organizational Culture, Sage Publications, Newbury Park, Ca.

Smith, D. (1993a), 'The Frankenstein Syndrome: Corporate Responsibility and 
the Environment' in D. Smith (ed.), Business and the Environment: Implications of the New Environmentalism, Paul Chapman Ltd., London.

Smith, D. (1993b), Business and the Environment: Implications of the New Environmentalism, Paul Chapman Ltd., London.

Smith, N.C. (1990), Morality and the Market: Consumer Pressure for Corporate Accountability, Routledge, London.

Smith, N.C. (1991), 'The Case Study: A Vital Yet Misunderstood Research Method for Management', in N.C. Smith and P. Dainty (eds.), The Management Research Handbook, Routledge, London.

Smith, N.C. (1995), 'Marketing Ethics for the Ethics Era', Sloan Management Review, vol. 36 (4), pp. 85-97.

Smith, N.C. and Quelch, J.A. (1993), Ethics in Marketing, Irwin, Homewood.

Starkey, K. (1990), 'Studies on Transitions: Meanings and Method', Journal of Management Studies, vol. 27 (1), pp. 97-110.

Starkey, K. (1998), 'Durkheim and the Limits of Corporate Culture: Whose Culture? Which Durkheim?', Journal of Management Studies, vol. 35 (2), pp. 125-136

Stead, W.E. and Stead, J.G. (1992), Management for a Small Planet: Strategic Decision Making and the Environment, Sage, Newbury Park.

Steiner, G.A. (1972), 'Social Policies for Business', California Management Review, Winter, pp. 17-24.

Stephenson, T.E. (1963), Management in Co-operative Societies, Heineman, London.

Strauss, A. and Corbin, J. (1990), Basics of Qualitative Research: Grounded Theory Procedures and Techniques, Sage Publications, Newbury Park, Ca.

Stroup, M.A. and Neubert, R.L. (1987), 'The Evolution of Social Responsibility', Business Horizons, Mar-Apr, pp. 22-24.

Swanson, D.L. (1995), 'Addressing a Theoretical Problem by Reorienting the Corporate Social Performance Model', Academy of Management Review, vol. 20 (1), pp. 43-64. 
Taylor, G. and Welford, R. (1994), 'Environmental Strategies of Leading UK Supermarket Chains', in R. Welford (ed.), Cases in Environmental Management and Business Strategy, Pitman, London.

ten Bos, R. (1997), 'Business Ethics and Bauman Ethics', Organization Studies, vol. 18 (6), pp. 997-1014.

Thornhill, J. (1992), 'Balancing Profit and Principle', Financial Times, 22/6/92, p.5.

Tombs, S. (1993), 'The Chemical Industry and Environmental Issues', in D. Smith (ed.), Business and the Environment: Implications of the New Environmentalism, Paul Chapman Ltd., London.

Trevino, L.K. (1986), 'Ethical Decision Making in Organizations: A PersonSituation Interactionist Model', Academy of Management Review, vol. 11 (3), pp. 601-617.

Trevino, L.K. and Nelson, K.A. (1995), Managing Business Ethics: Straight Talk About How To Do It Right, John Wiley, New York.

Tribe, L.H. (1986), 'Ways Not to Think about Plastic Trees: New Foundations for Environmental Law', in D. VanDeVeer and C. Pierce (eds.), People, Penguins, And Plastic Trees: Basic Issues in Environmental Ethics, Wadsworth, Belmont.

Turner, B. (1971), Exploring the Industrial Subculture, Macmillan, London.

Turner, B.A. (1983), 'The Use of Grounded Theory for the Qualitative Analysis of Organizational Behaviour', Journal of Management Studies, vol. 20 (3), pp. 333-348.

Turner, B.A. (1992), 'The Symbolic Understanding of Organizations' in M. Reed and M. Hughes (eds.), Rethinking Organization, Sage, London.

Ullmann, A. (1985), 'Data in Search of a Theory: A Critical Examination of the Relationship Among Social Performance, Social Disclosure, and Economic Performance', Academy of Management Review, vol. 10 (3), pp. 540-577.

Unsworth, B. (1992), Sacred Hunger, Hamish Hamilton, London.

Vandermerwe, S. and Oliff, M.D. (1990), 'Customers Drive Corporations Green', Long Range Planning, vol. 23 (6), pp. 10-16. 
Vander Weyer, M. (1995), 'Good Value at Boots', Management Today, September, pp. 42-47.

Van Maanen, J. (1973), 'Observations on the Making of Policemen', Human Organization, vol. 32 (4), pp. 407-418.

Van Maanen, J. (1979a), 'Reclaiming Qualitative Methods for Organizational Research: A Preface', Administrative Science Quarterly, vol. 24, December, pp. 520-526.

Van Maanen, J. (1979b), 'The Fact of Fiction of Organizational Ethnography', Administrative Science Quarterly, vol. 24, December, pp. 539-550.

Van Maanen, J. (1991), 'The Smile Factory: Work at Disneyland', in P.J. Frost, L.F. Moore, M.R. Louis, C.C. Lundberg and J. Martin (eds.), Reframing Organizational Culture, Sage Publications, Newbury Park, Ca.

Van Maanen, J. and Barley, S.R. (1985), 'Fragments of a Theory', in P.J. Frost, L.F. Moore, M.R. Louis, C. Lundberg and J. Martin (eds.), Organizational Culture, Sage Publications, Newbury Park, Ca.

Varadarajan, P.R. and Rajaratnam, D. (1986), 'Symbiotic Marketing Revisited', Journal of Marketing, vol. 50 (1), January, pp. 7-17.

Vitell, S.J., Rallapalli, K.C. and Singhapakdi, A. (1993), 'Marketing Norms: The Influence of Personal Moral Philosophies and Organizational Ethical Culture', Journal of the Academy of Management Science, vol. 21 (4), pp. 331-337.

Walley, N. and Whitehead, B. (1994), 'Its Not Easy Being Green', Harvard Business Review, May-Jun, pp. 46-52.

Wasik, J.F. (1996), Green Marketing and Management: A Global Perspective, Blackwell, Cambridge, Mass.

Waters, J.A. and Bird, F. (1987), 'The Moral Dimension of Organizational Culture', Journal of Business Ethics, vol. 6, pp. 15-22.

Watson, T.J. (1994a), In Search of Management: Culture, Chaos and Control in Managerial Work, Routledge, London.

Watson, T.J. (1994b), 'Managing, Crafting and Researching: Words, Skill and Imagination in Shaping Management Research', British Journal of Management, vol. 5 (special issue), S77-S87. 
Weick, K.E. (1989), 'Theory Construction as Disciplined Imagination', Academy of Management Review, vol. 14 (4), pp. 516-531.

Weick, K.E. (1995), Sensemaking in Organizations, Sage, Thousand Oaks, Ca.

Welford, R. (1995), Environmental Strategy and Sustainable Development, Routledge, London.

Welford, R. and Gouldson, A. (1993), Environmental Management and Business Strategy, Pitman, London.

Wilkins, A.W. (1983), 'Organizational Stories as Symbols Which Control the Organization', in L.R. Pondy, P.J. Frost, G. Morgan, and T.C. Dandridge (eds.), Organizational Symbolism, JAI Press, Greenwich, Conn.

Williamson, J. (1978), Decoding Advertisements, Marion Boyars, London.

Williamson, J. (1986), Consuming Passions, Marion Boyars, London.

Wong, V., Turner, W. and Stoneman, P. (1996), 'Marketing Strategies and Market Prospects for Environmentally-Friendly Consumer Products', British Journal of Management, vol. 7 (3), pp. 263-281.

Wood, D.J. (1991), 'Corporate Social Performance Revisited', Academy of Management Review, vol. 16 (4), pp. 691-718.

Wood, V.R., Chonko, L.B. and Hunt, S.D. (1986), 'Social Responsibility and Personal Success: Are They Incompatible?', Journal of Business Research, vol. 14, pp. 193-212.

World Commission on Environment and Development (1987), Our Common Future, Oxford University Press, Oxford.

Yeo, S. (1995), Who Was J.T.W. Mitchell?, CWS Membership Services, Manchester.

Yin, R. (1989), Case Study Research: Design and Methods (Rev/e), Sage Publications, Newbury Park, Ca. 


\section{Appendix A: Sample Contact Letters and Research Proposals}

The following pages contain examples of some of the contact letters and research proposals used to secure access, as well as some of the basic interview schedules used to collect data. A range of different documents were prepared for this purpose, according to the characteristics of the targeted firm, as well as the stage of data collection that had been reached. The grounded theory approach adopted here meant that these were constantly revised, and became more specific as the project developed. The samples are presented chronologically according to when they were first written. 


\section{MOTIVATIONS AND METHODS IN GREEN MANAGEMENT AND MARKETING: OUTLINE PROPOSAL}

\section{OBJECTIVES}

The main objectives of this study are to:-

- Determine which opportunities and threats are perceived by company as being significant for their own environmental activities and for the environment generally.

- Understand the processes through which management and marketing strategies have been greened (and the barriers which have prevented them from being so).

- Discover the elements of organisational culture which contribute substantially to the success or failure of greener management strategies and marketing campaigns.

- Ascertain the way in which environmental issues are addressed and communicated by and within the company.

- Understand the effects of green initiatives on the experience of managers and employees in the company.

\section{BACKGROUND}

This project shall form the core of my ongoing $\mathrm{PhD}$ research which I am conducting in the School of Management and Finance at the University of Nottingham. Having begun the preliminary work for the project inspring 1994, my research in several companies this year will constitute the second and most important stage in the development of my thesis. I am attempting here to provide a true picture of how environmental initiatives are developed and operationalised - and the effect this has on the company's culture and on its managers and employees. I would like to emphasise that the main purpose of this work is to analyse and to interpret the experience of companies involved in environmental management and marketing and not to evaluate or to criticise them.

The third and final stage of the project, that of writing up and compiling this information into a final thesis, I am expecting to complete sometime in summer 1997. My ultimate aim then is to develop a theoretical framework to explain how businesses can effectively and practically meet the long run social and environmental challenge and the effect this has on the company's culture. 


\section{EXPECTED FORMAT OF PROJECT}

Given that a great deal rests on the decisions of those companies involved in the project, the exact format has not been decided in advance. Ideally however my contact with the companies would:-

- Be conducted at one or more sites in the company.

- Take place on a number of occasions, perhaps over several months.

- Involve several one hour interviews with staff at different levels in the organisation. Although most interviewees would only be expected to be interviewed once, some re-interviews may be necessary.

- Include some access to relevant documents, minutes of meetings, internal and external reports etc.

- Entail some observation of routine activities and meetings involving the environmental function.

\section{OUTCOMES}

- Full anonymity of individuals and of the company can be guaranteed when and where it is required.

- The company shall also be offered a copy of the final thesis, or a summary report, if it so wishes. It is hoped that this will prove useful in:-

- The development and implementation of present and future environmental management strategies.

- Gaining a fuller understanding of the relationship between the company's culture and its environmental management practises and processes. 


\section{Main Objective}

To get a clearer picture of the environmental concerns which impact upon the company/consumer interface (i.e. at the store).

\section{Main Themes}

Principal areas in which to focus + main questions to be explored:

\section{Issues}

- How important is the environment to the general running of the store?

- Which environmental issues do stores have to contend with on a day to day level?

- Which are given most priority?

-Who takes responsibility for these?

- Where does the motivation come from to initiate environmental activities in-store?

- What processes are in place to meet environmental demands?

\section{Staff}

- How much do environmental concerns impact upon store staff's roles/positions?

- How involved are staff in environmental activities?

- How interested are they? Who is most interested? To what extent is this jobrelated?

- How knowledgeable are store staff about the company's environmental activities?

- What impact does company training have?

\section{Consumers}

- How concerned are consumers about environmental issues at the point of purchase? - Which of the company's environmental initiatives do they seem most interested in?

- Do they seek information/advice? Do they complain?

- How are these demands handled?

- How have these concerns changed over time?

\section{Communications}

- In which ways are the company's environmental responses communicated to consumers at the point of purchase?

- Which ways are most successful?

- How could this be improved (if at all)?

- Which staff or product groups tend to be most involved in environmental communications?

- How aware are staff of environmental communications from other corporate divisions? 


\section{Local Community}

- How involved are stores in environmental initiatives of local communities?

-What role do they play?

- How are issues regarding the store and the environment handled with the local authority/local businesses etc? 
The main areas to be covered are:

\section{Management}

' What is the company's strategy regarding the environment? Which individuals/departments are responsible for its implementation?

' Which processes/mechanisms are used to support the integration of environmental considerations into business practice (and which tend to prevent them from being so)?

1 How does organisational culture contribute to the success or failure of environmental initiatives?

' How do different sections/functions/individuals perceive environmental concerns in the business?

\section{Motives}

- What are the main motivations behind moves to include environmental considerations in the activities of the company?

- Which opportunities and threats are perceived as being most important in driving the company's environmental agenda? How has this changed over time?

Why do particular environmentally relevant initiatives get implemented rather than others? How is this decision reached and by whom?

\section{Communication}

' In which ways are marketing and communication strategies oriented towards environmental issues? Which are most successful?

How concerned are consumers about the company's environmental performance? How is this tracked?

How are different audiences identified, prioritised and targeted?

How important is internal communication to the integration of environmental considerations? 


\section{Employees}

- How is the experience of managers and employees in the company affected by these changes?

' How involved/interested/knowledgeable are staff about environmental concerns?

' What impact does company training and other communications have on these responses?

\section{Other Organisations}

What role do other related commercial organisations (suppliers/competitors/retailers etc) have?

How involved does the company become with Government bodies, NGOs, and other pressure groups?

' How are these relationships managed? 


\section{Motivations}

- How/when/who did the initial impetus for the WWF 95 project first come about?

- What were the main motivations for becoming involved in the project?

- Have any of your competitors become involved? When? Why do you think they have/haven't?

- Why has this particular initiative been implemented rather than others? How was this decision reached and by whom? How difficult was it to persuade top management/others in the company?

\section{Management}

- Have any changes in the company been necessary to accommodate this project?

- How do you go about assessing the environmental performance of your company and your suppliers? Which processes/mechanisms are used? How certain can you be?

- Who is responsible for the WWF project? How is their performance assessed?

- How important is the management of the WWF project compared to other company projects? How easy/difficult is it to gain acceptance and commitment from other depts/individuals?

- What are the differences/benefits/problems associated with working with the WWF?

\section{Human Resource}

- How did you personally become involved in the project? Why?

- Who else is involved in the WWF project? How and why did they become involved?

- Has the involvement changed you or the way you think about the environment at all? Are you or your colleagues environmentalists? How easy is it to take a particular stance and reconcile it with your work in the project? 


\section{Communication}

- How is the company's involvement in the project communicated to internal and external audiences? Who is in charge of this? Why is it done this way?

- How concerned are consumers about the company's environmental performance? How is this tracked? What marketing advantages do you think the WWF project will offer the company?

- How are different audiences identified, prioritised and targeted?

\section{Policy}

- How broadly do you define the environment generally? Why? Who decides?

- What is the company's general strategy towards the environment? How does the WWF project fit into this?

- What other outside bodies is the company involved with? Why was the WWF adjudged to be a better partner than other pressure groups? 


\section{WWF 95: Interview Schedule for Environmental Consultants}

\section{Motivations}

- How/when/who did the initial impetus for the WWF 95 project first come about?

- Have any of your competitors become involved? When? Why do you think they have/haven't? How do non-members see the group?

- Why has this particular initiative been implemented rather than others? How was this decision reached and by whom? How easy is it to gain acceptance and commitment from others in the firm?

\section{Management}

- Have any changes in the company been necessary to accommodate this project? How much resource is committed/necessary?

- How do you go about assessing the environmental performance of your company and your suppliers? How many questionnaires? How devised? How certain can you be about responses?

- Length of supply chains? Supplier relationships/response rates?

- Who is responsible for the WWF project? How is their/overall performance assessed? How likely is 1999 target?

\section{Actors}

- How did the consultancy become involved in the project? Why do you think it was used?

- What are the differences/benefits/problems associated with working with the WWF/other group members? How does the Plus Group compare to other environmental projects?

- How is power/authority balanced between the company-consultancyWWF? Why? How rigid/permanent is this?

- Has the involvement changed the way you think about the environment at all? Are you or your colleagues environmentalists? How easy is it to take a particular stance and reconcile it with your work in the project? 


\section{Communication}

- How is the company's involvement in the project communicated to internal and external audiences? Who is in charge of this? Why is it done this way?

- How concerned are customers about the company's environmental performance? How is this tracked? What marketing advantages do you think the WWF project will offer the company?

\section{Policy}

- How broadly is the environment defined generally? Why? Who decides?

- What is the company's general strategy towards the environment? How does the WWF project fit into this?

- What other outside bodies is the company involved with? Why was the WWF adjudged to be a better partner than other pressure groups? 
I am writing with regard to the research that I am currently conducting to complete my $\mathrm{PhD}$ in the School of Management and Finance at the University of Nottingham. The purpose of this research is to analyse the ways in which various local and national companies integrate environmental concerns into their management and marketing activities.

I am currently conducting fieldwork for this project. At present I am completing case studies on various companies, such as Boots the Chemist, CWS, Laing Homes, East Midlands Electricity, Beauty Without Cruelty, and the Ecology Buiding Society.

I was hoping that it might also be possible to use « 3 » as a case study in this regard. Depending on your wishes, the possible scale of « 3 »'s involvement might be anything from one or two interviews with key personnel to an in-depth analysis of the company's environmental management and marketing operations.

If you feel that you are able to offer me any kind of assistance in this matter, then please contact me at your convenience here at the address/phone/fax above.

Thank you for your time and consideration. I look forward to hearing from you soon.

Yours sincerely,

\section{Dear « 2»,}

I am writing with regard to the research that I am currently conducting to complete my $\mathrm{PhD}$ in the School of Management and Finance at the University of Nottingham. The purpose of this research is to analyse the ways in which various companies integrate environmental concerns into their management and marketing activities.

I have recently begun to look at the work of the WWF 1995 Plus Group, and I have interviewed representatives of a number of group companies such as Boots the Chemist, BBC Magazines, Laing Homes and WH Smith among others. I was hoping also that it might be possible to interview yourself, and other relevant colleagues, regarding your experiences in this and other environmental projects. Depending on your wishes, the scale of « 3 »'s involvement might be anything from one or two interviews with key personnel to around five interviews with representatives from various relevant sections in order to develop a more in-depth analysis of the company's environmental management and marketing operations.

If you are able to offer me any kind of assistance in this matter, then please contact me at your convenience here at the address/phone/fax above.

Thank you for your time and consideration. I look forward to hearing from you soon. 


\section{Appendix B: 1995 Plus Group Members ${ }^{75}$}

\section{COMPANY}

Acrimo Limited

Akzo Nobel Decorative Coatings UK and Eire

A M Paper Converters Ltd

$\mathrm{B} \& \mathrm{Q}$ plc

BBC Magazines

Bernstein Group plc

Bioregional Charcoal Co. Ltd.

C. Blumsom Ltd

The Body Shop

Boots The Chemists

Borden Decorative Products Ltd

British Woodlands Ltd

Richard Burbidge Ltd

W.M. Chalke \& Sons Limited

Chartan-Aldred

Chindwell Company Limited

Clarks Wood Company Ltd

\section{AREA OF BUSINESS}

Curtain poles and accessories

Manufacturers of paints and wallcoverings

Converters of toilet and facial tissue and kitchen towels

DIY and Garden Centre Superstore

Consumer Magazine Publisher

Manufacturers of fitted furniture

Suppliers of British charcoal and firewood

Importers of hardwood

Cosmetic suppliers

Retail chemists

Manufacturers of wallpaper, vinyl coverings and accessories

Charcoal

Moulded wood products

Makes wooden replacement handles for tools

Manufactures charcoal

Supplier of doors

Timber importers

\footnotetext{
75 These were the named members of the Plus Group as at July 1996. Source: WWF documentation.
} 
Core Products Ltd

Crowley Esmonde Ltd

CWS

Datamatic Limited

David Craig

Do It All Limited

Douglas Kane Hardware

Dudley Stationery Ltd

Ecological Trading Company plc

Fine Decor plc

Forbo-Lancaster Ltd

Future Publishing Ltd

Graham \& Brown Ltd

Great Mills (Retail) Ltd

Harrison Drape

Homebase

Hunt \& Broadhurst Ltd

Indian Ocean Trading Company

Intercraft Products Ltd

Jac by the Stowl

John Dickinson Stationery Ltd
Importers and distributors of pine

shelving and storage units

Stationery

Co-operative retail

Supplier of laser paper

Garden Furniture

DIY retailer

Manufacturer and supplier of hardware, cabinet furniture

Commercial Contract Stationery

Import and processing timber

Manufacturer of wall coverings

Designer, manufacturer and supplier of paper backed wall-coverings

Magazine Publishers

Manufacturer of wall-coverings

DIY retail chain

Assembler and distributor of curtain and shower poles

DIY retailer

Manufacturer of stationery

Outdoor furniture

Manufacturers of picture frames

Handmade British woodland products

Manufacturers of envelopes and stationery products 
Laing Homes Ltd

F H Lee Ltd

Linea Housewares Ltd

Magnet Ltd

F W Mason \& Sons Limited

Milland Fine Timber

Moores of Stalham (UK) Ltd

Mosely-Stone

M\&N Norman

NEXT dimension plc

Niceday Ltd

Parlour Products Ltd

Perstorp Unidur Limited
Larch-Lap Limited

Saw-milling home grown and imported timber for garden products

House builders

Manufacturer of toilet rolls and kitchen towels

Manufacturer of clothes pegs, washing lines and domestic brushware

DIY Retailer

Manufacturers and distributors of solid wood products

Sawmillers and suppliers of hardwoods

Manufacturers of starch tray, wooden housewares etc.

Manufacturers paint brushes, rollers and decorators materials

Importers of hardwoods

Manufacturers of kitchens

Stationery products

Charcoal and $B B Q s$

Manufacturer of printed paper and foils for furniture industry

Premium Timber Products Limited Importing and distributing DIY products

Railtrack PLC

National Railway infrastructure

Rectella International Ltd

Manufacturer of charcoal

Rothley Limited

Manufacturers of decorative tube and fittings

J. Sainsbuy plc

Retailers of diverse range of paper, pulp and timber products

Manufacturers of wood panels, and doors 
Sherwood Promark Limited

Shireclose Housewares Ltd

W.H. Smith Retail Ltd

Spicers Ltd Manufacturing

Spur Shelving

Swish Products Limited

T\&G Woodware Ltd

Tesco plc

True Temper Ltd

Vymura plc

E C Walton \& Co Ltd
Manufacture and distributor of charcoal

Supplier of domestic kitchen woodware

Stationer and Newsagent

Manufacturers of envelopes, notepads and books

Manufacture of shelving systems

Curtain rails and accessories

Suppliers of kitchen woodware

Food retailer

Suppliers of garden tools

Designs, manufactures and distributes wall coverings

Manufacturers and suppliers of garden buildings

Wickes Building Supplies Limited Retailer of timber, building supplies and home improvement products

John Wilman Ltd

Manufacturer of wall coverings

Woodbridge Timber Ltd

Woodlam Products
Distributor of dry, graded and processed timber

Trader in wood products

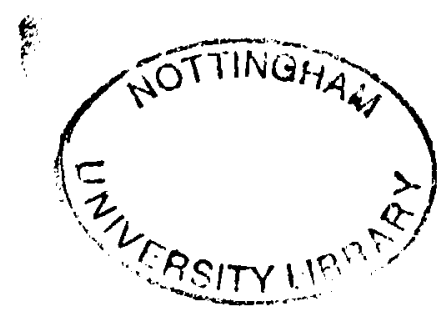

